BNL-113354-2016-TH

\title{
Dynamic Conformations of Nucleosome Arrays in Solution from Small-Angle X-ray Scattering
}

\author{
by Steven C. Howell
}

B.S. in Physics, April 2009, Brigham Young University

M.S. in Physics, May 2013, The George Washington University

A Dissertation submitted to

The Faculty of

The Columbian College of Arts and Sciences

of The George Washington University in partial fulfillment of the requirements

for the degree of Doctor of Philosophy

January 31, 2016

Dissertation directed by

Xiangyun Qiu

Assistant Professor of Physics

Notice: This Thesis/Dissertation describes work supported by Brookhaven Science Associates, LLC under Contract No. DE-SC0012704 with the U.S. Department of Energy. The United States Government retains a non-exclusive, paid-up, irrevocable, world-wide license to publish or reproduce the published form of this manuscript, or allow others to do so, for United States Government purposes. 
ProQuest Number: 3738561

All rights reserved

INFORMATION TO ALL USERS

The quality of this reproduction is dependent upon the quality of the copy submitted.

In the unlikely event that the author did not send a complete manuscript and there are missing pages, these will be noted. Also, if material had to be removed, a note will indicate the deletion.

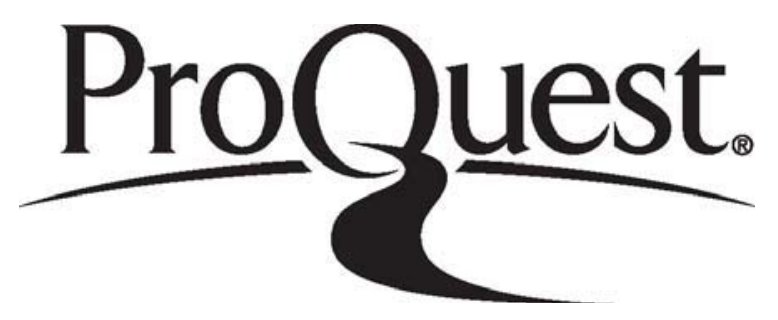

ProQuest 3738561

Published by ProQuest LLC (2015). Copyright of the Dissertation is held by the Author.

All rights reserved.

This work is protected against unauthorized copying under Title 17, United States Code Microform Edition (c) ProQuest LLC.

ProQuest LLC.

789 East Eisenhower Parkway

P.O. Box 1346

Ann Arbor, Ml $48106-1346$ 
The Columbian College of Arts and Sciences of The George Washington University certifies that Steven Craig Howell has passed the Final Examination for degree of Doctor of Philosophy as of November 20, 2015. This is the final and approved form of the dissertation.

\section{Dynamic Conformations of Nucleosome Arrays in Solution from Small-Angle X-ray Scattering}

Steven C. Howell

Dissertation Research Committee:

Xiangyun Qiu, Assistant Professor of Physics, Dissertation Director

Mark Reeves, Professor of Physics, Committee Member

Joseph E. Curtis, Research Chemist, National Institute of Standards and

Technology, Committee Member 
(C) Copyright 2015 by Steven C. Howell All rights reserved 


\section{Dedication}

To my father, who taught me to work hard, my mother, who taught me to never give less than my best, and above all, to my wife, who taught me to live and to love. 


\section{Acknowledgments}

I am indebted to countless family members, mentors, and friends who have supported me throughout my pursuit of this degree. Their influence inspires and motivates me to succeed, while also making that success worthwhile. As I cannot recognize all who have helped me to this point, I will specifically mention those who have played a significant role during my graduate studies.

First and foremost, I would like to thank my family. They have always been supportive throughout my academic and other pursuits. I am especially grateful to my mother and my in-laws for the time their help caring for my boys, who were both born during my graduate studies. To my wife, Rachael, I do not know how to fully express my deepest appreciation for her tremendous encouragement, support, and confidence. My will to persist through challenges originates in the perspective provided by her and our two boys.

I would also like to express my utmost gratitude and respect to my advisor, Xiangyun Qiu. The time I have spent working as part of his group has taught me how to be a successful research scientist. In our interactions, I have experienced unwavering confidence, limitless patience, and above all, his desire that I succeed.

I am also incredibly grateful for the fantastic researchers I have been able to collaborate with; this research would not have been possible without their combined efforts. Specifically, my thanks goes out to Kurt Andresen, for helpful scientific discussions and assistance in performing SAXS experiments. To those from NIH, Yawen Bai and Bing-Rui Zhou, thank you for providing the $4 \times 167 \mathrm{gH} 5$ and $12 \times 167$ nucleosome samples. To those from Purdue University, Chongli Yuan, Isabel Jimenezuseche, and Agnes Mendonca, thank you for providing DNA templates and assisting in preparing histone octamers. To the researchers at GWU, Fuyou Ke, Wei Meng, Carina Lorenzen, Suzanne Arnott, Raju Timsina, and Ben Kopchick, it has been a 
pleasure to work with you. I would also like to recognize Joseph Curtis, the University of Tennessee, and all the scientist at the NIST Center for Neutron Research, for supporting me as a guest researcher and providing the computing resources used for this work.

During my time at GWU, there have been many others who have had a positive influence on me. I would like to thank Harald Grießhammer and Andre Alexandru for pushing me to excel academically as a new graduate student. I owe a great deal to Qi Xia, Kevin Sykora, Marty McHugh, and Trevor Balint for the opportunity to work together in mastering the core physics concepts. I am grateful to Adam Hughes for informative scientific and programming discussions as well as introducing me to several valuable Python resources. I also want to thank Chris Verhaaren for reviewing this dissertation and also for his friendship and helpful scientific discussions.

To the broader community, thank you to those unnamed individuals who have contributed to my success. I appreciate all those from Research Gate and the Stack Exchange communities who provided helpful input in response to my programming, statistics, chemistry, Linux, $\mathrm{H}_{\mathrm{E}} \mathrm{X}$, and other queries. I owe a huge thanks to Wingware Python IDE for the complimentary professional license they provided as this application became an integral part of software development and data analysis. Additionally, I am grateful to the developers of the many open-source software packages that have made my research and programming possible.

I would lastly like to express my appreciation for the funding sources that facilitated my dissertation research, including the facilities and software I used. I am sincerely grateful for the financial support provided through the GWU Academic Excellence Fellowship. This work is based upon research conducted at the Cornell High Energy Synchrotron Source which is supported by the National Science Foundation and the National Institutes of Health/National Institute of General Medical Sciences under NSF award DMR-1332208. Use of the National Synchrotron Light Source, 
Brookhaven National Laboratory, was supported by the U.S. Department of Energy, Office of Science, Office of Basic Energy Sciences, under Contract No. DE-AC0298CH10886. This work benefited from CCP-SAS software developed through a joint EPSRC (EP/K039121/1) and NSF (CHE-1265821) grant. 


\section{Abstract of Dissertation \\ Dynamic Conformations of Nucleosome Arrays in Solution from Small-Angle X-ray Scattering}

Chromatin conformation and dynamics remains unsolved despite the critical role of the chromatin in fundamental genetic functions such as transcription, replication, and repair. At the molecular level, chromatin can be viewed as a linear array of nucleosomes, each consisting of 147 base pairs (bp) of double-stranded DNA (dsDNA) wrapped around a protein core and connected by 10 to $90 \mathrm{bp}$ of linker dsDNA.

Using small-angle X-ray scattering (SAXS), we investigated how the conformations of model nucleosome arrays in solution are modulated by ionic condition as well as the effect of linker histone proteins. To facilitate ensemble modeling of these SAXS measurements, we developed a simulation method that treats coarse-grained DNA as a Markov chain, then explores possible DNA conformations using Metropolis Monte Carlo (MC) sampling. This algorithm extends the functionality of SASSIE, a program used to model intrinsically disordered biological molecules, adding to the previous methods for simulating protein, carbohydrates, and single-stranded DNA. Our SAXS measurements of various nucleosome arrays together with the MC generated models provide valuable solution structure information identifying specific differences from the structure of crystallized arrays. 


\section{Table of Contents}

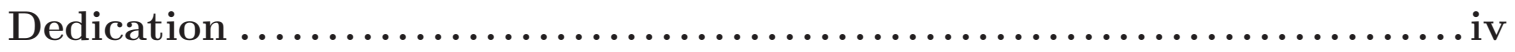

Acknowledgments ...............................................v

Abstract of Dissertation ........................................viii

Table of Contents ...............................................

List of Figures................................................

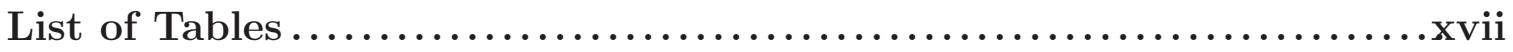

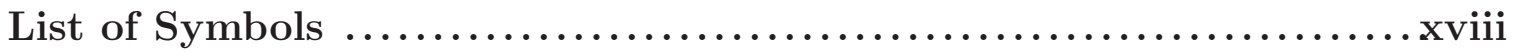

Glossary of Terms and Abbreviations ........................... xxiii

Chapter 1: Introduction and Background $\ldots \ldots \ldots \ldots \ldots \ldots \ldots \ldots \ldots \ldots \ldots \ldots \ldots$

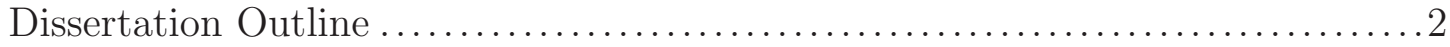

Chromatin: DNA in Eukaryotic Cells ................................... 3

Toward Elucidating Chromatin Structure........................... 4

Factors Affecting Chromatin Structure $\ldots \ldots \ldots \ldots \ldots \ldots \ldots \ldots \ldots \ldots \ldots \ldots$

Experimental Biological Probes ........................................ 9

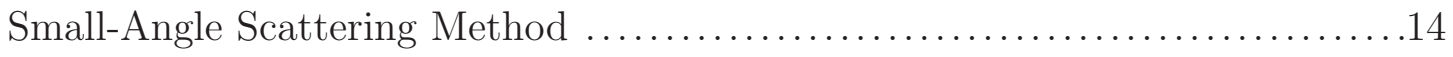

Structure Information From Small-Angle Scattering ..................15

Chapter 2: Structure and Interaction of Nucleosome Monomers .......22

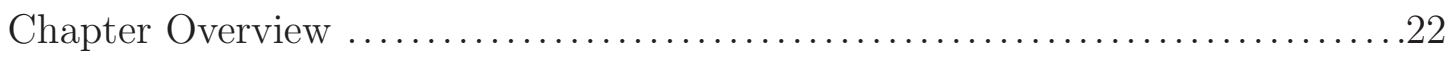

Elucidating Internucleosome Interactions and the Roles of Histone Tails ......22

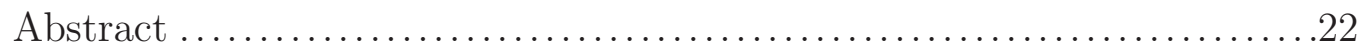

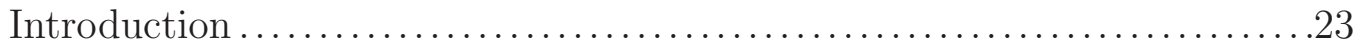




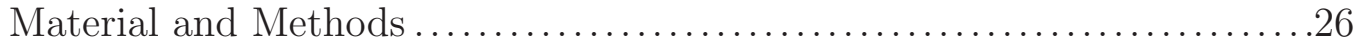

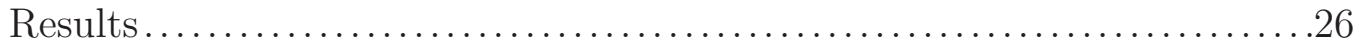

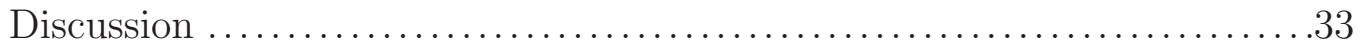

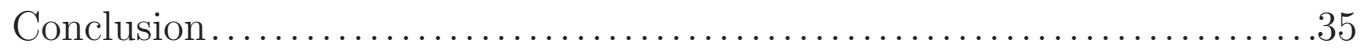

Chapter Summary ................................................ 35

Chapter 3: SAXS Experiments of Nucleosome Arrays .................36

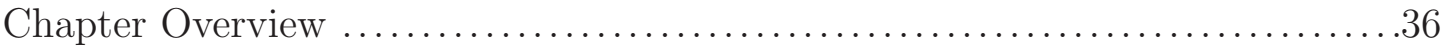

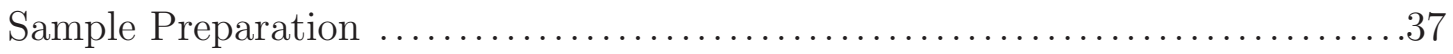

Protein Expression and Purification ..............................37

DNA Template Preparation and Purification .........................38

Octamer Reconstitution and Purification .............................39

Nucleosome Array Reconstitution and Purification ....................40

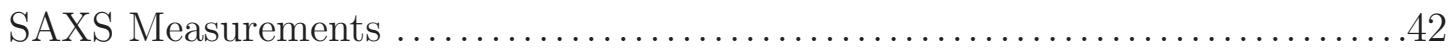

Experimental Configuration and Data Collection ....................42

Data Reduction and Buffer Subtraction ...........................43

Determining the Form Factor ..................................47

Reduced SAXS Profiles.........................................52

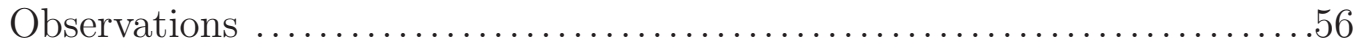

User-Directed Rigid Body Modeling of $4 \times 167$ gH5 Arrays ...................67

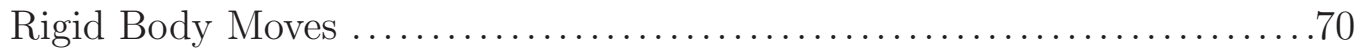

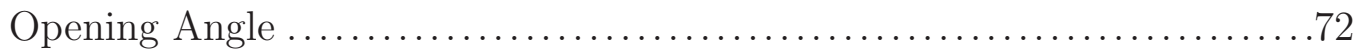

Twist About Dyad Axis .............................................73

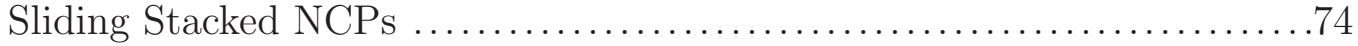

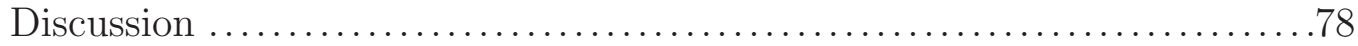

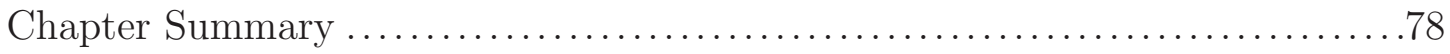

Chapter 4: Double-Stranded DNA Simulation Algorithm .............81 


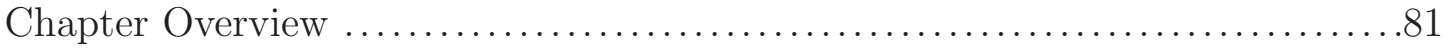

Coarse-Grain Monte Carlo Simulation Algorithm for Double-Stranded DNA...81

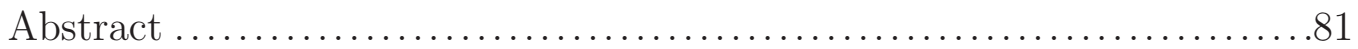

Introduction ............................................... 82

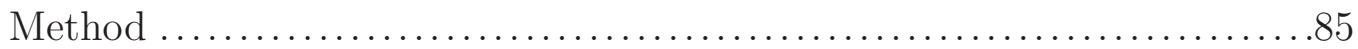

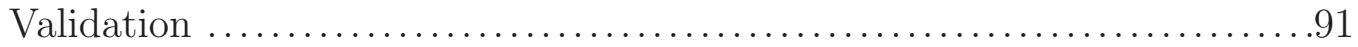

Examples ..................................................... 99

Discussion .................................................. 105

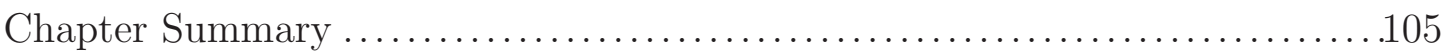

Chapter 5: Nucleosome Array Structure Modeling................107

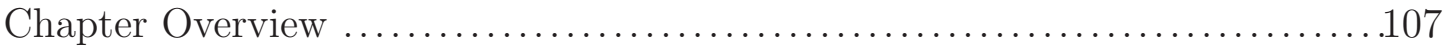

Comparing Discrepancy Metrics.....................................107

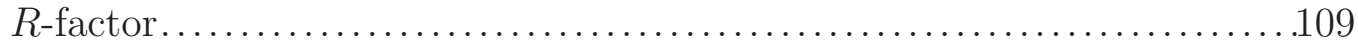

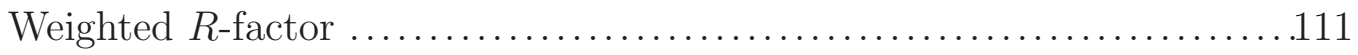

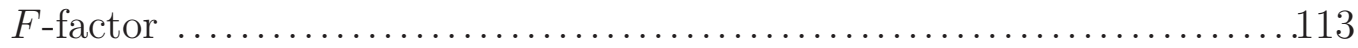

$\chi^{2}$ Statistic ................................................ 114

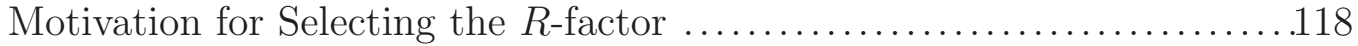

Best Model Results ....................................................119

$4 \times 167$ gH5 Models.............................................. 120

$4 \times 167$ Models ................................................ 122

$3 \times 167$ Models ................................................. 126

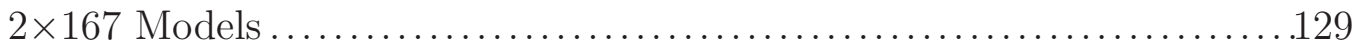

Potential Sources for Complications in Modeling Data ......................129

$4 \times 167$ gH5 Array Structure Properties ..................................134

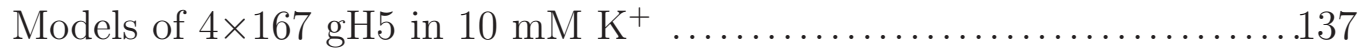

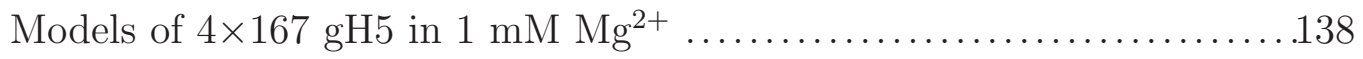

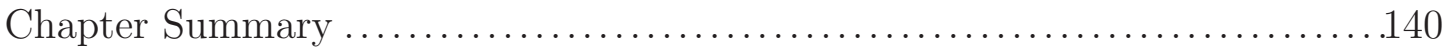




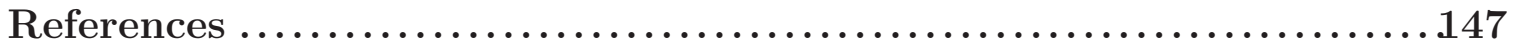

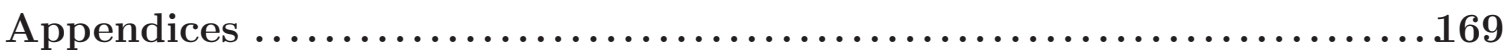

A: Supplemental data for "Elucidating Internucleosome Interactions and the

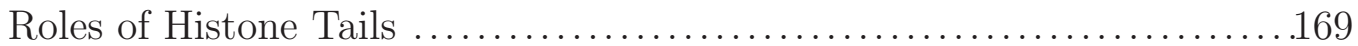

Supporting Data to the Results Presented in the Main Text.............170

Modeling the Structure Factor with a Two-DH Inter-NCP Interaction Potential ..................................................

B: Supplemental Material for SAXS Experiments of Nucleosome Arrays ......181

Concentration Adjustment .......................................... 181

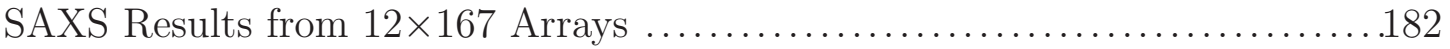

C: CG MC Simulation Algorithm for ds-DNA Supplementary Material........184 


\section{List of Figures}

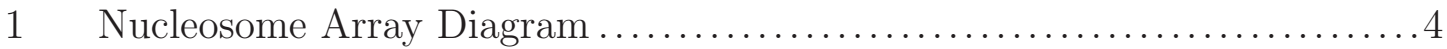

2 Nucleosome Core Particle (PDB ID: 1KX5) ....................... 6

3 Annotated Diagram of a Tetranucleosome Model (PDB ID: 1ZBB) ......10

4 Effective Length Scales of Different Biological Techniques................10

$5 \quad$ Sample Environment of Structural Biology Probes.......................11

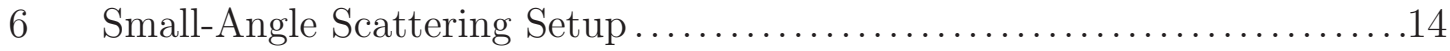

7 Scattering intensity is the product of the structure factor and the form

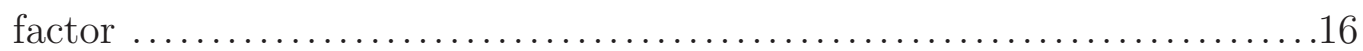

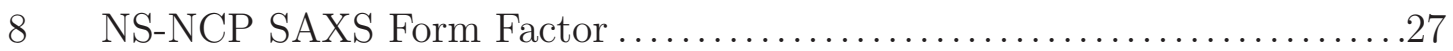

9 SAXS Profiles and GOCM Fits of NS-NCP in a Series of KCl Concen-

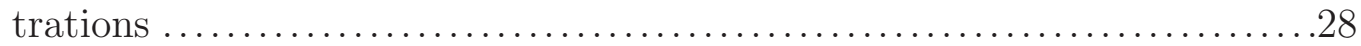

10 SAXS Profiles and GOCM Fits of gH3 and gH4 RC-NCPs in 10 and

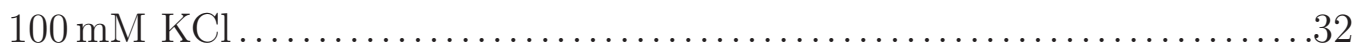

11 SDS-PAGE Gel of the Histone Proteins...............................37

12 Agarose Gel of the Purified DNA Templates ..........................38

13 SDS-PAGE Gel of the Combined Histone Proteins ......................40

14 Example Agarose Gel from Differential Centrifugation ..................41

15 Raw Sample and Buffer Scattering Profiles ...........................43

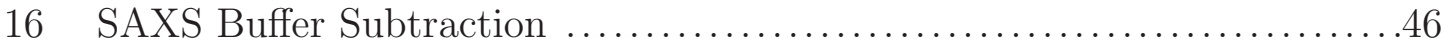

17 Zero Concentration Extrapolation for $2 \times 167$ Arrays....................49

18 Zero Concentration Extrapolation for $3 \times 167$ Arrays....................50

19 Zero Concentration Extrapolation for $4 \times 167$ Arrays.....................51

20 Zero Concentration Extrapolation for $4 \times 167 \mathrm{gH} 5$ Arrays ................52

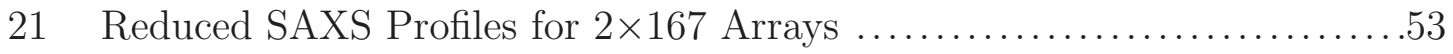

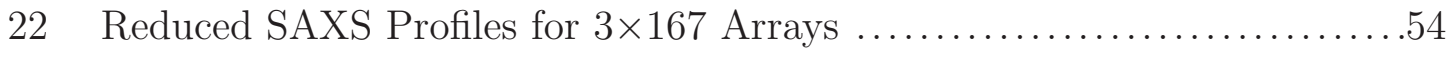

23 Reduced SAXS Profiles for $4 \times 167$ Arrays ...........................55 
24 Reduced SAXS Profiles for $4 \times 167$ gH5 Arrays .......................56

25 Comparison of the Nucleosome Array Guinier Fit Results ..............57

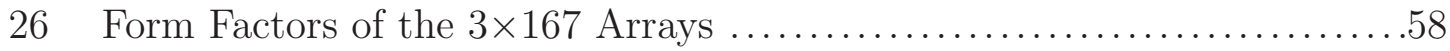

27 Pair-Distance Distributions from the $3 \times 167$ Arrays ...................59

28 Form Factors of the $4 \times 167$ Arrays ..................................61

29 Pair-Distance Distributions from the $4 \times 167$ Arrays .....................62

30 Form Factors for Nucleosome Arrays of Varied Length ..................63

31 Pair-Distance Distributions for Nucleosome Arrays of Varied Length .....64

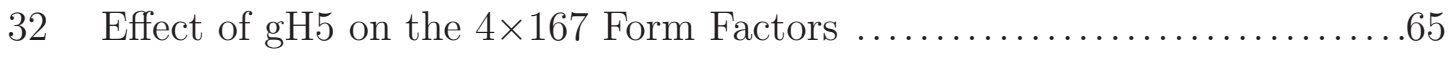

33 Pair-Distance Distributions from the $4 \times 167$ gH5 Arrays ................66

34 Form Factor Comparison ........................................67

35 Geometric Definitions for the Tetranucleosome Array ...................70

36 NCP Opening Angle Rigid Body Moves ...........................72

37 Best-Matched Models from Opening Angle Moves ......................72

38 Twist Rigid Body Moves of $\mathrm{NCP}_{2}$ and $\mathrm{NCP}_{4} \ldots \ldots \ldots \ldots \ldots \ldots \ldots \ldots \ldots$

39 Best-Matched Models from Twist Moves of $\mathrm{NCP}_{2}$ and $\mathrm{NCP}_{4}: \ldots \ldots \ldots . . .74$

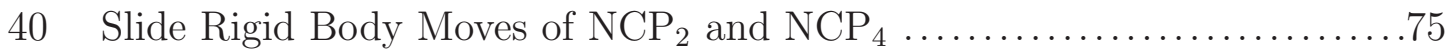

41 Best-Matched Models from Slide Moves of $\mathrm{NCP}_{2}$ and $\mathrm{NCP}_{4} \ldots \ldots \ldots \ldots . \ldots 6$

42 Slide Moves of All NCPs ........................................ 76

43 Best-Matched Models from Slide Moves of All NCPs ....................77

44 Scan of Rotation Angles.......................................... 77

45 Illustration of the Bead-Rod Model ................................85

46 Schematic of the DNA Monte Carlo Algorithm ........................89

47 Bulk Properties of the Wormlike Bead-Rod Model .....................92

48 Survey of Simulated DNA Twist Angles .............................93

49 Schematic of a DNA Molecule ...................................94

50 Raw versus Energy Minimized Dihedral Angles ........................96 
51 Percent of Acceptable Dihedral Angles...............................97

52 Example Mononucleosome Simulation .............................101

53 Example Tetranucleosome Simulation ...............................104

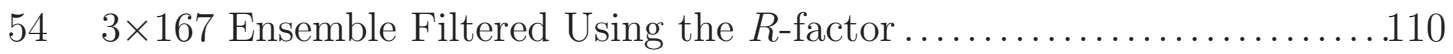

$553 \times 167$ Ensemble Filtered Using the Weighted $R$-factor...............112

$563 \times 167$ Ensemble Filtered Using the $F$-factor ......................114

$573 \times 167$ Ensemble Filtered Using the $\chi^{2}$ Statistic ....................115

$584 \times 167$ Sub-Ensembles for the $10 \mathrm{mM} \mathrm{K}^{+}$and $1 \mathrm{mM} \mathrm{Mg}^{2+} 4 \times 167 \mathrm{gH} 5$

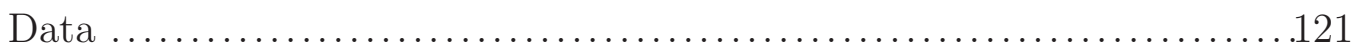

$594 \times 167$ Sub-Ensembles for the 10 and $50 \mathrm{mM} \mathrm{K}^{+}$Data .................123

$604 \times 167$ Sub-Ensembles for the $100 \mathrm{mM} \mathrm{K}^{+}$and $1 \mathrm{mM} \mathrm{Mg}^{2+}$ Data .......124

$613 \times 167$ Sub-Ensembles for the 10 and $50 \mathrm{mM} \mathrm{K}^{+}$Data ................127

$623 \times 167$ Sub-Ensembles for the 100 and $200 \mathrm{mM} \mathrm{K}^{+}$Data................128

$632 \times 167$ Sub-Ensemble for the $10 \mathrm{mM} \mathrm{K}^{+}$Data.......................130

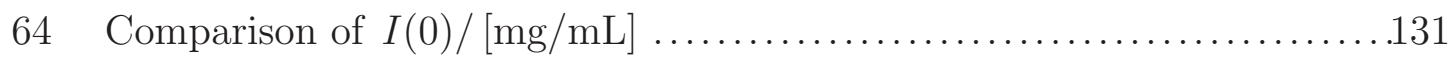

$654 \times 167 \mathrm{gH} 5$ Sub-ensembles of the Best 1000 Models ...................135

66 Comparison of a Sample $1 \mathrm{mM} \mathrm{Mg}^{2+} 4 \times 167$ gH5 Model to the $1 \mathrm{ZBB}$

Model ........................................................... 139

67 Characterization of NCP Samples ...............................170

68 Form Factors the Mononucleosomes Constructs ......................171

69 SAXS Profiles of NS-NCPs in a Series of $\mathrm{MgCl}_{2}$ Concentrations .........172

70 SAXS Profiles and GOCM Fits of gH3 and gH4 RC-NCPs in 20 and

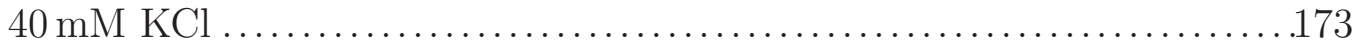

71 SAXS Profiles and GOCM fits of Intact Recombinant RC-NCPs in

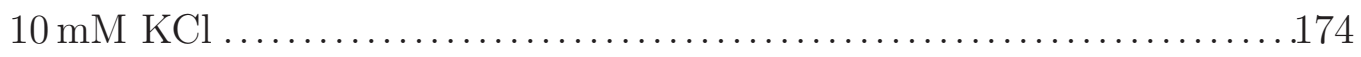

72 SAXS profiles of NS-NCPs in a Series of $\mathrm{KCl}$ concentrations with fits based on the two-DH inter-NCP potential ........................177 
73 SAXS profiles of $\mathrm{gH} 3$ and $\mathrm{gH} 4 \mathrm{NCPs}$ in 10 and $100 \mathrm{mM} \mathrm{KCl}$ with fits based on the two-DH inter-NCP potential ........................178

74 SAXS profiles of gH3 and gH4 NCPs in 20 and $40 \mathrm{mM} \mathrm{KCl}$ with fits based on the two-DH inter-NCP potential ......................... 179

75 SAXS profiles of RC-NCPs in $10 \mathrm{mM} \mathrm{KCl}$ with fits based on the two-

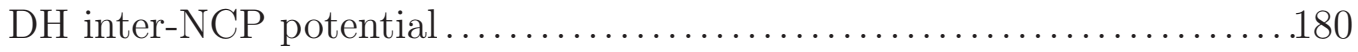

76 Sample Concentration Refinement.................................181

77 Reduced SAXS Profiles for $12 \times 167$ Nucleosome Arrays .................182

78 Zero Concentration Extrapolation for $12 \times 167$ Samples ...............183

79 DNA Dihedral Angle $\alpha$ from the 75 ns MD simulation .................185

80 DNA Dihedral Angle $\beta$ from the 75 ns MD simulation ..................185

81 DNA Dihedral Angle $\gamma$ from the 75 ns MD simulation .................186

82 DNA Dihedral Angle $\delta$ from the 75 ns MD simulation .................186

83 DNA Dihedral Angle $\varepsilon$ from the $75 \mathrm{~ns}$ MD simulation ................187

84 DNA Dihedral Angle $\zeta$ from the $75 \mathrm{~ns}$ MD simulation ...............187

85 DNA Dihedral Angle $\chi$ from the 75 ns MD simulation ................188

86 Iterative Minimization of dsDNA ..................................... 189

87 Spatial Range of the Complete vs Best Ensembles .....................190

88 ConveNCP NCP in the Tetranucleosome Array Ensemble ...............191 


\section{List of Tables}

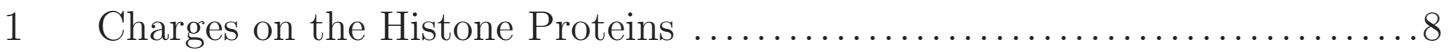

2 Inter-NCP Interaction Parameters............................... 30

3 Summary of DNA Dihedral Distributions........................... 95

4 Summary of Simulation Parameters ................................ 98

$5 \quad R_{g}$ and $I(0)$ Calculated from the Guinier Fit Versus the $P(r) \ldots \ldots \ldots .133$

$6 \quad$ Stacked NCP Features from the Best $10004 \times 167 \mathrm{gH} 5$ Models ...........136

$7 \quad$ Adjacent NCP Features from the Best 1000 4×167 gH5 Models ..........138 


\section{List of Symbols}

$\alpha \quad$ DNA dihedral angle formed by the atoms: $\mathrm{O} 3^{\prime}-\mathrm{P}-\mathrm{O} 5^{\prime}-\mathrm{C} 5^{\prime}$

$\beta \quad$ DNA dihedral angle between the atoms: $\mathrm{P}-\mathrm{O} 5^{\prime}-\mathrm{C} 5^{\prime}-\mathrm{C} 4^{\prime}$

$\beta(Q)$ measure of the level of polydispersity and spherical symmetry of a particle

c speed of light

$c_{\text {scale }} \quad$ scale constant used to scale a model and experimental data set

$\gamma \quad$ DNA dihedral angle between the atoms: $\mathrm{O} 5^{\prime}-\mathrm{C} 5^{\prime}-\mathrm{C} 4^{\prime}-\mathrm{C} 3^{\prime}$

$\Gamma \quad$ gamma function

$\delta \quad$ DNA dihedral angle between the atoms: $\mathrm{C}^{\prime}-\mathrm{C} 4^{\prime}-\mathrm{C} 3^{\prime}-\mathrm{O} 3^{\prime}$

$D_{\max }$ maximum distance between atoms in a particle

$D_{\text {order }}$ length of ordering

$D_{\text {repeat }}$ distance between repeated structure features

$\epsilon \quad$ dielectric constant of water

$\varepsilon \quad$ DNA dihedral angle between the atoms: C4'-C3'-O3'-P

E beam energy

F $\quad$-factor (fitness function)

$\mathcal{F} \quad$ Fourier transform

$\mathcal{F}^{-1} \quad$ reverse Fourier transform

h Plank constant 
$I_{e} \quad$ experimental scattering intensity

$I_{j} \quad$ scattering intensity of pixel $j$

$I_{m} \quad$ theoretical scattering intensity of a model structure

$I(Q) \quad$ scattering intensity as a function of momentum transfer

$I^{\prime}(Q) \quad I(Q)$ transformed using the Fourier transform then transformed back with the reverse Fourier transform

$I_{Q_{\text {bin }}} \quad$ scattering intensity averaged from all pixels in a specific $Q_{\text {bin }}$

$\kappa \quad$ harmonic twist force constant between DNA bp

$\kappa_{\text {attr }} \quad$ inverse Debye screening length for attraction

$k_{B} \quad$ Boltzmann constant

$\kappa_{D} \quad$ inverse Debye screening length for repulsion

$l \quad$ length of inextensible rod connecting beads in discrete wormlike chain

$L \quad$ total contour length of a wormlike chain

$\lambda \quad$ X-ray or neutron beam wavelength

$L_{p} \quad$ persistence length of DNA

$n \quad$ number of degrees of freedom

$N_{e} \quad$ number of repeated exposures

$N_{g} \quad$ number of grid points used in the reciprocal space convergence

$n_{p} \quad$ average number density of particles

$N_{Q} \quad$ number of $Q$-points 


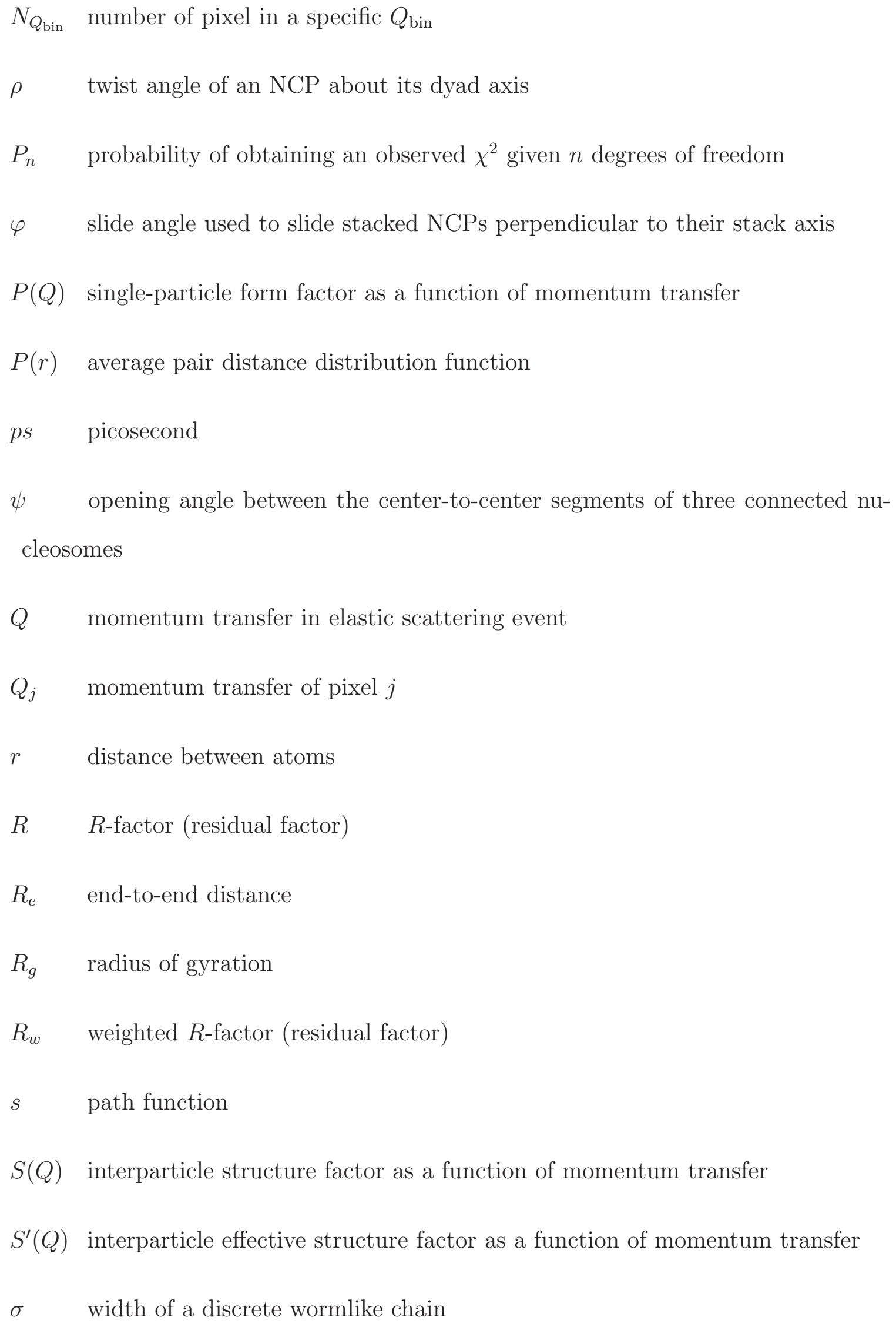


$\sigma_{j} \quad$ error in the scattering intensity of pixel $j$

$\sigma_{N} \quad$ equivalent $\mathrm{NCP}$ diameter

$\sigma_{\text {peak }} \quad$ full width at half maximum for a Bragg peak

$\sigma(Q) \quad$ error in the scattering intensity as a function of momentum transfer

$\sigma_{Q_{\text {bin }}}$ error in the scattering intensity averaged from all pixels in a specific $Q_{\text {bin }}$

$\sigma_{\mathrm{SD}} \quad$ standard deviation

T temperature

$\vartheta \quad$ half of the scattering angle

$\delta \vartheta_{\max }$ maximum sampling angle for DNA MC steps

$u \quad$ unit vector between continuous segments of a wormlike chain

$U_{\text {bend }}$ bending energy of a wormlike chain

$U_{\mathrm{HS}} \quad$ hard-sphere potential

$u_{k} \quad$ unit vector of the $k^{\text {th }}$ rod in a discrete wormlike chain

$U(r)$ inter-NCP interaction potential as function of inter-NCP distance

$U_{\text {WCA }}$ Weeks-Chandler-Andersen form of a purely repulsive Lennard-Jones potential

$\omega \quad$ twist angle between DNA bp

$w_{i} \quad$ weight factor used in merging different concentration data

$\chi \quad$ DNA dihedral angle between the atoms: O4' $^{\prime}-\mathrm{C1}^{\prime}-\mathrm{N} 1 / \mathrm{N} 9-\mathrm{C} 4 / \mathrm{C} 2$

$\chi^{2} \quad$ chi-squared statistic

$Z_{\text {attr }}$ effective NCP attractive charge 
$Z_{\text {bare }}$ bare NCP charge

$Z_{\text {eff }} \quad$ effective NCP repulsive charge

$Z_{\text {ren }} \quad$ renormalized effective NCP charge

$\zeta \quad$ DNA dihedral angle between the atoms: C3'-O3'-P-O5' 


\section{Glossary of Terms and Abbreviations}

1D: one-dimensional

1KX5: protein data bank identifier for the atomistic structure model of the crystal structure of the nucleosome core particle [1]

1ZBB: protein data bank identifier for the atomistic structure model of the crystal structure of the tetranucleosome [2]

2D: two-dimensional

3D: three-dimensional

A: Angstrom, $10^{-10}$ meter

a.a.: amino acid

API: application programming interface

array center: the midpoint between the centers of the four nucleosomes in a tetranucleosome array

BI DNA: B-form DNA base for which $\epsilon-\zeta>0$

BII DNA: B-form DNA base for which $\epsilon-\zeta<0$

bp: DNA base pair

${ }^{\circ} \mathrm{C}$ : degrees Celsius

$\mathrm{Ca}^{2+}$ : calcium cation

CG: coarse-grain

cm: centimeter, $10^{-2}$ meter 
CHESS: Cornell High Energy Synchrotron Source

C-ter: carboxyl-terminus, or C-terminal, end of a protein chain

cryo-EM: cryogenic electron microscopy

CRYSOL: a program to calculate theoretical X-ray solution scattering of biological macromolecules from atomic coordinates

Da: Dalton, equal to one atomic mass unit, the mass of a hydrogen atom.

DAMMIN: a program to restore ab initio low resolution shape of randomly oriented particles in solution

DH: Debye-Hückel

DNA: deoxyribonucleic acid

dsDNA: double-stranded deoxyribonucleic acid

e: elementary charge, or electric charge carried by a single proton, $1.60217662 \times 10^{-19}$ coulombs

E. coli: Escherichia coli bacteria

FWHM: full width at half maximum

G1/CHESS: G1 beamline at the Cornell High Energy Synchrotron Source

gH1: globular variant of histone $\mathrm{H} 1$

gH3: globular variant of histone $\mathrm{H} 3$

gH4: globular variant of histone $\mathrm{H} 4$

gH5: globular variant of histone H5

GOCM: generalized one component method 
H1: nucleosome linker histone

H2A: nucleosome core histone

H2B: nucleosome core histone

H3: nucleosome core histone

H4: nucleosome core histone

H5: nucleosome linker histone

HS: hard-sphere

$\mathrm{K}^{+}$: potassium cation

kDa: kilodalton, $10^{3}$ Dalton

$\mathrm{keV}$ : kiloelectron volt, $10^{3}$ electron volt, unit of energy

$\mathrm{km}$ : kilometer, $10^{3}$ meter

LB: lysogeny broth

m: meter, unit of distance

M: moles per liter, molar concentration or molarity

MC: Monte Carlo

MD: molecular dynamics

MDa: megadalton, $10^{6}$ Dalton

mg: milligrams, unit of mass

$\mathrm{Mg}^{2+}$ : magnesium cation

min: minute 
mL: milliliters, unit of volume

$\mu \mathrm{m}$ : micrometer, $10^{-6}$ meter

mm: millimeter, $10^{-3}$ meter

mM: millimolar, $10^{-3}$ moles per liter

MMTV: mouse mammary tumor virus, the MMTV DNA sequence acts as a nucleosome positioning sequence but with a lower binding affinity compared to the W601 sequence

mononucleosome: a single nucleosome

N-ter: amino-terminus, or N-terminal, end of a protein chain

NAMD: nanoscale molecular dynamics, a molecular dynamics simulation program [3]

NCP: nucleosome core particle, a nucleosome with only the 147 bp of dsDNA wrapping the histone protein core (when we refer to an NCP as part of a nucleosome array, we are referring to a nucleosome separate to any linker DNA)

NCP center: the intersection of the NCP cylinder axis and the NCP dyad axis

$\mathrm{NCP}$ cylinder axis: the center axis of a cylinder fit to the $\mathrm{C}^{\prime}$ atoms of an NCP

NCP dyad: the central DNA bp of an NCP (the $74^{\text {th }}$ bp of a fully wrapped NCP)

NCP dyad axis: the axis orthogonal to the NCP cylinder axis that passes through the center of the NCP dyad

NCP face: treating an NCP as a cylinder, the flat surfaces would be the face

NCP side: treating an NCP as a cylinder, the round surface would be the side

$\mathrm{NCP}_{i}$ : the $i^{\text {th }} \mathrm{NCP}$ in an nucleosome array; $\mathrm{NCP}_{1}$ is the nucleosome closest to the $5^{\prime}$ end of the template DNA 
NG-3: SANS beamline at the National Institute of Standards and Technology Center for Neutron Research

nm: nanometer, $10^{-9}$ meter

NMR: nuclear magnetic resonance imaging

NS-NCP: natural-source NCP, these NCPs were extracted from chicken erythrocytes NSLS: National Synchrotron Light Source, Brookhaven National Laboratory

nucleosome: molecular building block of chromatin, DNA-protein complex containing 2 copies each of core histones H2A, H2B, H3, and H4, wrapped 1.65 times by dsDNA. The difference between a nucleosome and a nucleosome core particle is that the nucleosome contains more that the minimum 147 bp dsDNA.

$N \times 167$ : notation for referring to a nucleosome array with $N$ repeats of the W601 nucleosome positioning sequence connected by 20 bp of linker dsDNA

opening angle: the angle between the center-to-center segments of three connected nucleosomes

PAGE: polyacrylamide gel electrophoresis

PDB: Protein Data Bank file containing the coordinates of a molecular structure

PSF: Protein Structure File containing the bond information for a molecular structure

PSFGEN: PSF generator, a program to generate a PDB and PSF pair used to fully define a molecular structure's coordinates and bond information respectively

RC-NCP: recombinant NCP

rpm: revolutions per minute 
[salt]: Square brackets around a quantity is a common mechanism to denote the concentration of that quantity, often used for referring to a salt or mass concentrations. In this example, [salt] represents the salt concentration.

SANS: small-angle neutron scattering

SAS: small-angle scattering

SASSIE: small angle scattering systematic integrated environment, a program developed at the NIST Center for Neutron Research to model intrinsically disordered biological molecules

SAXS: small-angle X-ray scattering

SDS: sodium dodecyl sulfate

SNR: signal-to-noise ratio

ssNA: single-stranded nucleic acid

stack axis: the line pointing from the center of $\mathrm{NCP}_{i}$ to the center of $\mathrm{NCP}_{i+2}$

tetranucleosome: an array of four connected nucleosomes

twist angle: the dihedral angle between $\mathrm{NCP}_{i}$ and $\mathrm{NCP}_{i+1}$, formed by the center-tocenter segment between NCPs and the two cylinder axes

UV: ultraviolet

$\mathrm{V}$ : volt, electric potential

W601: Widom 601 DNA sequence [4] know to strongly bind and therefore position nucleosomes

WAXS: wide-angle X-ray scattering 
WCA: Weeks-Chandler-Andersen

X9/NSLS: X9 beamline at the National Synchrotron Light Source, Brookhaven National Laboratory 
"If our small minds, for some convenience, divide this ... universe, into parts-physics, biology, geology, astronomy, psychology, and so on-remember that nature does not know it!"

- Richard P. Feynman, 1963

"Focus too closely on the goal you haven't accomplished, and you'll fail to notice the victories you achieve along the way."

-Orson Scott Card, "Earth Awakens" 


\section{Chapter 1: Introduction and Background}

\section{Dissertation Outline}

Deoxyribonucleic acid (DNA) encodes the genetic information essential for life. This genetic code controls the life cycle of living organisms at the fundamental level. How is the expression of DNA regulated? How does the structure of DNA packaging impact that regulation? These two questions endure as the most fundamental, and as yet unanswered questions of biology [5], the details of which have immense complexity [6]. The goal of my research is to illuminate the dynamic conformations of packaged DNA, specifically nucleosomes and nucleosome arrays, using small-angle X-ray scattering (SAXS). By filtering ensembles of atomic model structures against experimental SAXS profiles, we have generated sub-ensembles that agree with experimental solution scattering of nucleosome arrays. From the atomic structures in these sub-ensembles we extract key characteristics of the packaging of nucleosome arrays.

The background information in Chapter 1 motivates this line of research and the selection of SAXS as the best suited experimental technique. Chapter 2 contains the foundational studies probing the structure and interaction of nucleosome monomers measured using SAXS. We quantify the interaction potentials for mononucleosomes and compare the interaction of nucleosomes with the removal of the $\mathrm{H} 3$ or $\mathrm{H} 4$ histone protein tails. Chapter 3 presents the SAXS profiles from arrays containing 2, 3, 4, and 12 nucleosomes (referred to as $2 \times 167,3 \times 167,4 \times 167$, and $12 \times 167$ arrays as the repeated DNA length is 167 basepairs) in a variety of salt concentrations. SAXS profiles of $4 \times 167$ arrays with globular linker histone H5 (gH5) are also reported for select salt concentrations (denoted as [salt]). The similarities between the $4 \times 167$

crystal structure [2] and the experimental results from arrays with linker histones motivate manually transforming the atomic crystal structure to explore the difference. To model less similar structures we developed a coarse-grain (CG) Monte Carlo (MC) 
simulation algorithm for sampling DNA structures to generate ensembles of atomic models, the details of which are discussed in Chapter 4. Chapter 5 shows the results from applying this algorithm to generate ensembles of nucleosome arrays models which agree with the experimental SAXS results from Chapter 3. A brief summary and discussion on future direction in Chapter 6 concludes this dissertation. Appendices provide supplemental information for the mononucleosome, and nucleosome array measurements, as well as the CG DNA simulation algorithm. An additional appendix documents the Python code developed for preparing, analyzing, and presenting model nucleosome structure results.

Portions of this research have been published or are in preparation for publication. As the manuscripts succinctly report the essential details, we merge them directly into this document where they best serve the narrative. This aims to make the context of the publications evident while highlighting the common theme. Unpublished results are explained more thoroughly with the expectation that they will be published soon. This is particularly true for the ensembles results of nucleosome arrays contained in Chapters 3 and 5 .

\section{Chromatin: DNA in Eukaryotic Cells}

The majority of human cells contain $6 \times 10^{9}$ base pairs (bp) of DNA packaged as 23 pairs of chromosomes which would span two meters if arranged in a straight line. All the DNA in a human body placed end-to-end would be $100 \times 10^{9} \mathrm{~km}$ or roughly 670 times the distance from the Earth to the Sun [7]. Consequently, intricate packing mechanisms control the arrangement of the $2 \mathrm{~m}$ of DNA within each cell nucleus, which has a diameter on the order of $10 \mu \mathrm{m}$.

Packaged DNA within the cell forms chromatin, a DNA-protein complex which serves to compact, organize, and protect DNA while also facilitating genetic expression. The nucleosome core particle (NCP) is the molecular building block of chro- 


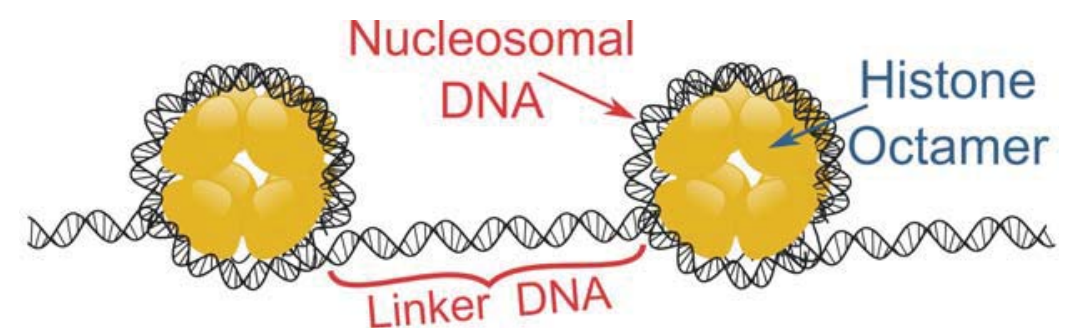

Figure 1. Nucleosome Array Diagram: Illustration of a nucleosome array with pertinent components identified.

matin and is composed of $147 \mathrm{bp}$ of DNA which wrap 1.65 times around a symmetric octamer of histone proteins. ${ }^{1}$ Each NCP is linked by 10-90 bp of DNA [8] as "beadson-a-string", illustrated in fig. 1. The structure and packaging of these "beads-on-astring" to form chromatin is critically important as it determines the accessibility of the genetic code, and therefore affects genetic expression, replication, and repair [9]. Consequently elaborate regulations of chromatin-related activities occur naturally to establish normal cellular functions $[6,10]$ and better understanding these regulations plays a vital role in understanding fundamental biological processes such as genetic expression, fertility, and viral infectivity [11].

\section{Toward Elucidating Chromatin Structure}

Perturbation of the DNA-chromatin structure impact the accessibility of DNA for transcription and therefore alters genetic expression without changing genetic sequence. As an example, such DNA perturbations were shown to change the genetic expression of fur color and obesity levels in genetically identical mice [12]. These chemical modifications of chromatin, referred to as epigenetic markers, have been shown to be a key component of genetic expression [13]. Epigenetic modifications can occur on the DNA, by means of methylation of the cytosine residue [14], or more commonly on the histone proteins, through more complex processes with dozens of variations and combinations [13]. Reports suggest that, beyond simply packaging

\footnotetext{
1 The difference between a nucleosome and a nucleosome core particle is that the first has linker DNA, i.e, more than $147 \mathrm{bp}$, and the second does not.
} 
DNA, histones function to regulate expression of the genetic code [15-18]. It is believed that these various modifications are recognized by diverse classes of proteins which then lead to suppression or activation of gene expression. The regulation of gene expression has been shown to occur in response to the variation of as little as a single residue on the histone tails in vivo, via post-translational modifications [19-21]. Despite immense efforts to understand the biochemical identity of chromatin, through projects such as the sequencing and annotation of the human genome [22] and the current large-scale mapping of the epigenetic markers [9], this extensive genetic and epigenetic knowledge has yet to yield a universal set of mechanisms describing their relation to gene regulation. It is believed that the chromatin structure may play a role in this regulation $[6,10]$ and may actually be the missing link between the biochemical content and gene regulation; that gene regulation occurs in response to the structure changes resulting from the biochemical modifications of chromatin.

Analogous to how an enzyme's molecular structure determines its enzymatic function, understanding chromatin structure is key in determining its structure-dependent function. At the genomic scale, it remains unclear whether chromatin is folded into highly ordered hierarchical structures or into some non-hierarchical fractal state [23]. On the mesoscopic scale, there are numerous competing models to describe the structure of the "30-nm fiber" of compact chromatin observed in vitro including the onestart solenoid, two-start supercoil, two-start zigzag, and two-start twisted structures [5, 24]. Other reports question the mere existence of the "30-nm fiber" in vivo [25].

This uncertainty in the structure of chromatin persists despite reports using Xray crystallography and cryogenic electron microscopy (cryo-EM) to detail atomic structures of a nucleosome monomer [1] (fig. 2), trimer [26], and tetramer [2, 27]. Despite knowing the static structure of nucleosome arrays, these methods require conditions drastically different from the cellular environment and it is not clear what effects result from these artificial conditions. Additionally, the diversity of arrays 
studied using crystal diffraction and cryo-EM are limited and an increased variety of arrays, particularly those with histone modifications, must be examined to identify how nucleosome arrays respond to epigenetic modifications.

Detailed results from solution studies of nucleosomes are confined to the complexity of single nucleosome and limited in their scope [28, 29]. From studies of mononucleosomes, the flexible tails of the histone proteins are only poorly resolved [14] yet these tails play an important role in nucleosome interactions [30]. For these histone tails, little is known of

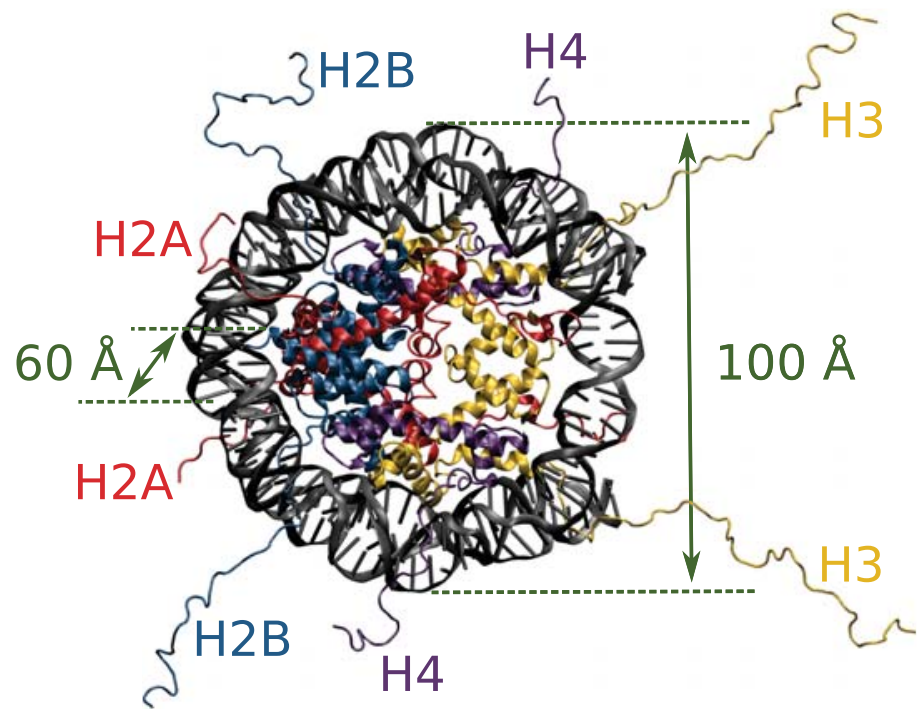

Figure 2. Nucleosome Core Particle (PDB ID: 1KX5): Illustration of the 1KX5 NCP monomer [1] modified to show the extensible histone tails. Each of the two copies of histone proteins H2A, H2B, H3, and $\mathrm{H} 4$ are respectively depicted in red, blue, yellow and purple as labeled. Approximated by a cylinder, an NCP has a diameter of $\sim 100 \AA$ and height of $\sim 60 \AA$.

structurally important dynamic processes comparable to DNA breathing which has been extensively studied [31]. Solution studies of arrays with more nucleosome repeats are primarily qualitative, and do not consider the dynamic solution structure [32]. This lack of quantitative knowledge, both in breadth and depth, of the structure and dynamics of chromatin presents a critical barrier to fully determine its functional roles [23].

\section{Factors Affecting Chromatin Structure}

A myriad of modulating biological and physiochemical factors complicate the acquisition of quantitative knowledge regarding chromatin structure. These factors include 
molecular crowding, cellular ions, truncation of charged regions of the histones, genetic sequence variation, and epigenetic modifications of the histones.

Uniquely in the cellular environment, chromatin behavior is known to change in response to macromolecular solutes which exert a depletion-induced "compression" force referred to as molecular crowding. In vivo, macromolecular solutes occupy 30\% of the nucleus volume [33]. The excluded volume produces pressures as large as tens of atmospheres [34] and, though yet unexplored in vitro, likely produces significant changes on chromatin conformation.

In addition to molecular crowding, cellular ions also modulate chromatin behavior. Inherent with the highly charged nature of the NCP, electrostatics serves as a governing force controlling inter-NCP interactions [35-37]. To understand these interactions, researchers have characterized the effects of cations, with varied valences, on the nucleosome assembly $[35,38,39]$. Examples of this include measuring the sedimentation coefficients of nucleosome arrays and also the fraction of condensed nucleosomes under controlled ionic conditions [40,41]. One notable result from such studies determined that divalent cations go beyond simply screening the electrostatic repulsion of NCPs to induce inter-NCP attraction and even condensation [41] while the same divalent cations, $\mathrm{Mg}^{2+}$ and $\mathrm{Ca}^{2+}$, do not condense DNA [42]. This suggests the importance of the complex charge distribution resulting from the addition of histones in modifying the DNA interactions [43].

The charge distribution on the amino acids of the histone tails contributes to the complex electrostatic interactions of nucleosomes. The octameric histone core of nucleosomes exhibits highly ordered structure, referred to as the "histone fold" motif. In contrast, the structure of the histone tails, $30 \%$ of the histones by mass, are not well understood as they extend from the central structure and are flexible by nature [44, 45]. Though the NCP has a net negative charge, these histone tails account for $+54 e$ of the total $+142 e$ in the histone proteins. Table 1 shows the charge characteristics 
Table 1. Charges on the Histone Proteins: Nucleosome interactions are impacted by the charge distribution of the four natural histone proteins, H2A, H2B, $\mathrm{H} 3$, and H4, and the two mutants globular H3 (gH3) and globular H4 (gH4). Proteins are made from a chain of amino acids (a.a.), contributing $+1 e$ or $-1 e$ to the proteins charge for each basic or acidic a.a. respectively. The values shown are calculated for a neutral $\mathrm{pH}$ solution. The mutant proteins $\mathrm{gH} 3$ and $\mathrm{gH} 4$ are recombinant proteins that respectively lack the first 27 and 10 residues. This truncation removes all the charges from the N-terminal (N-ter) tail of the protein.

\begin{tabular}{|l|lccccccc|}
\cline { 2 - 8 } \multicolumn{1}{c|}{} & $\begin{array}{l}\text { Histone } \\
\text { Protein }\end{array}$ & $\begin{array}{c}\text { Amino-Acid } \\
\text { Residues }\end{array}$ & $\begin{array}{c}\text { Basic } \\
\text { a.a. }\end{array}$ & $\begin{array}{c}\text { Acidic } \\
\text { a.a. }\end{array}$ & $\begin{array}{c}\text { Global } \\
\text { Charge }\end{array}$ & \multicolumn{2}{c}{ Tail Charges } \\
Natural & H2A & $1-129$ & 24 & 9 & +15 & N-ter: 4 & C-ter: 1 & 13950.2 \\
& & & & & & C-ter: 3 & & \\
& H2B & $1-122$ & 28 & 9 & +19 & N-ter: 8 & N-ter: 1 & 13493.6 \\
& H3 & $1-135$ & 30 & 11 & +19 & N-ter: 10 & & 15270.8 \\
& H4 & $1-102$ & 25 & 7 & +18 & N-ter: 3 & & 11236.1 \\
\hline Mutants & gH3 & $28-135$ & 21 & 11 & +9 & & & 12378.4 \\
& gH4 & $11-102$ & 22 & 7 & +15 & & & 10338.1 \\
\hline
\end{tabular}

of the four different proteins that make up the symmetric histone octamer composed of two copies each of $\mathrm{H} 2 \mathrm{~A}, \mathrm{H} 2 \mathrm{~B}, \mathrm{H} 3$, and $\mathrm{H} 4$.

Two additional factors in chromatin conformation and dynamics are the genetic variation, both of the nucleosomal and linker DNA, as well as epigenetic variations of the histones. The sequence of DNA bases directly influences nucleosome positioning and sliding, DNA breathing, and linker DNA length [46-48]. The linker DNA, in particular, acts as a steric constraint by setting the distance and twist angle $\left(\sim 360^{\circ}\right.$ per $10 \mathrm{bp}$ ) between adjacent nucleosomes thereby limiting the possible conformations [49].

For histone variations, the positively charged flexible tails have been shown to be crucial in modulating nucleosome interaction [38]. Each of the eight histone proteins has an N-terminal and a C-terminal tail for a total of 16 sites for mutation. In an effort to dissect the contribution from each of the histone tails, Gordon et al. studied the 16 possible single-tail-deletion NCP mutants and determined that the N-terminal tails are most important to the condensation of oligo-NCP [50]. This same study 
also showed that removing the eight N-terminal tails, which hold the most positive charge, led to the NCPs remaining soluble in high divalent salt solutions. In vitro, the acetylation of histone $\mathrm{H} 4$ lysine 16 (H4K16), which is essential for activating DNA transcription in vivo [51], has been shown to decondense nucleosome arrays and allow enzymatic access [52-54]. Acetylation of other lysine residues on the H4 tail, in particular $\mathrm{H} 4 \mathrm{~K} 5, \mathrm{H} 4 \mathrm{~K} 8$, and $\mathrm{H} 4 \mathrm{~K} 12$, produced much less change indicating the combination of both non-specific (electrostatic) and specific roles of the histone tails $[52]$.

In addition to this experimental work, there have been in-depth theoretical studies investigating chromatin conformation in an effort to complement experimental results. Molecular dynamic (MD) simulations have been the primary technique for investigating the complex intra- and inter-NCP interactions and the bending and twisting of linker DNA in chromatin assembly $[5,55,56]$. Larger systems of NCP arrays and chromatin require $\mathrm{CG}$ approaches in order to adequately reduce the computational cost; these CG simulations have qualitatively reproduced the observed cation-induced NCP array folding and sedimentation coefficients [57]. Mesoscale models, developed by Arya and Schlick, also include the effect of linker histones [49], another histone protein known to nonspecifically bind to nucleosome DNA [58] further facilitating compaction. Genome-scale models, employing two-angle approximations, predict accessible phase diagrams for chromatin $[59,60]$. In light of the controversy regarding chromatin's true structure, one important observation from diverse computational studies is the polymorphic nature of compact chromatin structure [5], suggesting the possibility of non-unique conformations. With all these developments, experimental corroboration is sorely needed to validate and refine these predictive models.

\section{Experimental Biological Probes}


Before investigating a system, two important factors to consider are the length scale of the system compared to the probe and the environmental restrictions of the probe. Solution small-angle scattering (SAS) is an experimental technique well suited to probe the structure of NCP arrays at mesoscopic scales

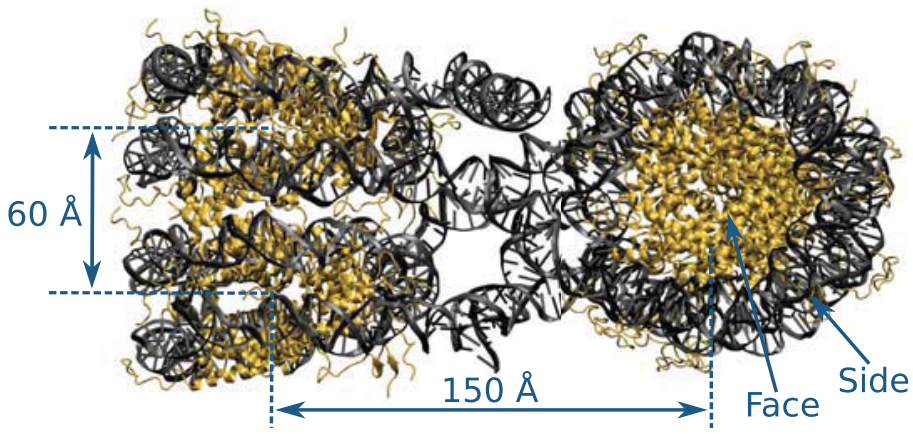

Figure 3. Annotated Diagram of a Tetranucleosome Model (PDB ID: 1ZBB): In the tetranucleosome crystal structure [2], $\mathrm{NCP}_{i}$ and $\mathrm{NCP}_{i+2}$ are stacked in a face-on-face orientation. The center-to-center distance between stacked NCPs is $\sim 60 \AA$ and the NCP stacks are separated by $\sim 150 \AA$.

in an environment that mimics cellular conditions.

When probing any system, the size of the probe determines the length scale of the structural information obtained. The wavelength of visible light $(\sim 380-740 \mathrm{~nm})$, for example, makes optical microscopy an appropriate method for studying objects at least as large as a cell (tens of $\mu \mathrm{m})$. As illustrated in figs. 2 and 3, a nucleo-

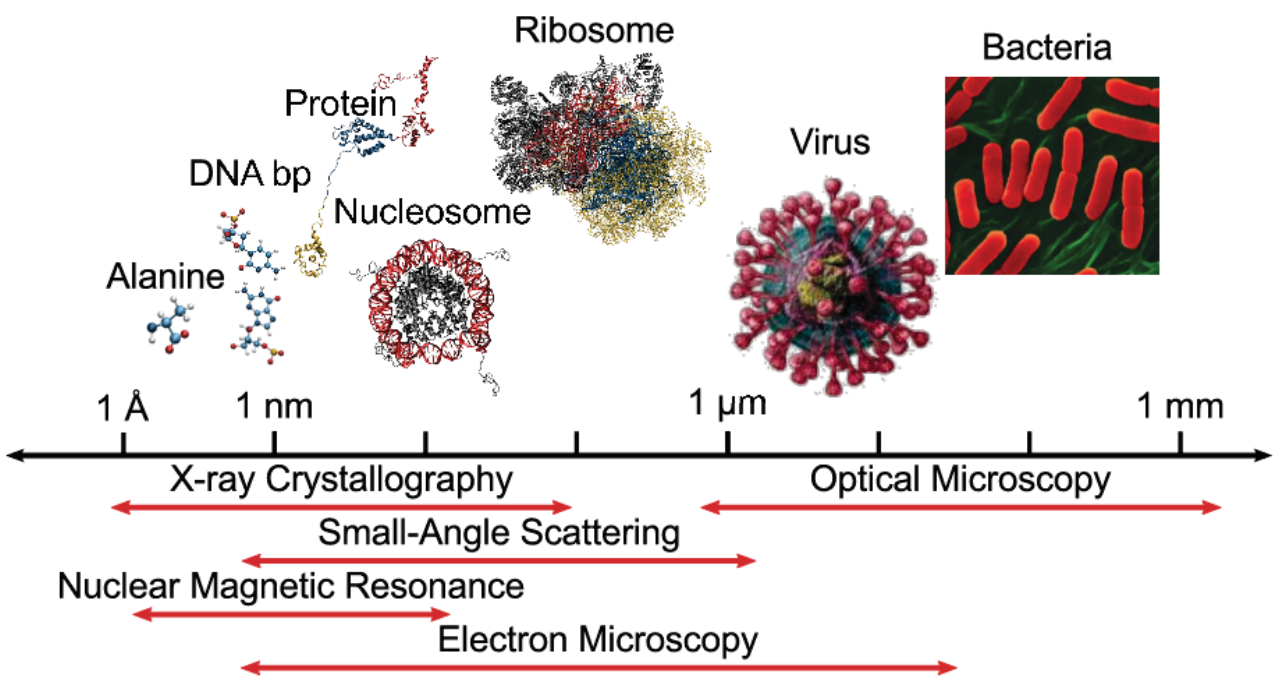

Figure 4. Length Scales of Structural Biology Probes: The various experimental structural biology probes are each sensitive within a specific size range. This range sets the limit on the extent of their effectiveness for studying various systems. Smallangle scattering effectively probes length scales between $1 \mathrm{~nm}$ and $1 \mu \mathrm{m}$. Adapted from Zuo et al. [61]. 
some and nucleosome array has dimensions on the order of $60-500 \AA$. With these dimensions, we see in fig. 4 that several experimental structural biology methods are dimensionally suited to studying nucleosome and nucleosome arrays, including X-ray crystallography, cryo-EM, SAS, and-potentially-nuclear magnetic resonance imaging (NMR).

In addition to probing the correct dimensions, every experimental method examines biological structures in a specific sample environment, the details of which determine the type of information gained and the restrictions on preparing highquality samples. Figure 5 illustrates a comparison between the four complimentary

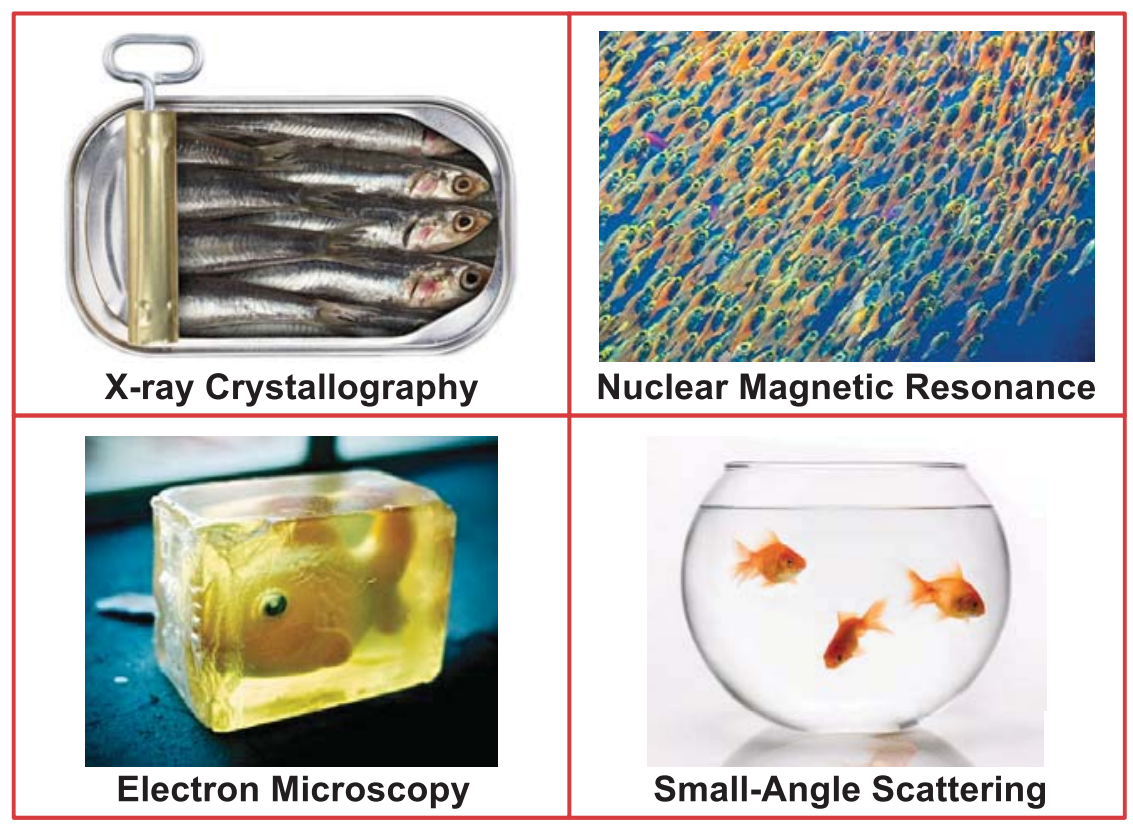

Figure 5. Sample Environment of Structural Biology Probes: The various structural biology probes each examine samples prepared under certain environmental conditions. Such conditions affect the type of information to be gained as well as impose requirements for preparing a high-quality sample. X-ray crystallography requires samples be crystallized and provides detailed information on the regularities in the sample crystals. Electron microscopy similarly requires samples be in a rigid artificial environment. Nuclear magnetic resonance examines biological molecules in a dynamic setting but the samples must have a low molecular weight in order to extract useful information from the sample. Small-angle scattering also probes dynamic macromolecules in solution but the quality of the result corresponds to the purity of the sample solution. 
methods which appropriately probe the correct dimension for nucleosomes: X-ray crystallography, electron microscopy (e.g., cryo-EM), NMR, and SAS.

Crystallography has proven successful in examining nucleosomes [2, 26, 62] but the packing interactions required to form a crystal imposes a dense molecular conformation. X-ray crystallography requires a molecule be prepared in a crystalline solid, a process that is not possible for all biological structures or may require extensive exploration to identify crystallization conditions. When obtainable, the symmetry of such crystals together with systematic analysis allows one to obtain a three-dimensional (3D) structure of the material crystallized. A clear drawback from this technique is that the crystallization process imposes a compact molecular structure, and further, the compaction interactions could potentially modify structural components. Nucleosomes are expected to have dynamics conformations with potentially intrinsic disorder, behavior that is eliminated by crystallization. In addition, successfully crystallizing a large number of structural variations would be time prohibitive.

Cryo-EM provides more flexibility in sample variation as the process of preparing a sample involves preserving the biological molecule in an amorphous ice by rapidly freezing the sample using liquid ethane [63]. Unlike crystallography, cryo-EM provides a density map which are typically noisy and require averaging to smooth the noise. Often the atomic structure of subunits from the complete molecule are then docked to the density map through an optimization process to provide an atomic level rendition of the molecule, as was done for a tetranucleosome array with 40 bp of linker DNA [27]. This has led to cryo-EM typically being used to study larger systems composed of several subunits which have individually been studied using crystallography, Similar to crystallography, cryo-EM introduces an artificial environment with the potential to modifying the structure. More importantly, despite capturing the structure of nucleosome conformations frozen in time, cryo-EM would fail to explore the dynamics of these conformations. 
Compared to X-ray crystallography and cryo-EM, NMR has the advantages of studying dynamics of biological molecules in near native physiological conditions with a major drawback being the increased complexity and lower signal-to-noise ratio (SNR) in the data for larger molecules. NMR measures a time average from an ensemble of structures in solution making it better suited for measuring molecules with disordered regions which would prevent crystallization. Macromolecules studied using modern NMR techniques typically have an upper size limit in the range of 50-70 kDa [64] though some proteins with a mass of 1 MDa have been partially studied [65]. This limit results from a decreased SNR caused by larger molecules moving or tumbling at a slower rate. As a single nucleosome has a molecular mass of $206 \mathrm{kDa}$, NMR could potentially provide supplemental information but fully determining conformations of arrays of nucleosomes would not be feasible.

Similar to NMR, SAS also studies an ensemble of biological molecules in solutions near native physiological conditions but is not limited to structures with a size below 50-70 kDa. The variety of samples that can be studied using SAS is not limited to what can be crystallized, rather just what can be prepared in a pure solution. SAS data represents a time and spatial average of all structural conformations of a molecule ensemble and consequently impure samples drastically complicate the analysis and obscure the information content of the data.

SAS uses a collimated beam of either X-rays or neutron (SAXS or SANS) to probe not only the surface structure of a molecule but the internal structures as well. To set the spatial range of the resulting data, one must tune the wavelength, $\lambda$, of the particles in the incident beam by adjusting the beam energy, $E$, according to the de Broglie wavelength,

$$
E=\frac{h c}{\lambda}
$$


where $h$ is the Plank constant and $c$ is the speed of light. Using X-rays with an energy of $\sim 10 \mathrm{keV}$ translates to a wavelength of $\sim 1.2 \AA$, sufficiently small to effectively probe nucleosomes. ${ }^{2}$ The mechanism for scattering depends on the incident particle but is not limited to just the surface of the molecule; X-rays are scattered by the electron density of the entire molecule while neutrons are scattered by the atomic nuclei within the molecule.

\section{Small-Angle Scattering Method}

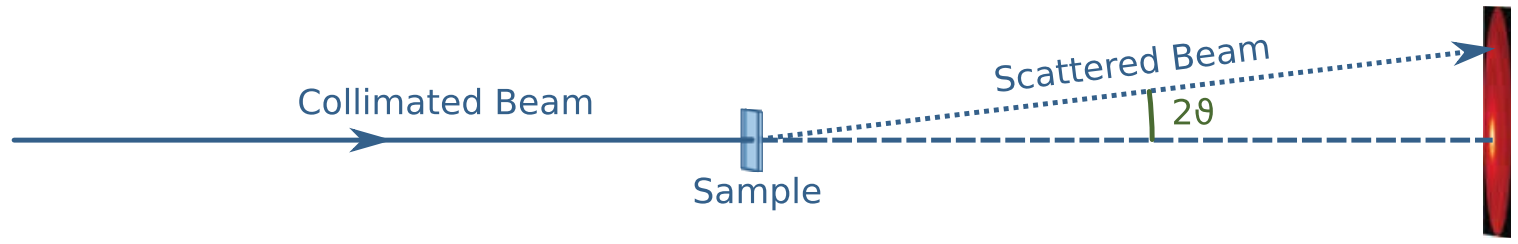

Figure 6. Small-Angle Scattering Setup: A beam of collimated radiation interacts with inhomogeneities in the sample matter. The elastic scattering from these interactions encode the temporal and spatial average of the distance distribution between atoms in the sample.

The method of SAS can be compared to optical microscopy. SAS and optical microscopy both investigate samples by shining a beam of incident radiation on a sample, then recording the scattered radiation, as illustrated in fig. 6. The primary difference for optical microscopy is the addition of a lens between the sample and the detector to reconstructs a real image of the matter. The recorded image would be a time average of that optical reconstruction over the recording time of the detector. ${ }^{3}$ For SAS, rather than using lenses to reconstruct a real image, the scattered radiation is directly recorded. The recorded image is still a time average for the duration of the exposure, but the image is a reciprocal space image rather than a real space image.

\footnotetext{
${ }^{2}$ One may conceive that added detail could be learned by increasing the energy to achieve an even smaller wavelength. This common idea that "more is always better" typically has its limits and this is true with biological SAXS. One must also consider how much energy the biological material can absorb before it begins to rapidly degrade, or cook.

${ }^{3}$ Using the $24 \mathrm{p}$ video standard, each frame is an average of $41.7 \mathrm{~ms}$.
} 
The recording process, by nature of the temporal averaging, fails to record the phase of the scattered radiation eliminating the possibility of recreating a 3D image of the scattering source. To process the recorded two-dimensional (2D) image, the scattering events from each pixel are binned according to the scattering angle, $2 \vartheta$, the details of which are discussed in Chapter 3. The resulting one-dimensional (1D) data contains the scattering intensity, $I(Q)$, as a function of the momentum transfer, $Q$, defined by the experimental setup using

$$
Q=4 \pi \frac{\sin (\vartheta)}{\lambda}
$$

\section{Structure Information From Small-Angle Scattering}

As a result of the temporal and spatial averaging during a SAS exposure, the measured $I(Q)$ represents the scattering from the ensemble of configurations explored by every molecule illuminated during the exposure. Mathematically, $I(Q)$ is the Fourier transform of the average pair distance distribution function, $P(r)$, of the illuminated sample, which gives the probability of finding two atoms separated by the distance $r$. For monodisperse solutions such that particle size and orientation are not correlated with position, this reciprocal space scattering intensity, $I(Q)$, can be written as the product of the single-particle form factor $P(Q)$ and the interparticle effective structure factor $S^{\prime}(Q)$,

$$
I(Q)=n_{p} P(Q) S^{\prime}(Q)
$$

where $n_{p}$ is the average number density of particles in the sample. We further can write

$$
S^{\prime}(Q)=1+\beta(Q)[S(Q)-1]
$$


where $\beta(Q)$ depends on the level of polydispersity and spherical symmetry of the particles, and $S(Q)$ is the actual interparticle structure factor (or the lattice factor for crystallography).

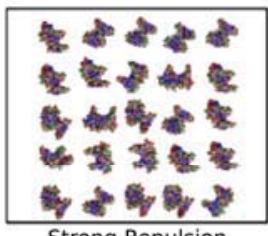

Strong Repulsion

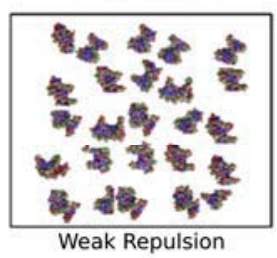

(a)
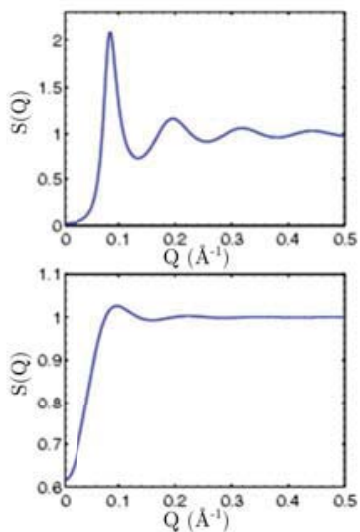

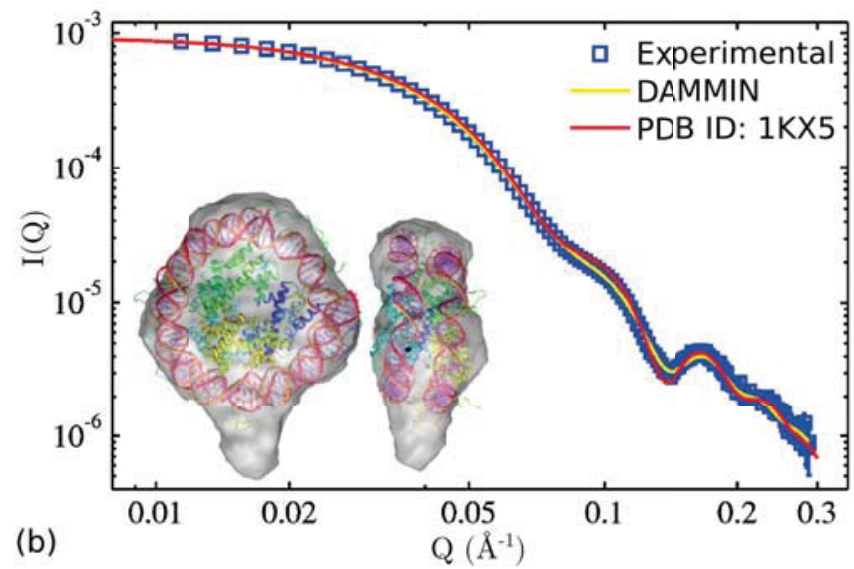

Figure 7. Scattering intensity is the product of the structure factor and the form factor: (a) The structure factor quantifies the order or disorder of a sample. As order increases, the scattering becomes more coherent with peaks corresponding to intermolecular spacing [66-68]. (b) The form factor encodes the distance distribution between the atoms in an individual particle. In a sufficiently dilute sample, the scattering intensity, $I(Q)$, and the form factor, $P(Q)$, are identical up to a constant scale factor. Here the experimental $I(Q)$ for a nucleosome is compared with the calculated theoretical scattering from the nucleosome crystal structure, PDB ID: 1KX5 [1], and from the theoretical mass envelope obtained from the experimental data using DAMMIN [69]. The inset shows the crystal structure overlain on the theoretical mass envelope obtained by iteratively comparing the calculated scattering from a volume of dummy atoms to the experimental form factor.

Features in $S(Q)$ arise from the non-random spatial distribution of biomolecules in solution. $S(Q)$ quantifies the order or disorder of molecules in solution as illustrated in fig. 7(a) comparing an ordered and disordered solution of the same molecules. The calculated $S(Q)$ curves show coherent peaks for the ordered solution, but these peaks are suppressed by the incoherent scattering from the disordered solution. Physically, the spatial distribution between molecules results from non-negligible intermolecular interactions which include a steric repulsion (caused by the finite sizes of biomolecules) 
and other molecule-specific interactions. In Chapter 2, we discuss using $S(Q)$ to quantify the interparticle pair potentials. For nucleosomes and nucleosome arrays, the interparticle potentials primarily depend on three factors, sample concentration, the length of the array (i.e., molecule size), and salt concentration (i.e., electrostatic screening).

The form factor is the direct Fourier transform of the pair distance distribution function of the scattering molecule, analogous to $I(Q)$ for the entire illuminated sample, and provides information on the size and shape of the individual molecules. Performing SAS measurements in a sufficiently dilute solution of identical molecules removes both interparticle interactions and polydispersity, $S^{\prime}(Q)=S(Q)=1$, and the scattering fully represents the average molecular structure, $I(Q)=n_{p} P(Q)$. As the scattering intensity scales linearly with the measurement time, $n_{p}$ is typically absorbed into an arbitrary scale factor leaving

$$
I(Q)=P(Q)
$$

The key to this process is to perform measurements below the concentration limit for interparticle interaction. Figure 7(b) compares just such a solution of nucleosomes to the theoretical scattering calculated from the nucleosome crystal structure [62].

This low concentration requirement is the primary reason we use SAXS rather than SANS for studying nucleosome structures. The key difference between SAXS and SANS is that X-ray beams have a much higher beam flux. As the flux of the beam on the sample increases, so too does the frequency of scattering interactions and thus an increased SNR. ${ }^{4}$ A high SNR is required to obtain high-quality data from nucleosome arrays as their size and charge set this low concentration limit beyond

\footnotetext{
${ }^{4}$ Again, "more is [not] always better" as increasing the beam flux also increases the rate of energy transfer to the sample. One must ensure that this transfer rate does not exceed the threshold of damaging the sample, or at least not within the time frame of the exposure. Liquid flow cells serve as a common solution to this complication.
} 
what is reasonable using SANS for the $Q$-range required.

Juxtaposing a SAXS setup with a SANS setup illustrates their complementary nature. For SAXS, a typical photon beam configuration at the Cornell High Energy Synchrotron Source (CHESS) G1 hutch radiates at $3 \times 10^{11}$ photons/s at a wavelength of $1.26 \AA$ [70]. A typical SANS beam from the nuclear reactor at the National Institute of Standards and Technology Center for Neutron Research, NG-3, radiates at $6.3 \times 10^{6}$ neutrons/s at a wavelength of $5 \AA$ [71]. The increase in flux by five orders of magnitude for the SAXS setup in this comparison produces a significantly increased SNR thereby allowing for measurements at much lower concentrations.

Once the SNR and concentration are such to obtain the $P(Q)$, the most basic values to extract from the scattering intensity are the scattering at zero angle, $I(Q=$ 0 ), and the radius of gyration, $R_{g}$. The $R_{g}$ of a particle about its center of mass is analytically written as [72]

$$
R_{g}^{2}=\frac{\int_{0}^{\infty} P(r) r^{2} d r}{2 \int_{0}^{\infty} P(r) d r}
$$

where $P(r)$ is the pair distance distribution function, describing the probability of atoms being separated by a distance $r$. The relation between $R_{g}$ and the scattering intensity can be derived by expanding the classical definition of scattering intensity $[72]$

$$
I(Q)=4 \pi \int_{0}^{\infty} P(r) \frac{\sin (Q r)}{Q r} d r
$$

using the Taylor series expansion of $\sin (Q r)$. Recalling that $Q \propto \sin (\vartheta)$, for small angles we perform this expansion about $Q=0$, to find,

$$
\sin (Q r)=Q r-\frac{(Q r)^{3}}{3 !}+\frac{(Q r)^{5}}{5 !}+\mathcal{O}\left((Q r)^{7}\right)
$$


Substituting eq. (8) into eq. (7) then simplifying we get,

$$
I(Q)=4 \pi\left[\int_{0}^{\infty} P(r) d r-\frac{Q^{2}}{3 !} \int_{0}^{\infty} P(r) r^{2} d r+\frac{Q^{4}}{5 !} \int_{0}^{\infty} P(r) r^{4} d r+\mathcal{O}\left((Q r)^{6}\right)\right]
$$

We further simplify this by factoring out the first term and, because $Q \ll 1$, only keep up to the second order in $Q$ giving,

$$
I(Q)=4 \pi \int_{0}^{\infty} P(r) d r\left[1-\frac{Q^{2}}{3 !} \frac{\int_{0}^{\infty} P(r) r^{2} d r}{\int_{0}^{\infty} P(r) d r}+\mathcal{O}\left((Q r)^{4}\right)\right]
$$

The zeroth order term is the definition of the scattering intensity at $Q=0$,

$$
I(0)=4 \pi \int_{0}^{\infty} P(r) d r
$$

and depends solely on the number of electrons in the sample scattering volume. Consequently, when data is put on an absolute scale, this provides a useful means of calculating the molecular weight of the scattering molecule.

Together eqs. (6), (10) and (11) combine to,

$$
I(Q)=I(0)\left[1-\frac{Q^{2}}{3} R_{g}^{2}+\mathcal{O}\left((Q r)^{4}\right)\right]
$$

Guinier recognized the region in brackets is a Taylor series expansion of $\exp (x)$ with $x=-Q^{2} R_{g}^{2} / 3$ which upon substitution gives the Guinier approximation [73]

$$
I(Q) \approx I(0) \exp \left(-Q^{2} R_{g}^{2} / 3\right)
$$

Taking the natural log of this,

$$
\log (I(Q)) \approx \frac{-R_{g}^{2}}{3} Q^{2}+\log (I(0))
$$


we see that when plotting the scattering data as $\log (I(Q))$ versus $Q^{2}$, referred to as a Guinier plot, $R_{g}^{2}$ is proportional to the initial slope (at lowest $Q$ ) $[72,74]$.

In addition to calculating $I(0)$ and $R_{g}$, one can calculate the reverse Fourier transform of $I(Q)$ to get $P(r)$, using [72],

$$
P(r)=\frac{1}{2 \pi^{2}} \int_{0}^{\infty} I(Q) \sin (Q r) Q r d Q
$$

Whereas $I(Q)$ is in momentum space, commonly referred to as reciprocal space, $P(r)$ is in real space and represents the probability of atoms being separated by a distance $r$. Equation (15) describes the ideal case, but in actuality we cannot measure $I(Q)$ for the full range $0 \leq Q \leq \infty$. We therefore approximate using,

$$
P(r) \approx \frac{1}{2 \pi^{2}} \int_{Q_{\min }}^{Q_{\max }} I(Q) \sin (Q r) Q r d Q
$$

with the added constraints that $P(0)=0$ and $P\left(r \geq D_{\max }\right)=0$, where $D_{\max }$ is the empirically determined maximum distance between atoms in the particle.

Beyond calculating the $I(0), R_{g}$, and $P(r)$, one can further extract structural information from scattering data using peak analysis and whole curve modeling. Peak analysis examines peaks in $I(Q)$ using the Bragg equation [72], which relates momentum transfer to molecular repeat distances,

$$
D_{\text {repeat }}=\frac{2 \pi}{Q}
$$

and the Scherrer equation [75], which relates peak width, $\sigma_{\text {peak }}$, to the coherent length of ordering,

$$
D_{\text {order }}=\frac{2 \pi}{\sigma_{\text {peak }}} .
$$


Equations (17) and (18) provide a straightforward mechanism for measuring quantities such as the packing parameters for DNA arrays [76], DNA wrapped carbon nanotubes [77], or DNA-DNA spacing within a virus capsid [78]. Whole curve modeling is a more complicated process which involves comparing the experimental $I(Q)$ to the theoretical $I(Q)$ calculated from model structures ranging in complexity from geometric shapes, such as spheroids, toroids, and cylinders, to complete atomistic structures. This is the modeling approach we take for analyzing nucleosome arrays, discussed in detail in Chapters 4 and 5 . 


\title{
Chapter 2: Structure and Interaction of Nucleosome Monomers
}

\section{Chapter Overview}

The first step in exploring the solution structure of nucleosome arrays is to gain a clear understanding of the structure and interaction of NCPs in solution. This understanding provides the foundation for surveying more complex arrays of nucleosome.

The following peer reviewed publication documents our study of natural-source NCPs (NS-NCP), and two NCP constructs with epigenetic modifications. By varying the NCP and ion concentrations, abbreviated as [NCP] and [ion], we quantify the inter-NCP pair potentials. We also compare the difference interaction effects caused by $\mathrm{H} 3$ versus H4 histone modifications.

\section{Elucidating Internucleosome Interactions and the Roles of Hi- stone Tails}

Steven C. Howell, ${ }^{\dagger}$ Kurt Andresen, ${ }^{\ddagger}$ Isabel Jimenez-Useche ${ }^{\S}$, Chongli Yuan, ${ }^{\S}$ and Xiangyun Qiu ${ }^{\dagger}$

†Department of Physics, George Washington University, Washington, DC;

${ }^{\ddagger}$ Department of Physics, Gettysburg College, Gettysburg, Pennsylvania;

$\S$ School of Chemical Engineering, Purdue University, West Lafayette, Indiana

Biophysical Journal Volume 105 July 2013 194-199

DOI: $10.1016 / j . b p j .2013 .05 .021$

\begin{abstract}
The nucleosome is the first level of genome organization and regulation in eukaryotes where negatively charged DNA is wrapped around largely positively charged histone proteins. Interaction between nucleosomes is dominated by electrostatics at long range and guided by specific contacts at short range, particularly involving their
\end{abstract}


flexible histone tails. We have thus quantified how internucleosome interactions are modulated by salts $\left(\mathrm{KCl}, \mathrm{MgCl}_{2}\right)$ and histone tail deletions (H3, H4 N-terminal), using SAXS and theoretical modeling. We found that measured effective charges at low salts are $\sim 1 / 5^{\text {th }}$ of the theoretically predicted renormalized charges and that H4 tail deletion suppresses the attraction at high salts to a larger extent than $\mathrm{H} 3$ tail deletion.

\section{Introduction}

Highly charged DNA is packaged into NCP in eukaryotic cells by wrapping every 147 per $\sim 180 \mathrm{bp}$ of DNA around a histone octamer. The octamer is a protein complex comprising two copies of each of the four histone proteins (named H2A, H2B, H3, and H4). Each histone has one C-terminal and one N-terminal tail that protrude out from the octameric core. Linked by 20-60 bp DNA like beads on a string, NCPs are further packaged into chromatin whose conformation and dynamics underpins gene maintenance and regulation. Thus, there has been long-standing interest in physically understanding nucleosome structure and interaction fundamental to chromatin function.

Polyelectrolyte behavior and histone tail modulation are among the key determinants of the multifactorial packaging of nucleosomes [38]. One NCP carries a net negative charge of $\sim 150 e$ with $-294 e$ from DNA and $+144 e$ from histone proteins. Given its diameter of $\sim 10 \mathrm{~nm}$ and height of $\sim 6 \mathrm{~nm}$, this leads to an average charge density of $-0.43 \mathrm{e} / \mathrm{nm}^{2}$, about half of the $-1 \mathrm{e} / \mathrm{nm}^{2}$ of DNA. The expected polyelectrolyte behavior of NCP is demonstrated by cation-modulated unfolding and compaction of NCP arrays [35, 79]. Notably, divalent cations go beyond screening the electrostatic repulsion between NCPs and can mediate inter-NCP attraction, e.g., $\mathrm{Mg}^{2+}$ or $\mathrm{Ca}^{2+}$ induced condensation of mono- and poly-nucleosomes [41]. In contrast, these divalent cations are unable to condense DNA. This suggests significant roles of 
the complex charge distribution of NCP furnished by the histones [43]. In particular, the tails of histones ( $\sim 30 \%$ of the histone mass), being highly positively charged and flexible, are known to be crucial in modulating nucleosome interaction and chromatin assembly [38].

There have been extensive experimental studies aiming to elucidate the electrostatics of nucleosomes and the roles of histone tails. The effects of cations of varied valences on the nucleosome assembly have been well characterized [35, 38, 39, 79], e.g., by measuring the sedimentation coefficients of nucleosome arrays or the fraction of condensed nucleosomes $[40,41]$. Among the 16 histone tails, the eight N-terminal tails hold the most positive charges and are considered more significant, e.g., NCPs with all N-terminal tails deleted remain soluble in high divalent salts [50]. In an effort to dissect the contribution from each N-terminal tail, all 16 possible tail-deletion NCP mutants have been studied and it was found that all N-terminal tails contribute to the condensation of oligo-NCP arrays nonspecifically and additively [50]. Still, the histone tails vary in length and charge and thus differ in strength in mediating nucleosome interactions, e.g., H3 and H4 tails, studied herein, were observed to be more important than $\mathrm{H} 2 \mathrm{~A}$ and $\mathrm{H} 2 \mathrm{~B}$ tails $[50,52]$.

Systematic measurements have promoted widespread theoretical interests in mechanistically understanding the structure and interaction of nucleosomes [5, 80]. At the atomic level, enabled by the availability of high resolution crystal structures [81], molecular dynamics simulations have described the ion and solvent atmospheres of nucleosome and revealed rugged electrostatic surfaces [82, 83]. The flexible histone tails have been analyzed in detail and shown to display multistate conformational dynamics [84-86]. At the CG level, both nucleosome arrays and chromatin have been modeled to recapture the conformational changes under varied conditions such as salt and mechanical stretching $[59,87]$. However, to apply the detailed NCP structures and energetics predicted by theoretical approaches, stringent experimental tests 
are required, but largely lacking. For example, as the focus of this study, one key factor to the assembly of NCPs is the effective inter-NCP potential that is usually computed following the Poisson-Boltzmann formalism for efficiency [80]. However, such theoretical treatment has not been validated experimentally due to the lack of direct measurements of inter-NCP potentials. Comparisons with previously measured quantities are often difficult, e.g., the interpretation of sedimentation coefficient necessitates an ab initio hydrodynamic model.

Here, we report measurements of interactions between monomeric nucleosomes using solution SAXS in conjunction with theories of polyelectrolytes. There are two outcomes we deem important: 1), Quantification of inter-NCP pair potentials by modeling full SAXS profiles; 2), Elucidation of the individual role of $\mathrm{H} 3$ and $\mathrm{H} 4$ tails. Although SAXS (and small-angle neutron scattering for neutron) has been used to study the structure and interaction of NCPs since the 1970s [88], no quantitative refinement of full SAXS profiles has been done to extract the inter-NCP interactions, which we obtain here by refining synchrotron SAXS data. One key advantage of full profile modeling is quantification of the inter-NCP potential as a function of inter-NCP distance [89], in contrast to single-parameter measurements such as second virial coefficients or osmotic pressures of NCP solutions. Bertin et al. [90] have compared full SAXS profiles with theoretical calculations. However, refinements were not performed, possibly due to the relatively dilute NCP concentrations $(\leq 8 \mathrm{mg} / \mathrm{mL}$, $\sim 0.04 \mathrm{mM}$ ) and limited angular range of the in-house SAXS data that lower SAXS sensitivity to inter-NCP interactions. In addition, using recombinant NCP constructs with either $\mathrm{H} 3$ or $\mathrm{H} 4$ tail deleted, to the best of our knowledge we report the first analysis of this kind on the respective role of $\mathrm{H} 3$ and $\mathrm{H} 4$ tails in mediating inter-NCP interactions. 


\section{Material and Methods}

Monomeric NS-NCPs were prepared from adult chicken erythrocytes as in [91]. Gel electrophoresis was used to assess the DNA length (147 $\pm 5 \mathrm{bp})$ and the histone content (fig. 67, (i) and (ii), in appendix A). Recombinant nucleosomes (RC-NCP) were reconstituted by mixing $147 \mathrm{bp}$ mouse mammary tumor virus (MMTV) DNA and histone octamers with sequences from Xenopus laevis following standard protocols [50] (fig. 67, (i) and (iii)). The globular H3 and H4 recombinant proteins were truncated at their N-terminal ends (referred to as gH3 and gH4 RC-NCPs), by deleting the coding sequences of amino acids (1-27 with $+10 e$ of $\mathrm{H} 3$ and 1-10 with $+3 e$ of H4) via site-directed mutagenesis. All NCP stocks were purified by size-exclusion chromatography (Sephacryl S300-HR medium) and then dialyzed against $10 \mathrm{mM} \mathrm{KCl}$ $10 \mathrm{mM}$ Tris $0.1 \mathrm{mM}$ EDTA pH 7.5 buffer. Salts were adjusted to studied conditions by adding concentrated salts. SAXS experiments were carried out at $20^{\circ} \mathrm{C}$ at the G1 station at the Cornell High Energy Synchrotron Source in Ithaca, New York. The incident beam had an energy of $9.97 \mathrm{keV}$ and size of $250 \times 250 \mu \mathrm{m}^{2}$. Samples of $\sim 30 \mu \mathrm{L}$ were injected into an in-vacuum capillary flow cell to enable windowless data collection for background reduction. Radiation damage was mitigated by optimizing X-ray exposure time and oscillating the plug of sample solution. Six to eight two-second exposures of the same sample were taken and verified to be reproducible, indicative of the absence of radiation damage.

\section{Results}

Measured SAXS profile $I(Q)$ is a product of the form factor $P(Q)$ and the structure factor $S(Q)$, i.e., $I(Q)=P(Q) \times S(Q)$, with $S(Q)$ becoming significant under conditions such as high $[\mathrm{NCP}] \mathrm{s}$ and strong inter-NCP interactions. $I(Q)$ of a dilute NCP solution (i.e., $S(Q)=1$ ) thus measures the structure of individual NCPs in terms of the form factor $P(Q)=I(Q)$. This can reveal differences between molecular struc- 
tures in solution and in crystal. Fig. 8 shows excellent agreement between the SAXS profile measured in solution and the profile calculated from a crystal structure in $Q$ range of $0.01-0.28 \AA^{-1}$, indicative of high sample purity and structural conformity. The same level of agreement was observed for all NCP constructs (i.e., NS-NCP, wildtype, gH3, and gH4 RC-NCPs). It is worth noting that the crystal structure $P(Q)$ shows an extra dip around $Q$ of $0.14 \AA^{-1}$. This discrepancy has been observed in previous reports $[90,92]$ and was attributed to the DNA unwrapping at the ends by a few base pairs. Tail deletions lead to small changes of the dip around $Q=0.14 \AA^{-1}$ that can be attributed to increased DNA unwrapping (data not shown). Nonetheless, these dips appear in relatively high $Q$ and do not affect our subsequent analysis. Guinier analysis of the low $Q$ data (fig. 8, inset) gives the radius of gyration $R_{g}=$ 44.4(3) $\AA$, which is nearly identical to previous SAXS measurements of natural-source NCPs [93] and recombinant NCPs with $\alpha$-satellite DNA [92]. Note that NCPs recon-

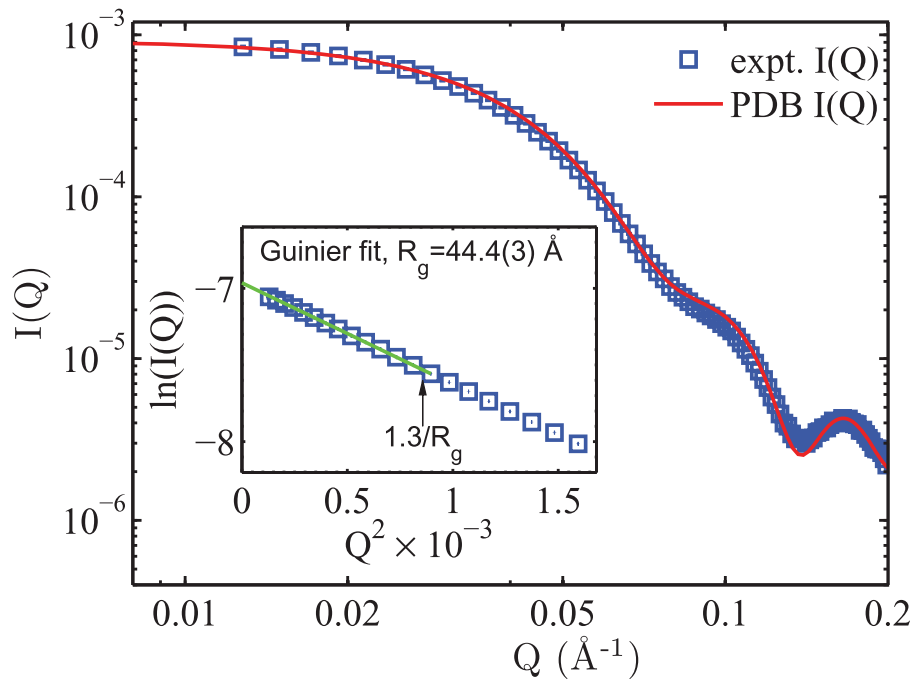

Figure 8. SAXS Form Factor: $P(Q)$ of NS-NCP from experiment ( $\square$ ) and crystal structure (red, PDB ID: 1KX5). The experiment form factor was measured at a dilute $\mathrm{NCP}$ concentration of $0.003 \mathrm{mM}(\sim 0.6 \mathrm{mg} / \mathrm{mL})$ and an intermediate salt $(40 \mathrm{mM} \mathrm{KCl})$ to minimize the influence of inter-NCP interferences. Error bars of $I(Q) \mathrm{s}$, in this and subsequent plots, are smaller than symbol sizes except at high $Q \mathrm{~s}$. Inset shows the Guinier fit (solid line) to obtain the radius of gyration $\left(R_{g}\right)$. The linear region extends well over the empirical $Q_{\max }$ cutoff of $1.3 / R_{g}$ for globular structures. 
stituted with the 601 sequence were reported to give slightly smaller $R_{g}$ by $2-3 \AA$ [90, $92]$.

In addition to structure information, SAXS is capable of quantifying intermolecular interactions, via the structure factor $S(Q)$ originating from spatial correlations between molecules. Qualitatively, repulsive interaction leads to a decrease of low $Q$ intensities, i.e., a downturn, and attractive interaction gives rise to an increase at

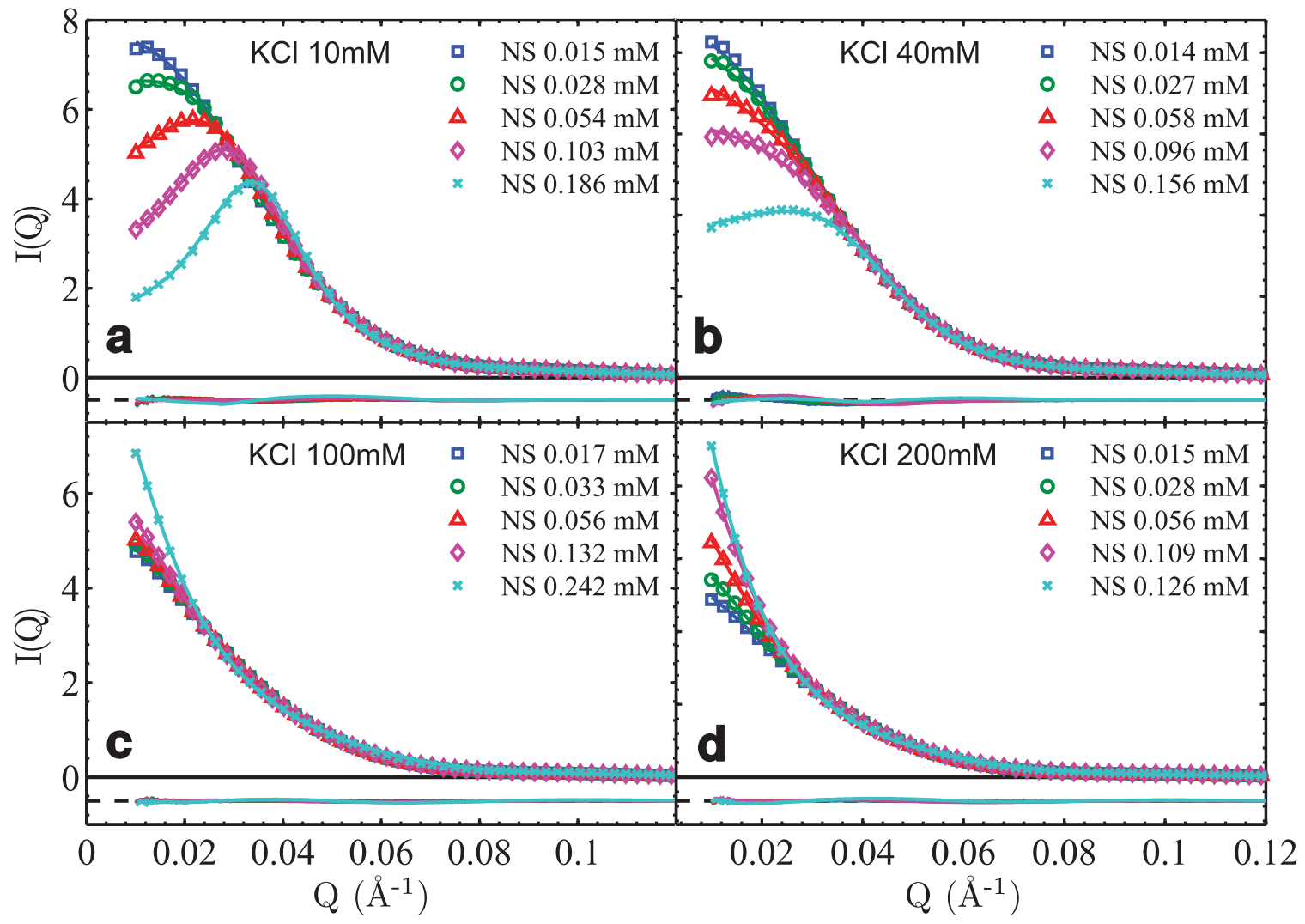

Figure 9. SAXS Profiles, $I(Q)=P(Q) \times S(Q)$, of NS-NCPs at Four $\mathrm{KCl}$ Concentrations: $\mathrm{I}(\mathrm{Q}) \mathrm{s}$ are normalized by NCP concentrations to assist comparison. Each panel shows experimental I(Q)s (symbols) at a series of $[\mathrm{NCP}] \mathrm{s}(0.1 \mathrm{mM}$ gives $\sim 20 \mathrm{mg} / \mathrm{mL}$ ) as indicated in the legends, together with their respective theoretical fits (lines). The residues are shown with an offset at the same scale. The pertinent pair-potential parameters are (discussed in detail in the main text), a: $Z_{\text {eff }}=22(1) e$, $\sigma_{N}=140 \AA ; \mathbf{b}: Z_{\text {eff }}=23(1) e, \sigma_{N}=110 \AA ; \mathbf{c}: Z_{\text {attr }}=150(5) e, \sigma_{N}=100 \AA ; \mathbf{d}:$ $Z_{\text {attr }}=200(8) e, \sigma_{N}=100 \AA$. Uncertainties of $\mathrm{Z}$ values originate from both curve fitting (via variance-covariance matrix, $\sim 1 \%)$ and small variations $(\sim 3 \%)$ between values at different $[\mathrm{NCP}] \mathrm{s}$. The $Z_{\text {eff }}$ and $Z_{\text {attr }}$ values are poorly defined when $[\mathrm{NCP}]$ is small $(<0.03 \mathrm{mM}$, or $6 \mathrm{mg} / \mathrm{mL})$ and their values given are from data at high $[\mathrm{NCP}] \mathrm{s}$. 
low Q, i.e., an upturn. Fig. 9 shows how SAXS profiles evolve with NCP and salt concentrations. As expected from strong inter-NCP repulsion at low salts (10 and $40 \mathrm{mM} \mathrm{KCl}$, fig. 9, a and b), low $Q$ downturns are observed and deepen upon increasing $[\mathrm{NCP}]$. In contrast, low $Q$ upturns appear and heighten with $[\mathrm{NCP}]$ at high salts (100 and $200 \mathrm{mM} \mathrm{KCl,} \mathrm{fig.} \mathrm{9,} \mathrm{(c)} \mathrm{and} \mathrm{(d)),} \mathrm{indicating} \mathrm{inter-NCP} \mathrm{attractions.} \mathrm{These}$ observations are consistent with reports of negative virial coefficients at $>70 \mathrm{mM}$ $\mathrm{KCl}$ [90]. Divalent $\mathrm{Mg}^{2+}$, known to condense NCP at $>2.5 \mathrm{mM}\left[\mathrm{Mg}^{2+}\right]$ [41], was also studied and the onset of inter-NCP attraction was found to be between 1 and $2 \mathrm{mM}$ [Mg $\left.{ }^{2+}\right]$ (fig. 69).

To gain quantitative insights into the underlying inter-NCP interactions, we then applied the generalized one component method (GOCM) with the mean spherical approximation $[89,94]$ to model the structure factors. As both repulsion and attraction exist, the general form of inter-NCP interaction potential as a function of inter-NCP distance is given by

$$
U(r)=\left\{\begin{array}{ll}
\infty & r<\sigma_{N} \\
\frac{Z_{\mathrm{eff}}^{2}}{\epsilon\left(1+\frac{\kappa_{D} \sigma_{N}}{2}\right)^{2}} \frac{e^{-\kappa_{D}\left(r-\sigma_{N}\right)}}{r}-\frac{Z_{\mathrm{attr}}^{2}}{\epsilon\left(1+\frac{\kappa_{\mathrm{attr}} \sigma_{N}}{2}\right)^{2}} \frac{e^{-\kappa_{\mathrm{attr}}\left(r-\sigma_{N}\right)}}{r} & r \geq \sigma_{N}
\end{array},\right.
$$

where $\sigma_{N}$ is the equivalent NCP diameter, $Z_{\text {eff }}$ and $Z_{\text {attr }}$ are the effective charges to be fitted, $\epsilon=80.4$ is the dielectric constant of water at $20^{\circ} \mathrm{C}$, and $\kappa_{D}$ is the inverse Debye screening length calculated from the salt condition. Namely, the inter-NCP potential comprises a hard-sphere (HS) repulsive term, a Debye-Hückel (DH) electrostatic repulsive term, and a DH-like attractive term. Although it is possible to include both DH potentials in the fitting procedures, there exist significant correlations between the fitted repulsive and attractive DH terms despite better fits (see appendix A for detailed results and discussions). Due to this complication, the attractive DH 
Table 2. Inter-NCP Interaction Parameters: Results from structure factor fittings with either one-DH potential (in the main text) or two-DH potential (in appendix A). As the structure factors are more pronounced at high NCP concentrations, the values in this table are taken as the mean over the 2-3 most concentrated samples for which the fits are the most reliable. For the fits with one DH term in the inter-NCP potential, the fitted effective charge $\left(Z_{\text {eff }}\right.$ or $\left.Z_{\text {attr }}\right)$ shows no systematic trends with the NCP concentration. For the fits with two DH terms, the fitted effective charge $\left(Z_{\text {eff }}\right)$ generally increases slightly with the increase of the NCP concentration. We do not know the reason for the different trends from fitting the same data and speculate that this may arise from how the generalized one component method handles the effects of sample concentration with or without an attractive term [89]. Note the values for the gH4-NCP construct at $40 \mathrm{mM} \mathrm{KCl}$ are unreliable due to the relative low $[\mathrm{NCP}] \mathrm{s}$.

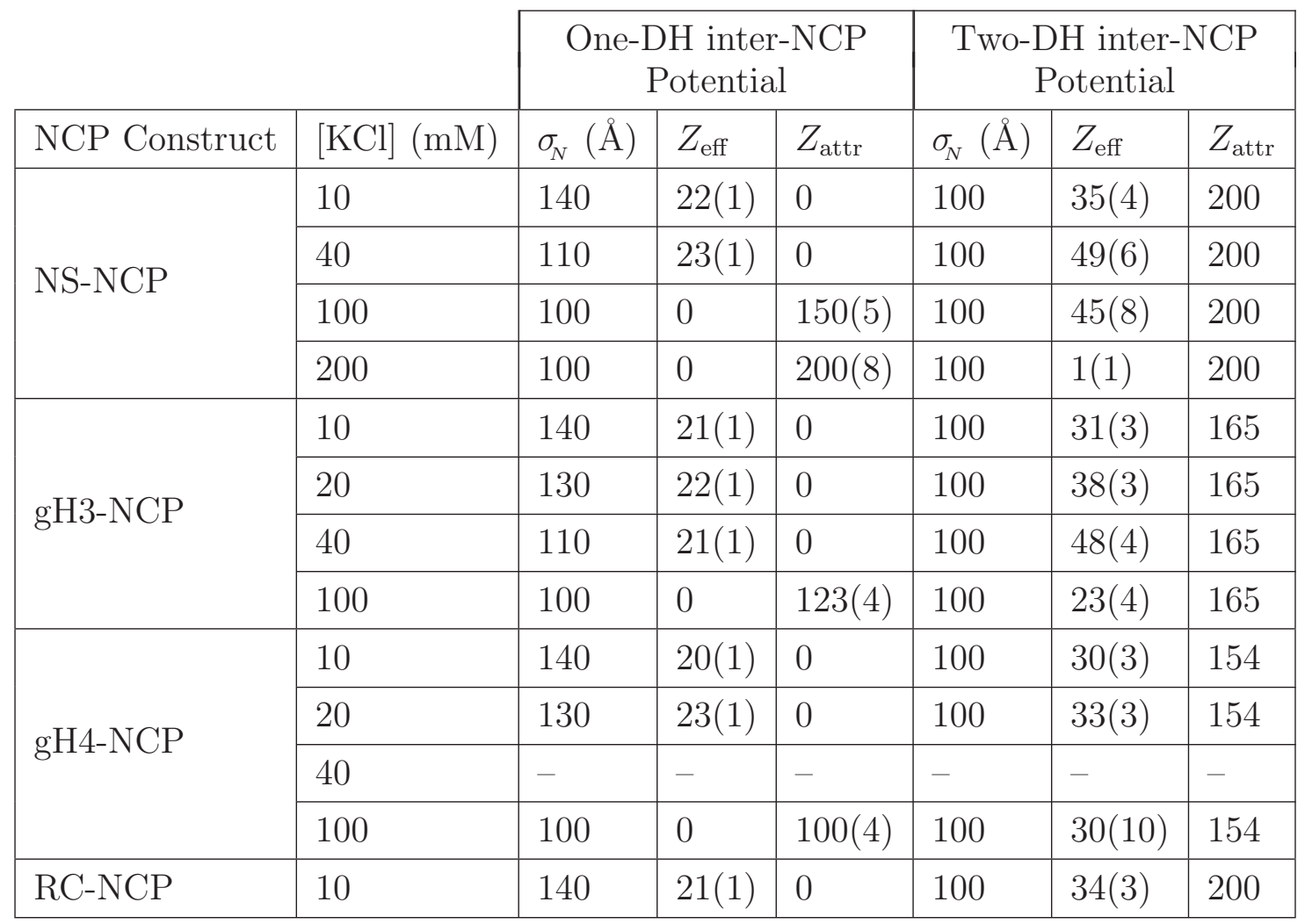

potential is turned off (i.e., $Z_{\mathrm{attr}}=0$ ) under conditions where a net inter-NCP repulsion is observed. Likewise, $Z_{\text {eff }}$ is set to 0 under conditions where a net attraction is observed. As the physical origin of the attraction is poorly understood, we chose a short-range attraction of effective charge $Z_{\text {attr }}$ and $4.8 \AA$ decay length for all data. The effect of NCP's disk-like shape (axis ratio $\sim 2$ ) on $S(Q)$ only becomes significant at $Q>0.036 \AA^{-1}[90]$ where $S(Q) \sim 1$ as observed in the experiments; it is thus not 
considered. Such GOCM fits are shown in fig. 9 as solid lines, noting that the form factor for each NCP construct is calculated based on the crystal structure (shown in fig. 68). It is evident that the GOCM fits are in good agreement with experimental data over the entire $Q$ range. Inter-NCP interactions are thus quantified in the forms of HS and DH potentials.

Despite being quantitative, directly usable, and comparable with theoretical studies, it should be noted that the sum of HS and DH potentials describes an apparent inter-NCP interaction that reproduces the measured structure factor. In the case of inter-NCP repulsion, the effective charge $Z_{\text {eff }}$, as the only fitting parameter, differs from the bare charge $Z_{\text {bare }}$ due to the nonlinear nature of ion screening and the validity of DH potentials in linear regime only. For the same reason, the equivalent diameter $\sigma_{N}$ encloses the vicinity with a large electrostatic field (e.g., $>3.8 k_{B} T / e$ was used for dsDNA by the authors [68]), which is in the order of Debye length. Especially at low salt, $\sigma_{N}$ is significantly larger than the $100 \AA$ computed from the NCP crystal structure [89]. We determined the value of $\sigma_{N}$ at different salts based on cell model numerical calculations $[68,95]$, and the same $\sigma_{N}$ value was then fixed for all data at the same ionic condition for consistency. We show these relevant interaction potential parameters in the figure captions and in table 2 .

Figure 10 shows data from recombinant gH3 and gH4 RC-NCPs at two salt conditions (10 and $100 \mathrm{mM} \mathrm{KCl)} \mathrm{together} \mathrm{with} \mathrm{GOCM} \mathrm{fits} \mathrm{(data} \mathrm{at} 20$ and $40 \mathrm{mM} \mathrm{KCl}$ are shown in fig. 70). We primarily focused on gH3 and gH4 RC-NCPs because recombinant samples were relatively limited in volume and previous studies have reported the second virial coefficients (though no inter-NCP potentials) of intact RC-NCPs and gH3gH4 RC-NCPs, i.e., with none or both tails deleted [90]. To enable crosscomparisons, intact RC-NCPs were measured at a few selected conditions (fig. 71). At low salt of $10 \mathrm{mM} \mathrm{KCl}$, essentially no difference in the effective charge $Z_{\text {eff }}(\sim 22 e)$ was observed among all NCP constructs (i.e., NS-NCP, gH3, gH4, and intact RC-NCPs), 
noting that the negativity of $Z_{\text {eff }}$ is omitted for brevity. We further calculated the second virial coefficient from the inter-NCP potential using

$$
A_{2}=\frac{1}{2} \int_{0}^{\infty}\left(1-e^{U(r) / k_{B} T}\right) 4 \pi r^{2} d r
$$

and obtained a value of $2.5(1) \times 10^{-4} \mathrm{~mL} \cdot \mathrm{mol} / \mathrm{g}^{2}$. This value is in excellent agreement with the osmometry measurement by Mangenot et al. [96]. Bertin et al. [90] used theoretical calculations of $Z_{\text {eff }}$ between 70 and $125 e$ and obtained much larger $\mathrm{A}_{2}$

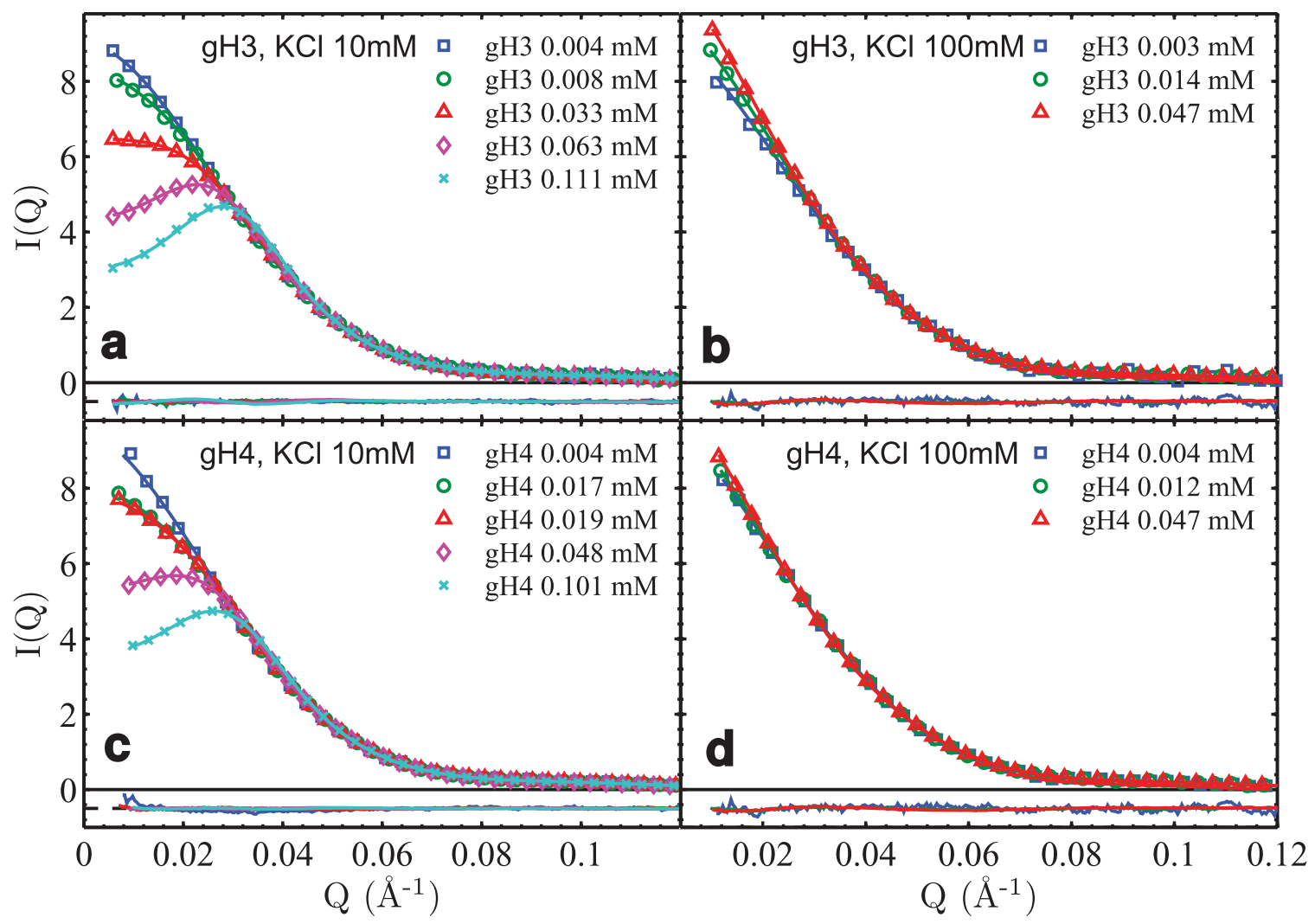

Figure 10. SAXS Profiles, $I(Q)=P(Q) \times S(Q)$, of gH3 and gH4 RC-NCPs at 10 and $100 \mathrm{mM} \mathrm{KCl:} \mathrm{Annotations} \mathrm{follow} \mathrm{that} \mathrm{of} \mathrm{fig.} \mathrm{9.} \mathrm{Inter-NCP} \mathrm{potential}$ parameters are, a: $Z_{\text {eff }}=21(1) e, \sigma_{N}=140 \AA ; \mathbf{b}: Z_{\text {attr }}=123(4) e, \sigma_{N}=100 \AA$; c: $Z_{\text {eff }}=20(1) e, \sigma_{N}=140 \AA ; \mathbf{d}: Z_{\text {attr }}=100(4) e, \sigma_{N}=100 \AA$. It is worth noting that, although gH4 RC-NCP shows no interaction at $100 \mathrm{mM} \mathrm{KCl}$, a significant $Z_{\text {attr }}$ exists so as to negate the HS repulsion with $\sigma_{N}=100 \AA$, which makes $Z_{\text {attr }}$ values artificially large. $Z_{\text {attr }}$ would be zero if $\sigma_{N}$ is zero for gH4 RC-NCP in $100 \mathrm{mM} \mathrm{KCl}$. It is thus more appropriate to interpret $Z_{\text {attr }}=100$ as contributed by the inter-NCP short-range attraction. 
values close to $5.0 \times 10^{-4} \mathrm{~mL} \cdot \mathrm{mol} / \mathrm{g}^{2}$. We attribute the difference to the discrepancy between theoretical and experimental $Z_{\text {eff }}$ values (discussed below) used by Bertin et al. and us, respectively. At high salt of $100 \mathrm{mM} \mathrm{KCl}$, qualitative differences were observed: NS-NCP, gH3 and intact RC-NCPs show comparable levels of attraction; whereas gH4 and gH3gH4 RC-NCPs show no attraction (including observations in [90]). This suggests the major role of H4 tail in mediating inter-NCP attractions.

\section{Discussion}

We consider the quantification of inter-NCP pair potentials from SAXS structure factors as the most interesting result of our study. Compared with the traditional analysis of second virial coefficients that relies on $I(Q=0)$ only, the GOCM (and methods alike) uses the full SAXS profiles and obtains self-consistent pair potentials allowing quantitative comparisons with theoretical predictions. At low salts such as $10 \mathrm{mM} \mathrm{KCl,} \mathrm{NCP-NCP}$ interactions are dominated by repulsion as expected. Essentially the same $Z_{\text {eff }}$ is obtained for all four NCP constructs. The minimal effect of tail deletions on inter-NCP repulsion is not surprising given that tail deletions change NCP bare charges by $<8 \%$ and that cations can effectively fill in for the deleted monocharged amino acids. Quantitatively, it is instructive to compare the measured $Z_{\text {eff }}$ with the theoretical renormalized charge $Z_{\text {ren }}$ that can be calculated following the charge renormalization theorem [95]. For dsDNA with negatively charged groups only, our previous work showed that the measured $Z_{\text {eff }}$ is fairly consistent (within 10-25\%) with its renormalized charge $Z_{\text {ren }}$ in monovalent salts [68]. However, for the nucleosome, its $Z_{\text {eff }}(\sim 22 e)$ is far smaller than theoretical $Z_{\text {ren }}$ (> $100 e$ for all experimental conditions), noting that $Z_{\text {bare }}$ is $\sim 150 e$ and $Z_{\text {ren }}$ weakly depends on NCP and salt concentrations [95]. Given that the $Z_{\text {ren }}$ is calculated by treating NCP as a uniformly charged sphere, such drastic and physically significant discrepancy is likely due to the charge nonuniformity of NCP in terms of spatial distribution and 
sign, resulting in highly anisotropic behaviors where the uniformly charged surface consideration breaks down.

Inter-NCP interactions at short range are further complicated by the flexible histone tails $[80,85]$. At elevated salts where electrostatic repulsion is greatly weakened, histone tail deletions can significantly affect NCP-NCP interactions. At $100 \mathrm{mM} \mathrm{KCl}$ (fig. 9 c, fig. 10 b and d), NS-NCP shows significant attraction (i.e., low $Q$ upturn), gH3 RC-NCP shows attraction of comparable magnitude, and gH4 RC-NCP shows nearly no attraction. The difference between gH3 and gH4 RC-NCPs, which have not been studied by SAXS previously, suggests that the $\mathrm{H} 4$ tail is more important in mediating NCP-NCP attractions than the $\mathrm{H} 3$ tail. This corresponds well with the observed most important role of the $\mathrm{H} 4$ tail in assembling the 30-nm chromatin fiber [40]. Such trend is also consistent with the repulsion between gH3gH4 RC-NCPs up to $200 \mathrm{mM} \mathrm{KCl} \mathrm{[90].} \mathrm{The} \mathrm{more} \mathrm{important} \mathrm{role} \mathrm{of} \mathrm{the} \mathrm{H} 4$ tail in mediating internucleosome interactions has been suggested by Arya and Schlick [97] analyzing a CG mesoscale structure model with a tailored configurational-bias Monte Carlo method. As $\mathrm{H} 4$ tail deletion removes fewer charges than $\mathrm{H} 3$ tail deletion $(+3$ versus $+10 e)$ but shows a larger effect, this alludes to specific interactions of $\mathrm{H} 4$ tails with adjacent NCPs in close approach. To probe the likely H4-tail-DNA interactions, Korolev and Nordenskiöld [98] performed all-atomic molecular dynamics simulations of DNA and H4 tails and suggested possible lysine-mediated bridging and minor groove dynamic binding. Another possible explanation is $\mathrm{H} 4$-tail binding with the $\mathrm{H} 2 \mathrm{~A} / \mathrm{H} 2 \mathrm{~B}$ anionic patch on neighboring NCPs, as suggested by crystallography studies [62, 99]. This is in agreement with recent experimental studies of the effects of $\mathrm{H} 4$ tail lysine acetylation on nucleosome array folding that proposed site-specific lysine binding to the $\mathrm{H} 2 \mathrm{~B}$ histone [52]. To fully establish the underlying molecular mechanisms, experimental approaches probing the local structure would be needed to resolve the physical origins of inter-NCP short range attraction. 


\section{Conclusion}

In conclusion, we have measured repulsive and attractive NCP-NCP interactions under various salt conditions with distinct NCP constructs. Quantitative knowledge of inter-NCP pair potentials should aid theoretical studies of nucleosome and chromatin high-order structure and dynamics. The physical understanding to be gained will shed light on the broad class of multiphasic macromolecules with two or more different interacting groups. Work is underway to relate the inter-NCP interactions to the conformation and energetics of nucleosome arrays through measurement and modeling.

\section{Chapter Summary}

This initial study of single nucleosome structure and interaction establishes the provides an understanding of the internucleosome interactions underpinning the more complicated study of nucleosome arrays. The agreement between the NS-NCP form factor and the calculated $1 \mathrm{KX} 5$ crystal NCP [1] form factor indicates that it is reasonable to expect the nucleosome structure within the array to be relatively unchanged under the same ionic conditions. This process of comparing the experimental and calculated form factors introduces the concept of matching atomistic structures to SAXS results, a fundamental process for analyzing the nucleosome arrays. Recognizing that the electrostatic interactions of nucleosomes are a small fraction of the theoretically renormalized charge provides evidence that nucleosomes within an array may not repel each other as much as would be expected in the case of a uniform charge distribution. Lastly, the experience gained in these preliminary studies was crucial to the becoming familiar with the measurement and analysis process to be discussed further in the following chapter. 


\section{Chapter 3: SAXS Experiments of Nucleosome Arrays}

\section{Chapter Overview}

In order to explore the conformation and dynamics of short nucleosome arrays, we measured well-defined recombinant nucleosome constructs in vitro using solution SAXS. We assembled nucleosome arrays using Xenopus laevis histone protein octamers [44] and recombinant DNA. We designed the DNA specifically with $N$ repeats of the 147 bp Widom 601 [4] (W601) sequence known for its high affinity for histone octamers. By using the W601 positioning sequence connected by 20 bp DNA linkers, arrays have a consistent distance and relative twist between adjacent nucleosomes thereby reducing the system complexity. Our primary interest was to compare the salt-induced conformational changes in arrays containing 2, 3, 4, and 12 NCPs, referred to as $N \times 167$, where $N$ is the number of NCP repeats. We also considered the effect of added linker histone gH5 on the solution structure of the $4 \times 167$ array.

We report here the scattering results from these nucleosome arrays in the presence of various concentrations of $\mathrm{KCl}$ and $\mathrm{MgCl}_{2}$. During one set of measurements, we examined $3 \times 167$, and $4 \times 167$ arrays in 10,50 , and $100 \mathrm{mM} \mathrm{KCl}$ as well as $1 \mathrm{mM} \mathrm{MgCl}_{2}$ and also measured $2 \times 167$ arrays in $10 \mathrm{mM} \mathrm{KCl}$. In another set of measurements, we examined a second set of $3 \times 167$ arrays in 10, 50, 100, and $200 \mathrm{mM} \mathrm{KCl}$ and also investigated the effects of linker histone by comparing measurements of $4 \times 167$ and $12 \times 167$ arrays constructs with and without $\mathrm{gH} 5$ protein in $10 \mathrm{mM} \mathrm{KCl}$ and $1 \mathrm{mM}$ $\mathrm{MgCl}_{2}$. The form factors from all arrays constructs show a consistent salt-induced compaction as increased cation concentration screens the repulsion of the negatively charged nucleosomes. By virtue of this cation screening, we report the ionic buffer conditions based on the cation concentration, e.g., $10 \mathrm{mM} \mathrm{K}{ }^{+}$. We also point out

that all $\mathrm{MgCl}_{2}$ solutions also contain $10 \mathrm{mM} \mathrm{KCl}$ but we only refer to the $\mathrm{Mg}^{2+}$ concentration for brevity. 
To identify differences between the solution and crystal structures, we compare the form factors of all $4 \times 167$ arrays to the previously reported crystal structure of a similar 4×167 nucleosome array, PDB ID: 1ZBB [2]. This comparison showed close agreement between the $1 \mathrm{ZBB}$ structure and the $4 \times 167$ array in $1 \mathrm{mM} \mathrm{Mg}^{2+}$, which agreed with previous reports that multivalent cations and linker histones produce maximum compaction of chromatin in vitro [40, 99]. To better understand the differences between the compact $4 \times 167$ array in $1 \mathrm{mM} \mathrm{Mg}^{2+}$ and the crystal structure, we performed manual rigid body modeling based on the 1ZBB structure and found the best-matched models by slightly altering the stacking of the NCPs.

\section{Sample Preparation}

\section{Protein Expression and Purification}

Each histone (H2A, H2B, H3, and H4) was expressed in the BL21(DE3)pLysS cells of Escherichia coli (E. coli). The cell culture was grown in lysogeny broth (LB) medium containing $100 \mathrm{mg} / \mathrm{mL}$ ampicillin and $25 \mathrm{mg} / \mathrm{mL}$ chloramphenicol at $37^{\circ} \mathrm{C}$ under vigorous stirring, 225 revolutions per minute (rpm), and induced with $0.4 \mathrm{mM}$ isopropyl-1- $\beta$-Dthiogalactoside when the optical density at $600 \mathrm{~nm}$ reached $0.4-0.6$. Protein expression continued for another 3 hours at $37^{\circ} \mathrm{C}$. The cells were harvested by centrifugation and resuspended in precooled

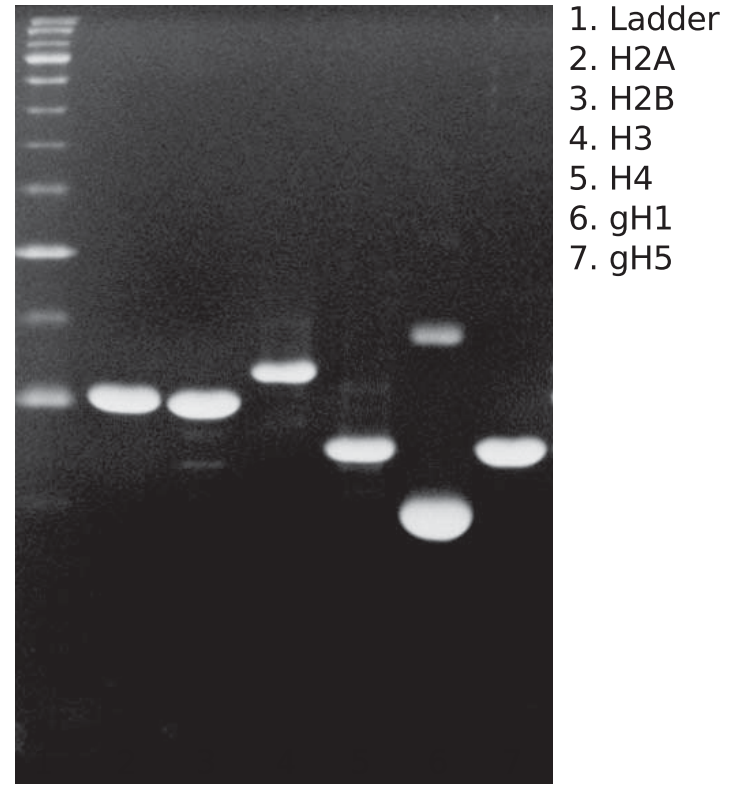

Figure 11. SDS-PAGE Gel of the Histone Proteins: The proteins for the NCP core, H2A, H2B, H3, and H4, show a high level of purity, indicated by the single strong band in each lane. The gH5 proteins are also pure. The faint band in the globular linker histones H1 (gH1) lane indicates contamination. Consequently we exclude measurements from gH1 arrays. 
wash buffer ( $50 \mathrm{mM}$ Tris-HCl, pH 7.5, $100 \mathrm{mM} \mathrm{NaCl}, 1 \mathrm{mM}$ Na-EDTA, $0.2 \mathrm{mg} / \mathrm{mL}$ PMSF), and sonicated on ice for 10 minutes (min). Next, the cell lysate was centrifuged at $12000 \mathrm{rpm}$ for $30 \mathrm{~min}$ at $4{ }^{\circ} \mathrm{C}$. Subsequently, the inclusion bodies were prepared and purified using high-performance liquid chromatography as described by Dyer et al. [100]. After validating the protein purity using sodium dodecyl sulfate (SDS) polyacrylamide gel electrophoresis (PAGE), fig. 11, the purified protein was dialyzed against deionized $\mathrm{H}_{2} \mathrm{O}$ and concentrated for octamer reconstitution.

\section{DNA Template Preparation and Purification}

DNA templates were designed using repeats of the W601 DNA sequence [4] because of its strong positional binding resulting in regularly spaced nucleosome arrays. Dr. Chongli Yuan, from Purdue University, provided DNA templates for the $3 \times 167$, and $4 \times 167$ arrays which were designed to be excised from a pUC19 vector using EcoRV restriction enzyme. Dr. Sergei Grigoryev, from Pennsylvania State University, provided the $2 \times 167$ DNA template, designed to be excised from a pUC19 vector using XbaI and KpnI restriction enzymes. All recombinant plasmids were transformed into E. coli DH5a [101] and grown in LB media with ampicillin or carbenicillin. After digestion, native PAGE gels were run to separate insert and vector DNA. After

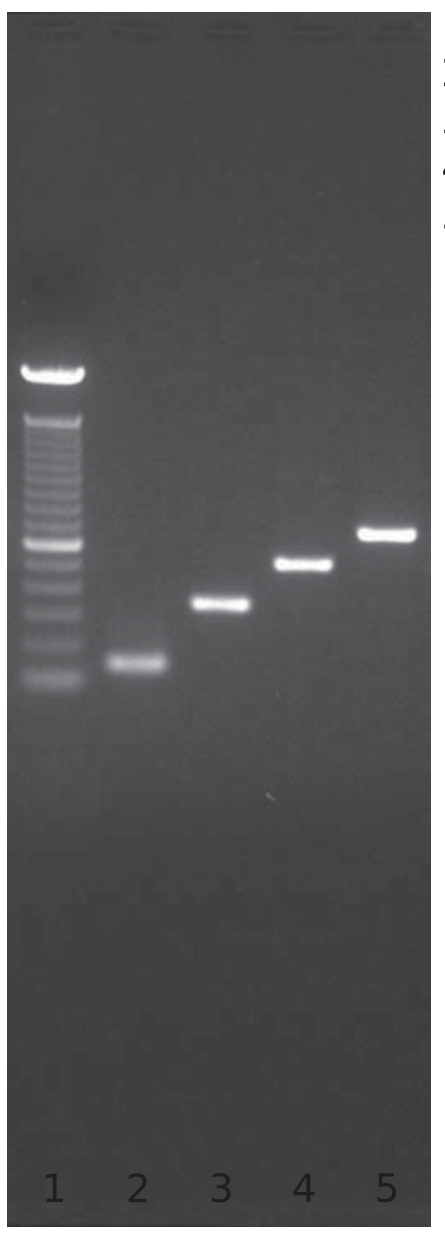

1. Ladder

2. MMTV

3. $2 \times 167$

4. $3 \times 167$

5. $4 \times 167$

Figure 12. Agarose Gel of the Purified DNA Templates: The single band for each sample lane indicates that the DNA template solutions are pure. 
separation, the gel was cut to isolate the pieces containing the DNA fragments. Gel pieces were placed in dialysis membrane then the DNA was eluted by applying $100 \mathrm{~V}$ in a standard electrophoresis apparatus. The eluted DNA was precipitated using ethanol then redissolved in $2 \mathrm{mM}$ Tris buffer. Additionally, 147 bp DNA fragments containing the MMTV DNA sequence (templates also provided by Dr. Yuan) were prepared in the same manner for use as a carrier DNA in the nucleosome reconstitution. Figure 12 shows an agarose gel verifying the purity of the final DNA templates. All $12 \times 167$ arrays, as well as the $4 \times 167$ arrays with gH5, were prepared by Bing-Rui Zhou and Yawen Bai from the Laboratory of Biochemistry and Molecular Biology at the National Cancer Institute (NIH), and consequently the quality control gels are not shown.

\section{Octamer Reconstitution and Purification}

Octamer reconstitution was performed using concentrated $\mathrm{H} 2 \mathrm{~A}, \mathrm{H} 2 \mathrm{~B}, \mathrm{H} 3$, and $\mathrm{H} 4$

proteins that were denatured using $6 \mathrm{M}$ guanidine hydrochloride then mixed at a molar ratio of 1:1:0.95:0.95 and dialyzed overnight against refolding buffer. Approximately $15 \mathrm{mg}$ of each histone was used for octamer reconstitution. After dialysis, octamers were purified using Superdex 200 gel filtration column with a flow rate of $1 \mathrm{~mL} / \mathrm{min}$. Before the purification, the column was equilibrated with 2 column volumes of refolding buffer. In a typical run, $\sim 5-7 \mathrm{~mL}$ of sample was injected into the column. The ultraviolet (UV) absorbance at $280 \mathrm{~nm}$ was used to monitor the separation of the eluted octamers.

The purity of the eluted octamer samples was confirmed using SDS-PAGE, fig. 13, the details of which are as follows. Before being loaded into an 18\% SDS-PAGE gel, octamer samples were mixed with SDS sample buffer and boiled at $100^{\circ} \mathrm{C}$ for $5 \mathrm{~min}$ to completely denature the proteins. The electrophoresis was run at $120 \mathrm{~V}$ for 120 150 min. Gels were stained in a solution containing $0.1 \%$ Coomassie Brilliant Blue 


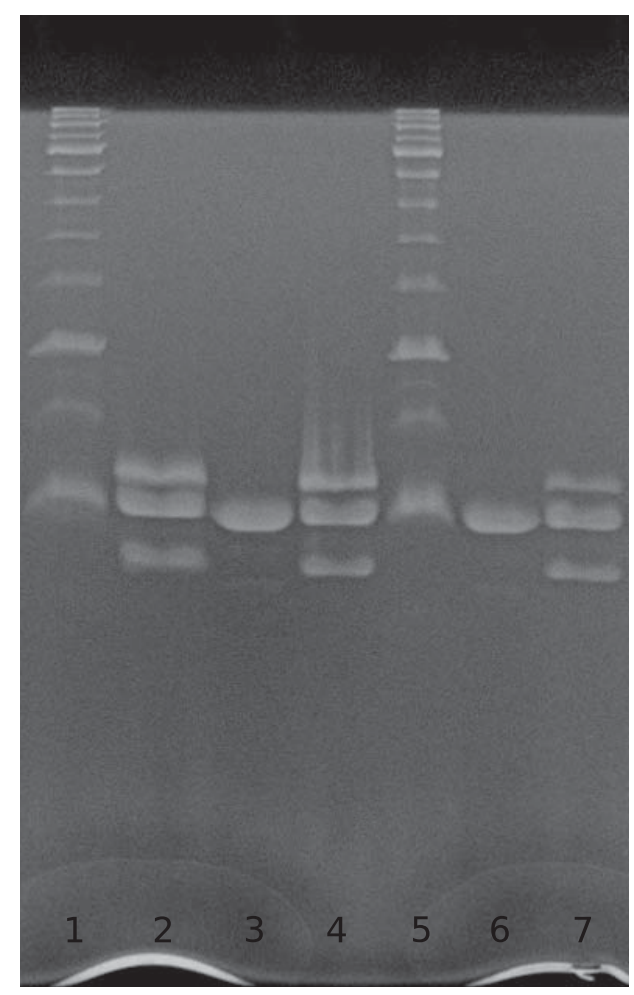

1. Ladder

2. Histone Octamer, Stock Mixture

3. H2A-H2B Dimer

4. Histone Octamer, Stock Mixture

5. Ladder

6. H2A-H2B Dimer

7. Histone Octamer, From NCP

Figure 13. SDS-PAGE Gel of the Combined Histone Proteins: The H2A-H2B dimers, in both lanes 3 and 6 , show minimal contamination of a small mass. Despite this, the histone octamers from the stock solution (lanes 2, 4) and from reconstituted nucleosomes (lane 7) show the expected ratios between the four core histones.

[102] and destained in a solution containing $10 \%$ acetic acid and 50\% methanol. After verifying their purity, octamers were stored on ice until nucleosome reconstitution.

\section{Nucleosome Array Reconstitution and Purification}

Reconstituted arrays were assembled using DNA templates and histone octamer. Reconstitutions were performed essentially as described by Dyer et al. [100]. The molar ratio for histone octamer, template DNA, and carrier DNA was 1.1:1:0.2 respectively. Samples were combined in a high salt buffer then successively dialyzed against $2,1.5$, 1, 0.5, and $0.01 \mathrm{M} \mathrm{K}^{+}$, each for 3 hours.

The reconstituted arrays were purified using differential centrifugation in a 5$20 \%$ sucrose gradient with $10 \mathrm{mM} \mathrm{K}^{+}$, centrifuged at $30000 \mathrm{rpm}$ for 16 hours at $4{ }^{\circ} \mathrm{C}$. After centrifugation, samples were divided into $500 \mu \mathrm{l}$ fractions and analyzed on a 


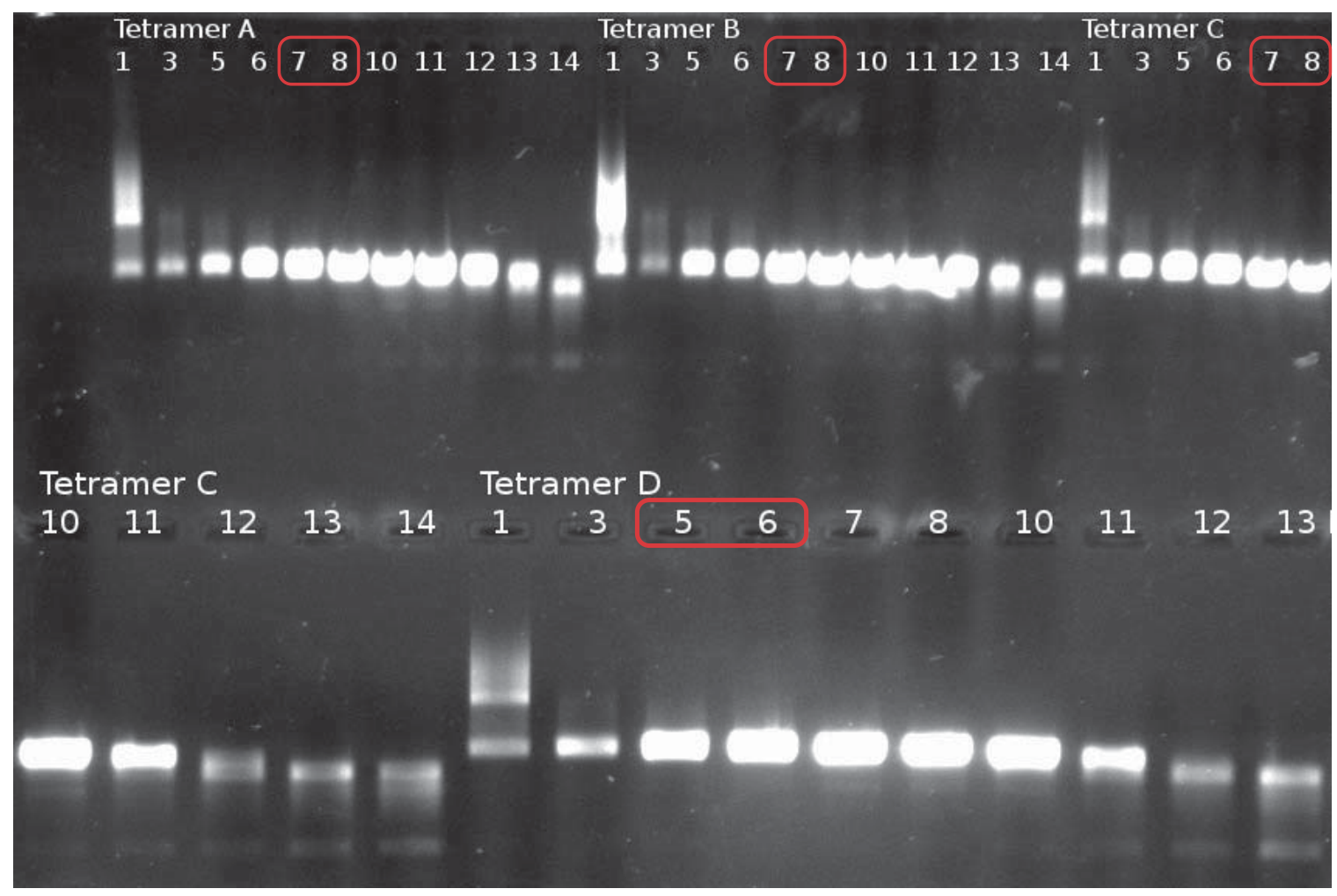

Figure 14. Example Agarose Gel from Differential Centrifugation: Increasing fraction number corresponds with decreasing sucrose concentration. Fractions highlighted in red were combined for the $4 \times 167$ samples measured at X9/NSLS. In each gradient, fraction 1 shows the presence of large aggregates, and fractions 10-14 contain the MMTV nucleosome; MMTV DNA was included to remove extra histone octamers. In the highest fraction lanes, 12-14, the strongest band represents underloaded arrays for which some W601 sites did not bind with a histone octamer.

$1 \%$ agarose gel at $90 \mathrm{~V}$ for $30 \mathrm{~min}$. Fractions containing pure arrays were pooled then dialyzed against $10 \mathrm{mM} \mathrm{K}^{+}$to remove sucrose.

Final SDS-PAGE gels were run to confirm the stability of histone octamer during nucleosome array reconstitution and purification. We also verified that all W601 nucleosome binding site were bound to histone octamers by digesting the linker DNA using the ScaI restriction enzyme, for $3 \times 167$ and $4 \times 167$, or EcoRV restriction enzyme, for $2 \times 167$, then running the result on a $1 \%$ agarose gel to check there was no free DNA. After all verifications, the final concentration of arrays was measured by spectrophotometry measurements at $260 \mathrm{~nm}$ then sample concentrations were adjusted appropriately for X-ray measurement. 


\section{SAXS Measurements}

\section{Experimental Configuration and Data Collection}

Solution SAXS experiments were carried out at both the G1 station of the CHESS in Ithaca, New York and the X9 beamline of the National Synchrotron Light Source (NSLS) in Brookhaven, New York. At G1/CHESS, the incident beam had an energy of $10.53 \mathrm{keV}$ and the sample-to-detector distance was $2.55 \mathrm{~m}$, measuring a $Q$-range of $0.0037 \leq Q \leq 0.2083 \AA^{-1}$. At X9/NSLS, the incident beam had an energy of $13.5 \mathrm{keV}$ and both small-angle and wide-angle X-ray scattering (WAXS) data were simultaneously measured at two distances [103] over the $Q$-ranges of $0.004 \leq Q \leq$ $0.135 \AA^{-1}$ and $0.105 \leq Q \leq 2.02 \AA^{-1}$ respectively. For both beamlines, an in-vacuum capillary flow cell was used to provide a windowless sample environment in order to reduce parasitic scattering. All measurements were performed at $20^{\circ} \mathrm{C}$.

To mitigate the effects of radiation damage, the sample solution was kept under oscillatory ( $\sim 30 \mu \mathrm{L}, \mathrm{G} 1 / \mathrm{CHESS})$ or continuous ( $60 \mu \mathrm{L}, \mathrm{X} 9 / \mathrm{NSLS})$ flow during the Xray exposure. To further prevent and verify the absence of radiation damage, exposure times were adjusted such that successive exposures of individual samples showed no systematic changes (2 seconds at G1/CHESS and 60 seconds at X9/NSLS), noting that, in the case of continuous flow at X9/NSLS, the measured sample solution was drawn back to its starting position for subsequent exposures. To account for possible artifacts caused by time-dependent beam conditions, e.g., linearly decreasing intensity over time, we measured the corresponding buffer both before and after each sample in order to average out systematic changes in the beam characteristics. Figure (15) shows the sample and buffer scattering profiles collected for $3 \times 167$ nucleosome arrays at a concentration of $0.5 \mathrm{mg} / \mathrm{mL}$ in $100 \mathrm{mM} \mathrm{\textrm {K } ^ { + }}$ at both G1/CHESS and X9/NSLS. The consistency in scattering over multiple exposures demonstrates the measurement reproducibility and the absence of radiation damage. 


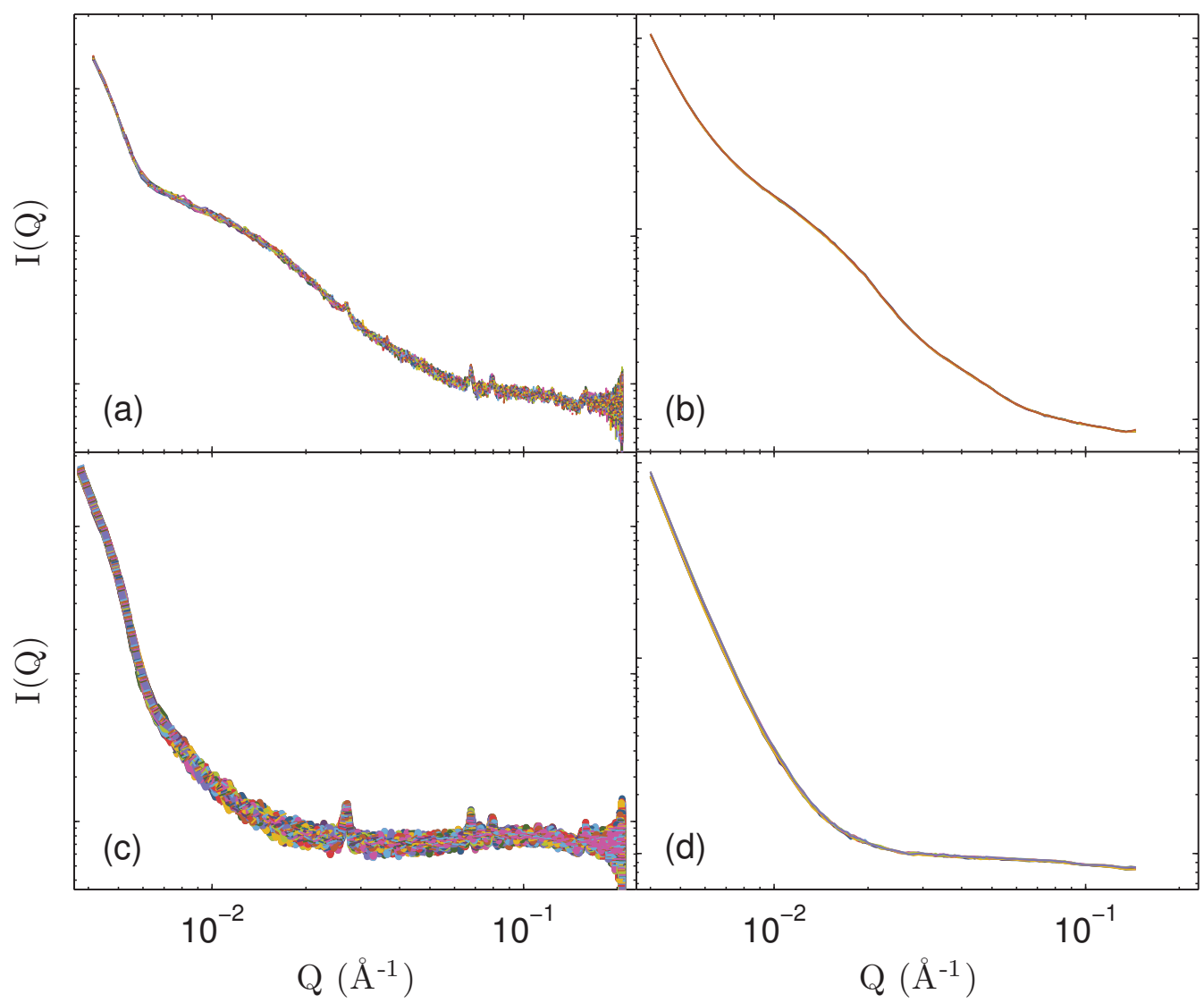

Figure 15. Raw Sample and Buffer Scattering Profiles: Successive SAXS data from sample, (a) and (b), and buffer, (c) and (d), solutions at G1/CHESS, (a) and (c), and X9/NSLS, (b) and (d). The consistency between repeated exposures demonstrates the instrument integrity and the lack of radiation damage under the conditions used for the measurements.

\section{Data Reduction and Buffer Subtraction}

As introduced in Chapter 1, scattered X-rays are recorded as a 2D image, and for samples that scatter isotropically the scattering intensity only depends on the scattering angle, or momentum transfer, $Q$. Consequently, the data can be reduced in dimensionality to a 1D plot through a process referred to as 'integrating', 'averaging', or 'binning' pixels within the annulus corresponding to a narrow $Q$-range.

In addition to simplifying data visualization and analysis, through this reduction process one can obtain the standard error of the scattering intensity for the pixels with the same $Q$ value. This standard error often provides a more reliable estimate for the 
actual uncertainty of the average intensity if this standard error exceeds the photon counting statistics-based error estimate calculated up to this step [104]. The Poissonbased photon counting statistics set the absolute minimum error in the recorded scattering intensity but do not account for other sources of error from the detector such as variance between pixel sensitivities, or electronic noise, nor sources of error from the X-ray source such as parasitic scattering, or beam fluctuations.

Algebraically, the average of the scattering intensities from individual pixels, $I_{j}$, in $Q_{\text {bin }}$ ranging from $\left[Q_{k}, Q_{k+1}\right]$, can be written as,

$$
I_{Q_{\mathrm{bin}}}(\bar{Q})=\left\langle I_{j}\left(Q \in\left[Q_{k}, Q_{k+1}\right]\right)\right\rangle
$$

where $I_{Q_{\text {bin }}}$ is the mean scattering intensity for the corresponding average $Q$-value,

$$
\bar{Q}=\left\langle Q \in\left[Q_{k}, Q_{k+1}\right]\right\rangle
$$

Following Pauw et al. [104], the best method of estimating the error for $I_{Q_{\text {bin }}}$ is to use,

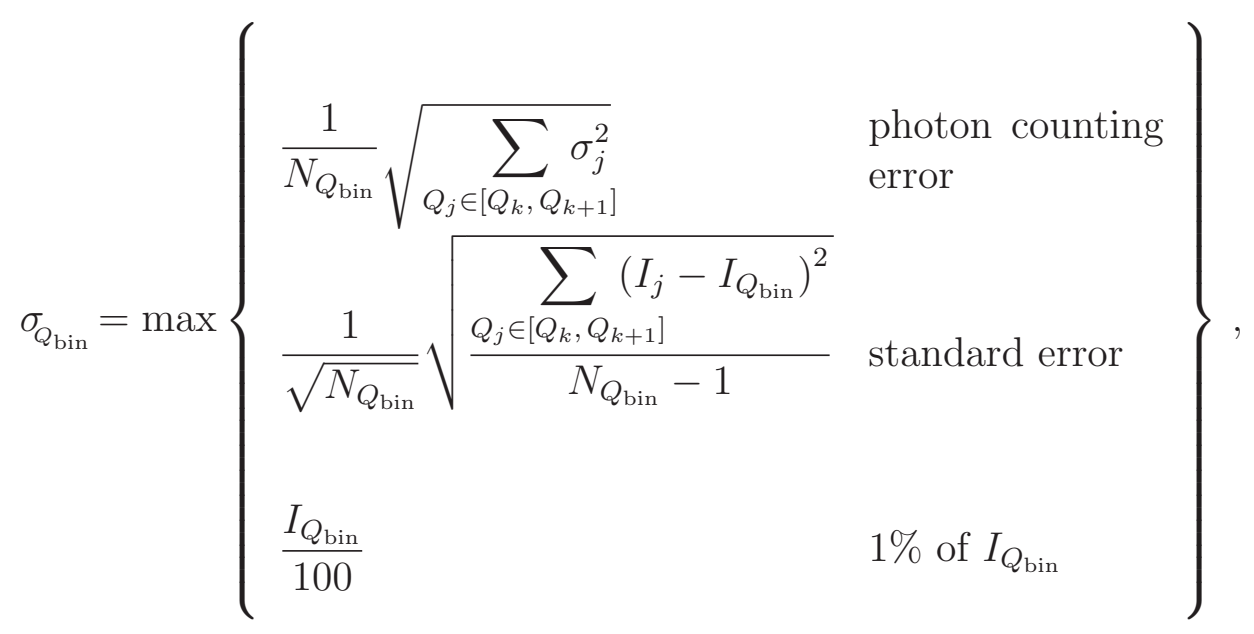

where $\sigma_{Q_{\mathrm{bin}}}$ is the error for the scattering intensity at the $Q=Q_{\text {bin }}$ grid point and each summation is over $N_{Q_{\text {bin }}}$ pixels, $j$, within the range $Q_{j} \in\left[Q_{k}, Q_{k+1}\right]$. To avoid 
underestimating the error, the maximum value is chosen from the propagated error originating from the Poisson photon counting statistics, the standard error of the mean, and $1 \%$ of the mean scattering intensity. The $1 \%$ minimum is included as, even when applying the most thorough corrections, it is difficult to achieve a higher level of accuracy than this [105]. Unfortunately, for both G1/CHESS and X9/NSLS, the reduction code only output the standard error of the mean. Rather than modifying the reduction code, because of time limitations, we simply compared the standard error to $1 \%$ of the intensity and used the maximum of these.

For improved statistics and reduced noise, we collected several exposures from each sample. After reducing the 2D images to $1 \mathrm{D}$ scattering intensity profiles using eqs. (21)-(23), we averaged this 1D data, i.e.,

$$
\bar{I}(Q)=\frac{1}{N_{e}} \sum_{n=1}^{N_{e}} I_{n}(Q),
$$

where $N_{e}$ is the number of repeated exposures. We combined the error from each 1D data set in a manner similar to that used when combining pixel error. In this case, we used the maximum of either the propagated error from the individual exposures or the standard error of the variance between exposures. Analytically this takes the form,

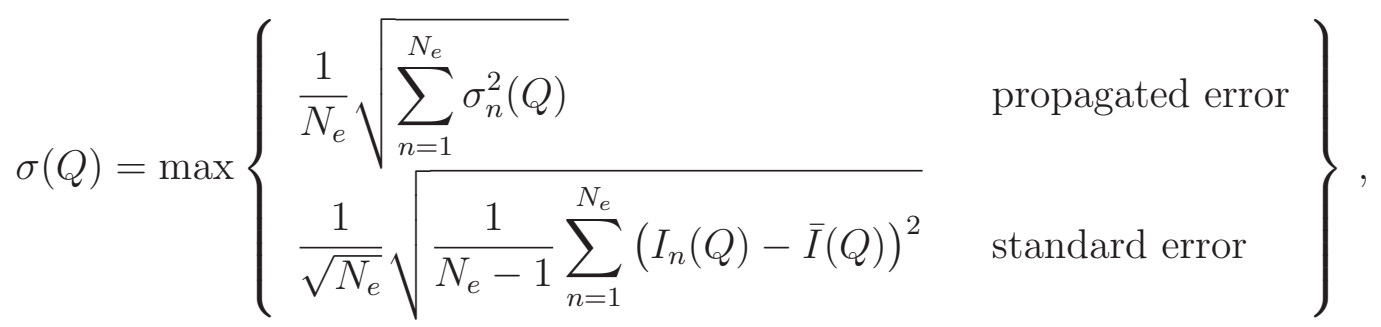

where $\sigma(Q)$ is the error in the scattering intensity. ${ }^{5}$

\footnotetext{
5 The propagated error in eq. (25) assumes that the number of pixels for each $Q$ point was the same or nearly so. This is always the case for standard SAXS configurations as it would be extremely unusual to change the experimental configuration during an exposure. If this were to happen unintentionally, it would be simpler to repeat the measurement than to complicate the reduction process by trying to recover the data.
} 


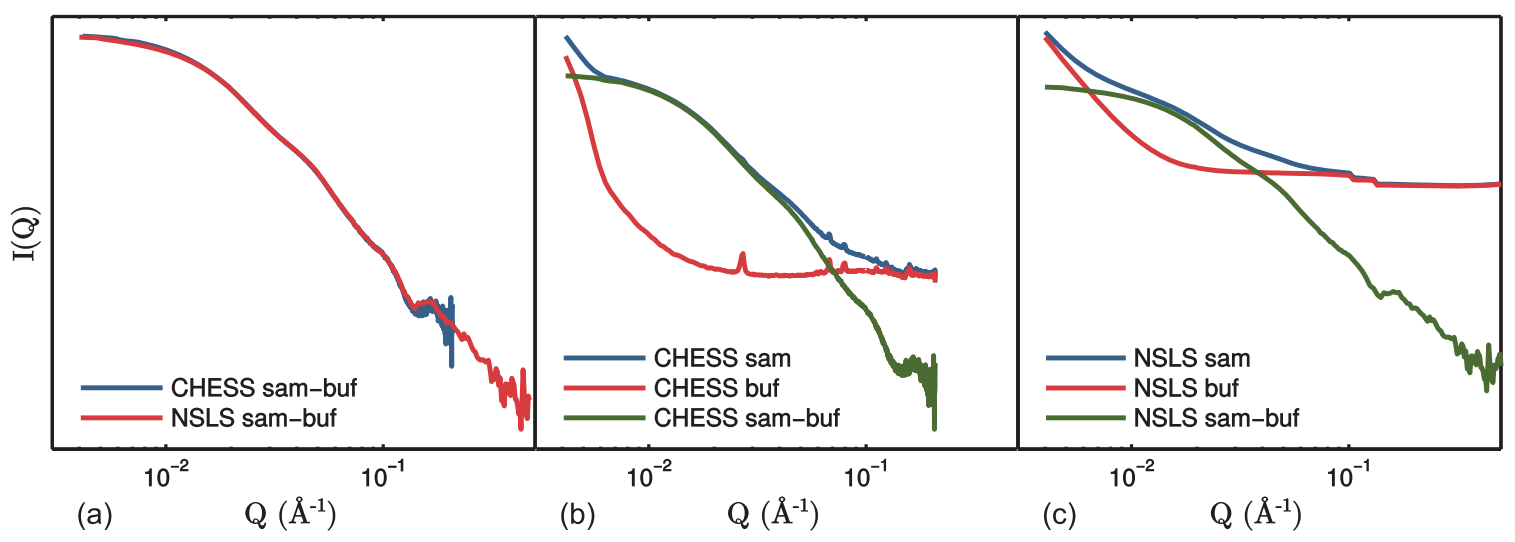

Figure 16. SAXS Buffer Subtraction: Buffer subtraction example from $0.5 \mathrm{mg} / \mathrm{mL}$ solutions of $3 \times 167$ arrays in $100 \mathrm{mM} \mathrm{K}^{+}$. (a) comparing the buffer subtracted scattering intensities from G1/CHESS, (b), and X9/NSLS, (c), demonstrates excellent agreement between the two measurements. The scattering from both G1/CHESS and X9/NSLS contain systematic features but, because they are independent of the sample they are not present after subtracting the buffer data from the sample data.

For the G1/CHESS data, we performed this reduction and averaging process using MATLAB ${ }^{\circledR}$ scripts. As a preliminary correction, prior to reducing the data we normalized each individual exposure using the transmitted beam intensity recorded by a PIN diode mounted on the beamstop. After this correction, we reduced and combined the exposures for each sample or buffer, then subtracted the average buffer scattering from the average sample scattering.

We processed the X9/NSLS data in a similar manner using python scripts developed for the beamline [103]. We scaled and merged the WAXS and SAXS data using the overlapping region, $0.105 \AA^{-1}<Q<0.135 \AA^{-1}$, then reduced and combined exposures for each sample or buffer. We finally normalized the buffer scattering with respect to the sample scattering using the water scattering peak in the WAXS data, centered at $Q=1.735 \AA^{-1}$. In fig. 16 we show the buffer subtraction for an example data sets taken from $0.5 \mathrm{mg} / \mathrm{mL} 3 \times 167$ arrays in $100 \mathrm{mM} \mathrm{K}^{+}$, measured at both G1/CHESS and X9/NSLS. 


\section{Determining the Form Factor}

Recall that the measured scattering intensity $I(Q)$ is the product of the single-particle form factor, $P(Q)$, and the interparticle structure factor, $S(Q)$, described in Chapter 1 , and that by measuring molecules at sufficiently low sample concentration the inter-molecular interactions become negligible, leaving $I(Q)=P(Q)$ (eq. (5)). The typical practice for verifying samples are sufficiently dilute is to measure a series of decreasing sample concentrations then make certain that interparticle interactions are not present in the data. As the concentration decreases, the low- $Q$ scattering, encoding large length scale structure features, will change as particle interactions become less frequent. ${ }^{6}$ The high- $Q$ scattering will not change with concentration as smaller length scales structures are independent of interparticle repulsion or attraction. By comparing measurements from different concentrations, one can identify at what point the low- $Q$ scattering no longer changes, indicating that the average distance between particles is such that there are essentially no remaining interparticle interactions.

On the other hand, excessively low sample concentrations result in a deteriorated SNR as the buffer and parasitic scattering remains constant. A recommended "ruleof-thumb" for determining the lower limit for obtaining high-quality signal is that the product of the concentration and molecular weight in units of $\mathrm{mg} / \mathrm{mL}$ and $\mathrm{kDa}$ should be above 100 [106]. This means a single nucleosome with a molecular weight of $\sim 200 \mathrm{kDa}$ should provide sufficient scattering when measured at a concentration greater than $0.5 \mathrm{mg} / \mathrm{mL}$. Similarly, the lower limit for measuring an array of 2, 3, 4, or 12 nucleosomes should respectively be $0.25,0.17,0.13$, and $0.04 \mathrm{mg} / \mathrm{mL}$.

In an effort to eliminate interparticle interactions while also having a sufficient SNR, we measured each construct at three sample concentrations below $1 \mathrm{mg} / \mathrm{mL}$, typically $0.5,0.25$, and $0.125 \mathrm{mg} / \mathrm{mL}$. It is important to note that the concentration

\footnotetext{
${ }^{6}$ Recall the Bragg equation, eq. (17), which states that $Q$ is inversely proportional to size.
} 
of the measured sample varied slightly from the prepared concentration because of dead volume in the flow cell. Despite drying the capillary using compressed air between sample changes, we observed deviations in the total amount of scattering for samples with the same prepared concentration measured for the same amount of time, indicating concentration deviations, discussed further in appendix B. Our measurements indicate the "rule-of-thumb" for the lower limit applies reasonably well to nucleosomes, as our measurements at or below that limit showed insufficient SNR at the extreme $Q$-values. Particularly, at $Q \lesssim 0.006 \AA^{-1}$, the overall scattering intensity for the lower concentrations decreased to the point that the resulting signal was often dominated by a small amount of beam spill-over around the beam stop or inconsistent slit scattering, both producing erroneous data. As a synchrotron beam position shifts slightly over the hours of measuring multiple samples, this contaminated signal was not consistently in the same detector pixels nor was it consistently observed all low concentration measurements. Rather than excessively masking out the pixels at these lowest $Q$-values for all data, we removed these points from the final 1-D data based on the Guinier fit of the $R_{g}$. The decrease in SNR observed at $Q \gtrsim 0.1 \AA^{-1}$ was not a significant concern as the scattering in this $Q$-range did not depend on concentrations so the standard practice is to use the data with the best SNR for these $Q$-values.

Using the three different concentration measurements, we performed a zero concentration extrapolation to eliminate any scattering contribution from interparticle interactions at low- $Q$. This extrapolation of $I(Q)$ treats the interaction effects as a linear function of the concentration. Through this process, we obtained the effective scattering of the infinitely dilute case, or zero concentration.

Figures 17-20 illustrate this extrapolation for the $2 \times 167,3 \times 167$, and $4 \times 167$ arrays. Similar figures for the $12 \times 167$ arrays are shown in appendix B. These plots are truncated at the minimum $Q$ used for the Guinier fit of the zero concentration extrapolation; lower $Q$-values are not included because of the insufficient SNR. We 
note that we exclude the $0.9 \mathrm{mg} / \mathrm{mL}$ measurement from the $4 \times 167$ array with gH5 in $1 \mathrm{mM} \mathrm{Mg}^{2+}$ as this data varied drastically compared to the 0.35 and $0.87 \mathrm{mg} / \mathrm{mL}$ data at $Q<0.011 \AA^{-1}$ (shown in fig. 24).

In each figure, panel (a) shows $I(Q)$ from the 3 measured concentrations as well as $P(Q)$ from the extrapolation result. We include an inset highlighting the low- $Q$ region as this encodes the dimensions affected by interparticle interactions. The dashed vertical gray lines indicate the $Q$-values used for scaling the lower concentration data, discussed in more detail in the subsequent section. Panel (b) compares the prepared concentration and the concentration determined from the scale factor applied to each $I(Q)$. Panel (c) shows the effective second virial coefficient, the coefficient of the first-order term in the Taylor series expansion of the interaction potential.
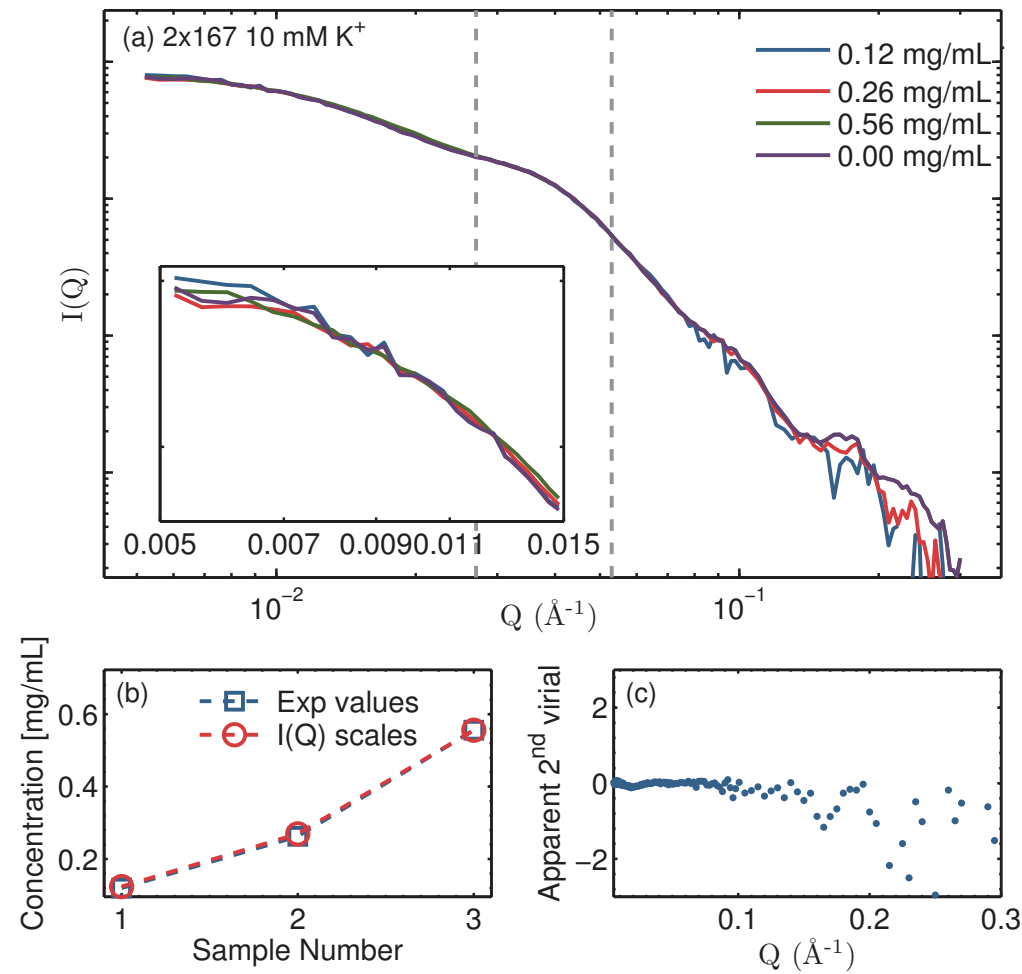

Figure 17. Zero Concentration Extrapolation for $\mathbf{2} \times \mathbf{1 6 7}$ Arrays: (a) the reduced scattering intensity with an inset zoomed in on the lowest $Q$-range, both plotted using logarithmic scales. The vertical lines indicate the $Q$-range used for scaling the data before performing the extrapolation. (b) comparison between the prepared concentration and the relative concentration determined by the scale factor used in (a). (c) the apparent second virial coefficient. 

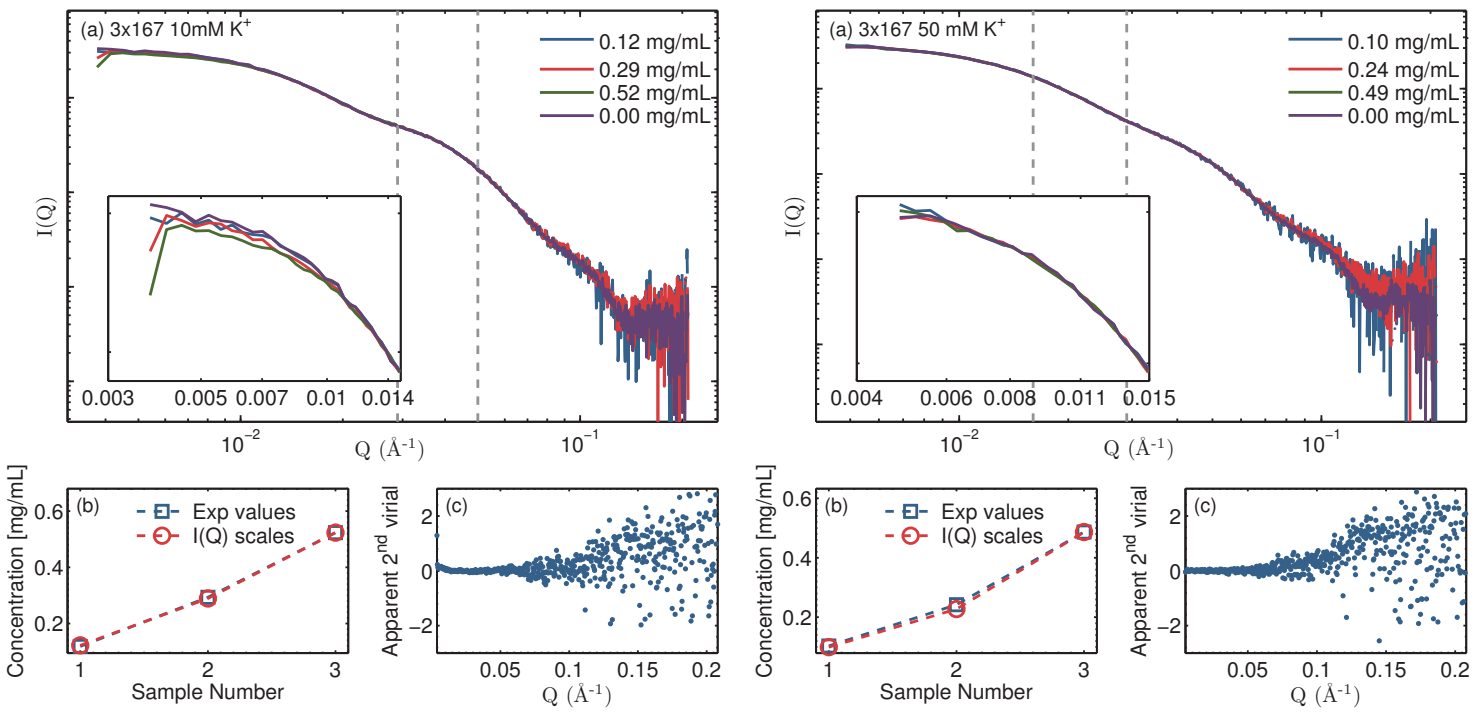

(i) $10 \mathrm{mM} \mathrm{K}^{+}$

(ii) $50 \mathrm{mM} \mathrm{K}^{+}$

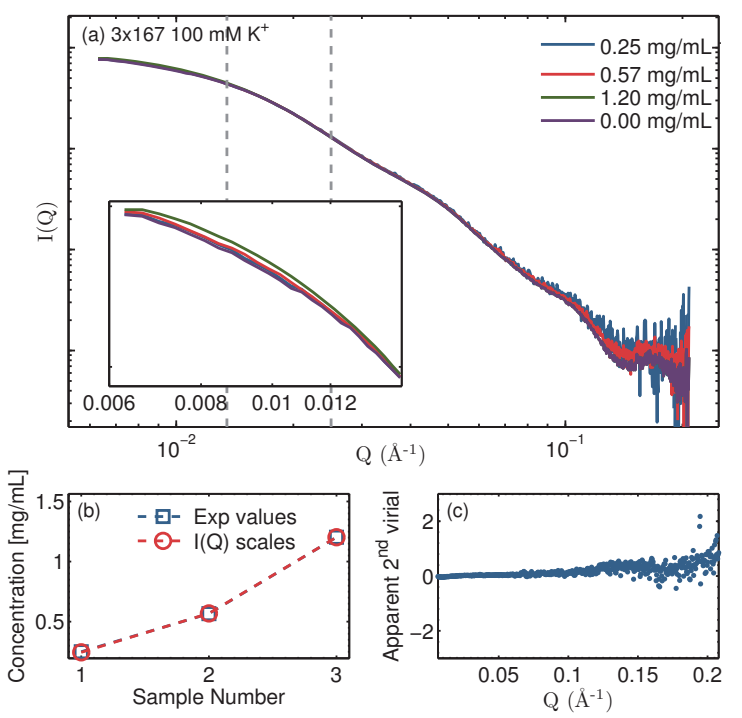

(iii) $100 \mathrm{mM} \mathrm{K}^{+}$
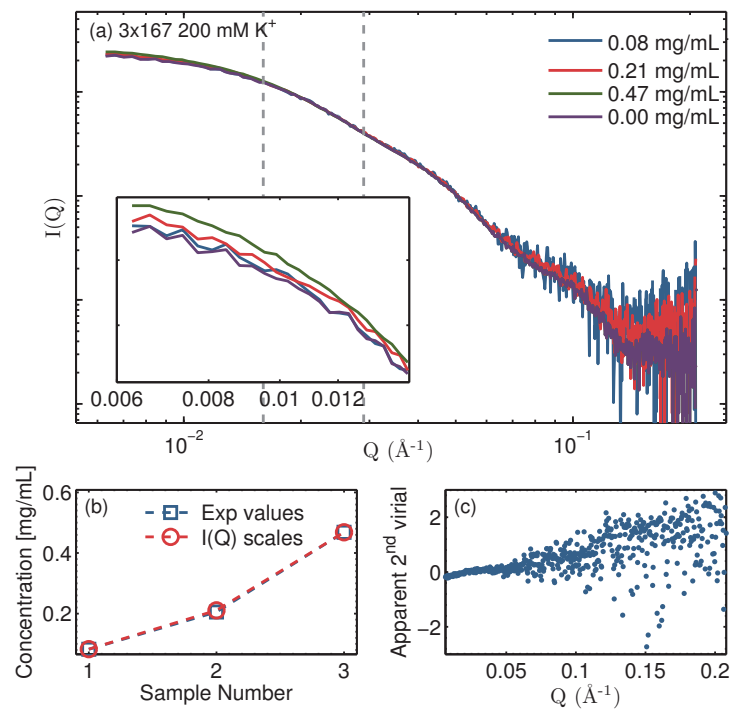

(iv) $200 \mathrm{mM} \mathrm{K}^{+}$

Figure 18. Zero Concentration Extrapolation for $3 \times 167$ Arrays: (a) $-(\mathrm{c})$ as in fig. 17, namely: (a) the reduced scattering intensity with an inset zoomed in on the lowest $Q$-range, both plotted using logarithmic scales. The vertical lines indicate the $Q$-range used for scaling the data before performing the extrapolation. (b) comparison between the prepared concentration and the relative concentration determined by the scale factor used in (a). (c) the apparent second virial coefficient. 


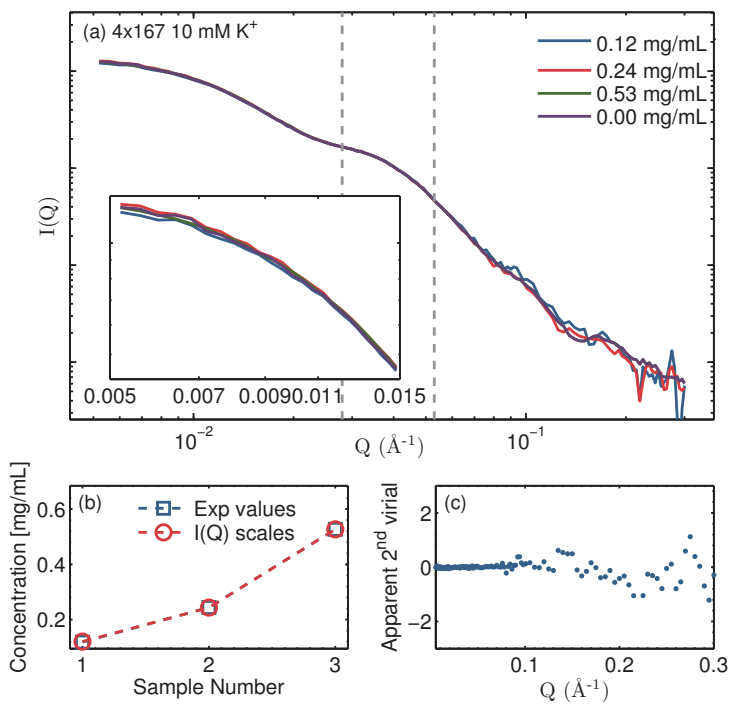

(i) $10 \mathrm{mM} \mathrm{K}^{+}$

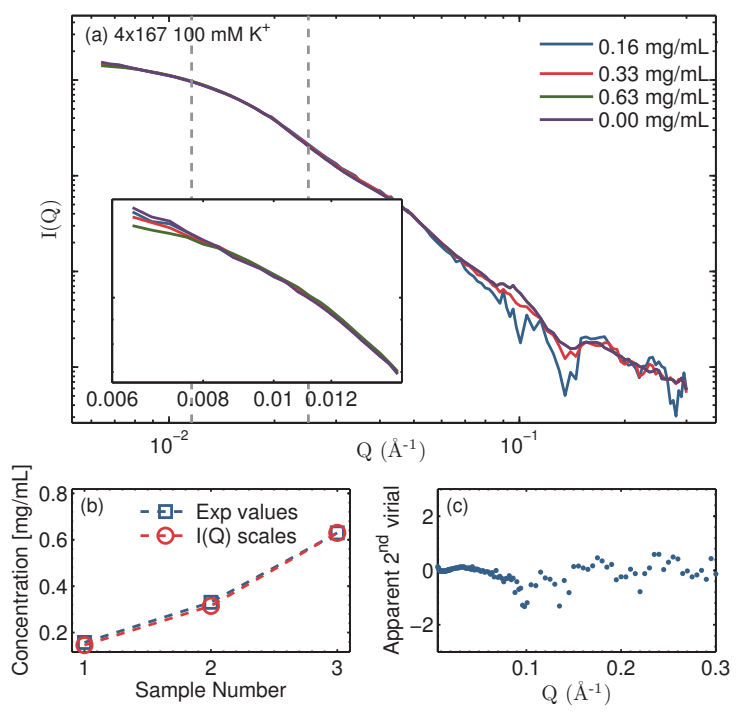

(iii) $100 \mathrm{mM} \mathrm{K}^{+}$
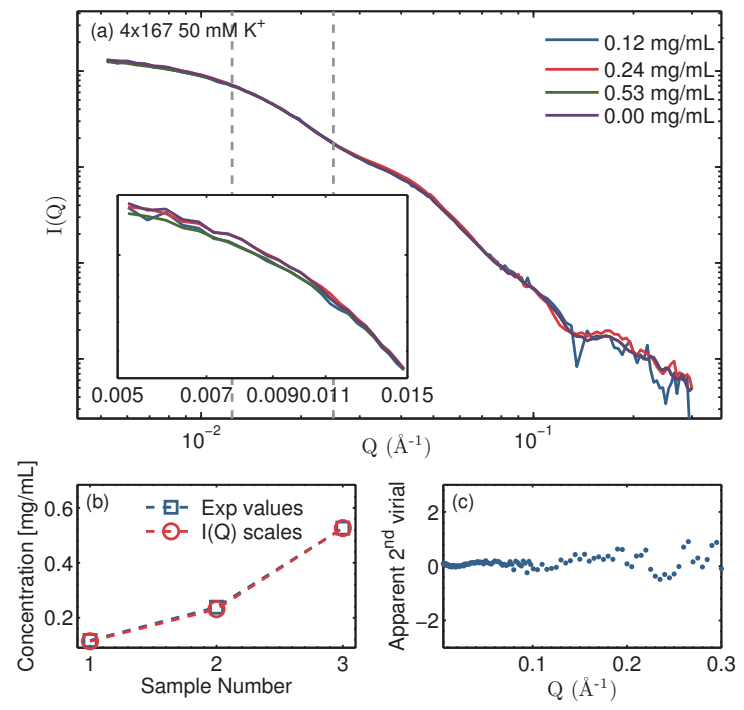

(ii) $50 \mathrm{mM} \mathrm{K}^{+}$

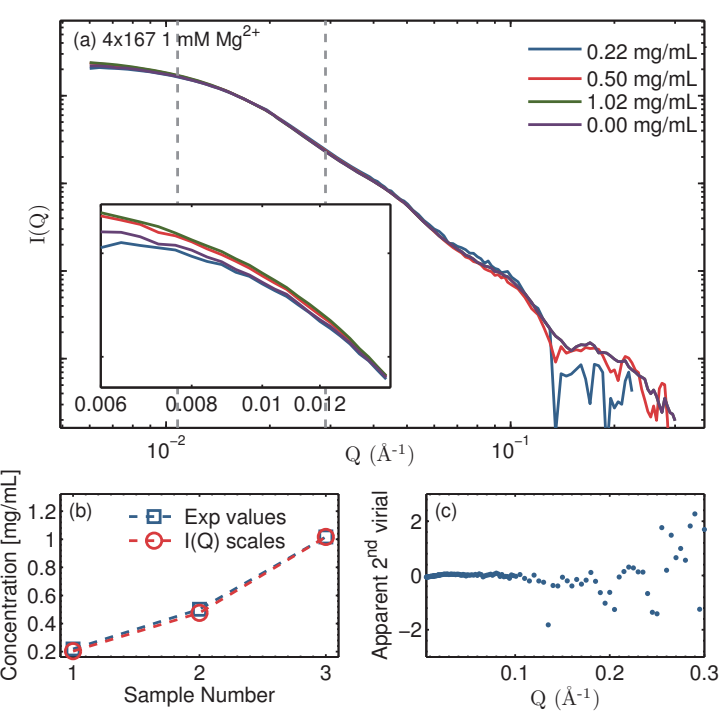

(iv) $1 \mathrm{mM} \mathrm{Mg}^{2+}$

Figure 19. Zero Concentration Extrapolation for $4 \times 167$ Arrays: (a)-(c) as in figs. 17 and 18, namely: (a) the reduced scattering intensity with an inset zoomed in on the lowest $Q$-range, both plotted using logarithmic scales. The vertical lines indicate the $Q$-range used for scaling the data before performing the extrapolation. (b) comparison between the prepared concentration and the relative concentration determined by the scale factor used in (a). (c) the apparent second virial coefficient. 


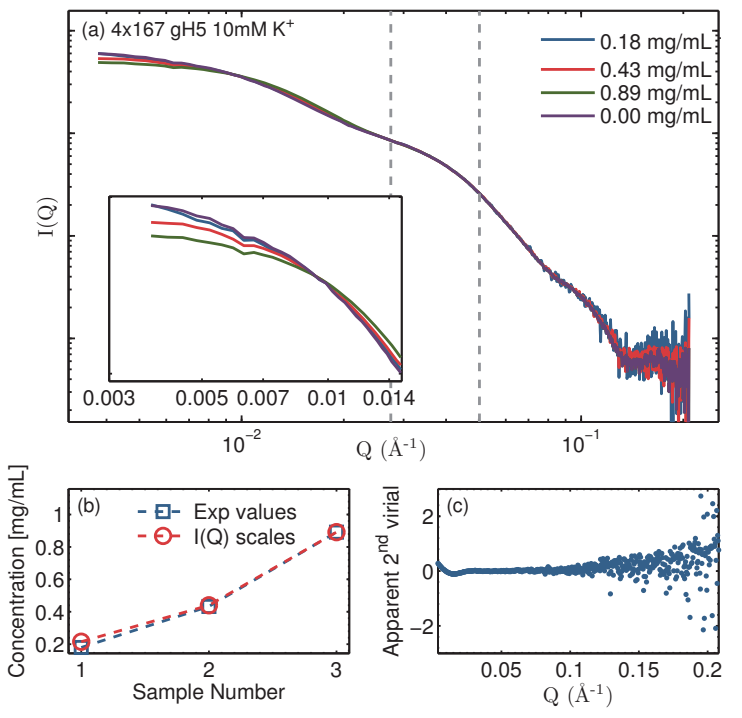

(i) $10 \mathrm{mM} \mathrm{K}^{+}$
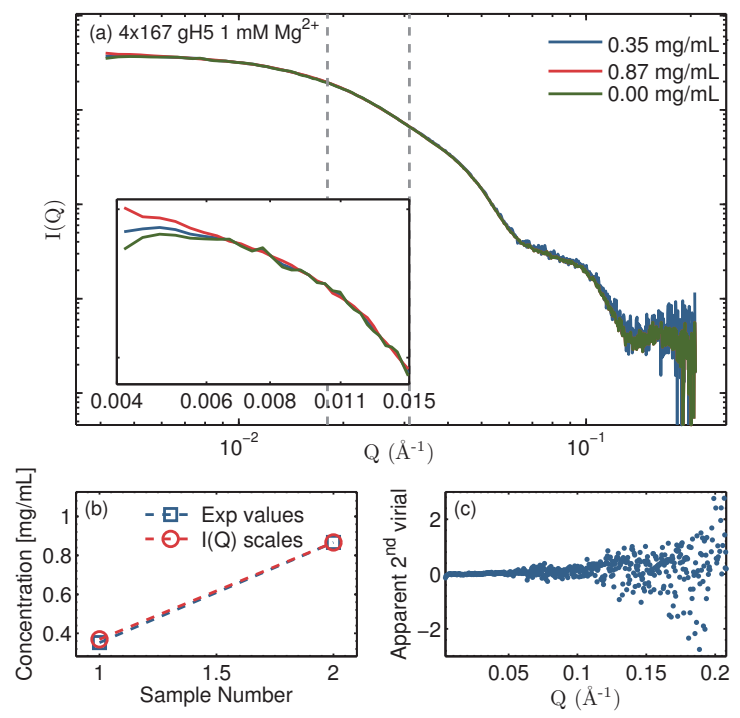

(ii) $1 \mathrm{mM} \mathrm{Mg}{ }^{2+}$

Figure 20. Zero Concentration Extrapolation for $\mathbf{4} \times \mathbf{1 6 7}$ gH5 Arrays: (a)-(c) as in figs. 17-19, namely: (a) the reduced scattering intensity with an inset zoomed in on the lowest $Q$-range, both plotted using logarithmic scales. The vertical lines indicate the $Q$-range used for scaling the data before performing the extrapolation. (b) comparison between the prepared concentration and the relative concentration determined by the scale factor used in (a). (c) the apparent second virial coefficient.

\section{Reduced SAXS Profiles}

To compare the $I(Q)$ from each sample concentration, figs. 21-24 compare the $I(Q)$ from each sample concentration and the zero concentration extrapolations for each construct-salt combination measured. Similar figures for the $12 \times 167$ arrays are shown in appendix B. Four plots are shown for each array construct in each salt concentration: (a) shows the reduced $I(Q)$ using a linear scale for both axes, (b) shows the same $I(Q)$ with error bars using a logarithmic scale for both axes, (c) shows a Guinier plot with $R_{g}$ fits from the lowest usable $Q$-point up to $Q_{\max }=1.3 / R_{g}$, (d) plots the data as $Q^{2} I(Q)$ versus $Q$, known as a Kratky plot [72]. The dashed vertical lines in (a), (b), and (d) indicate the $Q$-range used for scaling the lower concentration data for improved comparison. 
This same $Q$-range was also used for the zero concentration extrapolations as well as for merging the zero concentration $P(Q)$ with the highest concentration $I(Q)$. Each $Q$-range was individually selected using the principle peak in the Kratky plot as this is the first clear structure feature in the data. For merging the data, we used a linear weight factor, $w_{i}$, which can be written analytically as,

$$
w_{i}=\frac{i}{\left(N_{Q}-1\right)},
$$
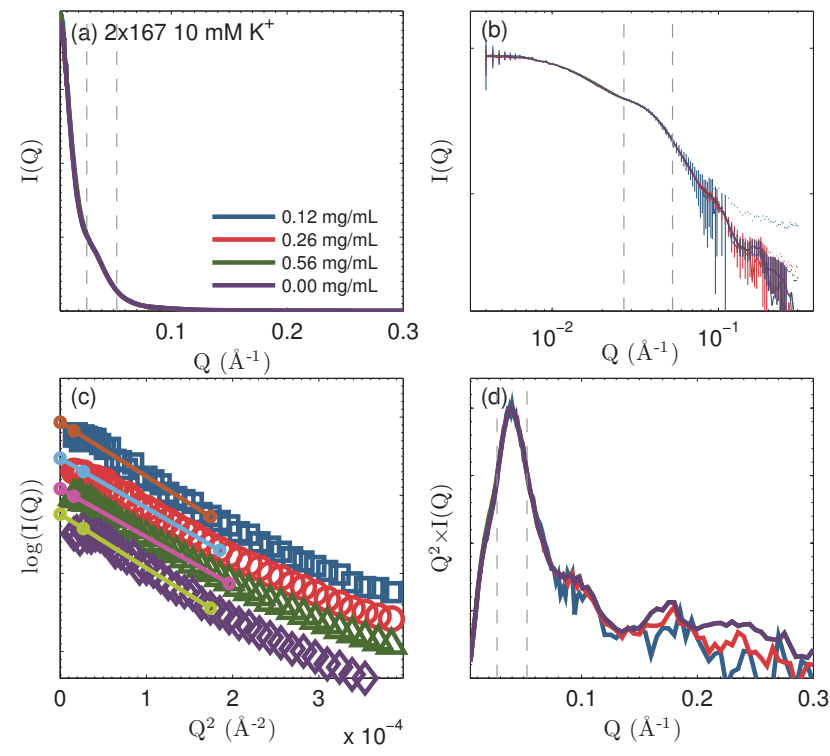

Figure 21. Reduced SAXS Profiles for $2 \times 167$ Arrays: (a)-(b) $I(Q)$ plots respectively on a linear and logarithmic scale. (c) Guinier plot with $R_{g}$ fits from the lowest usable $Q$-point up to $Q_{\max }=1.3 / R_{g}$. (d) Kratky plot, emphasizing the low- $Q$ features. The dashed vertical lines indicate the $Q$-range used for scaling the data.

where $i=0, \ldots, N_{Q}-1$, and $N_{Q}$ is the number of $Q$-points in the merge range. The expression for applying this weight factor is written as,

$$
I_{m}\left(Q_{i}\right)=\left(1-w_{i}\right) I_{z}\left(Q_{i}\right)+w_{i} I_{h}
$$

where $I_{m}, I_{z}$, and $I_{h}$ are respectively the merged, zero concentration, and high concentration scattering intensities. The error is combined in a similar manner using,

$$
\sigma_{m}\left(Q_{i}\right)=\sqrt{\left(1-w_{i}\right)^{2}\left(\sigma_{z}\left(Q_{i}\right)\right)^{2}+w^{2}\left(\sigma_{h}\left(Q_{i}\right)\right)^{2}}
$$

where $\sigma_{m}\left(Q_{i}\right), \sigma_{z}\left(Q_{i}\right)$, and $\sigma_{h}\left(Q_{i}\right)$ are respectively the merged, zero concentration, and high concentration error estimates at the $i^{\text {th }} Q$-point. Under the experimental limitations, this reduced $I_{z}(Q)$ is the best approximation to $P(Q)$. 

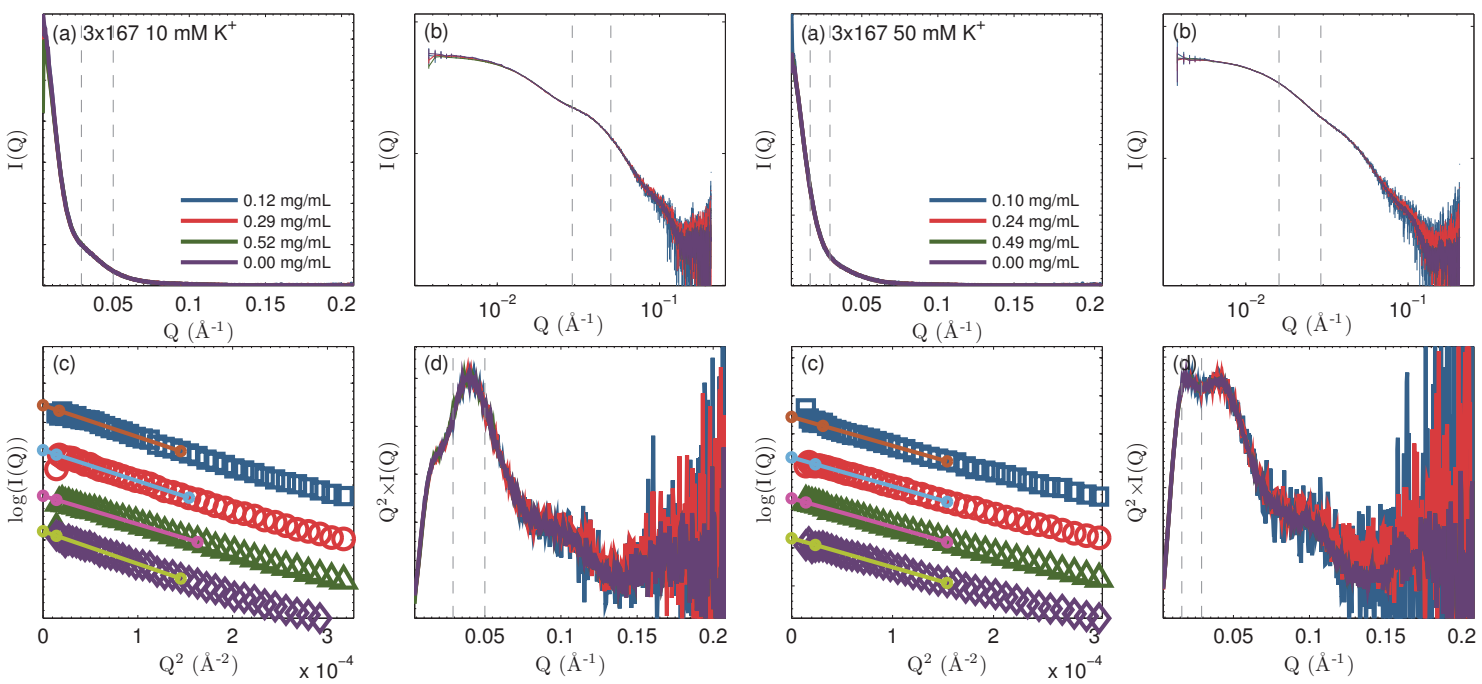

(i) $10 \mathrm{mM} \mathrm{K}^{+}$
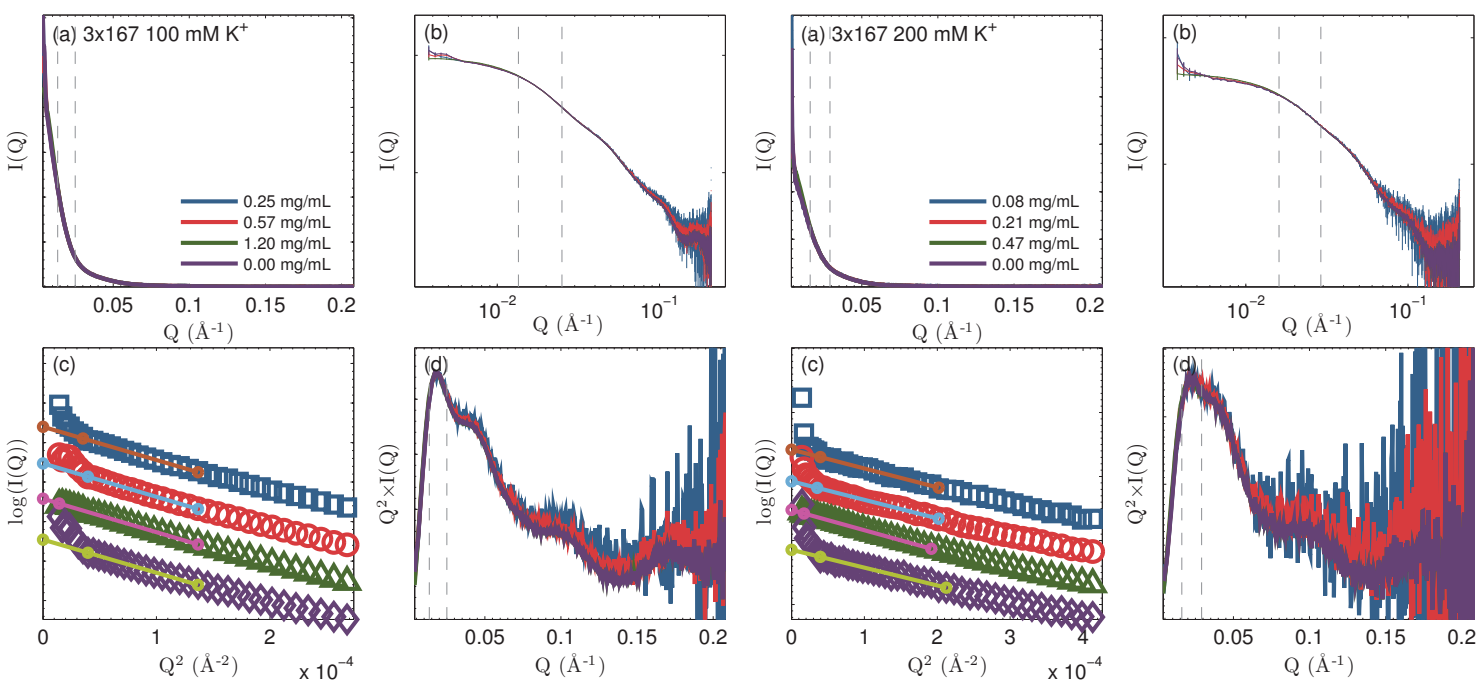

(iii) $100 \mathrm{mM} \mathrm{K}^{+}$
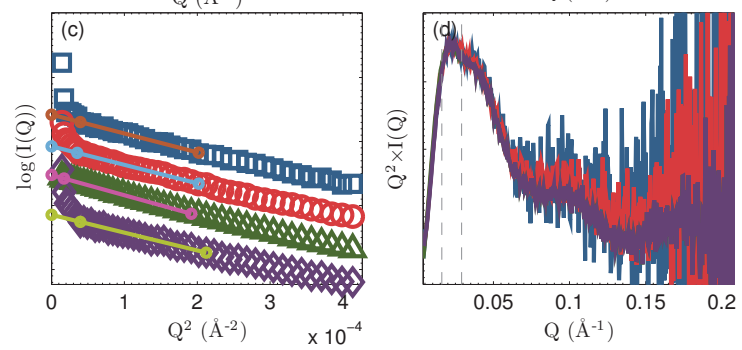

(iv) $200 \mathrm{mM} \mathrm{K}^{+}$

Figure 22. Reduced SAXS Profiles for $\mathbf{3} \times \mathbf{1 6 7}$ Arrays: (a)-(d) as in fig. 21, namely: (a)-(b) $I(Q)$ plots respectively on a linear and logarithmic scale. (c) Guinier plot with $R_{g}$ fits from the lowest usable $Q$-point up to $Q_{\max }=1.3 / R_{g}$. (d) Kratky plot, emphasizing the low- $Q$ features. The dashed vertical lines indicate the $Q$-range used for scaling the data. 

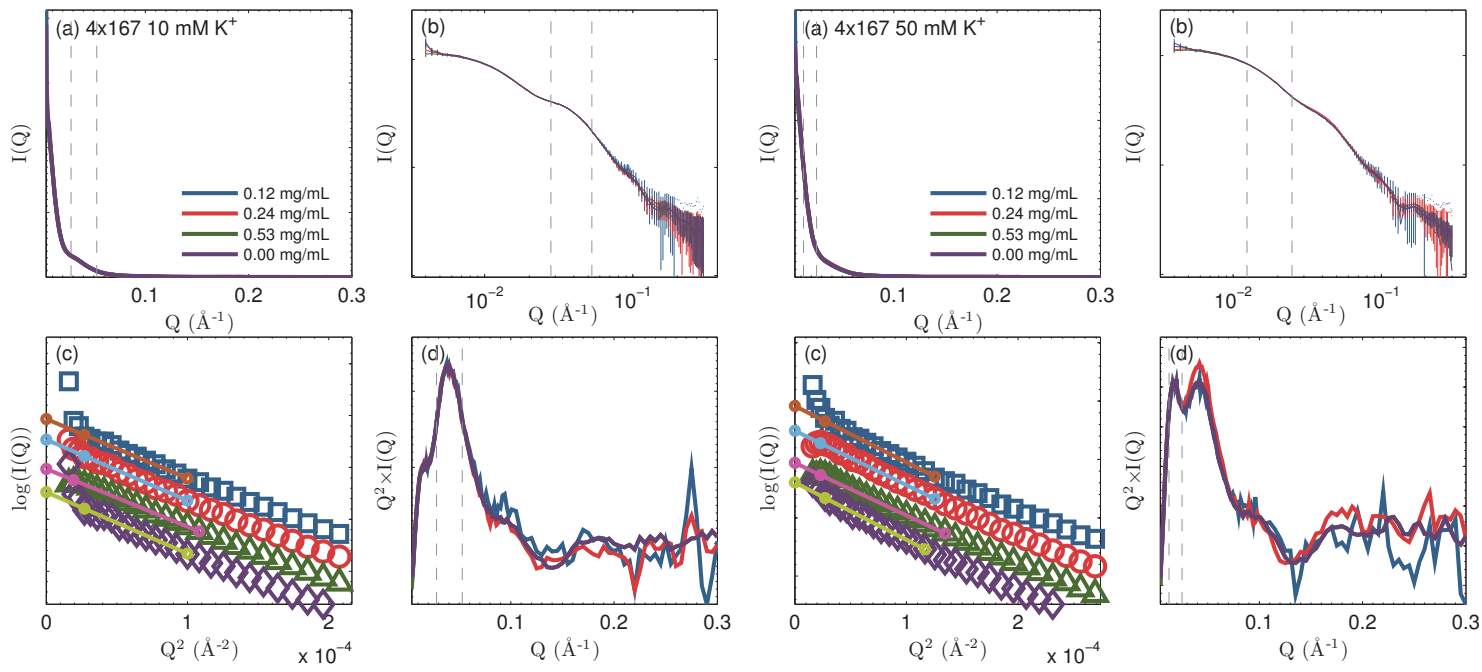

(i) $10 \mathrm{mM} \mathrm{K}^{+}$

(ii) $50 \mathrm{mM} \mathrm{K}^{+}$
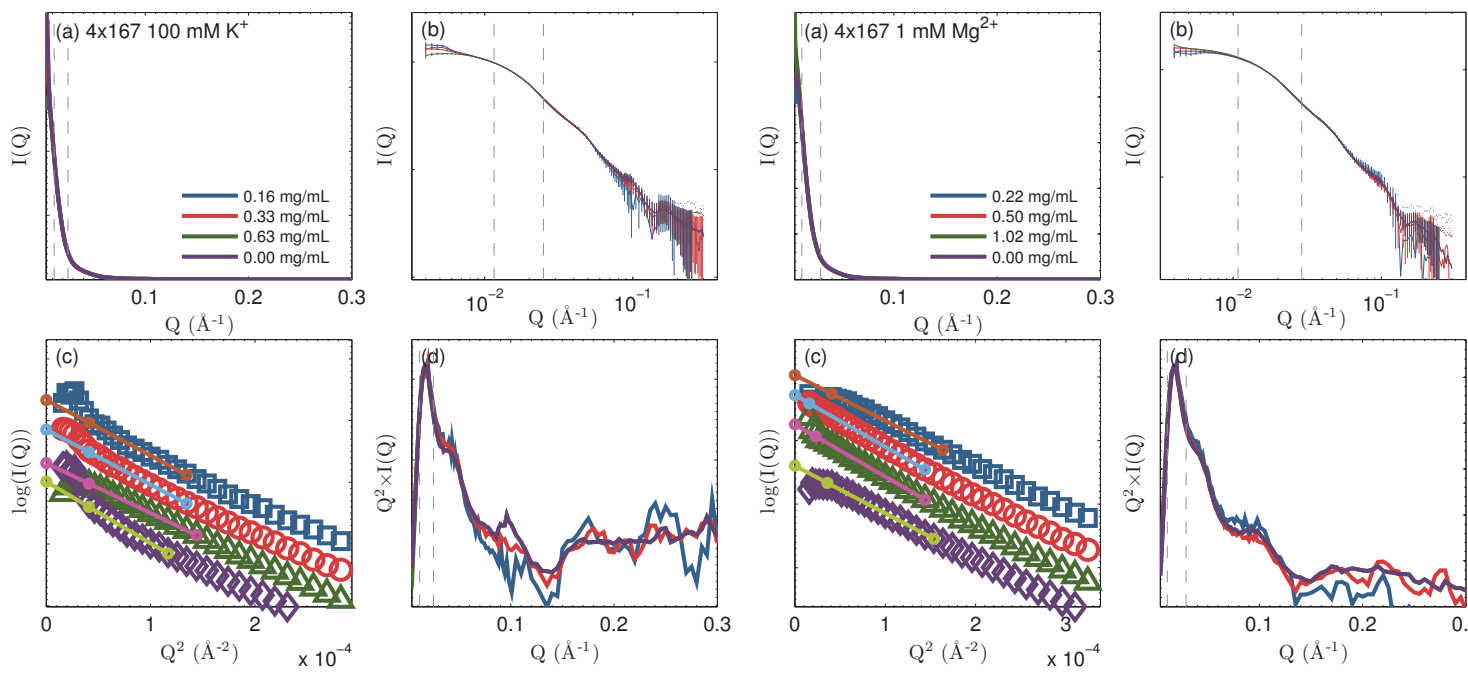

(iii) $100 \mathrm{mM} \mathrm{K}^{+}$
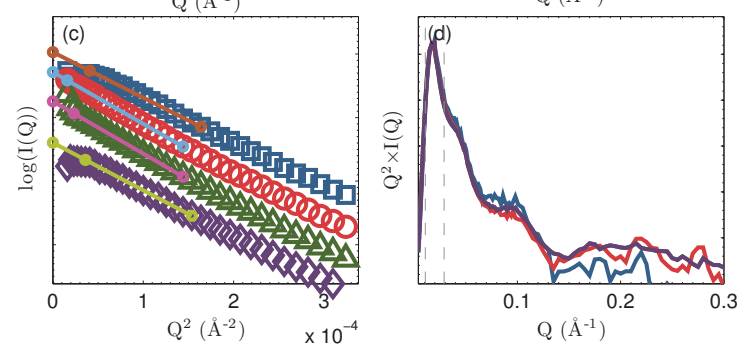

(iv) $1 \mathrm{mM} \mathrm{Mg}{ }^{2+}$

Figure 23. Reduced SAXS Profiles for $\mathbf{4} \times \mathbf{1 6 7}$ Arrays: (a)-(d) as in figs. 21 and 22, namely: (a)-(b) $I(Q)$ plots respectively on a linear and logarithmic scale. (c) Guinier plot with $R_{g}$ fits from the lowest usable $Q$-point up to $Q_{\max }=1.3 / R_{g}$. (d) Kratky plot, emphasizing the low- $Q$ features. The dashed vertical lines indicate the $Q$-range used for scaling the data. 

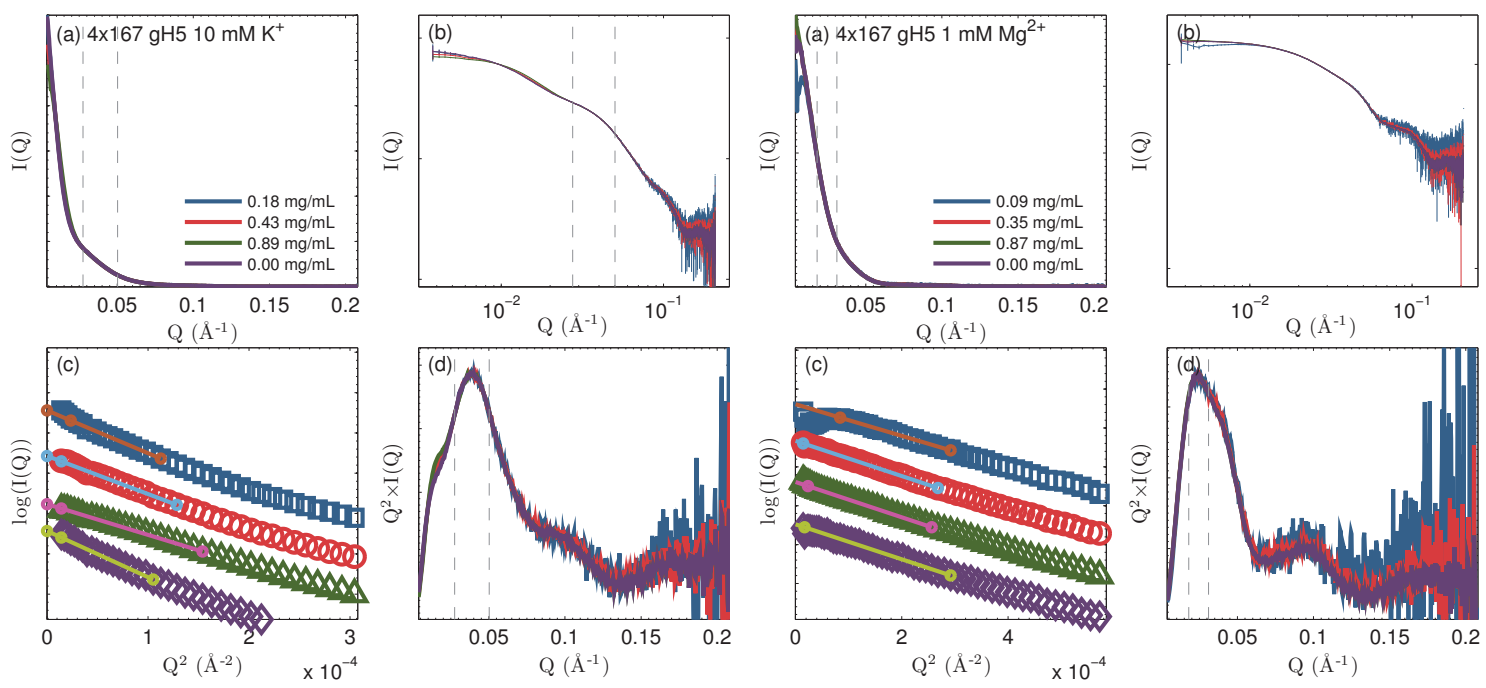

(i) $10 \mathrm{mM} \mathrm{K}^{+}$

(ii) $1 \mathrm{mM} \mathrm{Mg}^{2+}$

Figure 24. Reduced SAXS Profiles for $4 \times \mathbf{1 6 7}$ gH5 Arrays: (a)-(d) as in figs. 21-23, namely: (a)-(b) $I(Q)$ plots respectively on a linear and logarithmic scale. (c) Guinier plot with $R_{g}$ fits from the lowest usable $Q$-point up to $Q_{\max }=1.3 / R_{g}$. (d) Kratky plot, emphasizing the low- $Q$ features. The dashed vertical lines indicate the $Q$-range used for scaling the data.

\section{Observations}

Comparing the $R_{g}$ calculated from the Guinier region of each $I(Q)$, fig. 25, we observe interparticle interactions which match expectations based on the salt concentrations. An increase in $R_{g}$ as the sample concentration decreases indicates interparticle repulsion, seen for all arrays in $10 \mathrm{mM} \mathrm{K}^{+}$. A decrease in $R_{g}$ as the sample concentration decreases indicates interparticle attraction, seen for all arrays in $200 \mathrm{mM} \mathrm{K}^{+}$and $1 \mathrm{mM} \mathrm{Mg}^{2+}$. Arrays at intermediate salt concentrations, 50-100 $\mathrm{mM} \mathrm{K}^{+}$, show either a small amount of repulsion or negligible change for decreasing concentrations. These salt-induced interactions are a direct consequence of NCP electrostatics, for which a high concentrations of monovalent $\mathrm{K}^{+}$ions and even a low concentration of divalent $\mathrm{Mg}^{2+}$ ions will screen NCP repulsion and eventually lead to attraction. These trends match those seen with the mononucleosomes discussed in Chapter 2.

The zero concentration $R_{g}$ values for $3 \times 167$ arrays show that as the concentration 

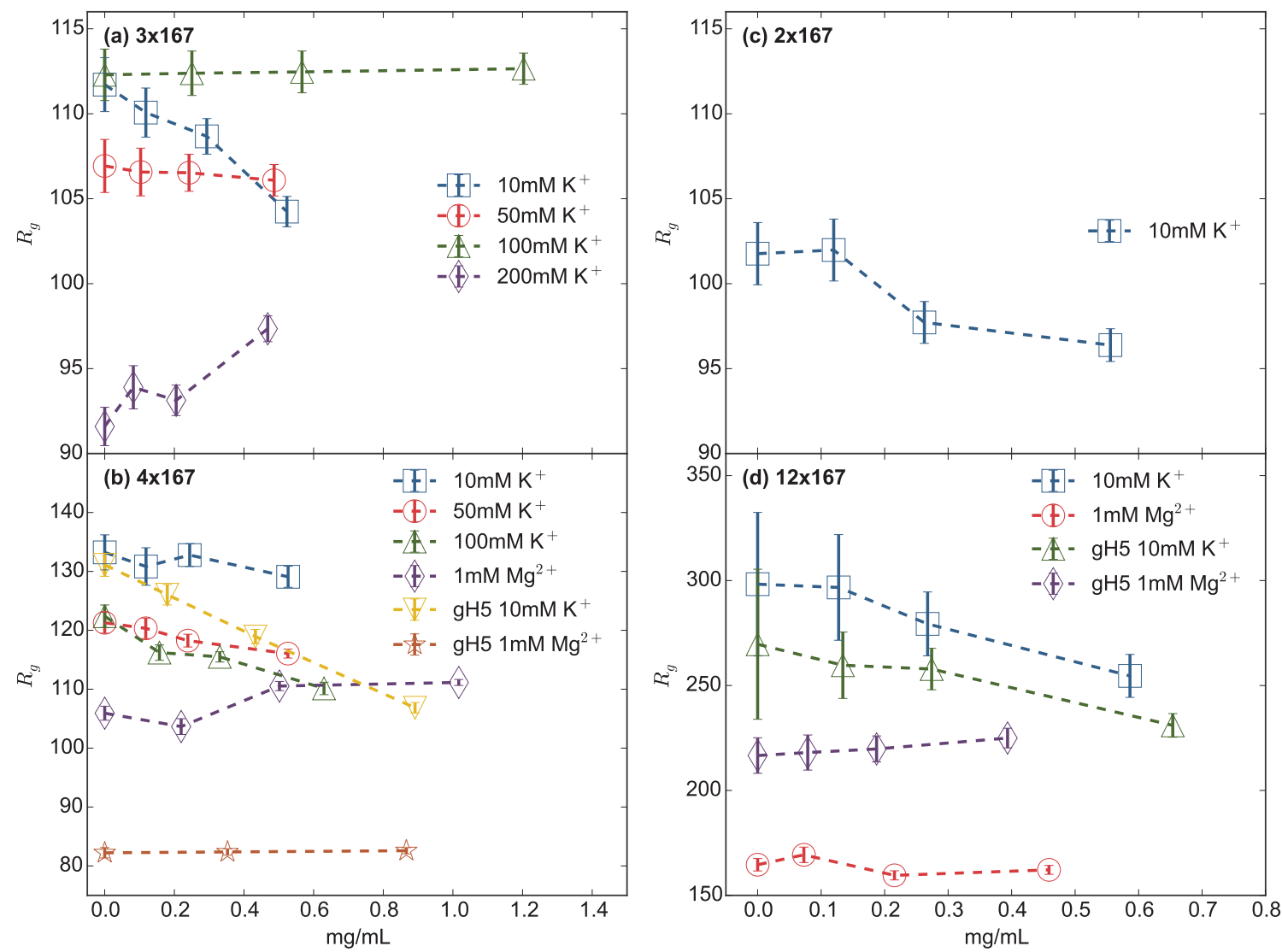

Figure 25. Comparison of the Nucleosome Array Guinier Fit Results: As the concentration increases, an increase in $R_{g}$ indicates interparticle attraction while a decrease in $R_{g}$ indicates interparticle repulsion.

of $\mathrm{K}^{+}$ions increase the array becomes more compact, as expected from nucleosome electrostatics. The one exception to this trend is that the $R_{g}$ values for $3 \times 167$ in 10 and $100 \mathrm{mM} \mathrm{K}^{+}$are essentially the same. The cause for this unexpected behavior is not clear but may indicate a problem in the low- $Q$ region of the $3 \times 167100 \mathrm{mM} \mathrm{K} \mathrm{K}^{+}$ data, e.g., interparticle interactions may still be present for this sample.

A comparison of the $3 \times 167$ form factors, fig. 26, shows a notable similarity between all ionic conditions. In particular, at all $\mathrm{K}^{+}$concentrations, the form factor is essentially identical between $0.1 \AA^{-1}<Q<0.2 \AA^{-1}$. This $Q$-region encodes information regarding structural features between $60 \AA>D>30 \AA$, or the size of the internal structure of the nucleosome. To better identify differences, we scaled each curve using this $Q$-range. 

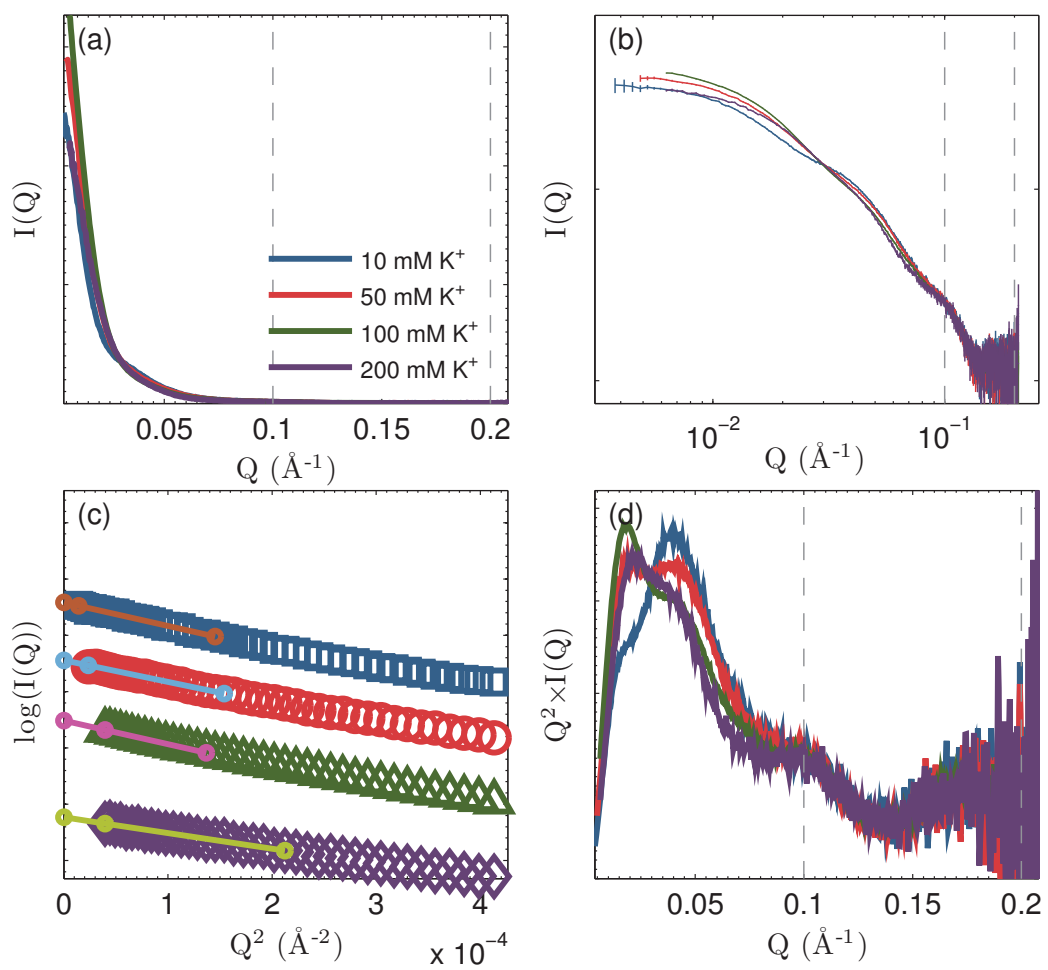

Figure 26. Form Factors of the $\mathbf{3} \times \mathbf{1 6 7}$ Arrays: (a)-(d) as in figs. 21-24, namely: (a)-(b) $I(Q)$ plots respectively on a linear and logarithmic scale. (c) Guinier plot with $R_{g}$ fits from the lowest usable $Q$-point up to $Q_{\max }=1.3 / R_{g}$. (d) Kratky plot, emphasizing the low- $Q$ features. Comparing the $3 \times 167$ form factors, we see in the logarithmic plot, and the Kratky plot, a strong similarity in the $Q$-range encoding the internal structure of the nucleosome, $0.1 \AA^{-1}<Q<0.2 \AA^{-1}$ (dashed vertical lines). Matching this $Q$-range for improved comparison, we recognize that as ionic screening increases, the Kratky peak at $0.040 \AA^{-1}$ becomes less prominent compared to the Kratky peak at $0.018 \AA^{-1}$.

Examining the Kratky plot, (d), which accentuates shape features in the mid-high $Q$-range, we see 2 distinct peaks at $0.040 \AA^{-1}$ and $0.018 \AA^{-1}$. These two peaks characterize a multidomain structure and correspond to structural features at $\sim 160 \AA$ and $\sim 350 \AA$ respectively. At $10 \mathrm{mM} \mathrm{K}^{+}$, the $Q=0.040 \AA^{-1}$ peak dominates compared to the $0.018 \AA^{-1}$ peak, appearing only as a shoulder. As nucleosomes connected by $20 \mathrm{bp}$ of straight linker DNA have a center-to-center distance of $\sim 160 \AA$, we interpret this $Q=0.018 \AA^{-1}$ Kratky peak to indicate the distances between adjacent nucleosomes is relatively conserved under low ionic screening. In other words, this corresponds to 
a situation where the distances between nucleosomes 1-2 and 2-3, are relatively conserved but that the distance between nucleosomes 1-3 is more varied. This matches the expectation that under low ionic screening nucleosome electrostatics will lead to an extended structure. For 100 and $200 \mathrm{mM} \mathrm{K}^{+}$, the situation is reversed with a much weaker peak at $0.040 \AA^{-1}$, indicating a lack of ordering at $\sim 160 \AA$. This points toward a less consistent distance between adjacent nucleosomes, i.e., more disordered structures. At $50 \mathrm{mM} \mathrm{K}{ }^{+}$, the two peaks show comparable intensities indicating a state of transition with some persistent ordering at $\sim 160 \AA$. Alternatively, the intermediate state at $50 \mathrm{mM} \mathrm{K}{ }^{+}$could indicate a mix of two structure populations in the solution ensemble, one similar to the $10 \mathrm{mM} \mathrm{K}^{+}$structures and the other similar to $100-200 \mathrm{~K}^{+}$structures.

From each $I(Q)$, we calculate $P(r)$ as discussed in Chapter 1 using GNOM [107]. In fig. 27, and also figs. 29 and 33 for the other constructs, panel (a) shows the error bars from the original $I(Q)$ overlain on the back transform, $I^{\prime}(Q)=\mathcal{F}^{-1}(\mathcal{F}((I(Q)))$, panel (b) shows the transformation $P(r)=\mathcal{F}(I(Q))$ with the total area under the
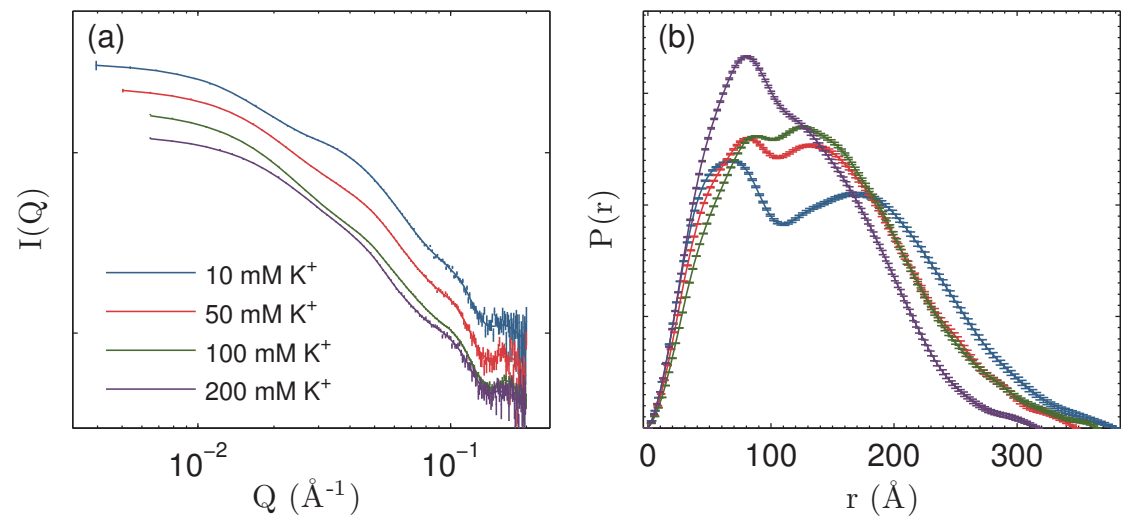

Figure 27. Pair-Distance Distributions from the $3 \times 167$ Arrays: (a) the error bars from the experimental $I(Q)$ overlain on the back transform $I^{\prime}(Q)=$ $\mathcal{F}^{-1}(\mathcal{F}(I(Q)))$. (b) the transformation $P(r)=\mathcal{F}(I(Q))$. As ionic screening increases, the distribution changes correspond to more compact structures with less order, respectively indicated by the decreased probability at large distances and the lack of clearly defined peaks. 
curve normalized to 1. The quality of the match in panel (a) of figs. 27, 29 and 33 indicates how much information about the system was contained outside of the measurement range, $Q<Q_{\min }$ and $Q>Q_{\max }$, and the validity of the selection of $D_{\max }$. For a well-behaved sample and a properly chosen $D_{\max }$, the original $I(Q)$ and the reverse transform from $P(r), I^{\prime}(Q)$, typically match for all measured $Q$-values. Another indicator of a properly chosen $D_{\max }$ is the lack of ripples in $P(r)$, particularly as $r$ approaches $D_{\max }$.

For the $3 \times 167 P(r)$ functions, fig. 27 , as salt increases we see two distinct peaks merge to a single peak with a shoulder. The two peaks for arrays in $10 \mathrm{mM} \mathrm{K}^{+}$, result from a multidomain structure and correspond to the distances within and between domains. The peak at small dimensions, $r \approx 67 \AA$, matches the single peak $P(r)$ from the nucleosome 1KX5 model [1] (not shown) and therefore originates from intra-nucleosome distances. The larger distance peak at $\sim 168 \AA$ corresponds well with the Kratky peak at $Q=0.040 \AA^{-1}, D=160 \AA$ which we attributed to the distance between nucleosomes. For increased $\left[\mathrm{K}^{+}\right]$, the merging of the peaks at lower distances clearly shows compaction and decreased order but it is difficult to make further conclusions regarding the details of what is occurring.

Comparing the $4 \times 167$ arrays in different ionic conditions, fig. 28, we see much of the same form factors changes as for the $3 \times 167$ arrays. The scattering from all conditions agree at $Q>0.1 \AA^{-1}$, with the $1 \mathrm{mM} \mathrm{Mg}^{2+}$ form factor showing some minor deviations. In the Kratky plot, (d), we again see the same distinct peaks at $0.040 \AA^{-1}$ and $0.018 \AA^{-1}$. As for the $3 \times 167$ arrays, the $0.040 \AA^{-1}$ peak dominates at $10 \mathrm{mM} \mathrm{K}^{+}$, while $50 \mathrm{mM} \mathrm{K}^{+}$produces an intermediate or transitional state, and the $0.018 \AA^{-1}$ peak dominates at $100 \mathrm{mM} \mathrm{K}^{+}$and also $1 \mathrm{mM} \mathrm{Mg}^{2+}$. The agreement between the $1 \mathrm{mM} \mathrm{Mg}^{2+}$ and $100 \mathrm{mM} \mathrm{K}^{+}$, despite the significant difference in ion concentration 1:100, results from the stronger screening effect of the divalent $\mathrm{Mg}^{2+}$; the screening by $\mathrm{Mg}^{2+}$ ions is so pronounced that arrays show similar behavior even 

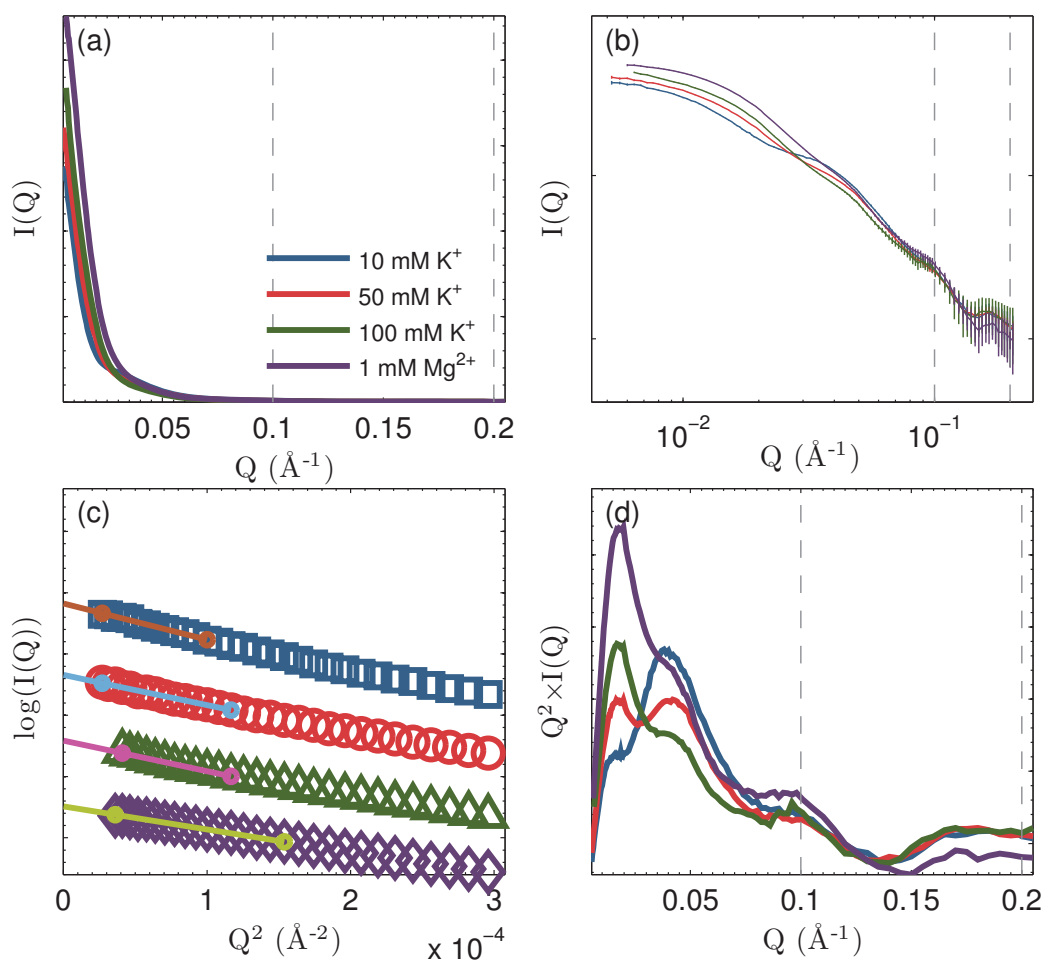

Figure 28. Form Factors of the $\mathbf{4} \times \mathbf{1 6 7}$ Arrays: (a)-(d) as in figs. 21-24 and 26, namely: (a)-(b) $I(Q)$ plots respectively on a linear and logarithmic scale. (c) Guinier plot with $R_{g}$ fits from the lowest usable $Q$-point up to $Q_{\max }=1.3 / R_{g}$. (d) Kratky plot, emphasizing the low- $Q$ features. Comparing the $4 \times 167$ form factors, we see in the logarithmic plot, and the Kratky plot, a strong similarity in the $Q$-range encoding the internal structure of the nucleosome, $0.1 \AA^{-1}<Q<0.2 \AA^{-1}$ (dashed vertical lines). Matching this $Q$-range for improved comparison, we recognize that as ionic screening increases, the Kratky peak at $0.040 \AA^{-1}$ becomes less prominent compared to the Kratky peak at $0.018 \AA^{-1}$. This resembles the changes seen for the $3 \times 167$ arrays in fig. 26 .

when the total charge of the $\mathrm{Mg}^{2+}$ cations is $1 / 50^{\text {th }}$ that of the $\mathrm{K}^{+}$cations. The similarities to the $3 \times 167$ Kratky plots indicate consistent nucleosome conformations and conformational changes in varied ionic conditions, despite the addition of a $4^{\text {th }}$ nucleosome. It is somewhat surprising that the position of the first Kratky peak in high ionic screening for $3 \times 167$ and $4 \times 167$ arrays are both centered as $Q=0.004 \AA^{-1}$. This consistency implies the high salt ordering does not originate from the overall size of the macromolecule though it remains unclear what consistent structure features leads to this Kratky peak. 

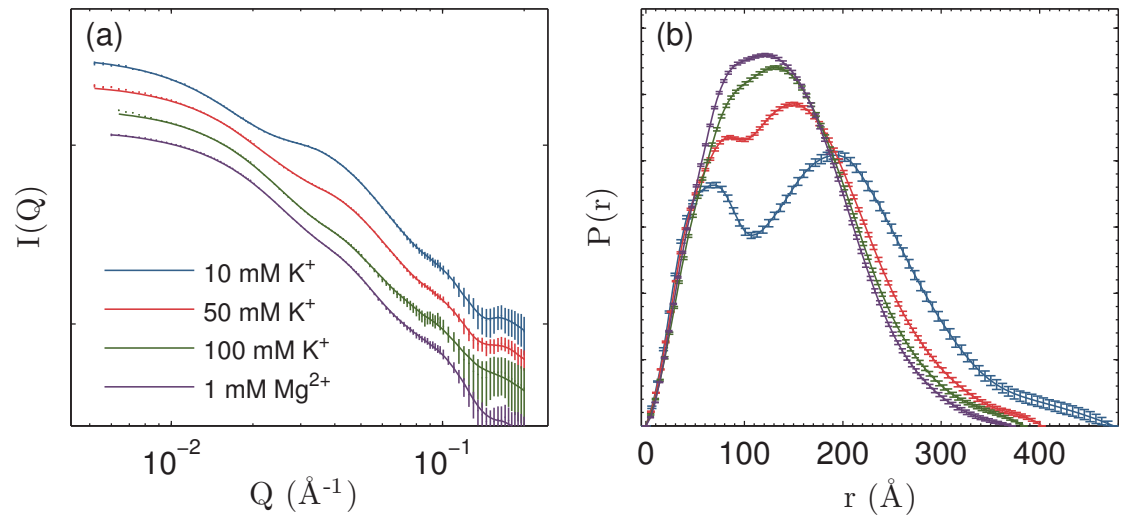

Figure 29. Pair-Distance Distributions from the $\mathbf{4} \times \mathbf{1 6 7}$ Arrays: (a)-(b) as in fig. 27, namely: (a) the error bars from the experimental $I(Q)$ overlain on the back transform $I^{\prime}(Q)=\mathcal{F}^{-1}(\mathcal{F}(I(Q)))$. (b) the transformation $P(r)=\mathcal{F}(I(Q))$. Similar to the change seen in $3 \times 167$ arrays, as ionic screening increases, the distribution changes correspond to more compact structures with less order, respectively indicated by the decreased probability at large distances and the lack of clearly defined peaks.

For the $4 \times 167 P(r)$ plots, fig. 29, we again see 2 peaks for arrays in $10 \mathrm{mM}$ $\mathrm{K}^{+}$which merge to one peak under increased ionic screening. Unlike $3 \times 167$ arrays, by $100 \mathrm{mM} \mathrm{K}^{+}$the $4 \times 167 \mathrm{P}(r)$ does not show 2 distinct peaks but has merged to one broad peak. This change again indicates a transition from a globular extended structure at low ionic screening to a disorganize compact structure at high ionic screening.

To get a better idea of the change in scattering form factor resulting from arrays of different length, fig. 30 compares all arrays measured at $10 \mathrm{mM} \mathrm{K}^{+}$, (i), and $100 \mathrm{mM}$ $\mathrm{K}^{+}$, (ii). Again scaling to match at $Q \geq 0.1 \AA^{-1}$, in $10 \mathrm{mM} \mathrm{K}^{+}$, the form factors for 2 , 3,4 , and 12 nucleosomes show essentially no difference in shape above $Q>0.05 \AA^{-1}$ and all exhibit the strongest Kratky peak around $Q=0.040 \AA^{-1}$. At $100 \mathrm{mM} \mathrm{K}^{+}$, the form factors from $3 \times 167$ and $4 \times 167$ arrays again show strong similarities, though in this case the most visible differences are in the intermediate range of $0.016 \AA^{-1}<$ $Q<0.090 \AA^{-1}$ (we do have data for $2 \times 167$ and $12 \times 167$ arrays at $100 \mathrm{mM} \mathrm{K}^{+}$).

These similarities are also seen in the $P(r)$ distributions for these data, fig. 31. At 

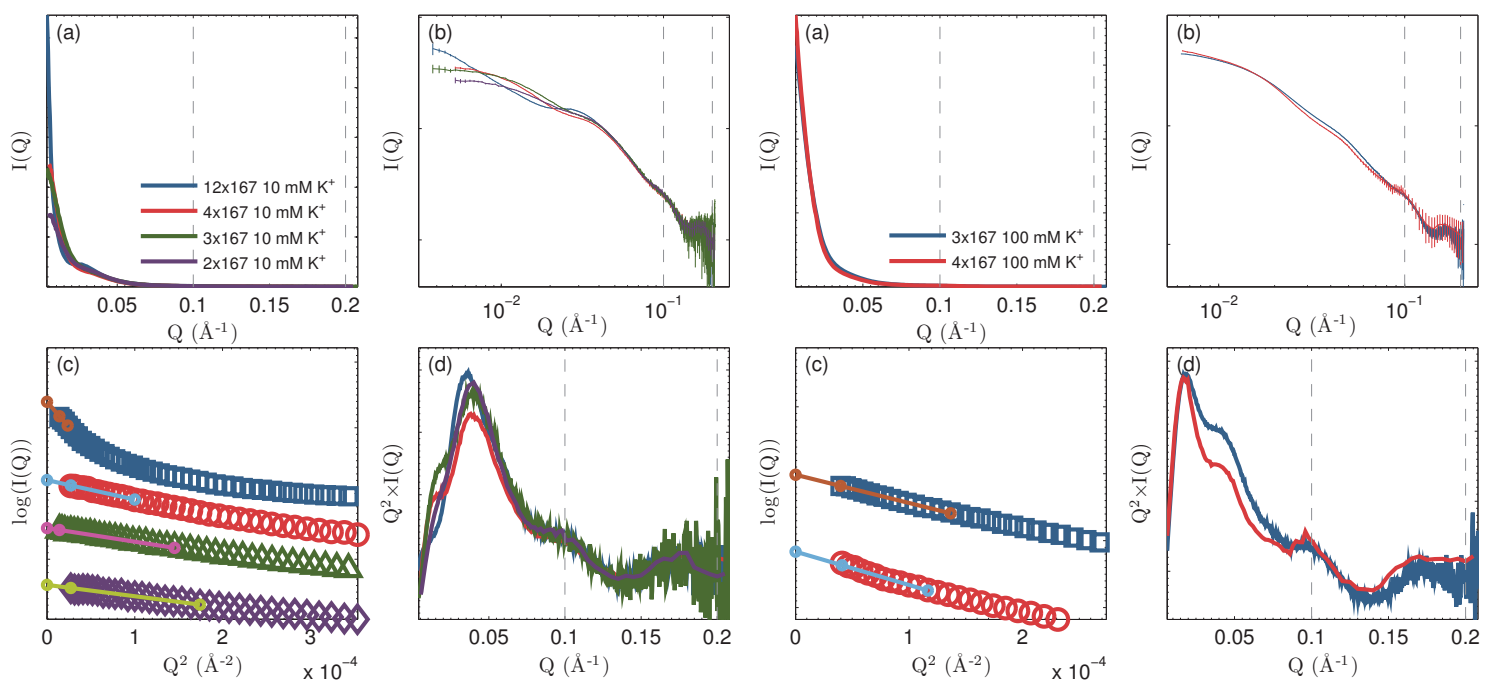

(i) $10 \mathrm{mM} \mathrm{K}^{+}$

(ii) $100 \mathrm{mM} \mathrm{K}^{+}$

Figure 30. Form Factors for Nucleosome Arrays of Varied Length: (a)-(d) as in figs. 21-24, 26 and 28, namely: (a) $-($ b) $I(Q)$ plots respectively on a linear and logarithmic scale. (c) Guinier plot with $R_{g}$ fits from the lowest usable $Q$-point up to $Q_{\max }=1.3 / R_{g}$. (d) Kratky plot, emphasizing the low- $Q$ features. Again matching at $Q \geq 0.1 \AA^{-1}$ (vertical dashed lines) for improved comparison, in $10 \mathrm{mM} \mathrm{K}^{+}$, (i), we see a strong similarity for $Q \geq 0.05 \AA^{-1}$ in the form factors from arrays with 2 , 3,4 , and $12 \mathrm{NCPs}$. In $100 \mathrm{mM} \mathrm{K}^{+}$, (ii), the arrays with 3 and 4 NCPs differ most prominently in the range $0.016 \AA^{-1}<Q<0.090 \AA^{-1}$.

$10 \mathrm{mM} \mathrm{K}^{+},(\mathrm{a})$, all array lengths show the single nucleosome peak and a second peak corresponding to the distance between nucleosomes. The relative ratio between these peaks follows the trend that as the number of nucleosomes increase, the number of inter-nucleosome distances also increases making the second peak larger. In $100 \mathrm{mM}$ $\mathrm{K}^{+}$, (b), the $P(r)$ from $3 \times 167$ and $4 \times 167$ arrays are strikingly similar, the most significant difference being the single nucleosome peak still slightly visible for the $3 \times 167$; this difference is a consequence of the $3 \times 167$ array having fewer inter-nucleosome distances compared to the $4 \times 167$ array.

The uniformity in conformations across arrays with a varied number of nucleosomes affirms that salt-induced conformation changes are independent of the number of nucleosomes. Particularly for low ionic screening, we see that structural features below the size of $\sim D<160 \AA$ are conserved as the number of nucleosomes increases. 

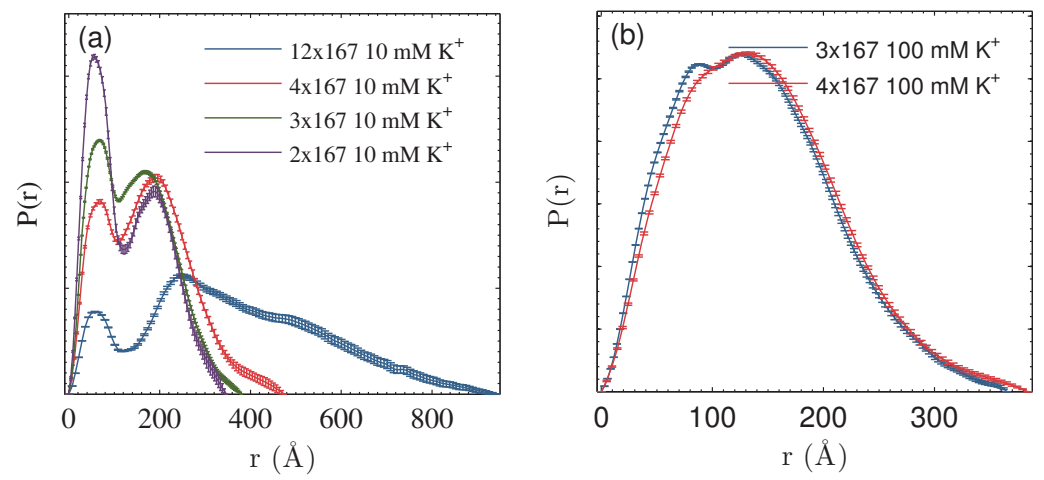

Figure 31. Pair-Distance Distributions for Nucleosome Arrays of Varied Length: Comparing the $P(r)$ from arrays of varied length in $10 \mathrm{mM} \mathrm{K}^{+}$, (a), we see a bimodal distribution, representing a globular extended structure. The first peak, at $\sim 60 \AA$, corresponds to the distribution of a single nucleosome. The second peak represents the inter-nucleosome distances. In $100 \mathrm{mM} \mathrm{K}^{+}$, (b), the most distinguishing difference between the $P(r)$ from $3 \times 167$ and $4 \times 167$ arrays is the difference at $90 \AA$; what appears as a peak in the $3 \times 167$ arrays is only visible as a shoulder in the $4 \times 167$ arrays. This can be attributed to the $3 \times 167$ array having fewer internucleosome distances, therefore the single nucleosome peak is stronger in relation to the inter-nucleosome peak.

This consistency confirms the expectations that the NCP unit of the array remains fully intact and that their negative charge repels other NCPs in the array, independent of the array length. In the more compact structures, caused by high ionic screening, we would expect the structural difference to originate from interactions between nextnearest-neighbors, i.e., $\mathrm{NCP}_{1}$ with $\mathrm{NCP}_{3}$ or $\mathrm{NCP}_{2}$ with $\mathrm{NCP} 4$, as the $4 \times 167$ array would have twice as many of these interactions compared to the $3 \times 167$ array. Qualitatively, it is not clear how this factors into the comparison for higher ionic screening, nor is it clear why the most prominent differences are in this intermediate Q-region. For both ionic conditions, more in-depth modeling is required to gain further insight into the structural changes resulting from increased number of NCP repeats.

Results from $4 \times 167$ arrays with added gH5 linker protein, fig. 32, demonstrate a conformation transition resembling that of arrays without the gH5 linker though the resulting peak in high ionic screening, $1 \mathrm{mM} \mathrm{Mg}^{2+}$, seems to be a combination of 

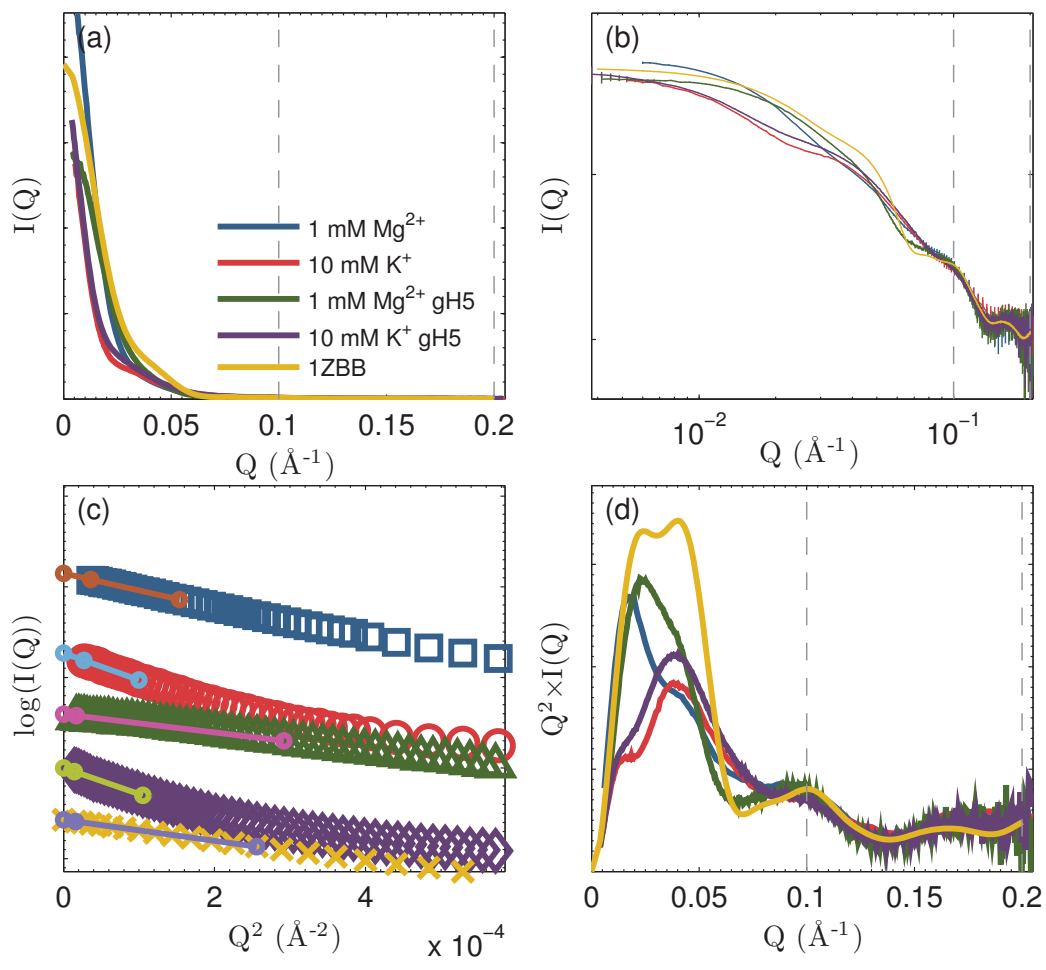

Figure 32. Effect of gH5 on the $\mathbf{4} \times \mathbf{1 6 7}$ Form Factors: (a)-(d) as in figs. $21-24$, 26, 28 and 30, namely: (a)-(b) $I(Q)$ plots respectively on a linear and logarithmic scale. (c) Guinier plot with $R_{g}$ fits from the lowest usable $Q$-point up to $Q_{\max }=$ $1.3 / R_{g}$. (d) Kratky plot, emphasizing the low- $Q$ features. Again matching at $Q \geq$ $0.1 \AA^{-1}$ (vertical dashed lines) for improved comparison, arrays with and without gH5 show minor differences in $10 \mathrm{mM} \mathrm{K}^{+}$but drastic differences in $1 \mathrm{mM} \mathrm{Mg}^{2+}$. The 4x167 array with gH5 in $1 \mathrm{mM} \mathrm{Mg}^{2+}$ shows the closest match to the tetranucleosome crystal structure (PDB ID: 1ZBB) [2].

the $0.040 \AA^{-1}$ and $0.018 \AA^{-1}$ peaks. The fact that in the $1 \mathrm{mM} \mathrm{Mg}^{2+}$ condition the $0.040 \AA^{-1}$ peak in the Kratky plot is not dominated by the $0.018 \AA^{-1}$ peak indicates a preservation of ordering at $160 \AA$. This preservation of order around $160 \AA$ likely results from the distance between neighboring nucleosomes being maintained for arrays with gH5. As this is not the case for arrays without gH5, this difference likely indicates increased DNA unwrapping or DNA breathing from the NCPs without gH5.

Compared to the other $4 \times 167$ data, the form factor from $4 \times 167$ with $\mathrm{gH} 5$ in $1 \mathrm{mM} \mathrm{Mg}^{2+}$ most closely resembles the theoretical form factor, calculated using FoXS [108], from the tetranucleosome crystal model (PDB ID: 1ZBB) [2]. Considering the 
binding site of $\mathrm{gH} 5$ provides insight into possible origins for this similarity and also the preservation of the $160 \AA$ distance between nucleosomes. The gH5 linker histone binds to the nucleosome dyad between the linker DNA, acting somewhat like a hairpin between these two DNA linkers. This binding inherently produces added structural support that inhibits DNA unwrapping and DNA breathing. The similarity to the 1ZBB form factor indicates that the addition of gH5 produces regular packing of nucleosomes, similar to what is seen in crystal.

The similarity is even more obvious when comparing the $P(r)$ distributions for the $4 \times 167$ array with $\mathrm{gH} 5$ in $1 \mathrm{mM} \mathrm{Mg}^{2+}$ and the $1 \mathrm{ZBB}$ model, fig. 33 . While the distribution from $4 \times 167$ arrays with and without $\mathrm{gH} 5$ show little difference in $10 \mathrm{mM}$ $\mathrm{K}^{+}$, the distributions from the same arrays in $1 \mathrm{mM} \mathrm{Mg}^{2+}$ are drastically different. In particular, the structure of arrays with $\mathrm{gH} 5$ in $1 \mathrm{mM} \mathrm{Mg}^{2+}$ are much more compact, indicated by the low probability at higher distances (also already apparent from the $R_{g}$ ), and much more ordered, indicated by the sharp rather than broad peak. Of all the $4 \times 167$ arrays, the $4 \times 167$ with gH5 in $1 \mathrm{mM} \mathrm{Mg}^{2+}$ most closely resembles the
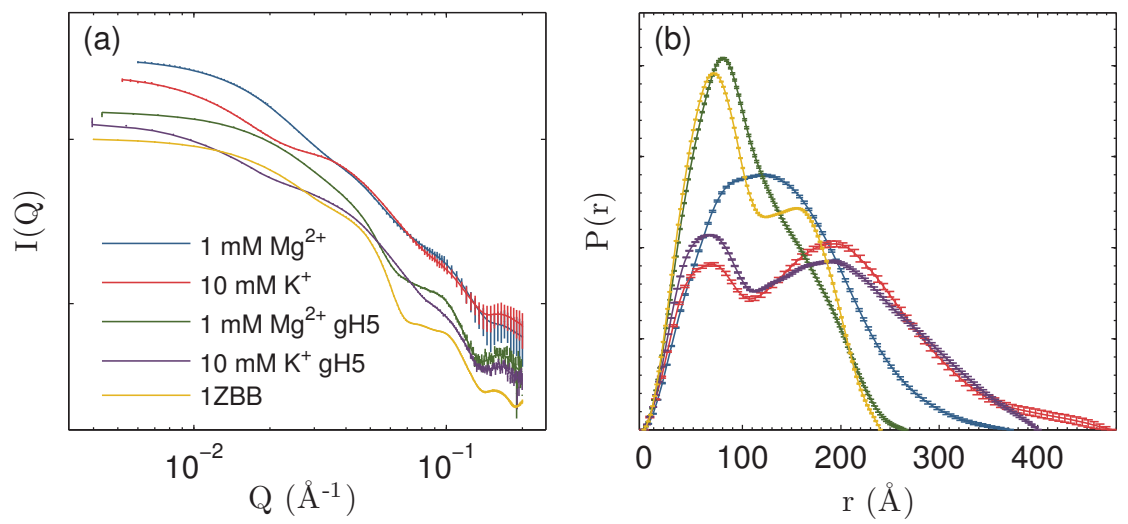

Figure 33. Pair-Distance Distributions from the $\mathbf{4} \times \mathbf{1 6 7}$ gH5 Arrays (a)-(b) as in figs. 27 and 29, namely: (a) the error bars from the experimental $I(Q)$ overlain on the back transform $I^{\prime}(Q)=\mathcal{F}^{-1}(\mathcal{F}(I(Q)))$. (b) the transformation $P(r)=\mathcal{F}(I(Q))$. Similar to the differences seen in the form factors, gH5 addition has little affect in $10 \mathrm{mM} \mathrm{K}^{+}$but a major affect in $1 \mathrm{mM} \mathrm{Mg}^{2+}$. The $P(r)$ accentuates the resemblance between the $4 \times 167$ array with $\mathrm{gH} 5$ in $1 \mathrm{mM} \mathrm{Mg}^{2+}$ and the tetranucleosome crystal structure (PDB ID: 1ZBB) [2]. 
1ZBB $P(r)$. Comparing these, the 1ZBB $P(r)$ shows the same sharp peak located a few Åsmaller, $r=71 \AA$ instead of $r=80 \AA$ but also has a second peak at $155 \AA$ which for the $4 \times 167$ gH5 array is expressed more as a shoulder.

\section{User-Directed Rigid Body Modeling of $4 \times 167$ gH5 Arrays}

The similarity between $4 \times 167 \mathrm{gH} 5$ arrays in $1 \mathrm{mM} \mathrm{Mg}^{2+}$ and the $1 \mathrm{ZBB}$ model of the $4 \times 167$ crystal structure [2] motivated using this atomic model as starting structure for developing model structures which better match the experimental results. The first step to this modeling was to create a starting model which matched the molecular composition of the array studied experimentally. Compared to our $4 \times 167$ array, the 1ZBB model does not contain many of the histone protein tails, it has an extra DNA $\mathrm{bp}$, and the DNA sequence differs significantly.

To resolve these differences between the 1ZBB model and our $4 \times 167$ array, we

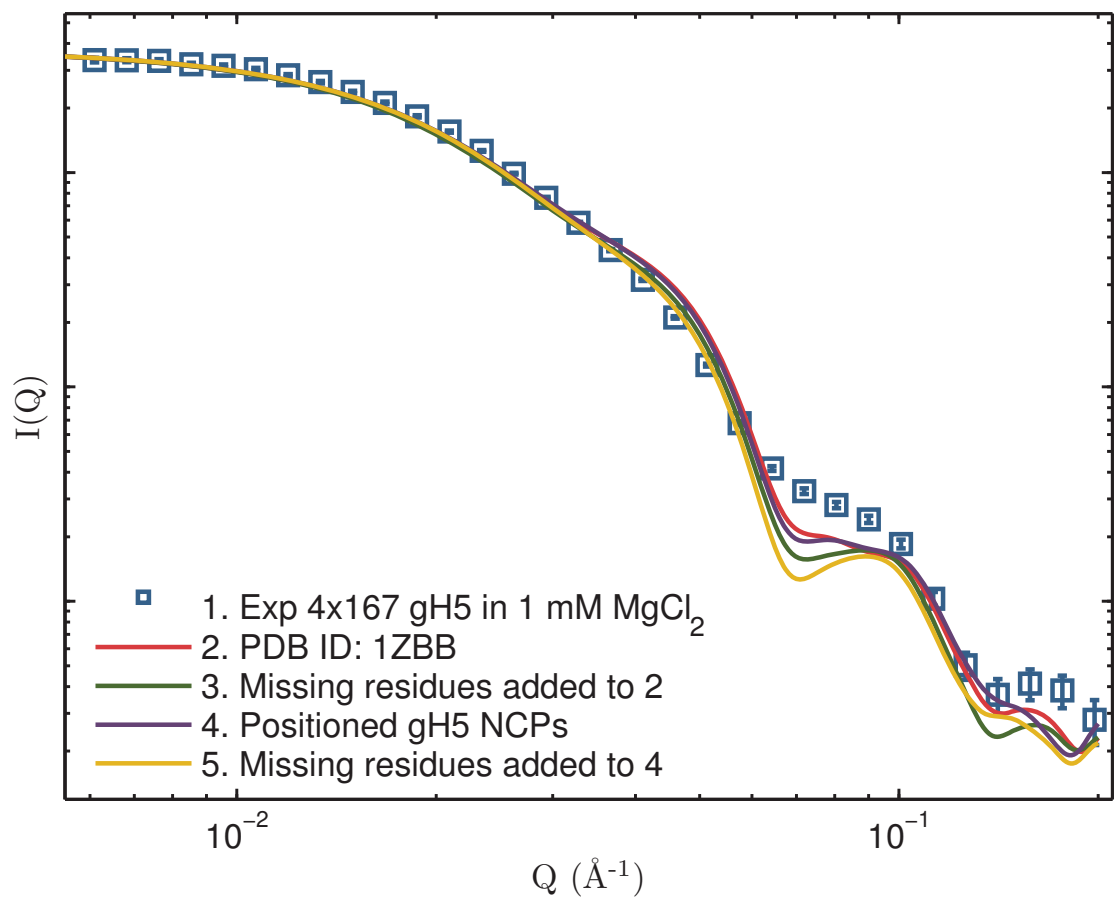

Figure 34. Form Factor Comparison: Comparison of the experimental $4 \times 167$ $\mathrm{gH} 5$ array in $1 \mathrm{mM} \mathrm{Mg}^{2+}$ with form factors calculated from various model structures. 
created a model which contains every residue from the experimental construct by replacing the histone proteins with nearly complete versions, deleting incorrect DNA atoms, then inserting any missing atoms using PSFGEN, part of the NAMD package [3]. These corrections produced an atomic model that matched the experimental $4 \times 167$ array, without gH5 protein. Figure 34 compares the experimental form factor together with the calculated form factors from the original and corrected 1ZBB model, respectively curves $1-3$. Model data is scaled to match the experimental $I(0)$ as this represents the molecular weight of the scattering particle, discussed further in Chapter 5. The addition of the missing residues to the 1ZBB model produced the most pronounced changes around the poorly matched $\operatorname{dip}$ at $Q \simeq 0.014 \AA^{-1}$.

In order to compare the experimental data with geometrically modified versions of the 1ZBB structure with the gH5 proteins, we developed an algorithm which generates model nucleosome array structures using copies of a gH5 NCP model together with linker DNA fragments. We created a gH5 NCP model from the 1KX5 NCP model [1] by changing the DNA sequence to match the W601 sequence, again using PSFGEN, then aligned an atomic model of the gH5 protein to the dyad position using Python scripts modified from SASSIE [109]. We determined the relative position and orientation of each $\mathrm{NCP}$ in the $1 \mathrm{ZBB}$ model by fitting a cylinder to the $\mathrm{C} 1^{\prime}$ atoms of the DNA backbone. ${ }^{7}$ From these NCP coordinates and orientations, we calculated the translations and rotations required to transform one NCP to the next NCP along the DNA chain. Applying these transformation parameters to the modified 1KX5 NCP model with a gH5 linker, we created a version of the 1ZBB with gH5 linker histones, lacking 3 bp of linker DNA between each NCP and also missing the extra DNA on the first and last NCP. The calculated form factor for this segmented array model with gH5 is curve 4 in fig. 34 .

To include the missing DNA, we added $3 \mathrm{bp}$ of DNA between each NCP and 14

\footnotetext{
${ }^{7}$ Figure 49 illustrates a DNA molecule and labels the $\mathrm{C} 1^{\prime}$ and $\mathrm{C} 2{ }^{\prime}$ atoms.
} 
bp of DNA before and after the first and last NCP. We inserted the missing $3 \mathrm{bp}$ of linker DNA between each nucleosome by aligning a $7 \mathrm{bp}$ fragment with the $\mathrm{C} 1^{\prime}$ atoms of the last two DNA residues of the $3^{\prime}$ DNA tail on the first $\mathrm{NCP}$ and the $\mathrm{C}^{\prime}$ and $\mathrm{C} 2^{\prime}$ atoms of the second residue on the $5^{\prime} \mathrm{DNA}$ tail of the second $\mathrm{NCP}$. Performing the alignment of the linker DNA using two atoms on each end but with an asymmetric atomic selection provided a directional yet evenly weighted basis for inserting the missing DNA bp. After alignment, the middle $3 \mathrm{bp}$ of the $7 \mathrm{bp}$ fragment were combined into the array structure, removing the 4 used for alignment purposes. On the first and last NCP, we attached 14 bp DNA end fragments, in a manner similar to the linker DNA, using an extra 3 bp of overlapping DNA. For these prefix and suffix DNA sections, we achieved directional placement of the 3 bp overlap by aligning to both the $\mathrm{C}^{\prime}$ and $\mathrm{C} 2^{\prime}$ atoms on the $5^{\prime} \mathrm{DNA}$ strand and only the $\mathrm{C}^{\prime}$ atoms on the $3^{\prime}$ DNA strand.

With these additions, the DNA sequence was a $99.4 \%$ match to the recombinant DNA. The remaining $0.6 \%$ difference resulted from a 4 bp sequence difference in the $4 \times 167$ DNA template immediately following the W601 DNA on the fourth NCP. The difference in sequence would amount to an imperceptible difference in molecular weight, and therefore would have no effect at low- $Q$. Further, such a small difference would produce a negligible difference in X-ray scattering as the difference in the electron distribution from 4 DNA bp would be trivial with respect to such a large molecule.

Comparing the form factors from the corrected 1ZBB model and the manually generated gH5 array model, fig. 34 curves 3 and 5, we attribute the slight differences to the addition of the gH5 proteins and differences in the position of the linker and end DNA fragments. Juxtaposed with the form factors from the models missing residues, fig. 34 curves 2 and 4, we see the significance of these residue additions and corrections for the resulting form factor. 


\section{Rigid Body Moves}

The form factors for the model structures and the $4 \times 167 \mathrm{gH} 5$ array in $1 \mathrm{mM} \mathrm{Mg}^{2+}$ differ most significantly at the dip around $Q=0.07 \AA^{-1}$. This difference corresponds to structural features roughly $90 \AA$ in size, or approximately the distance between stacked NCPs in the 1ZBB crystal structure. To generate better-matched models, starting from the 1ZBB configuration, we performed systematic rigid body moves of the NCPs in the $4 \times 167 \mathrm{gH} 5 \mathrm{NCP}$ model.

To facilitate these moves, we assigned a local coordinate system to each NCP and defined critical array axes and coordinates, illustrated in fig. 35, similar to those defined by Schalch et al. [2]. The two primary axes for each NCP are its cylinder axis

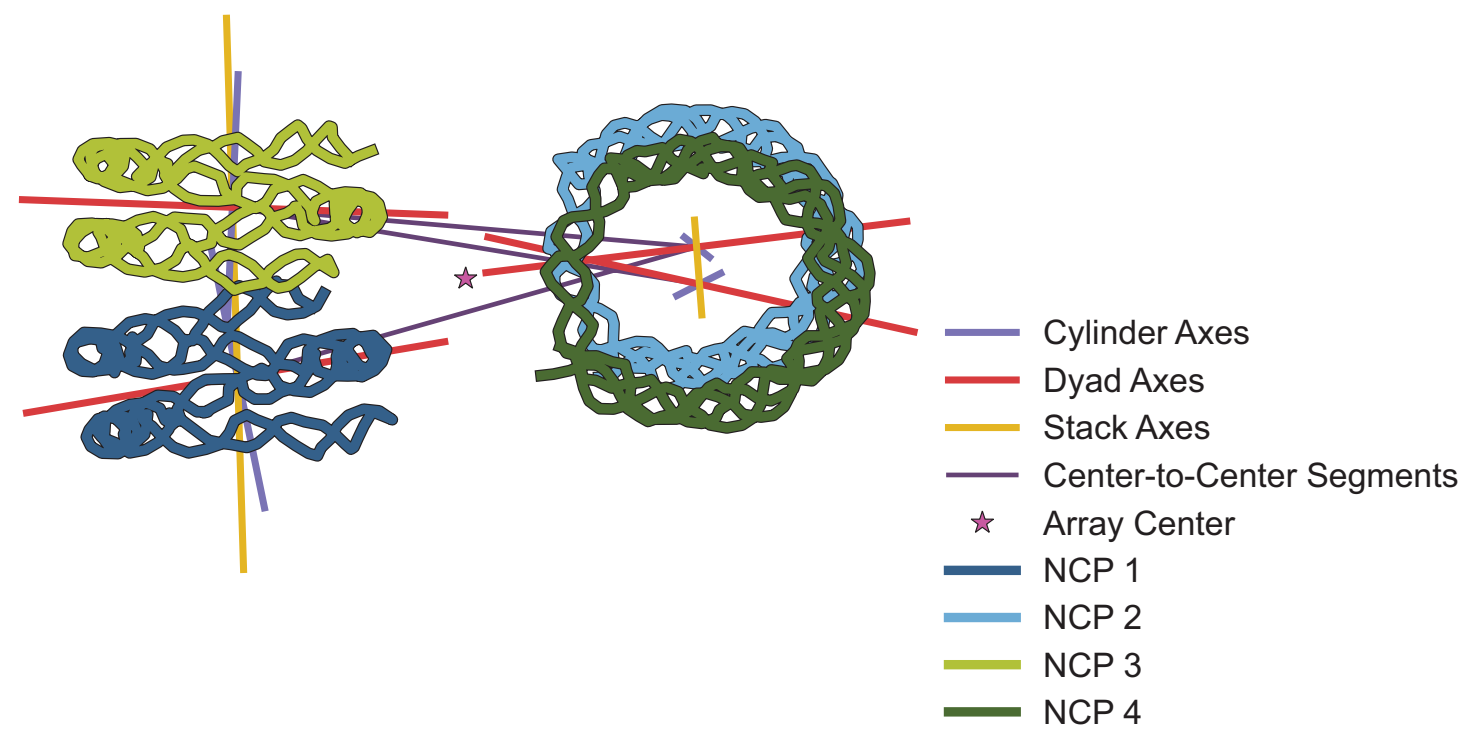

Figure 35. Geometric Definitions for the Tetranucleosome Array: Illustration of the geometry axes used to describe rigid body transformations of NCPs within the tetranucleosome array. Each cylinder axis is determined by fitting a cylinder to the $\mathrm{C}^{\prime}$ atoms of the NCP. The dyad axis orthogonally intersects the cylinder axis and passes through the center of the DNA bp located at the nucleosome dyad. The intersection of these two axes defines the center of the NCP. The line passing through the centers of stacked $\mathrm{NCPs}$, e.g., $\mathrm{NCP}_{1}$ and $\mathrm{NCP}_{3}$, defines the stack axis. The line segment between the centers of adjacent $\mathrm{NCPs}$, e.g., $\mathrm{NCP}_{1}$ and $\mathrm{NCP}_{2}$, defines the center-to-center segment. The array center is the midpoint between the four NCP centers. Each NCP is numbered sequentially from the $5^{\prime}$ end of the template DNA. 
and dyad axis. The cylinder axis is defined by fitting a cylinder to the $\mathrm{C}^{\prime}$ atoms of the W601 DNA in the NCP. The dyad axis is defined as the line orthogonally intersecting the cylinder axis and passing through the center of the DNA bp [110] at the nucleosome dyad. The intersection of the cylinder and dyad axes define the NCP center. The line segments connecting the center of successive NCPs along the DNA chain define the two opening angles of the array, e.g., the angle between the segments connecting $\mathrm{NCP}_{1}-\mathrm{NCP}_{2}$ and $\mathrm{NCP}_{2}-\mathrm{NCP}_{3}$ define $\psi_{123}$. The $\mathrm{NCP}$ stack axes are defined by the line segments passing through the centers of stacked NCPs, e.g., the line between the centers of $\mathrm{NCP}_{1}$ and $\mathrm{NCP}_{3}$. The center of the tetranucleosome array is defined by the midpoint between the four NCP centers. In addition to defining these axes, we number each NCP sequentially starting at the $5^{\prime}$ end of the template DNA.

We examined the changes in the form factors of three separate modifications, changing the opening angle between center-to-center segments, twisting NCPs about their dyad axis, and sliding stacked NCPs orthogonal to the stack axis. To determine the best-matched structures, we calculated the form factor for all model structures then quantified deviations between each model and experimental form factor using the SAXS analog of the crystallography $R$-factor [111], defined as,

$$
R=\frac{\sum_{i=1}^{N}\left|I_{e}\left(Q_{i}\right)-c_{\text {scale }} I_{m}\left(Q_{i}\right)\right|}{\sum_{i=1}^{N}\left|I_{e}\left(Q_{i}\right)\right|},
$$

where $I_{m}$ and $I_{e}$ are respectively the model and experimental form factors being compared, $N$ is the number of $Q$ points in the form factor, and $c_{\text {scale }}=I_{e}(0) / I_{m}(0)$ is the scale factor used to match the model to the experimental $I(0)$. Here we show the comparison of model structures to the $4 \times 167 \mathrm{gH} 5$ array in $1 \mathrm{mM} \mathrm{Mg}^{2+}$ and in $10 \mathrm{mM} \mathrm{K}^{+}$. Compared to the form factor of the starting structure, fig 34 curve 5, the 
form factor from $4 \times 167 \mathrm{gH} 5$ array in $1 \mathrm{mM} \mathrm{Mg}^{2+}$ and $10 \mathrm{mM} \mathrm{K}^{+}$respectively have $R$ values of 0.388 and 0.0381 .

\section{Opening Angle}

The initial opening angles between the NCPs in the 1ZBB configuration are $\psi_{123}=22.9^{\circ}$ and $\psi_{234}=23.0^{\circ}$ which respectively correspond to stack distances of $57.8 \AA$ and $58.2 \AA$. Figure 36 illustrates simultaneously incrementing $\psi_{123}$

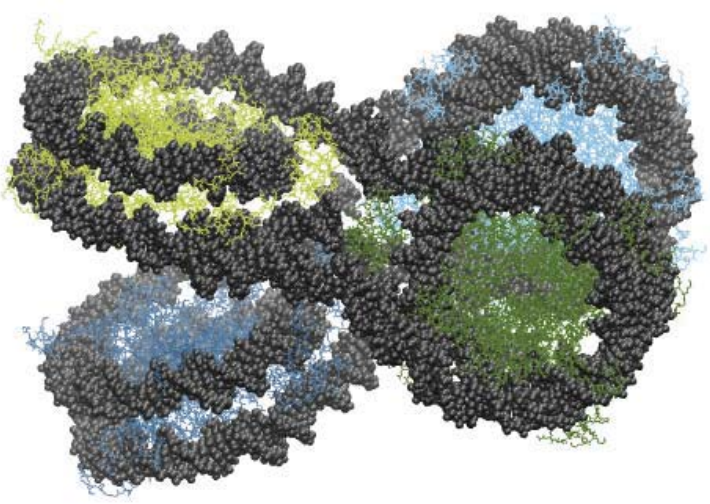
and $\psi_{234}$ between $\Delta \psi=-10^{\circ}, \ldots, 40^{\circ}$. The $R$ value for each of the resulting models compared to $10 \mathrm{mM} \mathrm{K}^{+}$and $1 \mathrm{mM} \mathrm{Mg}^{2+}$ is shown in fig. 37 .

For the $10 \mathrm{mM} \mathrm{K}^{+}$form factor, none of the explored opening angles produce an absolute minimum, though the model with the largest opening angle produced

Figure 36. NCP Opening Angle Rigid Body Moves: Simultaneously changing the opening angles between NCPs by $\Delta \psi=-10^{\circ}, \ldots, 45^{\circ}$. The NCP proteins are colored to match the DNA in fig. 35, namely: $\mathrm{NCP}_{1}-\mathrm{NCP}_{4}$ respectively dark blue, light blue, light green, dark green. (Animated in electronic version, $\Delta \psi=10^{\circ}$ is shown in the print version.)
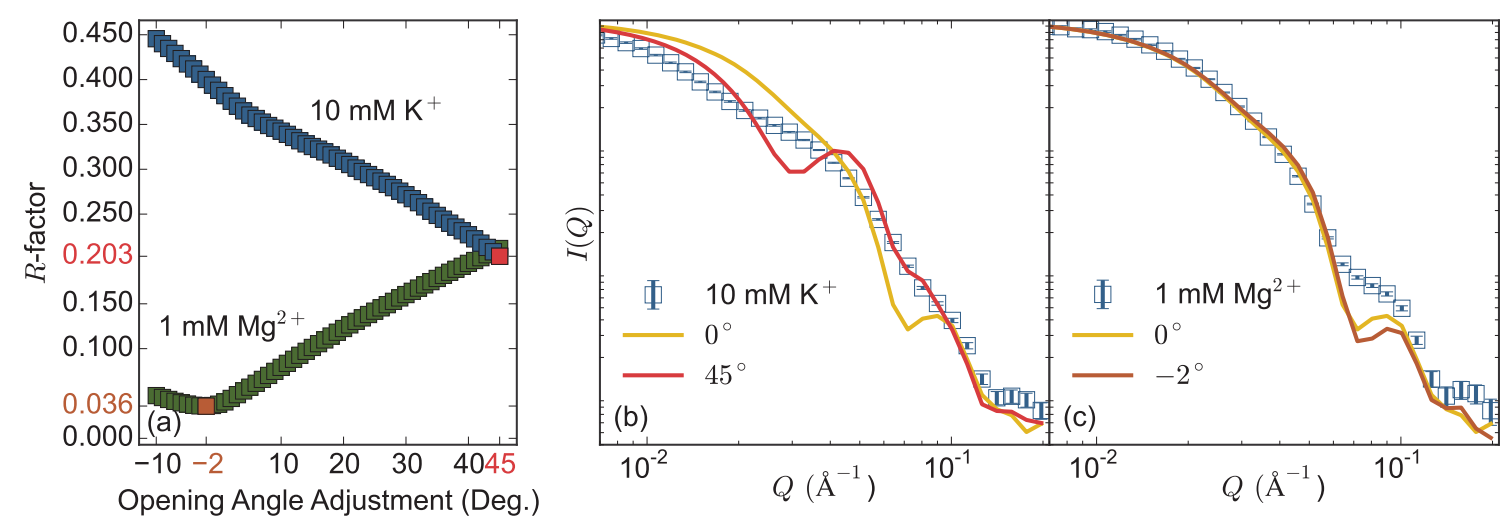

Figure 37. Best-Matched Models from Opening Angle Moves: The $R$-factor comparison, (a), and best-matched form factors, (b) and (c), for incremental adjustments of the opening angle between NCPs by $\Delta \psi=-10^{\circ}, \ldots, 45^{\circ}$. 
$R=0.203$ at the largest angle, $\Delta \psi=45^{\circ}$. This represents the NCPs being stacked at a distance of $\sim 105 \AA$. Even with the improved $R$, the shape of the experimental and model form factors still differ significantly, fig. 37(b). This inability to reproduce the form factor shape, most importantly in the low- $Q$ Guinier region, demonstrates the solution structure of the $4 \times 167 \mathrm{gH} 5$ array in $10 \mathrm{mM} \mathrm{K}^{+}$significantly differs from the compact $1 \mathrm{ZBB}$ structure.

For the $1 \mathrm{mM} \mathrm{Mg}^{2+}$, a minimum $R=0.036$ occurs when the opening angles are decreased by $\Delta \psi=-2^{\circ}$, corresponding to a decrease in the stack distances by $4.9 \AA$, to $52.9 \AA$ and $52.3 \AA$. The match of the form factor for the $-2^{\circ}$ only slightly improves the difference at $Q=0.07 \AA^{-1}$. Despite the improved $R$-factor match, the remaining difference in shape points to the necessity of further variations.

\section{Twist About Dyad Axis}

Figure 38 illustrates incrementally twisting $\mathrm{NCP}_{2}$ and $\mathrm{NCP}_{4}$ simultaneously between $\Delta \rho=-45^{\circ}, \ldots, 45^{\circ}$. The resulting $R$ values from comparing these models to experimental data are shown in 39(a). Comparing these structures to the $10 \mathrm{mM} \mathrm{K}^{+}$, we see a tiny improvement by twisting $\mathrm{NCP}_{2}$ and $\mathrm{NCP}_{4}$ by $16^{\circ}$. The $1 \mathrm{mM} \mathrm{Mg}^{2+}$
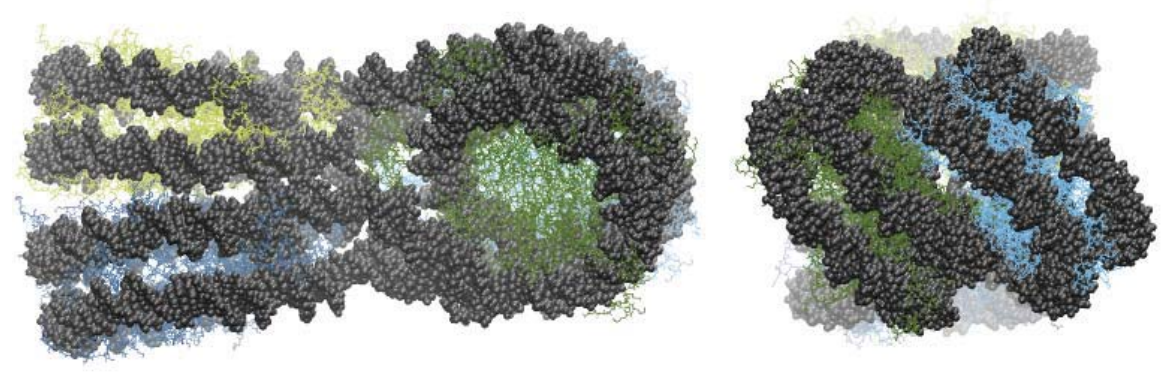

Figure 38. Twist Rigid Body Moves of $\mathbf{N C P}_{2}$ and $\mathbf{N C P}_{4}$ : Adjusting the twist angle of $\mathrm{NCP}_{2}$ and $\mathrm{NCP}_{4}$ by $\Delta \rho=-45^{\circ}, \ldots, 45^{\circ}$. As in fig. 36, the NCP proteins are colored to match the DNA in fig. 35 , namely: $\mathrm{NCP}_{1}-\mathrm{NCP}_{4}$ respectively dark blue, light blue, light green, dark green. (Animated in electronic version, $\Delta \rho=-45^{\circ}$ is shown in the print version.) 

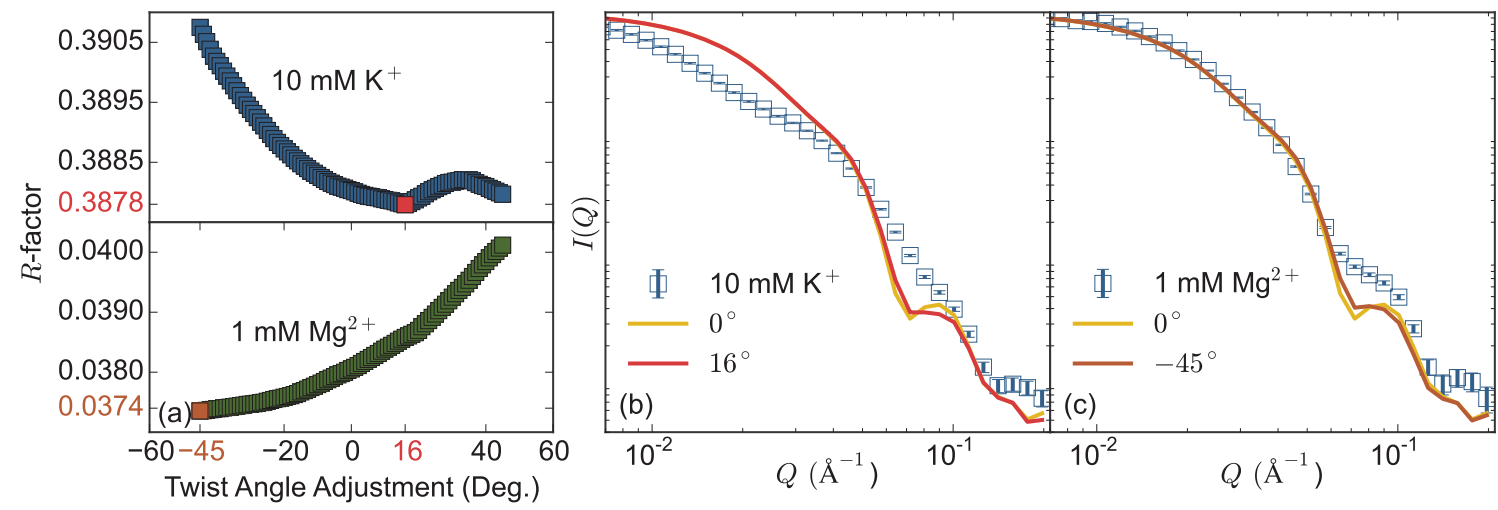

Figure 39. Best-Matched Models from Twist Moves of $\mathbf{N C P}_{2}$ and $\mathbf{N C P}_{4}$ : The $R$-factor comparison (a) and best-matched form factors, (b) and (c), for rotations of $\mathrm{NCP}_{2}$ and $\mathrm{NCP}_{4}$ by $\Delta \rho=-45^{\circ}, \ldots, 45^{\circ}$ about their dyad axes.

does not have an absolute minimum though $\Delta \rho=-45^{\circ}$ rotation reduced the $R$ by $1.8 \%$ to 0.0374 . This indicates a possibility of variation in the twist angle from the 1ZBB configuration but the small improvement in the form factor shape, fig. 39(c), coupled with such a small change in the $R$-factor provides only weak evidence for this variation.

\section{Sliding Stacked NCPs}

We also explored face-on-face sliding of stacked NCPs by rotating them about the axis passing through the tetranucleosome center parallel to their stack axes. These sliding rotations can be compared to how two hands of a clock pass each other, where the center of the clock is the tetranucleosome center. For these rotations, we chose to simultaneously move NCPs as a pair in an asymmetric manner.

A key aspect of the 1ZBB structure is a crystallographic two-fold symmetry axis bisecting the linker DNA between the $\mathrm{NCP}_{2}$ and $\mathrm{NCP}_{3}$. We chose to rotate stacked NCPs by the same angle in opposite directions, without applying the same angle to $\mathrm{NCP}_{2}$ and $\mathrm{NCP}_{3}$, nor $\mathrm{NCP}_{1}$ and $\mathrm{NCP}_{4}$. This independent treatment of stacked NCPs, also used for the twist moves, inherently breaks the 1ZBB crystallographic two-fold symmetry, and we therefore refer to it as the asymmetric method. These moves are 

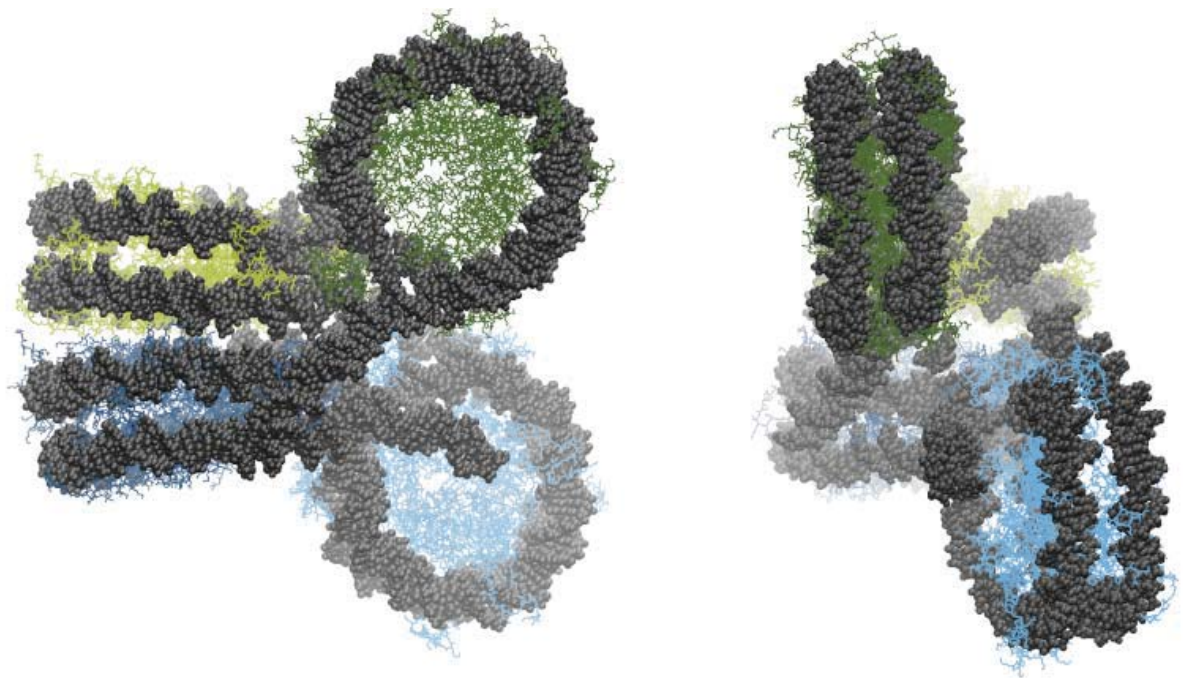

Figure 40. Slide Rigid Body Moves of $\mathbf{N C P}_{2}$ and $\mathbf{N C P}_{4}$ : Sliding stacked $\mathrm{NCP}_{2}$ and $\mathrm{NCP}_{4}$ by rotating them $\Delta \varphi=-60^{\circ}, \ldots, 60^{\circ}$, about the axis passing through the tetranucleosome array center and parallel to their stack axis. As in figs. 36 and 38, the NCP proteins are colored to match the DNA in fig. 35 , namely: $\mathrm{NCP}_{1}-\mathrm{NCP}_{4}$ respectively dark blue, light blue, light green, dark green. (Animated in electronic version, $\Delta \varphi=-60^{\circ}$ is shown in the print version.)

illustrated in fig. 40 showing rotation of the $\mathrm{NCP}_{2}$ and $\mathrm{NCP}_{4}$, and fig. 42 showing the rotation of all 4 NCPs. We define a positive rotation angle of $\mathrm{NCP}_{i}$ as increase its center-to-center distance from $\mathrm{NCP}_{i-1}$ and decrease its center-to-center distance to $\mathrm{NCP}_{i+1}$, e.g., $\mathrm{NCP}_{2}$ further from $\mathrm{NCP}_{1}$ and closer to $\mathrm{NCP}_{3}$. When rotating stacked NCPs by the same angle, this directional definition inherently rotates them in opposite directions when referencing their shared stack axis.

Figure 41 shows the $R$ values and form factor comparisons for asymmetric rotation of $\mathrm{NCP}_{2}$ and $\mathrm{NCP}_{4}$ by $\Delta \varphi=-60^{\circ}, \ldots, 60^{\circ}$. Compared to the $10 \mathrm{mM} \mathrm{K}^{+}$data, the best-matched model, $R=3.6$, rotates both NCP by $\Delta \varphi=-41^{\circ}$, for a combined angle of $82^{\circ}$ between them, thereby approximating an extended structure. This adjustment improves the match primarily between $0.02 \AA^{-1}<Q<0.09 \AA^{-1}$ with the shape of the form factors, fig. 41(c), still differing significantly at low $Q$-values. For the $1 \mathrm{mM}$ $\mathrm{Mg}^{2+}$ data, none of the rotated models produced an improved $R$-factor. We note that the structural symmetries between positive and negative rotations produce the 

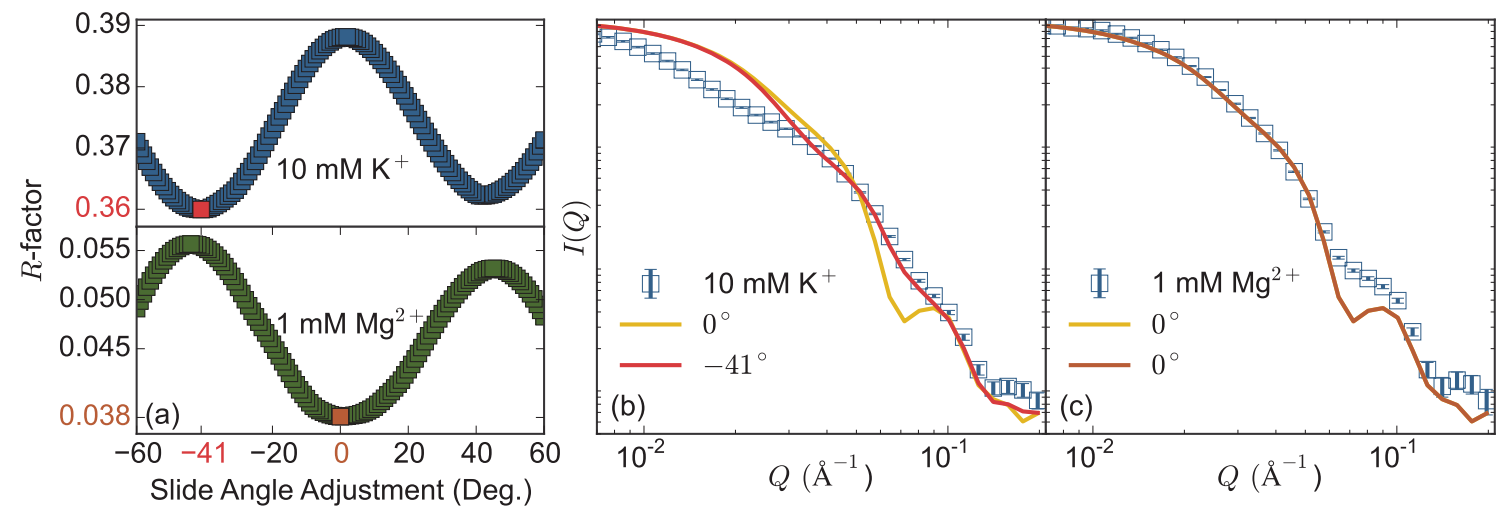

Figure 41. Best-Matched Models from Slide Moves of $\mathbf{N C P}_{2}$ and $\mathbf{N C P}_{4}$ : The $R$-factor comparison, (a), and best-matched form factors, (b) and (c), for sliding stacked $\mathrm{NCP}_{2}$ and $\mathrm{NCP}_{4}$ using an angle $\Delta \varphi=-60^{\circ}, \ldots, 60^{\circ}$.

similar scattering profiles as demonstrated by the symmetric nature of fig. 41(a).

To further investigate the possibility of NCP sliding, we coupled the rotation angle of all four NCPs incrementing the value between $\Delta \varphi=-60^{\circ}, \ldots, 60^{\circ}$, animated in fig. 42. The $R$-factor for both experimental cases produce nearly symmetric results about the starting configuration, shown in fig. 43. This results from rotational sym-
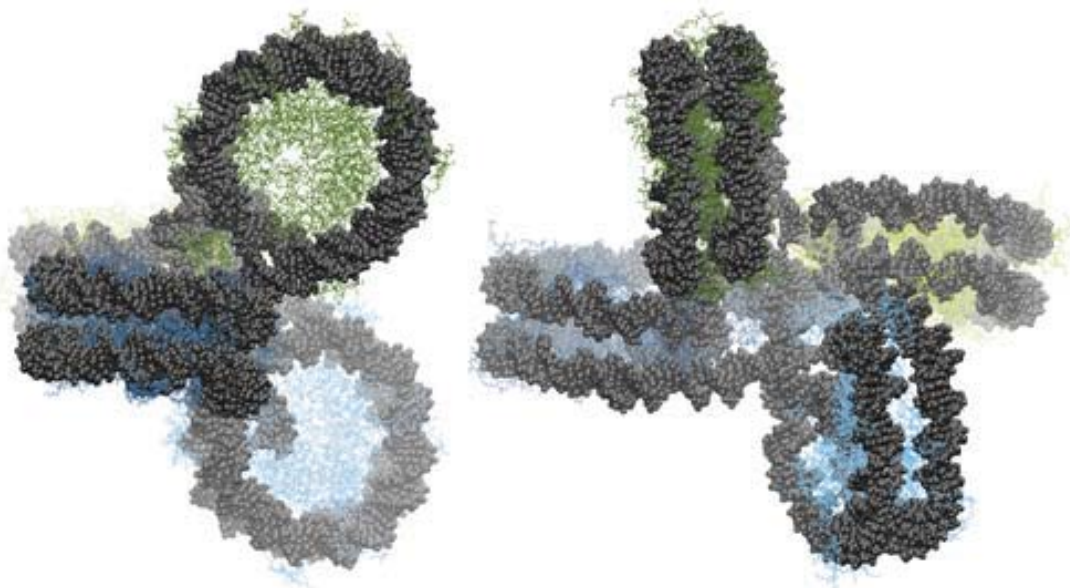

Figure 42. Slide Moves of All NCPs: Sliding both pairs of stacked NCPs by rotating them $\Delta \varphi=-60^{\circ}, \ldots, 60^{\circ}$ about the axes passing through the nucleosome center parallel to their respective stack axes. As in figs. 36, 38 and 40, the NCP proteins are colored to match the DNA in fig. 35, namely: $\mathrm{NCP}_{1}-\mathrm{NCP}_{4}$ respectively dark blue, light blue, light green, dark green. (Animated in the electronic version, $\Delta \varphi=-60^{\circ}$ is shown in the print version.) 

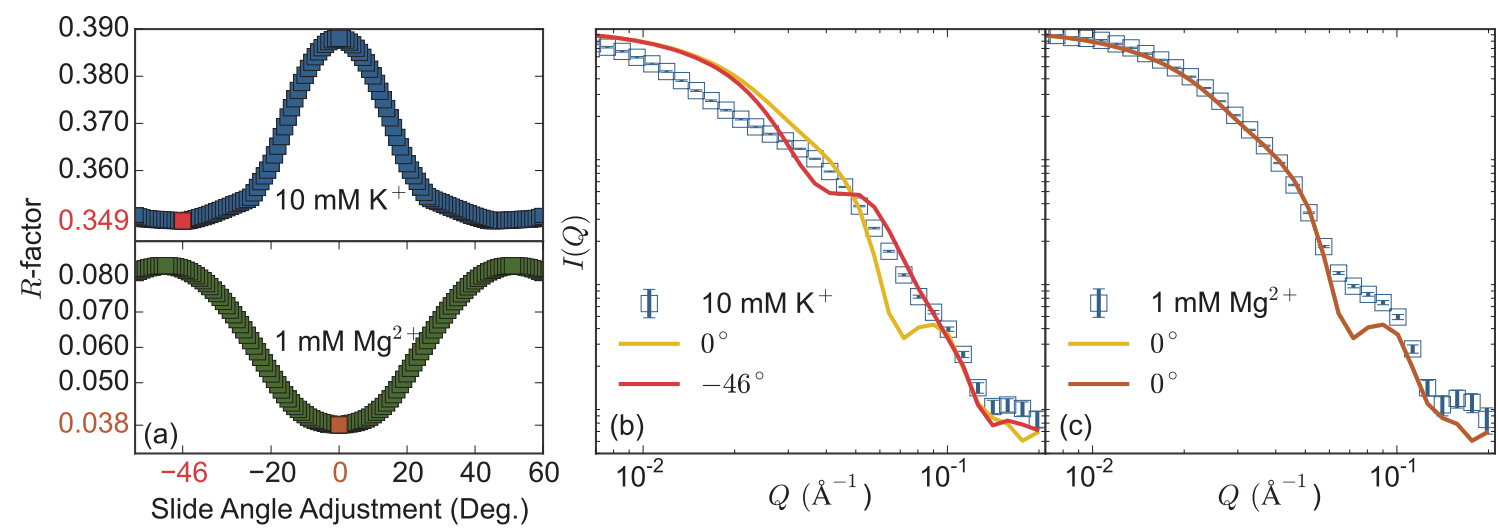

Figure 43. Best-Matched Models from Slide Moves of All NCPs: The $R$ factor comparison, (a), and best-matched form factors, (b) and (c), for sliding both pairs of stacked NCPs.

metry which transforms between models with negative and positive rotation angles. For $1 \mathrm{mM} \mathrm{Mg}^{2+}$ all adjustments increase the $R$, similar to only rotating $\mathrm{NCP}_{2}$ and $\mathrm{NCP}_{4}$.

To more fully explore asymmetric sliding rotations, we separately scanned $\Delta \varphi_{13}$ and $\Delta \varphi_{24}$ incrementally between $-60^{\circ}, \ldots, 60^{\circ}$. The comparison to $10 \mathrm{mM} \mathrm{K}^{+}$and $1 \mathrm{mM} \mathrm{Mg}^{2+}$ are shown in fig. 44 panels (i) and (ii) respectively. The results from

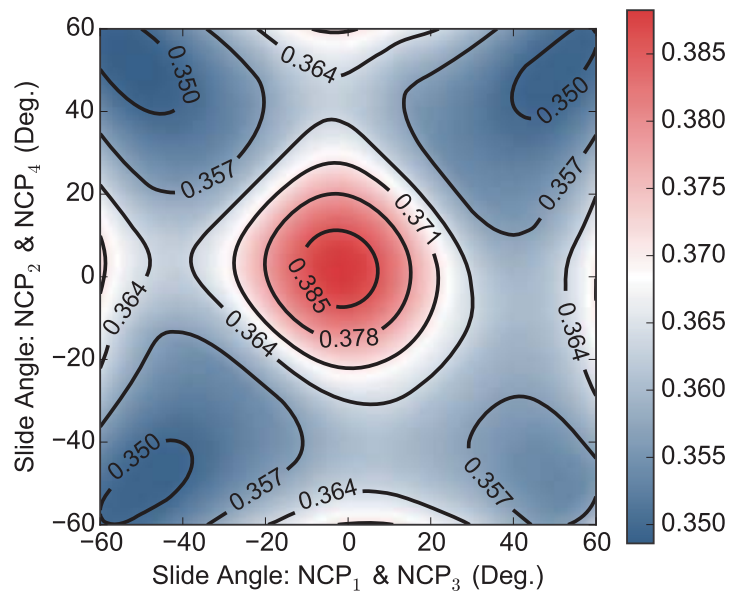

(i) $10 \mathrm{mM} \mathrm{K}^{+}$

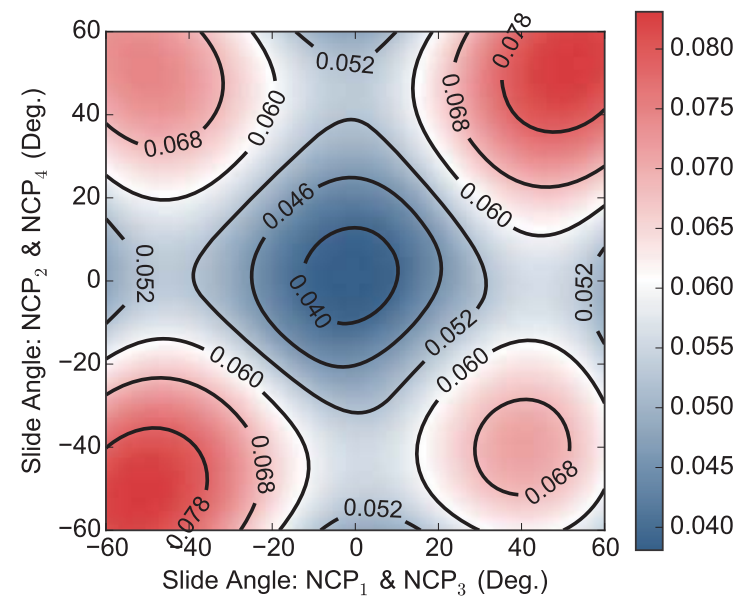

(ii) $1 \mathrm{mM} \mathrm{Mg} \mathrm{Mg}^{2+}$

Figure 44. Scan of Rotation Angles: The $R$-factor calculation for every structure generated using a scan of rotation angles between $-60^{\circ}, \ldots, 60^{\circ}$. For these scans, we coupled the rotations of $\mathrm{NCP}_{1}$ with $\mathrm{NCP}_{3}$ and $\mathrm{NCP}_{2}$ with $\mathrm{NCP}_{4}$, rotating the coupled NCPs by the same angle. 
figures 41(a) and 43(a) are respectively contained in these contour plots along the vertical center line and the diagonal line from the lower left to the upper right corner.

Of the 14641 structures generated, the 842 and 408 best structures are respectively contained in the region labeled as $R=0.350$ for the $10 \mathrm{mM} \mathrm{K}^{+}$and $R=0.040$ for the $1 \mathrm{mM} \mathrm{Mg}^{2+}$. Compared to the starting structure, the $R$-factor limit for the $10 \mathrm{mM}$ $\mathrm{K}^{+}$sub-ensemble represents an improvement of $\sim 10 \%$ while the $R$-factor limit for the $1 \mathrm{mM} \mathrm{Mg}^{2+}$ sub-ensemble shows no improvement.

\section{Discussion}

We see from these user-directed rigid body modeling efforts that using the simple transformations discussed, we are able to produce models which only agree slightly better with the experimental results compared to the 1ZBB crystal structure. Improvements were most pronounced for the $4 \times 167 \mathrm{gH} 5$ array in $10 \mathrm{mM} \mathrm{K}{ }^{+}$which differed more from the starting structure. For the $1 \mathrm{mM} \mathrm{Mg}^{2+}$ data, the difference at

$0.07 \AA^{-1}$ persisted for all structures that matched the $R_{g}$ of the experimental data. Though some models did not show this dip at $0.07 \AA^{-1}$, the $R$-factor increased for these structures because the low- $Q$ region varied from the experimental value. This low- $Q$ region encodes the $R_{g}$ of the model, one of the most fundamental qualities of a structure. In order to find a means of performing rigid body moves which could sample the types of rigid body moves attempted, but in a way that could maintain the $R_{g}$, we developed a DNA MC simulation algorithm discussed in Chapter 4 .

\section{Chapter Summary}

In this chapter, we presented the sample preparation and experimental measurements of nucleosome arrays with $2,3,4$, and 12 NCPs. We have provided details on the protein and DNA expression and purification, as well as the octamer and nucleosome array reconstitution. We discussed the SAXS experiments, detailing the measurement 
and data reduction processes. We outlined the zero concentration extrapolation to obtain the form factor when data acquired at different concentrations show minor interparticle interactions and it is not feasible to measure at lower concentrations. We also reported the user-directed rigid body modeling of $4 \times 167$ gH5 arrays.

From the array form factors, we identified several key observations. Independent of the number of nucleosomes, the form factors show a consistent salt-dependent change most visible in the decrease of the $Q=0.040 \AA^{-1}$ Kratky peak as ion concentration increases. The addition of gH5 linker histone to $4 \times 167$ arrays produces minimal change in low salt but a significantly modified structure in higher salt. The agreement between the calculated form factor from the 1ZBB model and experimental form factor from the $4 \times 167 \mathrm{gH} 5$ array in $1 \mathrm{mM} \mathrm{Mg}^{2+}$ indicate a solution structure unquestionably similar to the crystal structure.

Using geometric transformations to modify the 1ZBB structure, we demonstrate models with small improvements when compared with experimental results of the $4 \times 167$ gH5 array in $10 \mathrm{mM} \mathrm{K}^{+}$and $1 \mathrm{mM} \mathrm{Mg}^{2+}$. The most significant change for the $1 \mathrm{mM} \mathrm{Mg}^{2+}$ data results from twisting two stacked nucleosomes about their dyad axes though minimal improvements are shown from decreasing the opening angle, or stack distance, while no improvements were achieved by sliding stacked nucleosomes. For $10 \mathrm{mM} \mathrm{K}^{+}$, though the agreement is not exemplary, the best-matched models indicate a large separation of stacked nucleosomes, either through increasing the opening angle or sliding stacked NCPs apart, both of which approximate an extended structure.

Though we could further explore combinations of geometric transformations in an effort to obtain models with better agreement, e.g., adding other moves to maintain the $R_{g}$ while adjusting NCP positions, the number of different combinations and perturbations would be unmanageable. Additionally, the opening, twist and sliderotation transformations discussed are only a tiny subset of those possible. While these transformations seem reasonable, the total number of degrees of freedom for 
modeling nucleosomes far surpasses that of manual geometric modifications. Considering each linker DNA has over a thousand atoms, each bonded to 1-4 other atoms and interacting with many more, the complexity of the system calls for a more automated approach. Additionally, these directed rigid body moves made essentially no progress in resolving the small discrepancy between the 1ZBB model and the data from $4 \times 167 \mathrm{gH} 5$ arrays in $1 \mathrm{mM} \mathrm{Mg}^{2+}$. We generated improved models for describing the $4 \times 167 \mathrm{gH} 5$ arrays in $10 \mathrm{mM} \mathrm{K}^{+}$, but even the scattering profiles from the bestmatched structures showed significant differences. With the goal to achieve models which could rapidly explore a much larger amount of configuration space, we generated a CG MC simulation algorithm to sample DNA torsions thereby generating an ensemble of atomic models, discussed in Chapter 4. 


\title{
Chapter 4: Double-Stranded DNA Simulation Algorithm
}

\section{Chapter Overview}

Motivated to produce ensembles of atomic models in agreement with the experimental results described in Chapter 3, we created an algorithm for generating modified dsDNA structures. The SASSIE [109] program provides a similar algorithm for generating ensembles of modified protein, carbohydrate, and single-stranded nucleic acid (ssNA) structures. We designed our algorithm using the SASSIE application programming interface (API) to take advantage of the existing framework for managing molecular structures. Adopting this API allowed us to fully integrate our dsDNA module into the SASSIE program to facilitate simultaneous modeling of protein, ssNA, and dsDNA, e.g., simulating MC moves of both the linker DNA and flexible histone tails of nucleosome arrays.

\section{Coarse-Grain Monte Carlo Simulation Algorithm for Double- Stranded DNA}

\author{
Steven C. Howell, ${ }^{\dagger}$ Xiangyun Qiu, ${ }^{\dagger}$ and Joseph E. Curtis, ${ }^{\ddagger}$ \\ †Department of Physics, George Washington University, Washington, DC; \\ $\ddagger$ NIST Center for Neutron Research, National Institute of Standards and Technology, \\ Gaithersburg, Maryland \\ In preparation
}

\begin{abstract}
The growing interest in determining structures of macromolecules containing DNA in solution and their structure-function relationship has encouraged experiments using methods such as solution SAXS and SANS. One such DNA system of interest is the DNA-protein complex known as the nucleosome, the building block of chro-
\end{abstract}


matin in eukaryotic cells. Solution SAXS and SANS provide a means to explore these macromolecules in a cellular-like environment, but there exists a need for open source software to generate atomic structures of DNA that subsequently allow for the comparison to experimental results. We present an algorithm which uses an all-atom model for a biological macromolecule containing DNA to rapidly generate an ensemble of atomic models, which can then be compared with experimental data. This algorithm represents user designated flexible DNA using a CG wormlike chain model, samples dsDNA bend and twist dynamics, then recovers an all-atom model based on the final CG configuration, for which the strains created between CG beads can be relaxed using energy minimization. Using this algorithm on commodity computing hardware, one can rapidly generate an ensemble of molecular models, with atomic details not otherwise available. Together with SAXS or SANS data, such an ensemble can be used to provide clear insight into experimental scattering results thereby elucidating the solution structure of DNA macromolecules. We demonstrate the ensemble results from simulations applying this algorithm to a single nucleosome and an array of four nucleosomes. Compared to currently available software resources, the modeling capability provided by this algorithm will revolutionize experiment driven modeling of macromolecules containing DNA, particularly nucleosomes and nucleosome arrays. The MC DNA algorithm has been implemented on a web user interface and is available for general use (https://sassie-web.chem.utk.edu/sassie2/).

\section{Introduction}

For many macromolecules, including nucleosomes, a wealth of structure knowledge has been obtained using experimental techniques which examine the macromolecule in a static environment; such techniques include X-ray crystallography, atomic force microscopy, and cryo-electron microscopy.

The conformation and dynamics of DNA directly control gene directed processes 
in biological systems at the fundamental level. By studying dsDNA in a static environment, precise structures have been reported for DNA as a double helix [112, 113], DNA packaged in a nucleosome [44], and DNA packaged in a tetranucleosome array [2]. Precise results in solution are confined to the complexity of single nucleosome and limited in their scope [28, 29, 32].

The methods of SAXS and SANS are well suited for studying dynamic DNA structures in a solution environment. In SAS, a beam of collimated radiation (Xrays or neutrons) incident on a sample interacts with inhomogeneities in the sample matter. The elastic scattering from these interactions encodes the temporal and spatial average of the distance distribution between atomic structures on the length scales of $10-5000 \AA$. This ensemble average provides structure information such as the overall size and shape of the scattering particles, e.g., molecular weight and radius of gyration, as well as characteristic distances between units or subunits in the system.

More detailed insight regarding the solution structures of molecules can be gained from experimental scattering by comparing the results to the theoretical scattering intensity of model structures. Various techniques are currently available for creating and modifying model structures; these techniques employ methods such as rigid body modeling [114, 115], 3D mesh generation [116], CG simulations [117-123], ab initio modeling using dummy spheres [69, 124], and user-directed transformation of all-atom molecular structures $[28,29,125]$. These methods lack the ability to rapidly modify pre-existing all-atom models to generate an ensemble of all-atom models.

For many studies of biological molecules, a great deal of information is known about the molecular structure, or the structure of its subunits, prior to performing SAS experiments. For example, when it is possible to crystallize a molecule, X-ray crystallography can be used to obtain an electron density map for the structure in this crystallized environment. With sufficient resolution, such a density map can be converted to an all-atom structure model from which the theoretical scattering 
for the individual molecule can be calculated. Comparing the experimental solution scattering profile to the scattering calculated from this crystallized structure model can identify qualitative differences between the structure of the molecule in solution compared to when it is crystallized. Additionally, one could then modify the all-atom model of the crystal structure to generate potential model structures for which the calculated scattering would better match the experimental results. For molecules composed of proteins, carbohydrates, and ssNA, SASSIE [109] provides this exact functionality, to rapidly modify an all-atom structure model to generate an ensemble of configurations which can be compared to experimental data. No such algorithm exists for modifying an atomic model of dsDNA to generate varied configurations in a timely manner.

In this report, we present an application of a CG MC move algorithm, applied to all-atom B-form dsDNA, which rapidly generates an ensemble of all-atom macromolecular structures. Figures 45 and 46 respectively illustrate the CG model and a schematic of the algorithm. We validate the implementation of the model energetics by comparing simulation results to the accepted properties of B-form DNA. We then demonstrate that a short energy minimization of resulting structures relaxes the discontinuities caused by the CG dynamics enabling this algorithm to generate robust atomic models. Finally, we provide example ensembles of structure models for a single nucleosome and a short array of nucleosomes filtered against sample SAXS profiles. This method of filtering an ensemble using experimental SAS data provides a powerful means for representing the structure of DNA macromolecules in solution. Software implementing this algorithm is available for public use at, https://sassie.web.chem.utk.edu/sassie2/. Additionally, the code is openly available to encourage external development and contributions.

We emphasize the significant benefit this algorithm brings to modeling SAS experiments of nucleosomes and nucleosome arrays. In nucleosomes, the DNA-protein 


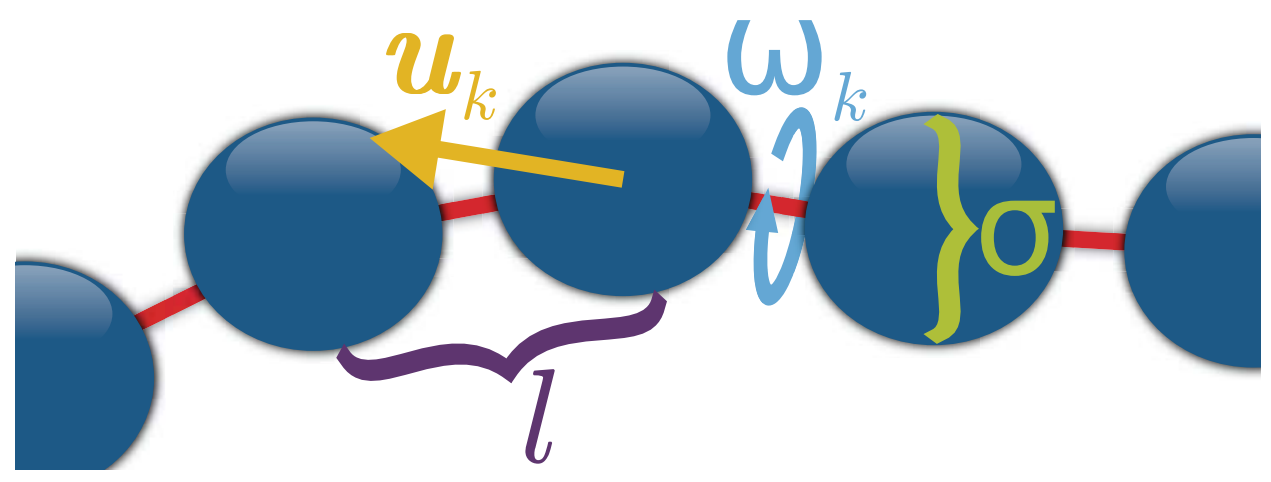

Figure 45. Illustration of the Bead-Rod Model: The CG beads each have width $\sigma$ and are connected by a inextensible rod of length $l$. The unit vector $\boldsymbol{u}_{k}$ describes the orientation of the $k^{\text {th }}$ rod. The angle $\omega_{k}$ represents the twist angle between beads $k$ and $k+1$. This model build on the bead-rod model presented by Wang et al. [126] through the addition of the twist dynamic.

hybrid that forms the building block for chromatin, DNA bending, twisting, and unwrapping are the dominant mechanisms for structural modifications of the macromolecular structure. The most detailed structural information for nucleosomes has been obtained using crystallography [2, 44], or cryo-electron microscopy [27], methods which explore the static structure of nucleosomes in a biologically artificial environment. SAS has been used to study nucleosomes and nucleosome arrays in solution but efforts to find model structures that match experimental observations have been limited to techniques such as manually modifying structures [29] and substituting linear DNA in place of protein-bound DNA [28]. The ability to rapidly vary the conformation of DNA will provide drastically improved means to model structural biology problems where dsDNA plays a role in general, but especially for modeling solution scattering data of nucleosomes and nucleosome arrays.

\section{Method}

The number of atoms in typical DNA molecules studied using SAS [28-30, 32, 127, 128] makes a MD approach intractable using commodity hardware. Even high performance computing simulations may not sample enough structural configurations 
to find structures that match experimental results, or at least not in a reasonable amount of time. To overcome this limitation, starting from an all-atom model, we replace each flexible DNA bp with a CG bead, sample a bend or torsion angle between the CG DNA, then reinsert the all-atom DNA coordinates in relation to the modified CG coordinates. We eliminate bond strain resulting at the joint between DNA beads by energy minimizing each of the final all-atom structures. Wall clock estimates using a standard desktop computer indicate that, without minimization, the algorithm would require 24 hours to generate 430000 different structures for a 3800 atom 60 bp linear DNA model, and 700 different structures for a 107000 atom tetranucleosome DNA-protein model. For these estimates, we designated the middle 58 bp of the linear DNA as flexible, and we designated five separate $20 \mathrm{bp}$ DNA segments as flexible in the tetranucleosome model.

The CG representation we use to represent B-form DNA is based on the bead-rod model presented by Wang et al. [126], which originates from the classical wormlike chain model [129]. This simple model, illustrated in fig. 45, represents DNA using $N$ beads connected by $N-1$ inextensible rods of length $l$, for a total contour length, $L=(N-1) l$. The energetics of this model employs a bending penalty between adjacent beads and an excluded volume repulsion between beads. The bending energy, $U_{\text {bend }}$, is obtained by discretizing the wormlike chain model,

$$
U_{\text {bend }}=\frac{k_{B} T}{2} L_{p} \int_{0}^{L}\left(\frac{\partial \boldsymbol{u}}{\partial s}\right)^{2} d s \quad \rightarrow \quad U_{\text {bend }} \approx k_{B} T \frac{L_{p}}{l} \sum_{k=1}^{N-2}\left(1-\boldsymbol{u}_{k} \cdot \boldsymbol{u}_{k+1}\right)
$$

where $k_{B}$ is the Boltzmann constant, $T$ is the temperature, $L_{p}$ is the persistence length of DNA $\left(534 \AA\right.$ in $\left.10 \mathrm{mM} \mathrm{Na}^{+}[130,131]\right)$, and $\boldsymbol{u}_{k}$ is the unit vector along the $k^{\text {th }}$ rod. This bending energy term accounts for non-bonding electrostatic interactions of the DNA molecule using a salt dependent persistence length. An additional energy term representing the excluded volume between any two beads, $i$ and $j$, separated 
by a distance $r_{i j}$, is represented using a Weeks-Chandler-Andersen (WCA) form of a purely repulsive Lennard-Jones potential,

$$
U_{\mathrm{WCA}}=\left\{\begin{array}{lc}
4 k_{B} T\left[\left(\sigma / r_{i j}\right)^{12}-\left(\sigma / r_{i j}\right)^{6}+1 / 4\right] & r_{i j}<2^{1 / 6} \sigma \\
0 & r_{i j} \geq 2^{1 / 6} \sigma
\end{array},\right.
$$

where the chain width, $\sigma$, determines the range of the interaction.

Expanding on this simple bead-rod model, we added a harmonic twist energy to account for twisting between subsequent bps,

$$
U_{\text {twist }}=\frac{k_{B} T}{2} \kappa \sum_{k=1}^{N-1}\left(\omega_{k}-\bar{\omega}\right)^{2},
$$

where the force constant, $\kappa=0.062 \times\left(1^{\circ}\right)^{-2}$, is the average Twist-Twist force constant, $\omega_{k}$ is the twist-angle between bps $k$ and $k+1$, and $\bar{\omega}=+35.4^{\circ}$ is the average twist angle between bps [132]. Sampling twist dynamics based on this established harmonic twist energy allows for increase conformational sampling while maintaining the appropriate major and minor groove widths in the simulated DNA.

Though this bead-rod model and its typical level of granularity are best suited for long DNA $\left(L>L_{p}\right)$, we have found it effective in rapidly modifying all-atom models of short DNA structures $\left(L<L_{p}\right)$ by adding a HS potential between atoms after the reverse CG process. A typical DNA system studied using SAS contains 10-100 bp of DNA for which small conformational changes significantly change the solution scattering $[28,29,32,128]$, much smaller than the DNA length this bead-rod model was originally designed to represent.

With such short DNA, and to maximize the range of structures generated, we represent flexible DNA regions using a single CG bead for each bp. Additionally, this simplifies the implementation of the twist energetics as using several bps for each bead would concentrate the twist dynamics of all those bps into the single bond connecting 
that bead to the adjacent beads. One bp per bead translates to an average rod length between beads of $l=3.38 \AA$ and a chain width of $\sigma=3.01 \AA$. As $3.01 \AA$ is much smaller than the width of a DNA chain, the WCA energy term does not fully enclose all the atoms it represents and is therefore not sufficient to prevent overlap between atoms in the final all-atom structures. We avoid such overlap using an additional steric HS potential at both the CG and all-atom levels. At the CG level, we use a HS diameter of $19 \AA$, corresponding to the atomic width B-form DNA, but only apply this restriction to beads separated by at least 6 beads, or roughly $20 \AA$ in either direction along the DNA chain. After reinserting the transformed all-atom group back into the complete molecule, we use a HS diameter of $0.8 \AA$.

To generate an ensemble of all-atom structures using this DNA model, the algorithm iterates over a multi-step process. Figure 46 illustrates this process for a $40 \mathrm{bp}$ linear DNA fragment with $10 \mathrm{bp}$ of flexible DNA. The algorithm randomly selects one of the user designated flexible groups, replaces each flexible DNA bp with a CG bead positioned at the bp reference frame origin [110], then stores the coordinates of the bp atoms with respect to that reference frame. The algorithm then generates a new structure for the group by selecting one of the CG DNA beads and samples a torsion about one of the three coordinate axes of the DNA bp reference frame. All successive beads and any other post atoms are transformed with respect to the sampled torsion. This new group structure is accepted according to Maxwell-Boltzmann statistics using the energies in eqs. (30)-(32) and under the requirement that no bead nor atoms overlap. After a new group structure is accepted, the algorithm recovers an all-atom representation for the flexible DNA by reinserting the atoms for each CG bead based according to the new origin and orientation. This all-atom group structure is then reinserted into the complete atomic molecule after verifying that the coordinates of the altered group atoms do not overlap with the other atoms in the molecule. This selection, CG, sampling, and overlap check cycle is repeated for the designated num- 


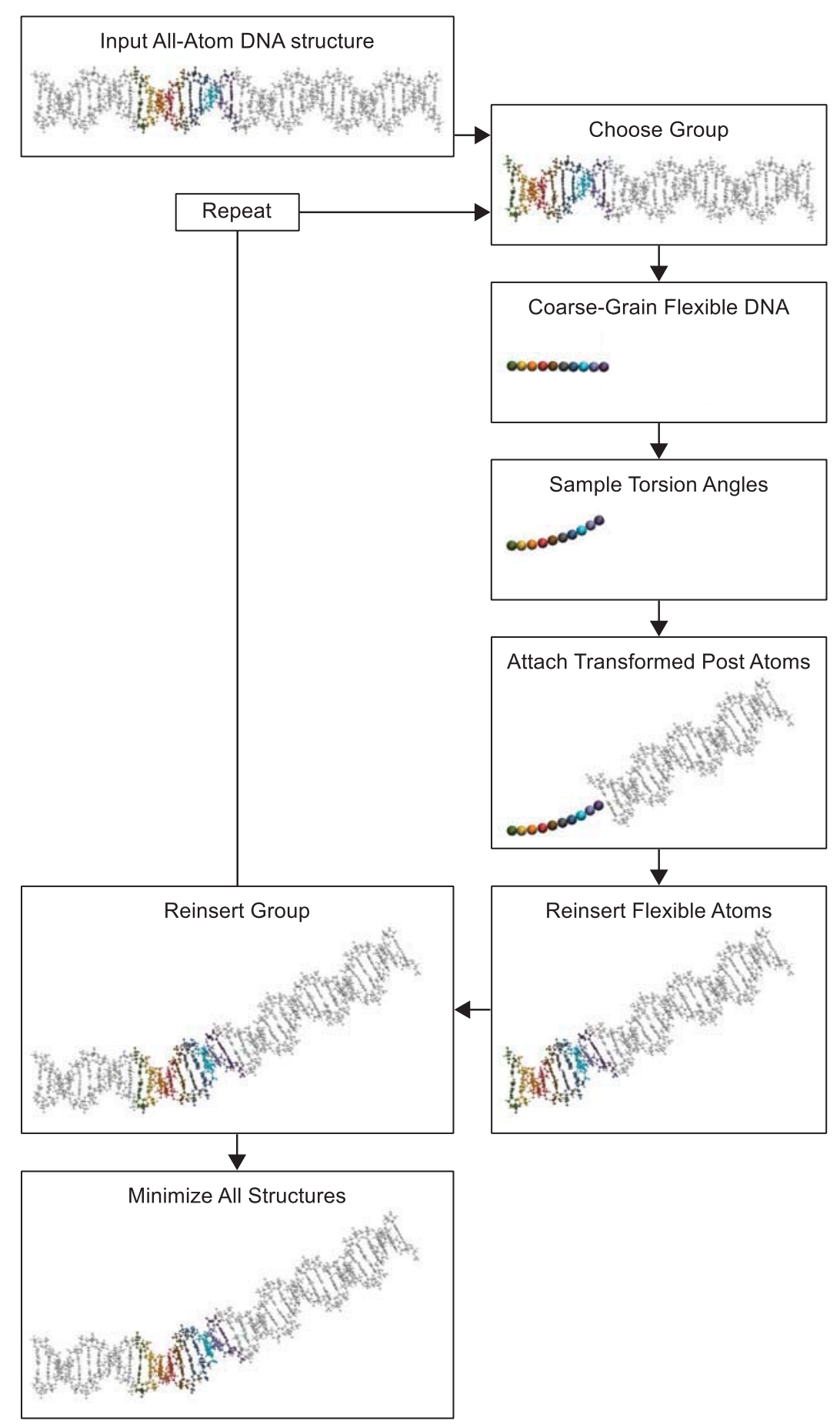

Figure 46. Schematic of the DNA Monte Carlo Algorithm: Illustration of MC sampling for a $40 \mathrm{bp}$ linear DNA segment with $10 \mathrm{bp}$ of flexible DNA. Each flexible bp is uniquely colored. Note that the chain width used in the WCA energy, $\sigma=3.01 \AA$, does not enclose the volume of the atoms represented by each bead. Consequently, the resulting energies do not fully prevent overlap of atoms after reinserting all-atoms back on the transformed beads. We prevent such overlap by including a HS potential between beads separated by more than $6 \mathrm{bp}$. This HS diameter is set at $19 \AA$, the atomic width of B-form DNA. 
ber of trial steps. As a final step, all structures must be energy minimized to relax atomic bond strains between flexible DNA bps.

A key part of this multi-step process is the mechanism of representing a DNA bp using a single bead, then reinserting the atoms from that bp back onto the bead after performing MC dynamics. The first step in this process is to determine the coordinates of the atoms within each bp with respect to the standard DNA bp reference frame, defined by Olson et al. as follows [110]. In this reference frame, the $x$-axis is the perpendicular bisector of the $\mathrm{C} 1^{\prime} \ldots \mathrm{C} 1^{\prime}$ vector spanning the bp and points in the direction of the pseudo-dyad axis of an ideal Watson-Crick bp. The $y$-axis runs parallel to the $\mathrm{C} 1^{\prime} \ldots \mathrm{C} 1^{\prime}$ vector, points in the direction of the sequence strand, and passes through the intersection of the $x$-axis and the vector connecting the pyrimidine $\mathrm{Y}(\mathrm{C} 6)$ and the purine $\mathrm{R}(\mathrm{C} 8)$ atoms. The $z$-axis is defined as the cross product of the $x$ and $y$ axes, i.e., $\boldsymbol{z}=\boldsymbol{x} \times \boldsymbol{y}$, and consequently points along the $5^{\prime}$ to $3^{\prime}$ direction of the sequence strand. Once this reference frame has been determined, A CG bead is placed at the bp center. In addition to tracking the position of each bead through the MC dynamics, the orientations of each bp axis are updated for each accepted move. This allows the reinsertion of the bp coordinates after performing any number of $\mathrm{MC}$ steps. An unavoidable consequence of performing $\mathrm{CG}$ dynamics is that atomic bonds between DNA bps becomes compressed or extended in response to the bp torsions. A short energy minimization relaxes these strains. This CG then reverse-CG process sufficiently reduces the complexity of performing MC dynamics thereby facilitating the rapid generation of atomic models by varying the DNA structure in an energetically sampled manner.

Compared to A-form and Z-form DNA, B-form DNA is uniquely suited to this bead-rod representation of the wormlike chain using one bp per bead as the DNA bp centers lie approximately on the line passing through the center of the DNA, visible in the "Coarse-Grain Flexible DNA" step of fig. 46. This requirement, that 
the bead centers align with the center of the represented atomic chain, is imposed by the bending energetics, described in eq. (30). Consequently, the current energetics contained in this model are not sufficient to simulate A-form and Z-form DNA.

To evaluate how well the generated model structures represent experimental SAS data, one must calculate the theoretical scattering intensity from each structure in the final ensemble. For model structures, the scattering intensity vs momentum transfer $(I(Q)$ versus $Q)$ is calculated, in the simplified case of uniform scattering power of all atoms, using:

$$
I(Q)=4 \pi \int_{0}^{\infty} P(r) \frac{\sin (Q r)}{Q r} d r,
$$

where $Q=4 \pi \sin (\vartheta) / \lambda, \lambda$ is the incident (photon or neutron) wavelength, $2 \vartheta$ is the scattering angle, and $P(r)$ is the pair distance distribution function describing the probability of atoms being separated by a distance $r$. For the examples we show in figs. 52 and 53, we calculated the theoretical scattering intensities using the opensource application FoXS $[108,133]$, which calculates $I(Q)$ in a two step process. This application first determines $P(r)$ by explicitly calculating all the interatomic distances and implicitly modeling the first hydration layer of the molecule. It then performs a numerical integration at each point in $Q$ according to eq. (33).

\section{Validation}

A primary objective of this algorithm is to generate atomic models of physically realizable biological molecules which could then be selectively studied further. As such, it is critically important that the torsion energetics, both bending and twisting, agree with reported experimental results and accepted theory. We validated the energetics of the $\mathrm{MC}$ sampling by comparing the structural properties from simulation results to previous studies. Further, we justified the process of reinserting all-atoms back on transformed CG beads by comparing the dihedral angles of resulting structures to those obtained using all-atom MD simulations. 
To validate the bending energetics of the bead-rod model, we calculated the rootmean-square end-to-end distance, $R_{e}$, and the radius of gyration, $R_{g}$, for DNA ranging in length from $265 \AA$, to $10600 \AA\left(0.5-20 \times L_{p}\right)$ using only the energy terms in eqs. (30) and (31). For these simulations, we used two different sets of parameters for the rod length between beads, $l=51.6 \AA$, in direct comparison to the implementation of Wang et al. [126], and $l=3.38 \AA$, to reproduce our implementation of 1 bead for each DNA bp. For both conditions, the width used in eq. (31) was set using $\sigma=2^{-1 / 6} l$ so that $\sigma$ provides a meaningful representation of the width of the chain. As seen in fig. 47, the simulated calculations under both conditions agree with the accepted wormlike chain theory curves [134] and experimental measurements [135]. Each calculated value represents the average of over 300 configurations sampled every $10^{5}$ steps after an initial equilibration of $10^{6}$ steps.

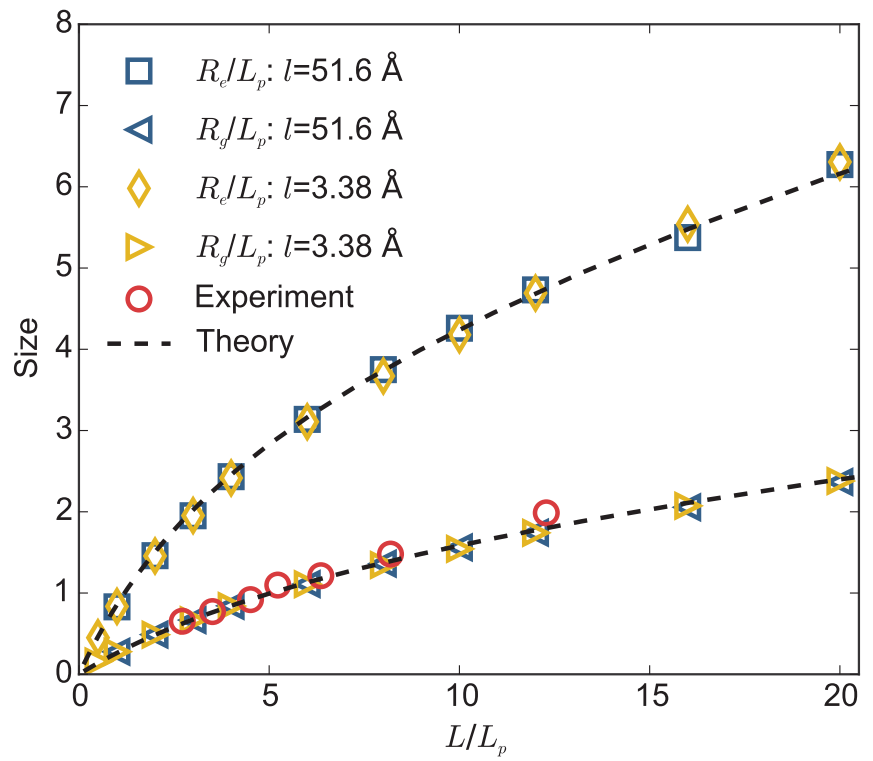

Figure 47. Bulk Properties of the Wormlike Bead-Rod Model: Comparison of the end-to-end distance, $R_{e}$, and the radius of gyration, $R_{g}$, from simulations (blue and yellow symbols) versus the wormlike chain theory [134] (lines) and experimental $R_{g}$ measurements [135] (red circles). The $R_{e}$ and $R_{g}$ values are normalized by the DNA persistence length, $L_{p}$. These MC simulations were based solely on the energy terms in eqs. (30) and (31), with the length between CG beads, $l$, set at either $51.6 \AA$ (blue), to reproduce the results reported by Wang et al. [126], or $3.38 \AA$ (yellow) to correspond to one bp per CG bead. Error values are smaller than the marker size. 
To validate our implementation of the twist energetics, we performed a MC simulation sampling only twist torsions, described by eq. (32). Figure 48 shows the twist angles between 58 flexible DNA bps over 350000 MC steps. Following an initial equilibration of 1000 steps, we calculated the twist angle between flexible bps every $100 \mathrm{MC}$ steps (gray dots). The solid dark blue and dashed yellow lines respectively illustrate the variance in our MC result with those from the crystal structure survey of DNA-protein complexes [132] by plotting the angles that are two standard deviations, $2 \sigma_{\mathrm{SD}}$, from the mean. The mean twist angle in the MC generated structures, light blue, closely matches the expected $+35.4^{\circ}$ determined from crystal structures and used as the equilibrium, $\bar{\omega}$, in the harmonic energetics. The smaller standard deviation seen for $\mathrm{MC}$ generated structures indicates that $\kappa=0.062 \times\left(1^{\circ}\right)^{-2}$ is a conservative selection, well within the limits for B-form DNA.

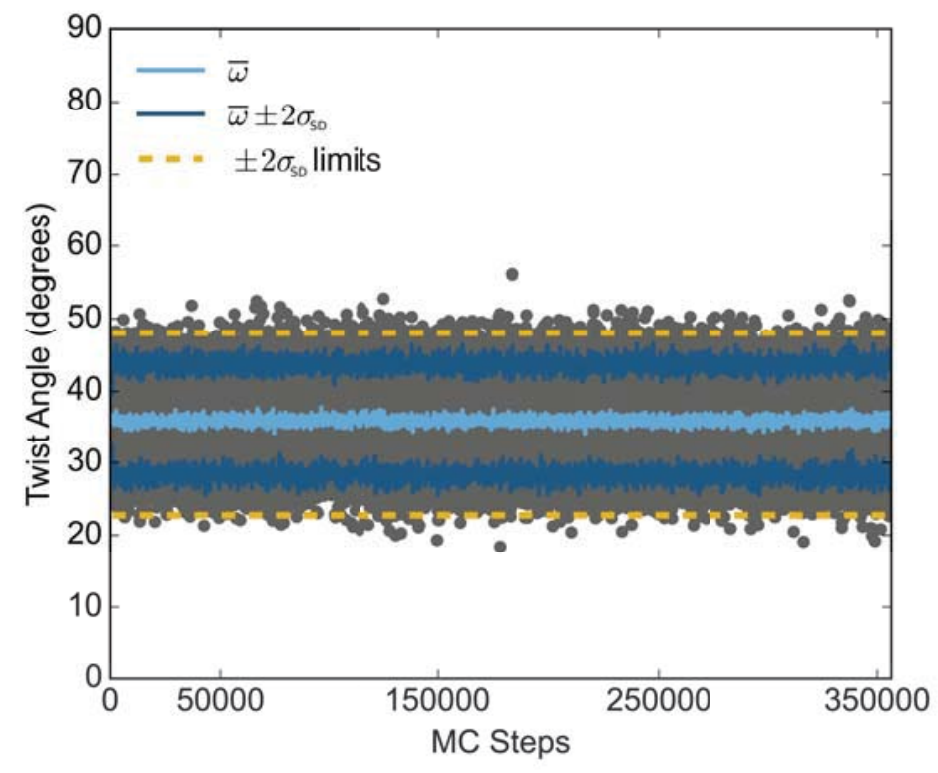

Figure 48. Survey of Simulated DNA Twist Angles: Each gray dots represents the DNA twist angle, $\omega$, as a function of the number of MC twist torsions. For the 58 flexible bp in the $60 \mathrm{bp}$ linear DNA simulated, at each MC step the light blue shows the average twist angle, $\bar{\omega}$, and the dark blue shows two standard deviations, $2 \sigma_{\mathrm{SD}}$, from the mean. Compared to the structure survey from which the energy parameters were obtained [132], the mean twist angle in the simulated structures matches the $+35.4^{\circ}$ while the distribution about the mean is tighter (the yellow lines indicate the $2 \sigma_{\mathrm{SD}}$ limit reported from the structure survey). 
In addition to satisfying DNA bend and twist energetics, the algorithm produces all-atom structures which maintain the canonical structure of B-form DNA despite the intermediate CG step. The essential element to facilitate robust final structures is the energy minimization of structures after the MC process to relieve the strain caused by CG moves. This strain occurs between the $\mathrm{O} 3^{\prime}$ atom on one base and the $\mathrm{P}$ atom of the following base. An ideal mechanism for evaluating the degradation caused by this strain is an analysis of the three dihedral angles which contain the O3'-P bond, $\varepsilon, \zeta$, and $\alpha+1$, illustrated in fig. 49.

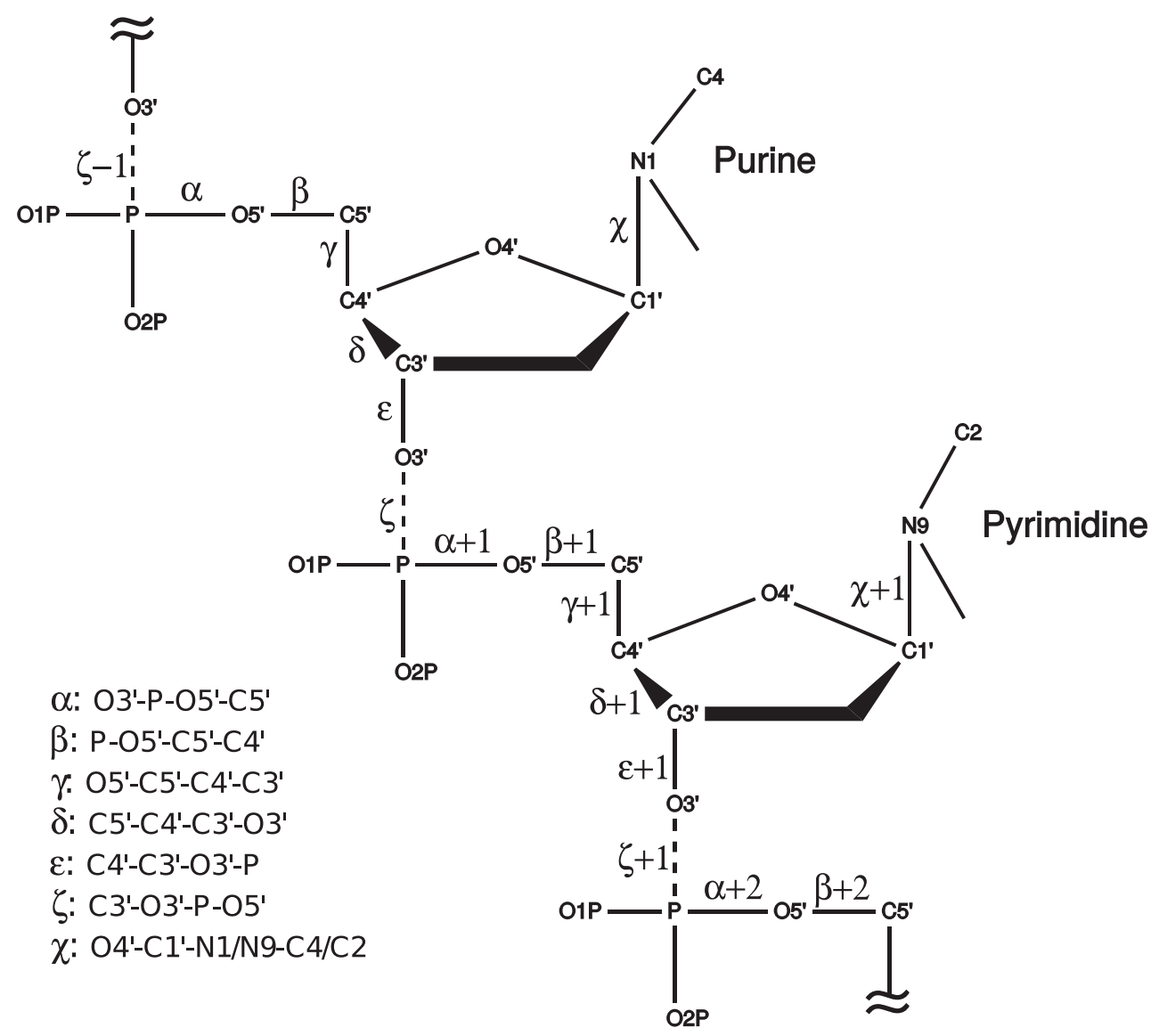

Figure 49. Schematic of a DNA Molecule: DNA has 7 dihedral angles, $\alpha, \beta, \gamma$, $\delta, \varepsilon, \eta$, and $\chi$, defined as the angle between the two planes uniquely described by four sequential atoms. The separation between DNA bases is between the $\mathrm{O}^{\prime}$ atom of one base and the $\mathrm{P}$ atom of the following base. Performing CG MC torsions strains this bond connecting stacked bases (dashed line). This strain causes a distortion of the dihedral angles that include this bond, i.e., $\varepsilon, \zeta$, and $\alpha+1$. A return of these three dihedral angles to be within acceptable ranges indicates the correction of this strain. 
To determine the acceptable ranges for the different dihedral angles we evaluated an MD simulation of a solvated and ionized $12 \mathrm{bp}$ Bform DNA model (PDB ID: 119D [137]). The MD simulation lasted for $75 \mathrm{~ns}$ and used the CHARMM36 force field, which is noted for its improved ability to reproduce the experimentally observed sampling of the different conformations of B-form DNA [138]. Plots of each dihedral angle as a function of time are shown in supplemental figs. 79-85. Table 3 shows the resulting average dihedral angles and their standard deviations. We compare theses dihedral angles from solvation MD simulation with those reported from a survey of 34 B-form DNA crystal structures [136]. We note the consistency between the average angles from MD simulation and crystal structure with a marked increase in standard deviation observed for the simulation
Table 3. Summary of DNA Dihedral Distributions: Dihedral angles from an MD simulation of a solvated and ionized B-form DNA model, compared to those from a collection of 34 B-form DNA crystal structures [136]. The tabulated values show the mean plus or minus one standard deviation. Subscripts differentiate BI and BII conformations (and purine versus pyrimidine for $\left.\chi_{\mathrm{I}}\right)$.

\begin{tabular}{|l|c|c|}
\hline Angle & MD & Crystal \\
\hline$\alpha$ & $300^{\circ} \pm 20^{\circ}$ & $298^{\circ} \pm 15^{\circ}$ \\
\hline$\beta_{\mathrm{I}}$ & $165^{\circ} \pm 24^{\circ}$ & $176^{\circ} \pm 9^{\circ}$ \\
$\beta_{\mathrm{II}}$ & $152^{\circ} \pm 26^{\circ}$ & $146^{\circ} \pm 8^{\circ}$ \\
\hline$\gamma$ & $52^{\circ} \pm 15^{\circ}$ & $48^{\circ} \pm 11^{\circ}$ \\
\hline$\delta_{\mathrm{I}}$ & $131^{\circ} \pm 21^{\circ}$ & $128^{\circ} \pm 13^{\circ}$ \\
$\delta_{\mathrm{II}}$ & $136^{\circ} \pm 13^{\circ}$ & $144^{\circ} \pm 7^{\circ}$ \\
\hline$\varepsilon_{\mathrm{I}}$ & $185^{\circ} \pm 20^{\circ}$ & $184^{\circ} \pm 11^{\circ}$ \\
$\varepsilon_{\mathrm{II}}$ & $247^{\circ} \pm 30^{\circ}$ & $246^{\circ} \pm 15^{\circ}$ \\
\hline$\zeta_{\mathrm{I}}$ & $259^{\circ} \pm 22^{\circ}$ & $265^{\circ} \pm 10^{\circ}$ \\
$\zeta_{\mathrm{II}}$ & $182^{\circ} \pm 37^{\circ}$ & $174^{\circ} \pm 14^{\circ}$ \\
\hline$\chi_{\mathrm{I}_{\mathrm{pur}}}$ & $259^{\circ} \pm 16^{\circ}$ & $258^{\circ} \pm 14^{\circ}$ \\
$\chi_{\mathrm{I}_{\mathrm{pyr}}}$ & $249^{\circ} \pm 22^{\circ}$ & $241^{\circ} \pm 8^{\circ}$ \\
$\chi_{\mathrm{II}}$ & $272^{\circ} \pm 16^{\circ}$ & $271^{\circ} \pm 8^{\circ}$ \\
\hline
\end{tabular}
angles.

To evaluate the structural quality of DNA models generated through our MC algorithm, we surveyed the seven dihedral angles from resulting structures with a particular focus on the angles containing the $\mathrm{O} 3^{\prime}-\mathrm{P}$ bond. For the starting structure, we used a 60 bp linear DNA model generated from a random sequence using the 3DDART DNA structure modeling server [139]. Using NAMD [3] with the CHARMM36 force fields, we prepared this model for simulations by performing 2000 minimization steps followed by $200 \mathrm{MD}$ steps (0.2 ps) then another 2000 minimization steps. After 
this preparation process, all the model dihedral angles were within two standard deviations of the mean values from MD simulations, shown in table 3. A critical step in this preparation process is generating a protein structure file (PSF) containing details on all the bonds in the structure. The bond information in this PSF is required as input for the minimization process. This facilitates minimizing the raw structures after completing MC sampling, as it identifies the coordinates which need to be adjusted in order to resolve bond strain.

With this prepared starting structure, we selected values for $\delta \vartheta_{\max }$ between 1$60^{\circ}$ and performed over 1000 steps per flexible DNA bp, generating over 65000 unique structures for each $\delta \vartheta_{\max }$. For a sample structure generated using 71000 MC steps, fig. 50 compares select scatter plots of DNA dihedral angles, before and after energy minimization, The shaded regions show the angles within two standard deviations of the mean, yellow and red respectively represent BI and BII backbone conformations. Before minimization approximately 20 percent of the $\alpha, \varepsilon$, and $\zeta$ dihedrals are beyond two standard deviations of the mean. After minimization, only 3 percent of these same dihedrals are beyond two standard deviations from the mean, an acceptable amount for a normal distribution.

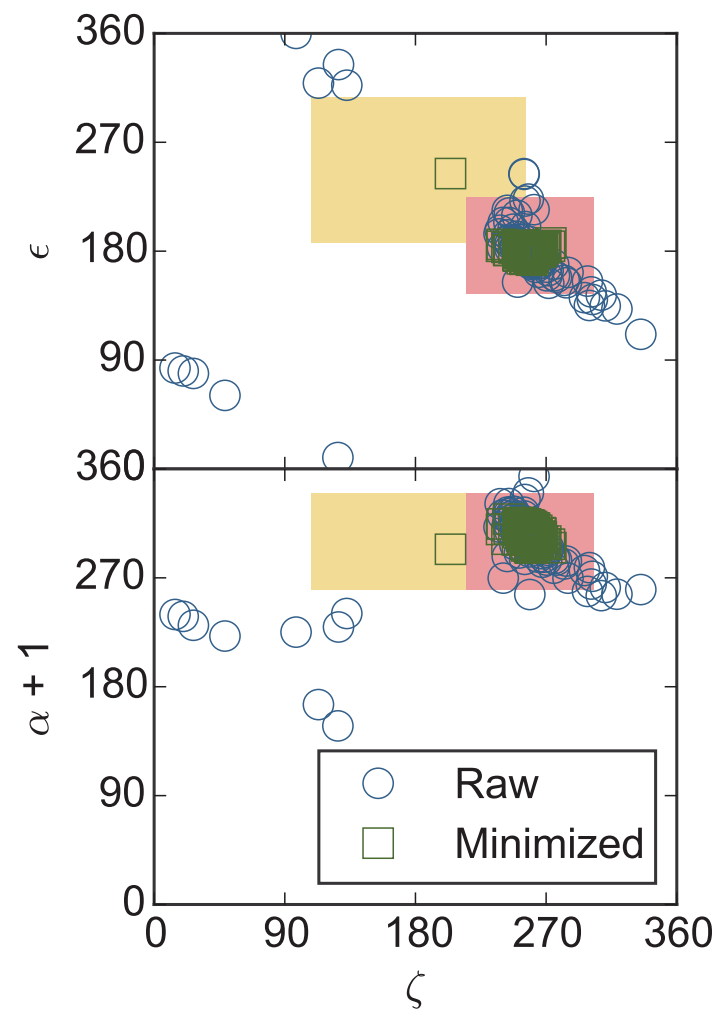

Figure 50. Raw versus Energy Minimized Dihedral Angles: Scatter plots of the DNA dihedral angles between adjacent DNA bps. These angles are from a 60 bp DNA model after each of the 58 flexible bases has experienced an average of $1233 \mathrm{MC}$ steps using a $\delta \vartheta_{\max }=10^{\circ}$. The red and yellow patches respectively show the $2 \sigma_{\mathrm{SD}}$ ranges for $\mathrm{BI}$ and $\mathrm{BII} \mathrm{B}-$ form DNA, based on the MD simulation results (table 3). 
For each $\delta \vartheta_{\max }$ trajectory, we sampled every hundredth structure to evaluate how the percent of dihedral angles within the two standard deviation range changes as a function of the number of $\mathrm{MC}$ steps per bp, both before and after minimization. Figure 51 plots this percentage as a function of the number of MC steps, comparing angles before and after performing 2000 minimization steps. Note that for all three of the dihedrals containing the $\mathrm{O}^{\prime}-\mathrm{P}$ bond, the percent in range, regardless of the $\delta \vartheta_{\max }$ used, are relatively indistinguishable after 200 steps per bp. Additionally, after 2000 minimization steps, for nearly every structure all seven of the dihedral angles are within the $95 \%$ cutoff (gray
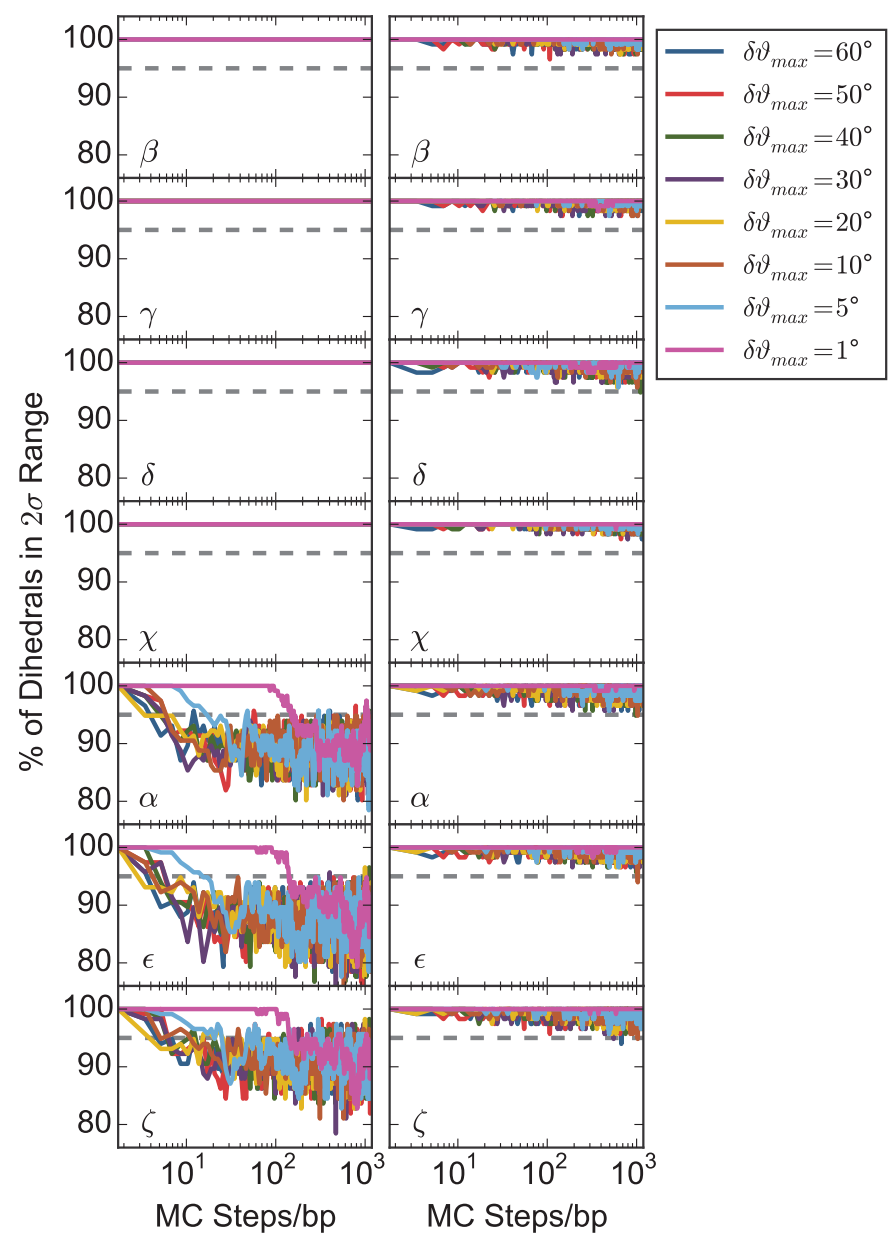

Figure 51. Percent of Acceptable Dihedral Angles: Percent of DNA dihedral angles, before (left) and after (right) energy minimization, that are within 2 standard deviations of the mean values from a $75 \mathrm{~ns}$ MD simulation of DNA, table 3. This percentage is plot as a function of the number of accepted MC steps, normalized by the number of flexible bps. The dashed lines mark the $95 \%$ cutoff for the percent of dihedral angles that should be within the 2 standard deviations of the mean using a normal distribution.

dashed line) for a normal distribution. This demonstrates that energy minimization is able to relax the bond strain between stacked bases even after performing $1000 \mathrm{MC}$ steps per flexible bp, with a maximum $\delta \vartheta \leq 60^{\circ}$.

Exploring repeated minimizations of the same structure, we recognized that vac- 
uum minimization using the CHARMM36 force field leads to degradation of the DNA molecule. Repeating the 2000 step minimization process a hundred times, we visually recognized distortions in the DNA after as few as 10 cycles or 20000 minimization steps. Plotting the dihedral angles within two standard deviations of the mean as a function of the number of minimization iterations, supplemental fig. 86, shows that the dihedral angles do not exceed the limit for a normal distribution but a visual comparison between the minimized and initial structures reveals a collapse of the minor groove after as few as 10 cycles, illustrated as an inset of fig. 86 .

In light of this degradation caused by repeated vacuum minimizations, the recommended protocol to fully sample configuration space, given a valid starting structure, is to generate structures using up to $1000 \mathrm{MC}$ steps per flexible bp then perform a 2000 step minimization on all structures at the conclusion. Parallel simulation trajectories can be performed either using the original starting structure or using a select structure taken from a prior trajectory that has not been minimized. In the latter case, one should reduce the number of MC steps per flexible bp by the number of

Table 4. Summary of Simulation Parameters: The algorithm is available for public use at, https://sassie.web.chem.utk.edu/sassie2/. The web interface allows users to selectively modify both the standard and advanced parameters as desired.

\begin{tabular}{|ll|}
\hline Standard Parameters & Default \\
$\delta \vartheta_{\text {max }}:$ & $10^{\circ}$ \\
Steps before minimizing: & $\leq 1000$ steps per flexible bp \\
Minimization procedure: & 2000 minimization steps \\
\hline Advanced Parameters & Default \\
DNA persistence length $\left(L_{p}\right):$ & $534 \AA$ \\
Twist force constant $(\kappa):$ & $0.062 \times\left(1^{\circ}\right)^{-2}$ \\
\hline Fixed Parameters & \\
Granularity: & $1 \mathrm{bp} / \mathrm{bead}$ \\
Rod length $(\mathrm{l}):$ & $3.38 \AA$ \\
Wormlike chain width $(\sigma):$ & $3.01 \AA$ \\
Mean twist angle $(\omega):$ & $35.4^{\circ}$ \\
\hline
\end{tabular}


steps already performed. A short 2000 step minimization should be performed after completing $\mathrm{MC}$ simulations to relieve the strain caused by $\mathrm{CG}$ torsions. After minimizing, structures that match the data can be studied further using robust MD simulation methods in which the structure is solvated and ionized. Table 4 contains a summary of the simulation parameters discussed, grouping them into either standard input, advanced input, or fixed parameters. Using this protocol as set forth, the MC sampling algorithm provides rapid configuration space coverage allowing one to explore local configuration space using MD.

\section{Examples}

To demonstrate the application of this algorithm, we show two example simulations, the first simulates flexible DNA tails of a single nucleosome, or mononucleosome, and the second simulates flexible linker DNA between an array of four connected nucleosomes, or tetranucleosome. These examples not only exhibit the creation of an ensemble of structures but also filtering that ensemble against mock experimental data.

Nucleosomes are the molecular building block of chromatin. The structure of chromatin and its connection to gene regulation have eluded our understanding to date [5]. In spite of decades of effort to reveal chromatin conformations, the findings in cell nuclei tend to provide more questions than answers due to the complex nature of the problem and results from in vitro studies in solution remain confined to the level of single nucleosomes. It remains an open question whether the regulatory roles of chromatin surpass its biological and chemical (i.e., epigenetic) content. The limited understanding of chromatin structure and conformation beyond the level of single nucleosomes inhibits understanding its regulatory role in a manner similar to how not knowing the molecular structure of an enzyme would prevent gaining a clear understanding of its function. The DNA MC algorithm presented here provides the 
ability to generate an ensemble of structure models, which can be filtered against experimental data then analyzed to obtain this quantitative structure knowledge.

Two areas of active investigation related to chromatin structure question the amount of DNA breathing and unwrapping of mononucleosomes [29, 31] and the configuration of a short array of 2-12 nucleosomes [2, 27, 32]. To demonstrate DNA unwrapping using this algorithm, we performed MC simulations on a mononucleosome model, allowing $30 \mathrm{bp}$ on each end to be flexible (fig. 52 (d) and (e) show two views of a mononucleosome with the designated flexible DNA shown inside the white isosurface). To simulate varied configurations of nucleosome arrays, we performed MC simulations on a tetranucleosome model, allowing each of the three linker DNA fragments to be flexible (in fig. $53(\mathrm{~d})-(\mathrm{k})$, the linker DNA is the DNA between nucleosomes). For both the mononucleosome and tetranucleosome examples, all proteins and any DNA not designated as flexible were considered rigid bodies throughout the simulations. Their atomic coordinates for the starting structures were based on the crystal structures of the 1KX5 mononucleosome [1] and the 1ZBB tetranucleosome [2]. These starting structures were prepared in the same manner as the $60 \mathrm{bp}$ linear DNA model used for the dihedral angle verification.

To create mock experimental data for the comparison of both the mononucleosome and tetranucleosome, we performed a $\mathrm{MC}$ simulation on each initial structure and selected a random resulting structure as the goal molecule. We then calculated the theoretical scattering for the goal structure using FoXS [108].

The discrepancy between the SAXS profiles from the model data, $I_{m}$, and the mock experimental data, $I_{e}$, was quantified using the $R$-factor, defined as

$$
R=\frac{\sum_{i=1}^{N}\left|I_{e}\left(Q_{i}\right)-c_{\text {scale }} I_{m}\left(Q_{i}\right)\right|}{\sum_{i=1}^{N}\left|I_{e}\left(Q_{i}\right)\right|}
$$



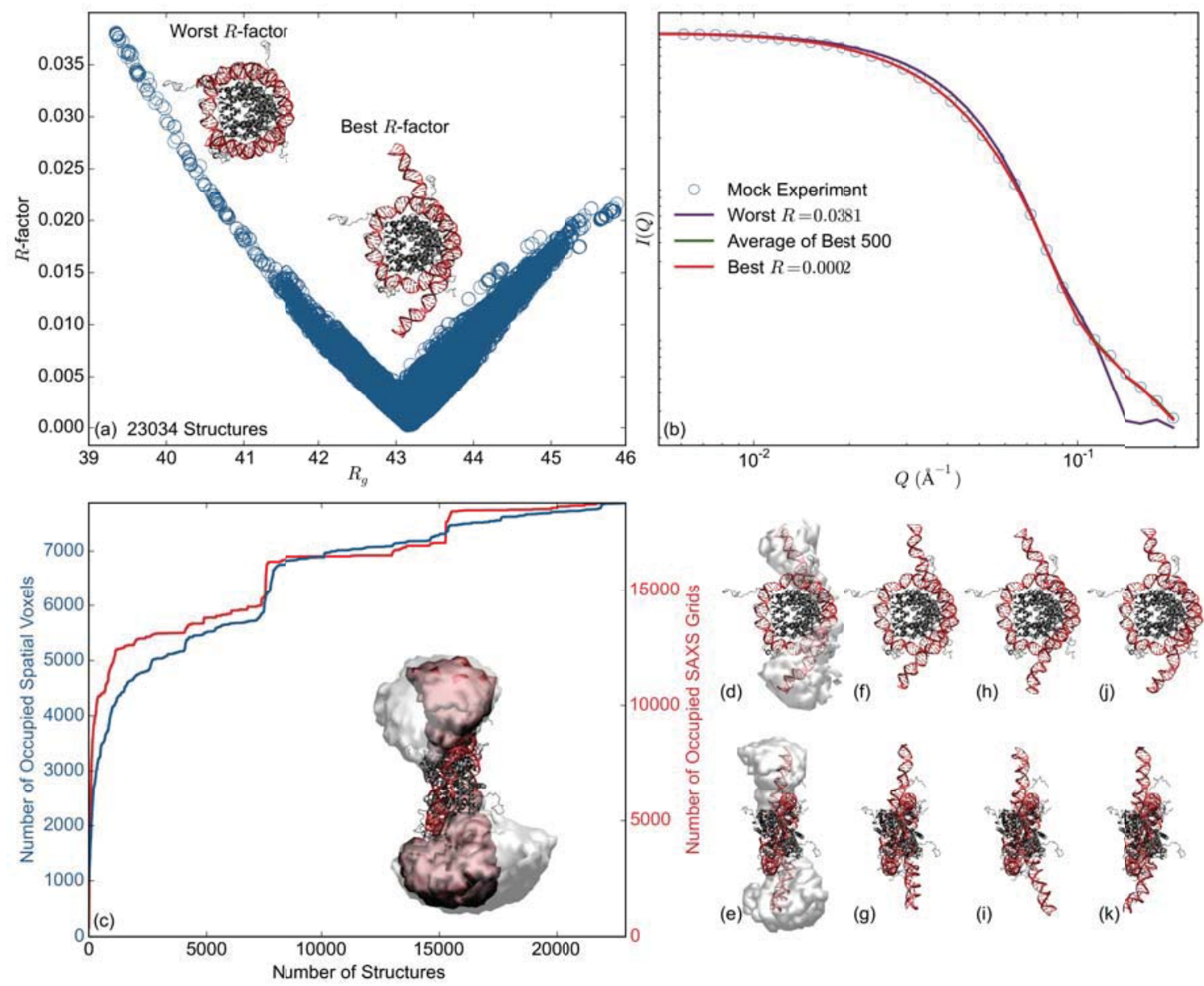

Figure 52. Example Mononucleosome Simulation: An example of filtering a structure ensemble of mononucleosome models against mock experimental SAXS data. (a) $R$-factor versus $R_{g}$ for each structure. Labeled insets illustrate the model structures with the smallest and largest $R$-factor. (b) mock experimental data and the scattering intensity calculated from the worst match (purple), best match (red), and the average of the 500 best matches (green). (c) spatial (blue) and SAXS (red) convergence analysis for the structure ensemble as a function of the number of structures in the ensemble. The inset in (c) shows the spatial range envelope of the entire ensemble of structures (white) overlaid on the envelope for the sub-ensemble of the 500 best-matched structures (red). Panels (d) and (e) show from two angles the spatial range envelope for the best-matched sub-ensemble, overlaid on an illustration of the structure with the smallest $R$-factor. Panels (f)-(k) show three additional example structures from the best-matched sub-ensemble, viewed from the same two angles. The view in (d), (f), (h), and (j) is looking at the nucleosome face. The view in (e), $(\mathrm{g})$, (i), and (k) is looking at the nucleosome dyad. 
summing over $N$ points in $Q$, and scaling the data sets to match at $Q=0$ using the scale factor $c_{\text {scale }}=I_{e}(0) / I_{m}(0)$. We do not use the $\chi^{2}$ statistic as we do not have true estimates of the experimental error.

Figures 52 and 53 respectively show the results from comparing the MC ensemble of 23044 mononucleosome structures and 86001 tetranucleosome structures with their respective experimental data. In each figure, (a) shows a plot of the $R$-factor versus $R_{g}$ for each structure with insets illustrating the structures with the best and worst discrepancy. Panel (b) compares the experimental data with the calculated theoretical scattering from the best-matched, worst-matched, and the 500 best matches. Panel (c) shows a convergence analysis for the structure ensemble in both real space and reciprocal space with an inset comparing the spatial range envelopes from the entire ensemble and best 500 structure sub-ensembles. Panels (d) and (e) show from two angles the spatial range of the best sub-ensemble overlaid on an illustration of the structure with the absolute smallest $R$-factor. This range exemplifies the extent of structures which represent mock experimental data equally well. Panels (f)-(k) further depicts the variance in valid structures by showing three additional example structures randomly selected from the sub-ensemble of best structures.

When creating an ensemble of structures it is important to consider when convergence has been reached, or at what point have a sufficient number of structures been generated to sufficiently sample the configuration space. To quantify the convergence of an ensemble, we analyze each new structure and its calculated scattering to determine how different it is from the other structures in the ensemble, both in real space and the reciprocal space of SAS, as shown in figs. 52(c) and 53(c). For the real space analysis, we discretize space into $5 \AA$ voxels and for each structure count the number of new voxels that are occupied by either an alpha carbon or a phosphate atom. For the reciprocal space analysis, we consider the calculated scattering curve from each structure in the ensemble to find the overall maximum, $I_{\max }\left(Q_{i}\right)$, and min- 
imum, $I_{\min }\left(Q_{i}\right)$, at each $Q$ point. We then discretize the possible $I\left(Q_{i}\right)$ between these extremum into $N_{g}=100$ grids. For each new structure we bin $I(Q)$ using,

$$
N_{g}\left\|\frac{I\left(Q_{i}\right)-I_{\min }\left(Q_{i}\right)}{I_{\max }\left(Q_{i}\right)-I_{\min }\left(Q_{i}\right)}\right\|,
$$

then count the total number of occupied bins. For both methods, as configuration space is fully covered, the number of occupied voxels and grids will plateau indicating the ensemble has fully explored all possible spatial configurations and all $I\left(Q_{i}\right)$ values. In our examples, both the mononucleosome and tetranucleosome ensembles show convergence using the number of structures generated. We further explored the spatial convergence of the individual nucleosomes in the tetranucleosome array, shown in supplemental fig. 88, and found that each nucleosome also demonstrate convergence.

Plots of the spatial range envelopes, shown as insets in panels (c) of figs. 52 and 53, as well as (d), and (e), allow one to visualize the extent in space that is covered by the ensemble of structures. By limiting these envelopes to a sub-ensemble of bestmatched structures, we identify the range covered by the structures which match the experimental data best. Further, we can separately examine regions corresponding to different molecular domains to see at a glance the region of space each occupies. We demonstrate this in the tetranucleosome range envelopes, fig. 53(c), in which we use separate colored regions to represent each individual nucleosome. Figure 87 further illustrates each of these separate regions individually for added clarity.

These examples demonstrate single structures and sub-ensembles of structures that have SAXS profiles which reproduce the mock experimental data. We note that the number of relevant constraints inherent in a SAS profile is largely unknown, but it is generally accepted that the mathematical problem is largely undetermined. Such highly undetermined problems can have an infinite number of solutions [140]. Thus, one should be careful in choosing a single structure, or even a linear combination of 

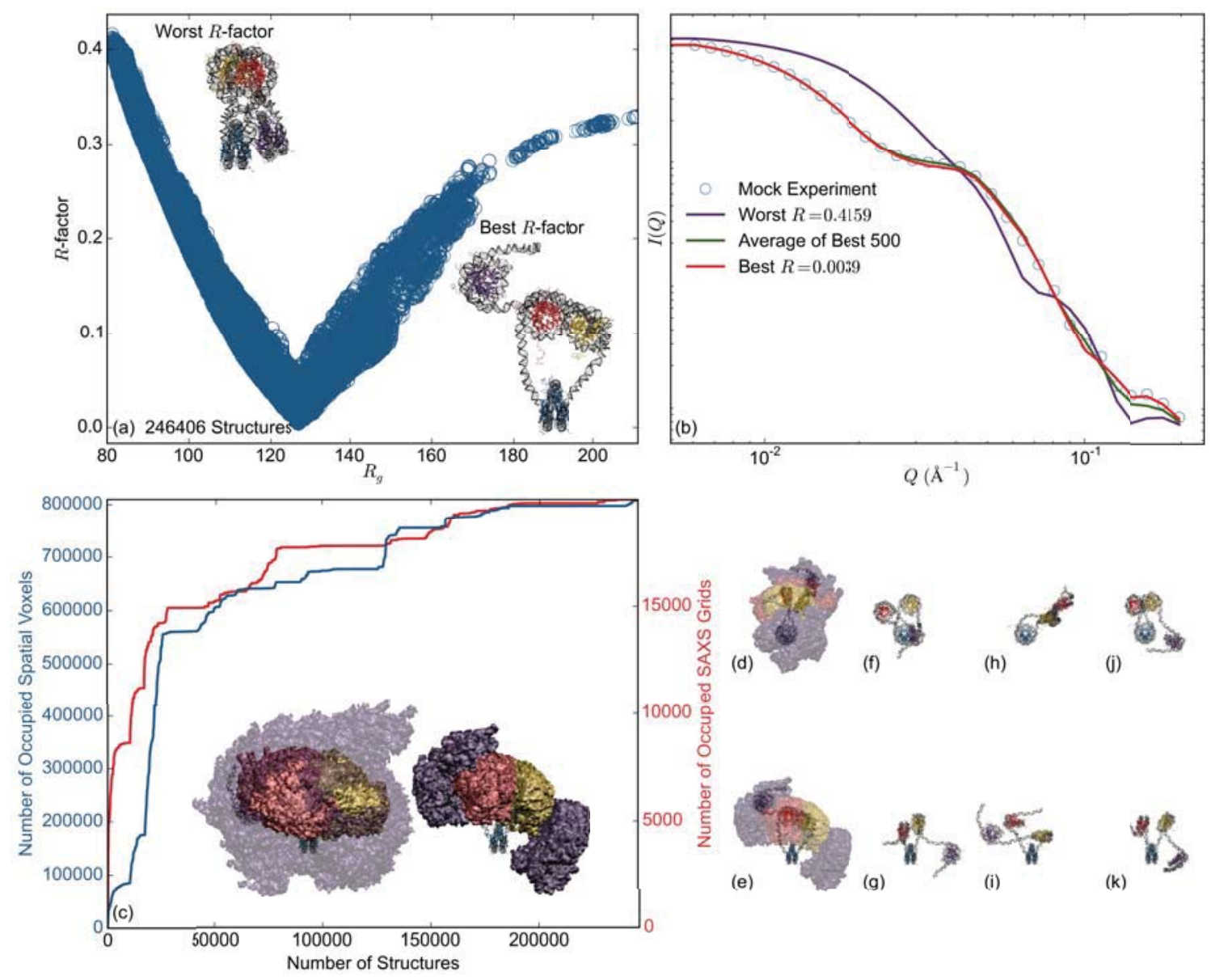

Figure 53. Example Tetranucleosome Simulation: An example of filtering a structure ensemble of tetranucleosome models against mock experimental SAXS data. (a) $R$-factor versus $R_{g}$ for each structure. Labeled insets illustrate the model structures with the smallest and largest $R$-factor. (b) mock experimental data and the scattering intensity calculated from the worst match (purple), best match (red), and the average of the 500 best matches (green). (c) spatial (blue) and SAXS (red) convergence analysis for the structure ensemble as a function of the number of structures in the ensemble. The inset in (c) shows the spatial range envelope of the entire ensemble of structures (left) together with the envelope for the sub-ensemble of the 500 best-matched structures (right). As a reference point, each structure in the ensemble was aligned using the second NCP in the array, illustrated with a blue protein core. The different colored regions indicate the range envelopes for the individual NCPs, where red, yellow, and purple respectively correspond to the range of $\mathrm{NCP}_{1}$, $\mathrm{NCP}_{3}$, and $\mathrm{NCP}_{4}$. Panels (d) and (e) show from two angles the spatial range envelope for the best-matched sub-ensemble, overlaid on an illustration of the structure with the smallest $R$-factor. Panels (f)-(k) show three additional example structures from the 500 best-matched structures. The illustrated proteins are colored to match the spatial ranges shown in (c). The view in $(d),(f),(h)$, and $(\mathrm{j})$ is looking at the $\mathrm{NCP}_{2}$ face The view in (e), (g), (i), and (k) is looking at the $\mathrm{NCP}_{2}$ side. 
structures, as a definitive solution to the problem. We submit that the plots of the spatial range of the best-matched ensemble of structures provide an ideal method for representing the solution structures of molecules which are by their nature dynamic.

\section{Discussion}

We have developed a CG MC algorithm for rapidly generating a large ensemble of atomic molecular structures. The procedure for this algorithm is to represent DNA designated as flexible using a CG wormlike chain model, sample torsions of the CG DNA, recover an all-atom model based on the final CG configuration, then minimize the final structures to relax strain at the boundary between CG beads. The recommended practice for using this algorithm is to use MC sampling to rapidly generate structures spanning all configuration space, perform a short minimization to relax CG effects, then perform robust MD simulations on select structures to explore configuration space at a local level.

Example simulations using this algorithm to explore mononucleosome and tetranucleosome structures demonstrate its ability to generate a large ensemble of structures covering a wide range of configuration space in a reasonable amount of time. Filtering these ensembles using SAXS data provides a reliable means of elucidating the dynamic structure of molecules in solution.

\section{Chapter Summary}

This algorithm far surpasses the functionality and effectiveness of the user-directed rigid body transformations presented in Chapter 3. Where our rigid body moves simply sampled configuration space, this algorithm performs Metropolis MC sampling of a Markov chain, thereby incorporating the physics of the modified DNA while also exploring configuration space. In Chapter 5, we present the structure ensembles generated using this algorithm and filtered against the experimental nucleosome array 
data. Compared to the manual rigid body models, select models from the MC generated ensembles reproduce the experimental scattering with much greater accuracy. 


\section{Chapter 5: Nucleosome Array Structure Modeling}

\section{Chapter Overview}

Implementing the dsDNA MC simulation algorithm, described in Chapter 4, we performed a much more extensive survey of nucleosome array configurations compared to what was possible using user-directed rigid body transformations. Iteratively sampling MC moves of the best-matched structures, we constructed ensembles of hundreds of thousands of atomic nucleosome array structures.

In this chapter we present and discuss the results from filtering these ensembles against the experimental data reported in Chapter 3. We begin by comparing different metrics for ranking models and selecting models best-matched to experimental data. Sorting the structure ensembles using the $R$-factor, we obtain sub-ensembles that describe the experimental scattering for the majority of measured samples. We explore possible reasons for not being able to find model structures to represent some of the experimental data. We conclude by reporting the relative positions and orientations of NCPs in the sub-ensembles of best 1000 structures for the $4 \times 167$ gH5 array data, as select models showed the most favorable agreement to these data sets. Motivated by the resemblance between the theoretical scattering from the 1ZBB model and the scattering from the $4 \times 167 \mathrm{gH} 5$ array in $1 \mathrm{mM} \mathrm{Mg}^{2+}$, we identify distinct differences between the 1ZBB configuration and the average configuration of the experimental sub-ensemble.

\section{Comparing Discrepancy Metrics}

Comparing model and experimental data requires a metric for quantifying model discrepancy. In X-ray crystallography, the $R$-factor has been established universally for qualifying the goodness-of-fit [141]. For SAXS, there is not a well-established metric, rather a multitude of different methods are employed. To identify models 
that best match the data, these various methods weight each $Q$-point differently, thereby selecting different models as the best match to the data.

In addition to weighting points differently, each of these metrics contains a scale factor, $c_{\text {scale }}$, used to superimpose the model data on the experimental results. ${ }^{8}$ One common method for determining this scale factor is to select the value which produces the lowest discrepancy, depending on the metric used. This naive approach focuses on maximizing the quality of the comparison treating all data as equally relevant. As data at a given $Q$-point encodes information specific to a certain size, related by the Bragg equation (17), it is common to filter data using only a limited $Q$-region to specifically explore structures within the corresponding size range [142], e.g., to explore a specific conformational change.

Another method for setting $c_{\text {scale }}$ is to scale the two data sets to match exactly at a designated $Q$-point, typically $Q=0$,

$$
c_{\text {scale }}=\frac{I_{e}(0)}{I_{m}(0)}
$$

where $I_{e}(0)$ and $I_{m}(0)$ are respectively the experimental and model scattering at zero angle. ${ }^{9}$ Recall that $I(0)$ encodes the molecular weight of the scattering molecule. As molecular weight is a fundamental property of any molecule, scaling the model to match the experimental $\mathrm{I}(0)$ enforces that the model accurately describes this quantity. When using all-atom models, the model is built to specifically match the intended molecular composition of the measured sample. If a model were to closely match the measured $I(Q)$ for the majority of the $Q$-range but disagree at $Q=0$, this would represent a discrepancy between the experimental and intended molecular compositions. Additionally, when expanding $I(Q)$ in powers of $Q^{2}$, eq. (10), $\mathrm{I}(0)$ is the zeroth order term in the expansion, representing the most significant factor

\footnotetext{
8 Often this scale factor is not explicitly reported.

${ }^{9}$ For the experimental data, $I_{e}(0)$ is calculated using the Guinier fit (described in Chapter 1).
} 
contributing to the scattering. By choosing to match at this point, we force the first order term in this expansion to match, then look for models which agree with the higher order terms.

The match quality between a model and experimental curve is, therefore, a combination of both the metric used and the scale factor applied to the model data. To illustrate the differences between the four metrics presented by Putnam et al. [141], we show here model comparisons that for consistency have been scaled to match experimental $I(0)$ determined using the Guinier fit. In comparing these metrics, we found that when some models matched the experimental data relatively well over the entire $Q$-range, the different metrics typically identified these same structures, though with different relative rankings. This is important as we expect a well-matched model to be chosen regardless of the metric used. In the case that only a specific $Q$-range could be matched to the experimental data, the model selection varied drastically depending on the metric applied. Though a worthwhile investigation, we do not discuss here the relative consistency between metric selection when applied to models well matched to the data for all $Q$-values. Rather, to focus on the weights applied to different $Q$-regions, we compare an ensemble of structures to an experimental curve that produced poor fits, the $3 \times 167$ array in $100 \mathrm{mM} \mathrm{K}^{+}$, identified as an outlier based on the calculated $R_{g}$ relative to other salt conditions, shown in fig. 25 .

\section{$R$-factor}

The first metric is an extension to the crystallography $R$-factor [111], developed as the SAXS analog with the specific purpose of combining crystal and solution structures. 

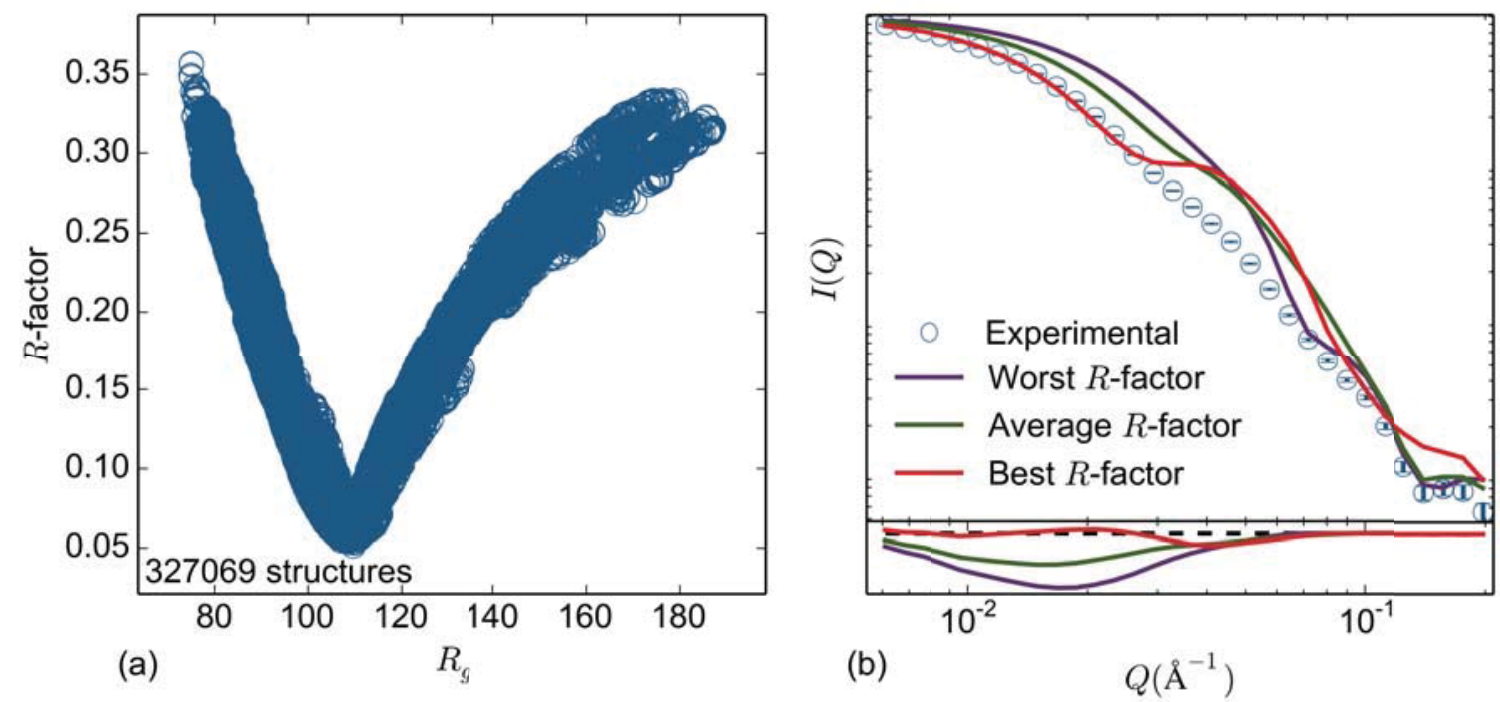

Figure 54. $\quad \mathbf{3} \times \mathbf{1 6 7}$ Ensemble Filtered Using the $\boldsymbol{R}$-factor: (a) $R$-factor for each structure as a function of $R_{g}$. (b) experimental $I(Q)$ compared with the $I(Q)$ calculated from the worst match, best-match, and ensemble average. Comparing to a poorly matched data set exemplifies how the $R$-factor heavily weights the $Q$-range encoding the $R_{g}$.

It is defined as,

$$
R=\frac{\sum_{i=1}^{N_{Q}}\left|I_{e}\left(Q_{i}\right)-c_{\text {scale }} I_{m}\left(Q_{i}\right)\right|}{\sum_{i=1}^{N_{Q}}\left|I_{e}\left(Q_{i}\right)\right|},
$$

where each sum is over $N$ points in $Q$. Though eq. (37) is a fairly simple expression, determining an analytic expression for the value of $c_{\text {scale }}$ which minimizes the $R$ factor is not straight forward because of the absolute values, and therefore must be determined numerically. ${ }^{10}$ Using the $R$-factor to evaluate the comparison between the sample ensemble and data set, using $c_{\text {scale }}$ as defined in eq. (36), fig. 54 illustrates how this metric places strong emphasis on lower $Q$-values, made especially apparent frthe om the distributio; the distribution of angles in broader for tf $R$-factors. From

\footnotetext{
${ }^{10}$ Setting the partial derivative of $R$ with respect to $c_{\text {scale }}$ equal to zero introduces a sign function and cannot be analytically solved for $c_{\text {scale }}, \partial R / \partial c_{\text {scale }}=-\sum I_{m} \operatorname{sgn}\left(I_{e}-c_{\text {scale }} I_{m}\right) / \sum\left|I_{e}\right|$.
} 
the Guinier analysis, this data has an experimental $R_{g}$ of $112.3 \AA$, corresponding to the center of the $V$-shape distribution.

\section{Weighted $R$-factor}

Expanding on this simple metric, Sokolova et al. [142] introduced a weighted $R$-factor, defined as

$$
R_{w}=\frac{\sum_{i=1}^{N_{Q}} Q_{i}^{2}\left[I_{e}\left(Q_{i}\right)-c_{\text {scale }} I_{m}\left(Q_{i}\right)\right]^{2}}{\sum_{i=1}^{N_{Q}} Q_{i}^{2} I_{e}\left(Q_{i}\right) c_{\text {scale }} I_{m}\left(Q_{i}\right)}
$$

where $Q_{i}$ was chosen as a weight factor because it provided the most sensitive criterion. This selection also corresponds to the weight used in the Shannon sampling theorem $[143,144]$. When introduced, the selection of $c_{\text {scale }}$ was set as,

$$
c_{\text {scale }}=\frac{\sum_{i=1}^{N_{Q}} Q_{i}^{2} I_{e}\left(Q_{i}\right) I_{m}\left(Q_{i}\right)}{\sum_{i=1}^{N_{Q}} Q_{i}^{2} I_{m}\left(Q_{i}\right)}
$$

to yield the best least-squares fit. Equation 39 produces a scale factor with units of intensity, $\mathrm{cm}^{-1}$. A consequence of this is that the weighted $R$-factor has units of $\left[(I(Q)-1)^{2} / I(Q)\right]=\left(\mathrm{cm}^{-1}-1\right)^{2} \mathrm{~cm}$. Further, this scaling of the discrepancy metric leads to values for $R_{w}$ that would not be comparable between experiments unless $I(Q)$ is reported on an absolute scale, which is not standard in SAXS.

Rather than scaling the model data to minimize the least-squares fit, one can choose $c_{\text {scale }}$ to instead minimize the weighted $R$-factor. This is achieved by differen- 
tiating eq. (38) with respect to $c_{\text {scale }}$ to obtain,

$$
\frac{\partial R}{\partial c_{\text {scale }}}=\frac{\sum_{i=1}^{N_{Q}} Q_{i}^{2} I_{e}\left(Q_{i}\right)^{2}}{\sum_{i=1}^{N_{Q}} Q_{i}^{2} I_{e}\left(Q_{i}\right) I_{m}\left(Q_{i}\right)}-\frac{\sum_{i=1}^{N_{Q}} Q_{i}^{2} I_{m}\left(Q_{i}\right)^{2}}{c_{\text {scale }}^{2} \sum_{i=1}^{N_{Q}} Q_{i}^{2} I_{e}\left(Q_{i}\right) I_{m}\left(Q_{i}\right)},
$$

from which we see that the minimum weighted $R$-factor occurs when,

$$
c_{\text {scale }}=\sqrt{\frac{\sum_{i=1}^{N_{Q}} Q_{i}^{2} I_{e}\left(Q_{i}\right)^{2}}{\sum_{i=1}^{N_{Q}} Q_{i}^{2} I_{m}\left(Q_{i}\right)^{2}}}
$$

Defined using eq. (41), $c_{\text {scale }}$ is now a unitless value which does not scale with the scattering intensity. Similarly, the resulting $R$-factor is unitless and comparing values
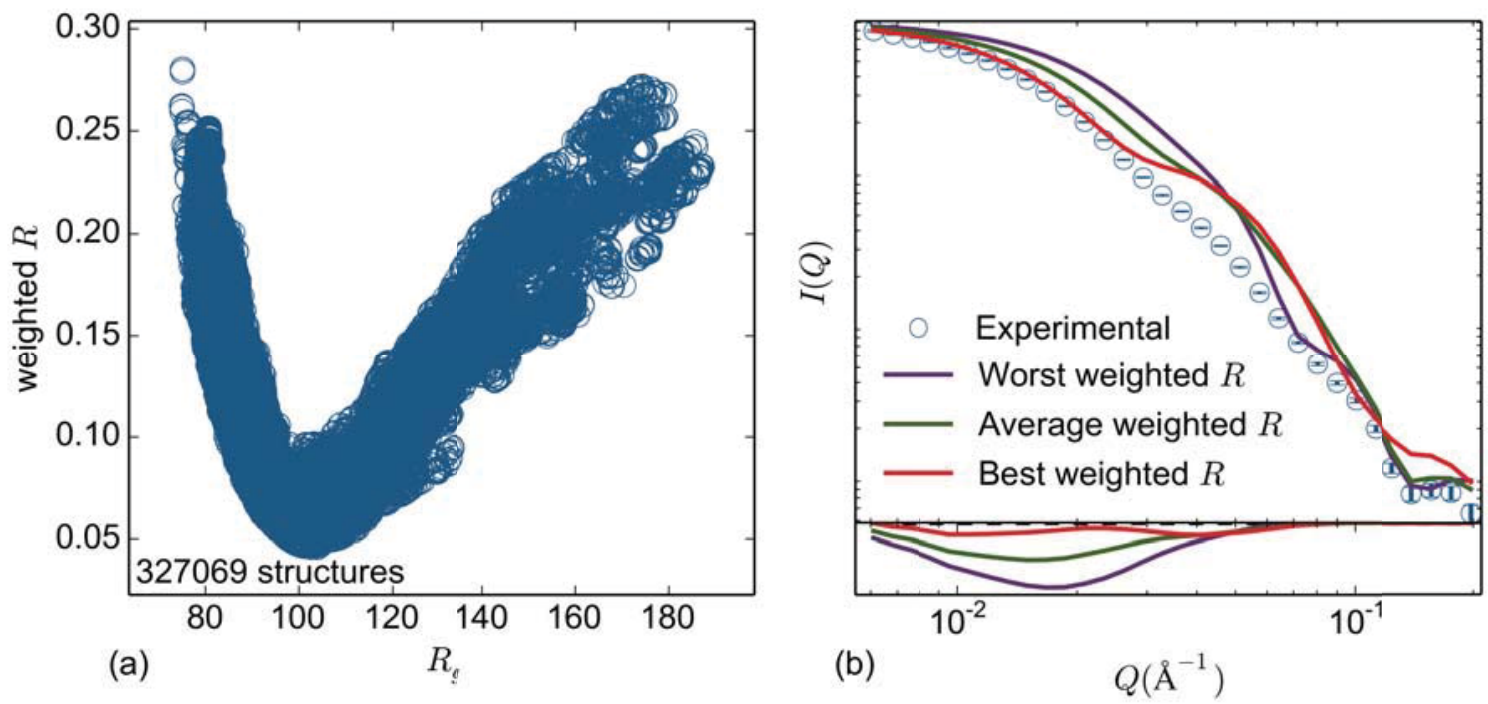

Figure 55. $3 \times 167$ Ensemble Filtered Using the Weighted $\boldsymbol{R}$-factor: (a) weighted $R$-factor for each structure as a function of $R_{g}$. (b) experimental $I(Q)$ compared with the $I(Q)$ calculated from the worst match, best-match, and ensemble average. Comparing to a poorly matched data set demonstrates how the weighted $R$ factor does not emphasize the $Q$-range encoding the $R_{g}$ as strongly than the standard $R$-factor. 
obtained using different experimental setups will be meaningful. We recommend when using this metric, $c_{\text {scale }}$ be defined either using eq. (36), specifically matching the $I(0)$, or eq. (41), to minimize the weighted $R$-factor.

Applying this metric to our example data set and ensemble, with the model data scaled to the experimental I(0), fig. 55 illustrates how the weighted $R$-factor prioritizes structures similar to the standard $R$-factor but with added weight at higher $Q$-values. This difference is most apparent from the increased deviation at $0.009 \AA^{-1}<Q<$ $0.02 \AA^{-1}$ and closer match at $Q>0.02 \AA^{-1}$.

\section{$F$-factor}

As $I(Q)$ spans several orders of magnitude, ${ }^{11}$ a metric termed the $F$-factor,

$$
F=\sqrt{\frac{1}{N} \sum_{i=1}^{N_{Q}}\left[\log \left(I_{e}\left(Q_{i}\right)\right)-\log \left(c_{\text {scale }} I_{m}\left(Q_{i}\right)\right)\right]^{2}}
$$

was introduced to compare the logarithm of the intensities [145]. ${ }^{12}$ This metric was designed to use the $I(0)$ scale factor, though $F$ can be minimized using

$$
c_{\text {scale }}=\exp \left(\sum_{i=1}^{N_{Q}}\left[\log \left(I_{m}\left(Q_{i}\right)\right)-\log \left(I_{e}\left(Q_{i}\right)\right)\right]\right) \text {. }
$$

Figure 56 shows how comparing the logarithm of intensities weights the data at lowest $Q$-values far less than either the $R$-factor or weighted $R$-factor. As a result, the best-matched structure using the $F$-factor depends weakly on the $R_{g}$. For this example, we see in (a), that the best-matched structure has $R_{g} \approx 98 \AA$, compared to the experimental $R_{g}=112.3 \AA$.

\footnotetext{
11 The $3 \times 167$ data shown here spans three orders of magnitude.

12 The $F$-factor was originally used as a fitness factor, where the inverse of eq. (42) was maximized. In either case, it serves to quantify the discrepancy between a model and experimental data.
} 

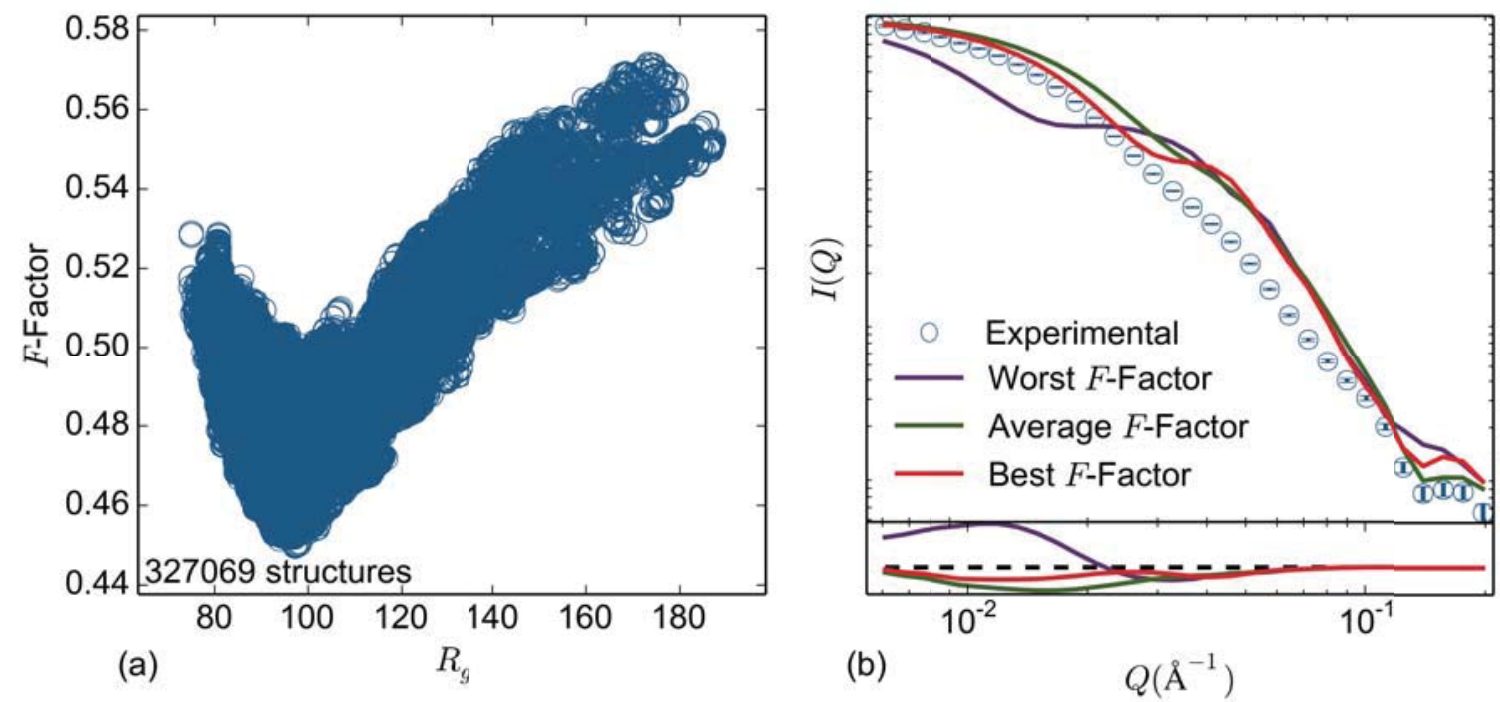

Figure 56. $3 \times \mathbf{1 6 7}$ Ensemble Filtered Using the $\boldsymbol{F}$-factor: (a) $F$-factor for each structure as a function of $R_{g}$. (b) experimental $I(Q)$ compared with the $I(Q)$ calculated from the worst match, best-match, and ensemble average. Comparing to a poorly matched data set illustrates how the $F$-factor weights all $Q$-values relatively evenly, a consequence of including the logarithm.

\section{$\chi^{2}$ Statistic}

Among the different metrics used in SAXS, the reduced chi-squared statistic is the most common. Of the four metrics discussed, this is the only one which weights points according to the experimental error estimates and is defined as,

$$
\chi^{2}=\frac{1}{n-1} \sum_{i=1}^{N_{Q}} \frac{\left[I_{e}\left(Q_{i}\right)-c_{\text {scale }} I_{m}\left(Q_{i}\right)\right]^{2}}{\sigma_{e}\left(Q_{i}\right)^{2}},
$$

where $\sigma_{e}\left(Q_{i}\right)$ is the experimental error estimate at the point $Q_{i}$, and $n$ is the number of degrees of freedom. Similar to the weighted $R$-factor, through differentiation we obtain the $c_{\text {scale }}$ which minimizes the metric,

$$
c_{\text {scale }}=\frac{\sum_{i=1}^{N_{Q}} \frac{I_{e}\left(Q_{i}\right) I_{m}\left(Q_{i}\right)}{\sigma_{e}\left(Q_{i}\right)^{2}}}{\sum_{i=1}^{N_{Q}} \frac{I_{m}\left(Q_{i}\right)^{2}}{\sigma_{e}\left(Q_{i}\right)^{2}}} .
$$



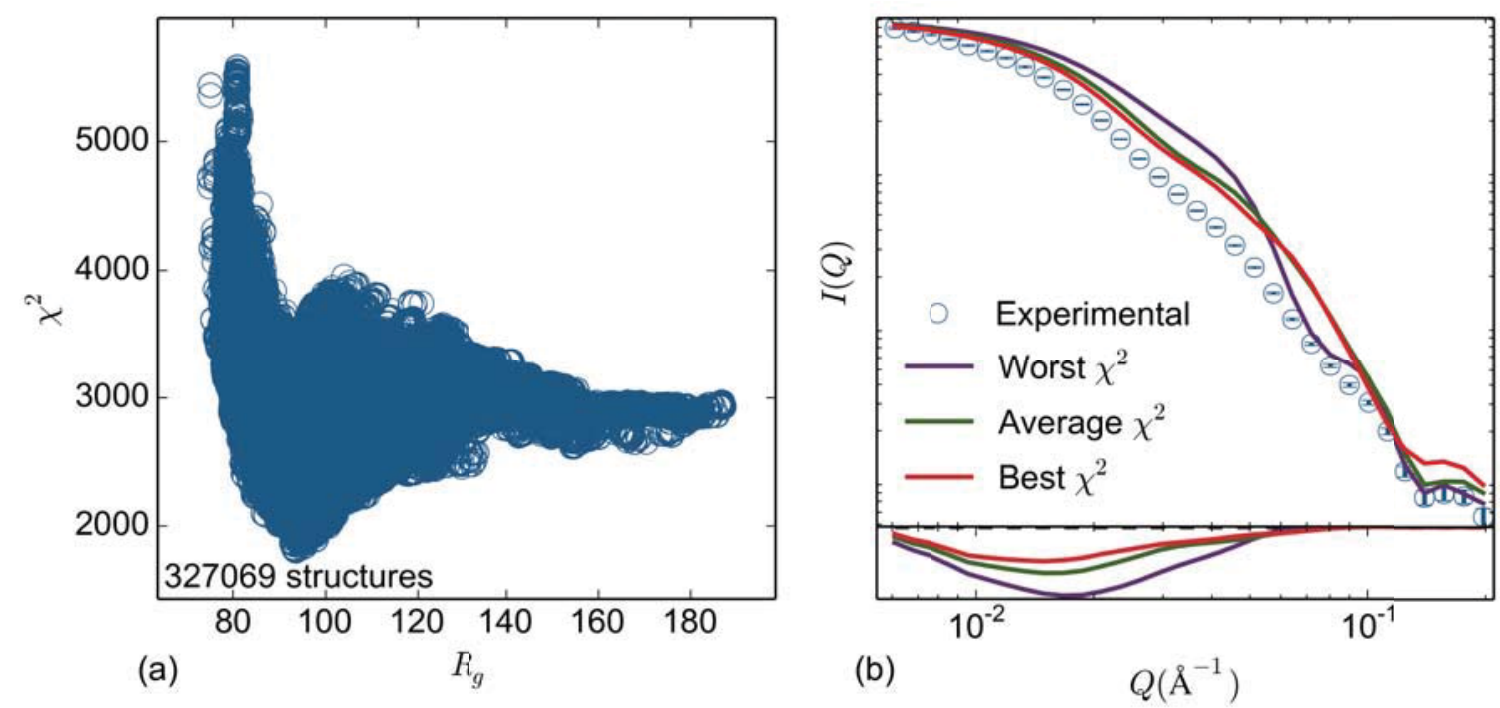

Figure 57. $3 \times 167$ Ensemble Filtered Using the Weighted $\chi^{2}$ Statistic: (a) $\chi^{2}$ statistic for each structure as a function of $R_{g}$. (b) experimental $I(Q)$ compared with the $I(Q)$ calculated from the worst match, best-match, and ensemble average. Comparing to a poorly matched data set exemplifies how the $\chi^{2}$ statistic emphasizes each $Q$-value depending on the ratio between the data and the error estimate, $I_{e}\left(Q_{i}\right) / \sigma_{e}\left(Q_{i}\right)$.

Figure 57 shows that result of filtering models scaled to match the experimental $\mathrm{I}(0)$. We see the model with the lowest $\chi^{2}$ seems to match the data more evenly over the entire $Q$-range when compared to the other three metrics. This is caused by emphasizing data points based on their error estimates, giving the most the weight to data points with the smallest error relative to the measured value, i.e., $\min \left\{\sigma_{e}\left(Q_{i}\right) / I_{e}\left(Q_{i}\right)\right\}$

The $\chi^{2}$ metric originates in probability theory and as the most commonly used metric for comparing models to SAXS data we discuss it here in more detail. Taylor's text, An Introduction to Error Analysis [146], provides a simple but thorough explanation of this $\chi^{2}$ statistic, summarized here with a focus on SAXS data. A key component of the $\chi^{2}$ statistic, defined in eq. (44), is the number of degrees of freedom. In statistical calculations, the number of degrees of freedom is defined as the number of observed data bins minus the number of parameters computed from the data to be used in the calculation. The number of degrees of freedom in SAXS data can be 
estimated by the number of Shannon channels [143, 144] as,

$$
n=\frac{\left(Q_{\max }-Q_{\min }\right) D_{\max }}{\pi}
$$

where $Q_{\min }$ and $Q_{\max }$ are the smallest and largest $Q$ values used in the $\chi^{2}$ comparison and $D_{\max }$ is the maximal distance used in the Fourier transform to obtain $P(r)$ from $I(Q)$, eq. (15). This represents the number of independent $I(Q)$ values and has been shown to be equivalent to the number of independent data points in the real space $P(r)$ [147]. Typically SAXS data is collected on much finer grid than the number of independent channels, often using $N_{Q} \approx 500$, and should consequently be rebinned to a coarser grid before applying the $\chi^{2}$ statistic.

It is important to note that rebinning data with error estimates to a coarser grid is not the same as interpolating the data. The $I\left(Q_{i}\right)$, and especially the $\sigma\left(Q_{i}\right)$, on the new coarse grid should fully represent all points that were combined into that new bin. This is completely different from interpolating, which performs an average, or a weighted average, of the points closest to the new point. To avoid information loss, this binning to a coarse grid is best performed during the initial data reduction, described in the section of Chapter 3 titled, Data Reduction and Buffer Subtraction. When this is not possible, one should combine the errors of the binned points in quadrature, either under the assumption that the number of pixels which contributed to each fine $Q$-bin was the same, or if known, using the number of pixels that contributed to each fine $Q$-bin. ${ }^{13}$

Using data sampled only at each independent point, a well-matched model will fall within the experimental distribution $I\left(Q_{i}\right) \pm \sigma\left(Q_{i}\right)$, and each $Q_{i}$-point will contribute approximately 1 to the $\chi^{2}$ sum. Thus when normalized by the number of degrees of freedom, or the number of independent points, $\chi^{2} \leq 1$ indicates the model is

\footnotetext{
13 We do not go into the details of this here though Ken Tatebe has a report documenting both cases currently hosted at http://isi.ssl.berkeley.edu/ tatebe/whitepapers/ Combining\%20Errors.pdf.
} 
well within the distribution defined by the data. If instead $\chi^{2} \gg 1$ we have strong reason to conclude that the experiment disagrees with the model, or at least that the distribution defined by $\sigma(Q)$ is not a reasonable distribution of the expected $I(Q)$. This depends heavily on both the reliability of the uncertainty values and also correctly determining the number of degrees of freedom.

Once we obtain an observed chi-squared, $\chi_{o}^{2}$, the typical method for rejecting a model is by determining the probability of getting a $\chi^{2}$ as large or larger than $\chi_{o}^{2}$ using,

$$
P_{n}\left(\chi^{2} \geq \chi_{o}^{2}\right)=\frac{2}{2^{n / 2} \Gamma(n / 2)} \int_{\chi_{0}}^{\infty} \chi^{n-1} e^{-\chi^{2} / 2} d \chi,
$$

where $\Gamma(n)=(n-1)$ !. Those using the $\chi^{2}$ metric must compare the resting probability against a chosen threshold probability, beyond which models are classified as not agreeing with the data.

For SAXS data, the typical number of independent degrees of freedom does not exceed 10-15 and accepted structures with a $\chi_{o}^{2}<3$ are common [141]. This means that, assuming $I\left(Q_{i}\right) \pm \sigma\left(Q_{i}\right)$ accurately represents the expected distribution, those using the $\chi^{2}$ metric for SAXS data set their threshold probability for acceptance, eq. (47), at a $P_{n=10}=0.1 \%$ and $P_{n=15}<0.05 \%$ [146]. In other words, this means results are reported that have a $0.1 \%$ or less than $0.05 \%$ confidence level for $n=10$ and $n=15$ respectively. The motivation for accepting model structures with such an unlikely $\chi^{2}$ likely results from a reasonable visual fit, or " $\chi$-by-eye", despite the high $\chi_{o}^{2}$ value.

It is not clear why data with a reasonable visual fit often produce such poor statistical matches. It could be in part because $I(Q)$ spans several orders of magnitude, a consequence of the scattering magnitude decreasing so significantly for increased scattering angles. Another possibility is that the error estimates for SAXS are under- 
estimated, despite following the steps we described in Chapter 3 to obtain reasonable estimates. This would mean that $I(Q) \pm \sigma(Q)$ does not provide an accurate representation of the expected distribution. Some argue that the information content in SAXS data is higher than predicted from the number of Shannon channels [148], and therefore $\chi^{2}$ should be sampled on a finer grid and reduced by an increased $n$. Others disregard the fact that there are so few independent data channels, as many analysis algorithms that calculate the $\chi^{2}$ metric $[108,133,149]$ are regularly used to compare data on an oversampled grid then normalized by the number of data points, artificially reducing their $\chi^{2}$. In this situation, not only is the data normalized by a spurious $n$, but the match quality of points become interdependent and potentially weight the true data channels unevenly.

\section{Motivation for Selecting the $R$-factor}

Despite $\chi^{2}$ being the most commonly used discrepancy metric, owing to the ambiguity surrounding the statistic in relation to SAXS, we choose to instead filter model structures using the $R$-factor. Our primary purpose for using a discrepancy metric is to identify well-matched structures and not to identify the probability a given model represents the data. While we would like to report these probabilities, it is difficult to have confidence in them without first resolving the issues behind the calculation. The $R$-factor is an appealing alternative because of its ability to reproduce the experimental $R_{g}$ as well as its prevalence among the closely related field of crystallography.

The importance of the discrepancy metric prioritizing models which match the $R_{g}$ is best understood in light of eq. (10), in which $I(Q)$ is written as a Taylor series in

powers of $Q^{2}$. In this expansion, $\mathrm{I}(0)$ is the zeroth order term and $-I(0) Q^{2} R_{g}^{2} / 3$ is the first order term. When we force the zeroth order term to match, by scaling to the experimental $\mathrm{I}(0)$, the $R_{g}$ term is the first term we actually compare. If a model were to match the experimental data at higher $Q$-values but have a different $R_{g}$, this would 
indicate the model accurately describes the higher order terms in the expansion but does not agree with the most significant term that was not forced to match.

While the weighted $R$-factor, $F$-factor, and $\chi^{2}$ statistic each have their merits, the decreased priority they afford to models which reproduce the experimental $R_{g}$ makes them a poor choice for filtering all-atom models designed to fully represent experimental data. Panel (a) in figs. 56 and 57 make it especially clear that the $F$-factor and $\chi^{2}$ statistic do not strongly weight the $Q$-range which encodes the $R_{g}$. In addition to ignoring the first term in the Taylor expansion, when generating new model structures using an algorithm which performs small MC steps, as is the case for the algorithm described in Chapter 4, it typically requires several steps to significantly change the $R_{g} \cdot{ }^{14}$ This means that when iteratively generating new structures using the previous best-matched structure, the further the starting structure is from the experimental $R_{g}$, the longer it will take to reach that $R_{g}$. On the other hand, by selecting and sampling structures which match the experimental $R_{g}$, the first structure in each trajectory will have an overall size that matches the experimental system and subsequent structures will probe modification of that structure. This will more rapidly explore the $R_{g}$ region of conformation space best-matched to the experimental data.

\section{Best Model Results}

Implementing the DNA algorithm described in Chapter 4, we generated ensembles hundreds of thousands of $2 \times 167,3 \times 167$, and $4 \times 167$ structures. The starting structures for each of these was based on the 1ZBB structure [2], modified using PSFGEN [3] to match the designed experimental nucleosome array constructs. For the $3 \times 167$ and $2 \times 167$ structures, the atomic coordinates were respectively taken from the first 3 and first 2 nucleosomes from the $4 \times 167$ crystal structure (where the first nucleo-

\footnotetext{
${ }^{14}$ Depending on structure geometry and the maximum step size, $\delta \vartheta$, a single move can drastically change the $R_{g}$. Regardless, using a starting structure with an $R_{g}$ close to the experimental $R_{g}$ will increase the likelihood of generating additional structures with a similar $R_{g}$.
} 
some is closest to the $5^{\prime}$ end of the template DNA). After PSFGEN modifications, the starting structures matched the exact residue content for the each experimental array constructs, the $4 \times 167$ arrays with gH5 being the only exception. Filtering the generated structure ensembles against the experimental data from Chapter 3, we obtain the following results.

\section{$4 \times 167$ gH5 Models}

Of the different experimental data sets, the $4 \times 167$ data with gH5 linker was represented best by model structures generated through the MC DNA algorithm. Shown in fig. 58, the best-matched models reproduce the scattering from the $10 \mathrm{mM} \mathrm{K} \mathrm{K}^{+}$ and $1 \mathrm{mM} \mathrm{Mg}^{2+}$ data over the entire $Q$-range. The lowest $R$-factors for the $10 \mathrm{mM}$ $\mathrm{K}^{+}$and $1 \mathrm{mM} \mathrm{MgCl}_{2}$ are respectively $4.5 \times 10^{-4}$ and $2.8 \times 10^{-3}$, compared to the best $\mathrm{R}$ values from rigid body modeling, $7.8 \times 10^{-2}$ and $3.1 \times 10^{-3}$. More important than the improved $R$-factors, models generated through the MC algorithm match the experimental data over the entire $Q$-range which was not the case for rigid body modeling. We note these close matches are in spite of the lack of gH5 proteins.

We did not perform separate simulations using a $4 \times 167$ gH5 model because of limited time and computing resources. Including the four gH5 proteins adds a total of 2076 heavy atoms (not hydrogen) and constitutes a $0.8 \%$ increase in mass from the $4 \times 167$ array. ${ }^{15}$ While producing a small effect in the calculated scattering around $Q=0.07 \AA^{-1}$ and the $I(0)$, illustrated in fig. 34, this difference would only contribute a small amount to the $R$-factor. We therefore show these comparisons to the $4 \times 167$ structures as a reasonable first step. Resources permitting, we intend to add the missing gH5 proteins into the ensemble of final structures, or a select sub-ensemble, by aligning them into place then repeating the FoXS calculations and comparative analysis.

\footnotetext{
15 The $4 \times 167$ model has 106992 total atoms, 58791 heavy atoms, and an atomic mass of 812952 Da.
} 

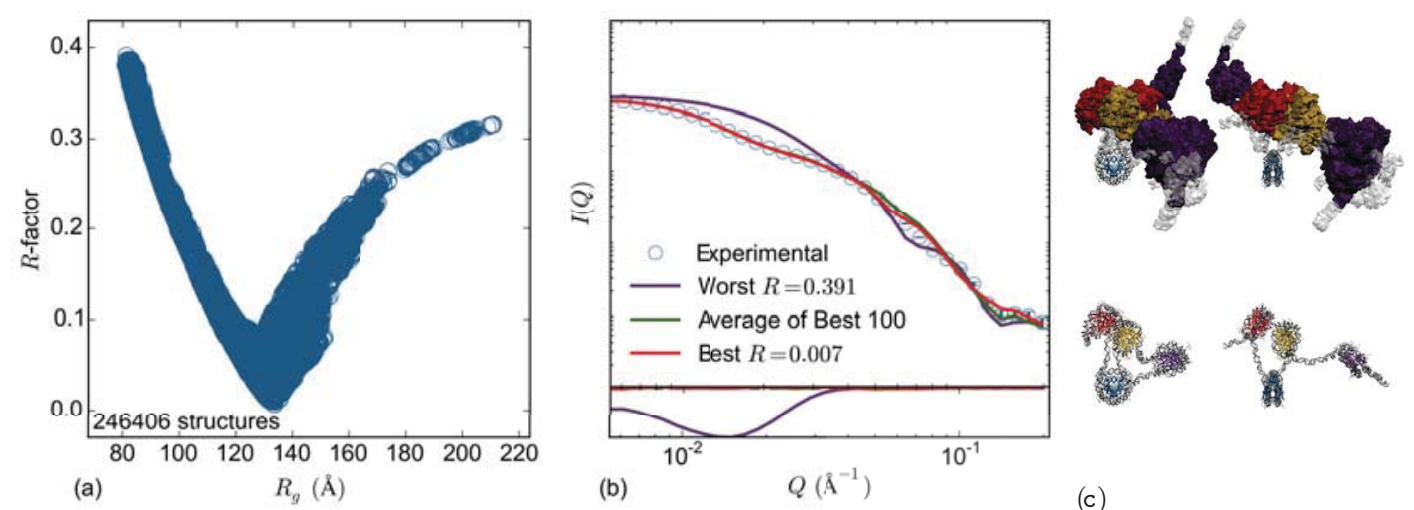

(b)

$Q\left(\AA^{-1}\right)$

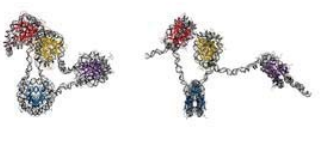

(i) $10 \mathrm{mM} \mathrm{K}^{+}$
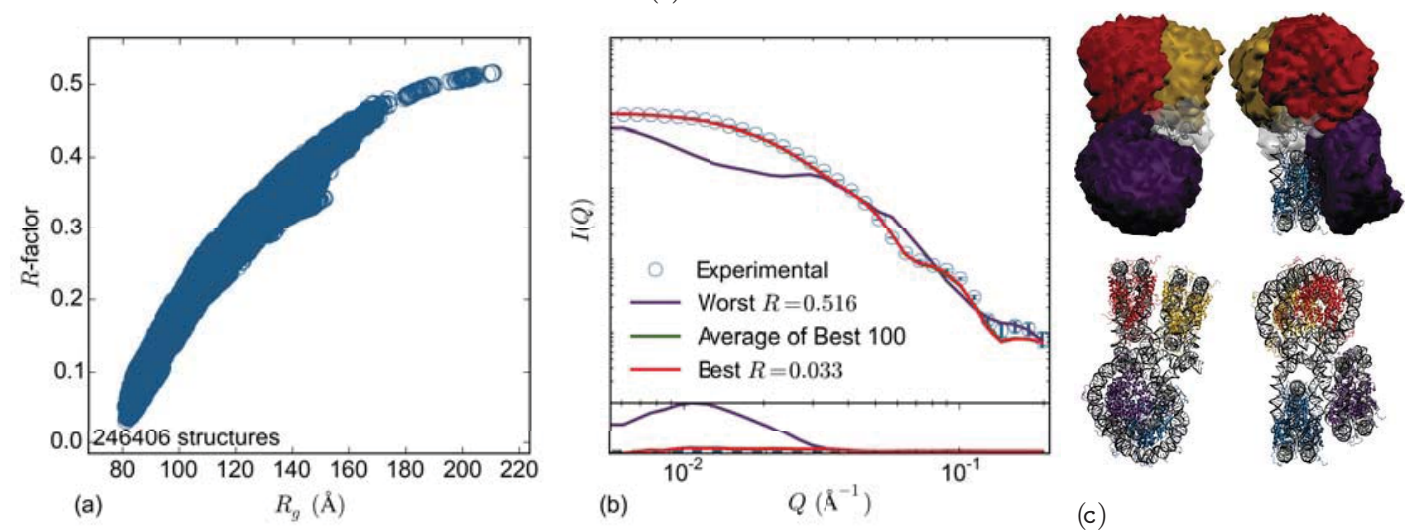

(c)

(ii) $1 \mathrm{mM} \mathrm{Mg} \mathrm{Mg}^{2+}$

Figure 58. $4 \times 167$ Sub-Ensembles for the $10 \mathrm{mM} \mathrm{K} \mathrm{K}^{+}$and $1 \mathrm{mM} \mathrm{Mg}^{2+}$ $4 \times 167$ gH5 Data: (a) $R$-factors versus $R_{g}$ for each structure from the ensemble. (b) experimental $I(Q)$ compared with the $I(Q)$ calculated from the structures with the worst match, the best match, and the average of the 100 best matches; residuals are shown below. (c) two views of the range covered by the best 100 structures (top) with an illustration of the best-matched structures (bottom, animated in the electronic version); the two views are oriented showing the face (left) and side (right) of $\mathrm{NCP}_{2}$. The range of each nucleosome and the nucleosome proteins are colored red, blue, yellow, and purple respectively for nucleosomes 1, 2, 3, and 4. For improved visibility, all structures were aligned using nucleosome 2 (blue) with the nucleosome dyad oriented vertically.

From the range illustrations and example structures shown, panel (c) of fig. 58 (i) and (ii), we appropriately see extended and compact structures matching the $R_{g}$ calculations. For the $10 \mathrm{mM} \mathrm{K}^{+}$, the best-matched structures show three nucleosomes configured similarly to their position in the $1 \mathrm{ZBB}$ structure with one nucleosome significantly extended. The increased ionic screening of the $1 \mathrm{mM} \mathrm{Mg}^{2+}$, clearly pro- 
duces packed structures which resemble the a loosely packed version of the 1ZBB structure. Deviations from the 1ZBB structure are primarily small increases in the opening angle and sliding of stacked nucleosomes. There is not a significant change in the twisting between adjacent nucleosomes.

\section{$4 \times 167$ Models}

Data from $4 \times 167$ arrays without the gH5 linker show a reasonable match to select model structures at low and high $Q$-values, figs. 59 and 60 , but only the $10 \mathrm{mM} \mathrm{K} \mathrm{K}^{+}$ data, fig. 59(i), matches model structures in the intermediate region of $0.03 \AA^{-1}<$ $Q<0.8 \AA^{-1}$. Despite not matching the entire $Q$-range for higher [salts], we discuss features seen in these structures and possible sources for the discrepancy.

Overall, the best-matched structures show much more disorder without the added structural support of the gH5 linker. The $10 \mathrm{mM} \mathrm{\textrm {K } ^ { + }}$ results, fig. 59(i), essentially show that compared to the $1 \mathrm{ZBB}$ structure $\mathrm{NCP}_{1}$ (red) and $\mathrm{NCP}_{3}$ (yellow) slid along their stack plane while $\mathrm{NCP}_{4}$ (purple) has been significantly extended. It is somewhat surprising, considering electrostatic repulsion, that $\mathrm{NCP}_{4}$ and $\mathrm{NCP}_{1}$ end up relatively close together. This is an interesting commonality observed in the best matched $4 \times 167$ structures under all salt conditions, that $\mathrm{NCP}_{1}$ is positioned between $\mathrm{NCP}_{3}$ and $\mathrm{NCP}_{4}$. Because of symmetry we also expect structures to match that are oriented inverse to this, $\mathrm{NCP}_{4}$ positioned between $\mathrm{NCP}_{2}$ and $\mathrm{NCP}_{1}$. Such structures not being included seems to indicate that the 200000 structures generated do not fully explore all these symmetric permutations. ${ }^{16}$ The size and complexity of this system may warrant more extended simulations.

Considering the relative orientation of $\mathrm{NCP}_{1}$ (red) and $\mathrm{NCP}_{3}$ (yellow) in the best matched $50 \mathrm{mM} \mathrm{K}^{+}$results, fig. 59(ii), we observe all three possibilities, face-on-face, side-on-side, and side-on-face. Face-on-face is the most compact orientation, seen in

\footnotetext{
${ }^{16}$ We considered if this was a consequence of aligning to $\mathrm{NCP}_{2}$ but it did not seem to be the case.
} 

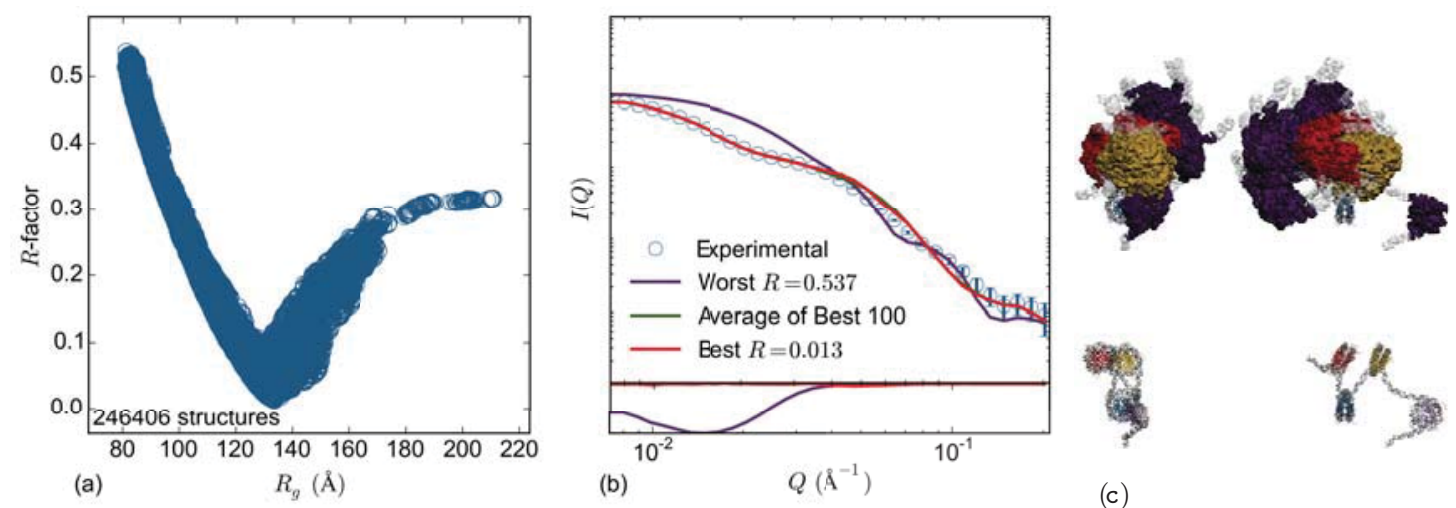

(b)

$Q\left(\AA^{-1}\right)$

(c)

(i) $10 \mathrm{mM} \mathrm{K}^{+}$
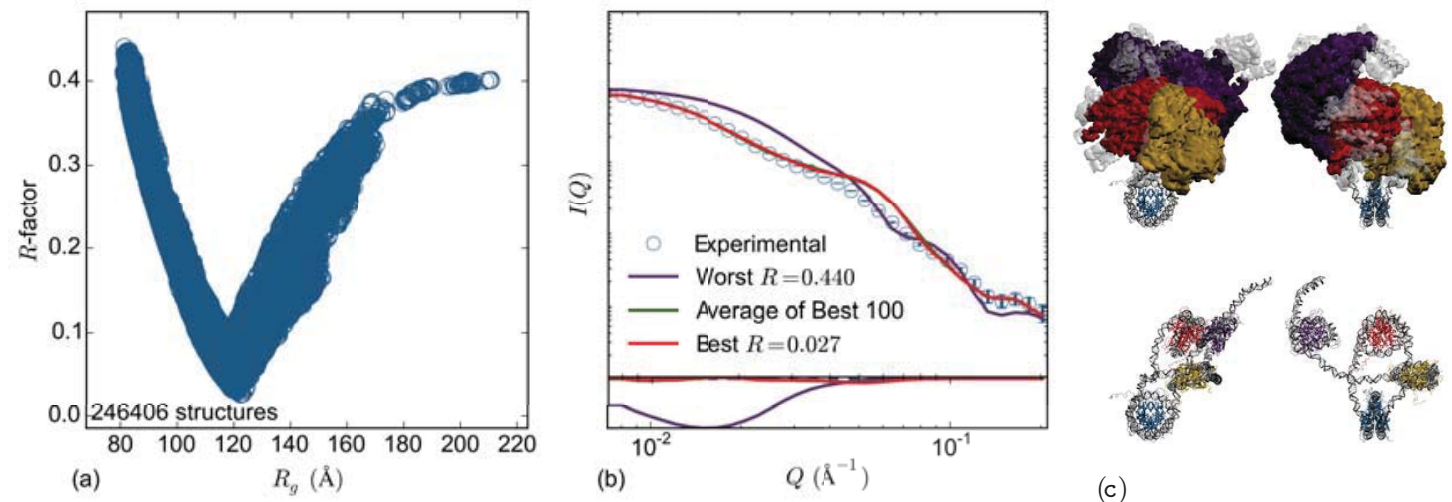

(c)

(ii) $50 \mathrm{mM} \mathrm{K}^{+}$

Figure 59. $4 \times 167$ Sub-Ensembles for the 10 and $50 \mathrm{mM} \mathrm{K} \mathbf{K}^{+}$Data: (a)-(c) as in fig. 58, namely: (a) $R$-factors versus $R_{g}$ for each structure from the ensemble. (b) experimental $I(Q)$ compared with the $I(Q)$ calculated from the structures with the worst match, the best match, and the average of the 100 best matches; residuals are shown below. (c) two views of the range covered by the best 100 structures (top) with an illustration of the best-matched structures (bottom, animated in the electronic version); the two views are oriented showing the face (left) and side (right) of $\mathrm{NCP}_{2}$. The nucleosome (range) and nucleosome proteins (illustration) are colored red, blue, yellow, and purple respectively for nucleosomes 1, 2, 3, and 4. For improved visibility, all structures were aligned using nucleosome 2 (blue) with the nucleosome dyad oriented vertically.

the stacked NCPs of the 1ZBB model, resembling two stacked pancakes. Side-onside stacking is the least compact orientation and resembles colliding hockey pucks. Because it produces larger structures, it is not surprising this is the orientation $\mathrm{NCP}_{1}$, $\mathrm{NCP}_{3}$, and $\mathrm{NCP}_{4}$ (purple) for the $10 \mathrm{mM} \mathrm{K}^{+}$models. Side-on-face stacking orients NCPs orthogonal to each other placing the DNA of one NCP close to the protein 

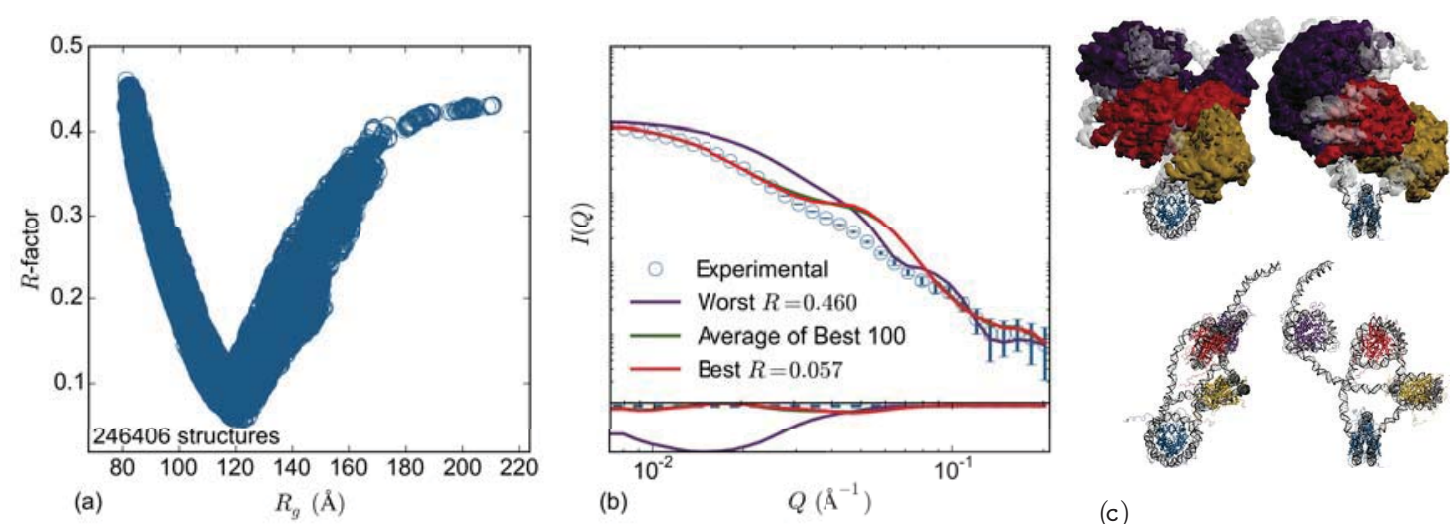

(i) $100 \mathrm{mM} \mathrm{K}^{+}$
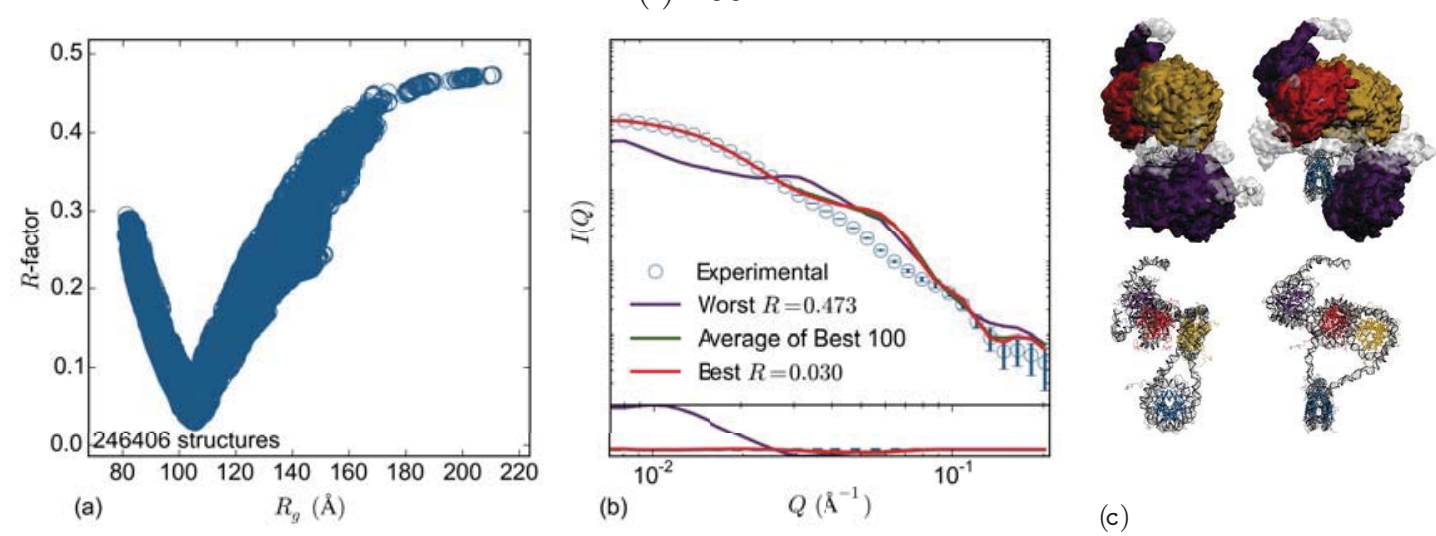

(ii) $1 \mathrm{mM} \mathrm{Mg} \mathrm{Mg}^{2+}$

Figure 60. $4 \times 167$ Sub-Ensembles for the $100 \mathrm{mM} \mathrm{K}^{+}$and $1 \mathrm{mM} \mathrm{Mg}^{2+}$ Data: (a)-(c) as in figs. 58 and 59, namely: (a) $R$-factors versus $R_{g}$ for each structure from the ensemble. (b) experimental $I(Q)$ compared with the $I(Q)$ calculated from the structures with the worst match, the best match, and the average of the 100 best matches; residuals are shown below. (c) two views of the range covered by the best 100 structures (top) with an illustration of the best-matched structures (bottom, animated in the electronic version); the two views are oriented showing the face (left) and side (right) of $\mathrm{NCP}_{2}$. The nucleosome (range) and nucleosome proteins (illustration) are colored red, blue, yellow, and purple respectively for nucleosomes 1 , 2,3 , and 4 . For improved visibility, all structures were aligned using nucleosome 2 (blue) with the nucleosome dyad oriented vertically.

core of the other thereby minimizing the electrostatic forces. The variety in NCP orientations reinforces our observation that the $50 \mathrm{mM} \mathrm{K}{ }^{+}$data showed a transition between the low and high salt measurements, fig. 28,

The relatively low quality in the matches between the higher salt data and model structures makes it difficult to draw any solid conclusions from the resulting sub- 
ensembles. One item to point out is that under maximum ionic screening, the compact structures in $1 \mathrm{mM} \mathrm{Mg}^{2+}$, fig. 60(ii) show much more disorder compared to the array with gH5.

For the $50 \mathrm{mM} \mathrm{K}^{+}, 100 \mathrm{mM} \mathrm{K}^{+}$, and $1 \mathrm{mM} \mathrm{MgCl}_{2}$, models which match the experimental $R_{g}$ and low- $Q$ feature, have an added feature centered around $Q \approx 0.052 \AA^{-1}$ which encodes structural information on the order of $D \approx 120 \AA$. Discussed when comparing the Kratky peaks in the experimental data, at $10 \mathrm{mM} \mathrm{K}^{+}$a similar feature is visible centered at $Q=0.040 \AA^{-1}$, encoding ordering centered at $D=160 \AA$, but this feature diminishes for increased salt. This distance of $160 \AA$ corresponds to the distance between neighboring nucleosomes and we originally contributed the decreased ordering to a less consistent distance between nucleosomes. Examining the models which match the $10 \mathrm{mM} \mathrm{K}^{+}$data, we do see a consistent distance between linked NCPs maintained by straight linker DNA. The model which match the higher salt data show relatively bent linker DNA yielding a decreased distances between adjacent NCPs.

By examining the best-matched model structures, the most likely contributor to the ordering around $\sim 120 \AA$ is the relative distances between $\mathrm{NCP}_{1}-\mathrm{NCP}_{3}$ and $\mathrm{NCP}_{1}-$ $\mathrm{NCP}_{4}$. Using the $R$-factor and a logarithmic $Q$-grid places a strong emphasis on the Guinier region, the best matches having the correct overall size. The $R$ versus $R_{g}$ plots, figs. 59 and 60(a), show a host of structures have the same well matched $R_{g}$ but it still could be that we have not yet explored a wide enough range of configuration space to find single structures which describe the data.

Another possible but less likely source for the ordering seen in the model data, not present in experimental data, could be variations in the overall size of the nucleosome. Initial DNA MC trajectories allowed only the 20 bp fragments of linker DNA to be flexible which indeed maintains a consistent nucleosome structure. Allowing for DNA breathing and DNA unwrapping would introduce significant deviations to structural 
ordering at the nucleosome level, $Q>0.07 \AA^{-1}$, a higher $Q$-range than where the discrepancy occurs. To give consideration to this possibility, additional trajectories simulated up to the first $30 \mathrm{bp}$ wrapping each end of the nucleosome to be flexible, for a total of up to 80 continuous bp between NCP atoms consider as rigid. The energetics of the DNA MC algorithm, particularly the DNA persistence length term of eq. (30), are such that when designated as flexible, the strongly bent DNA wrapping the nucleosome becomes much straighter. This consequently produced structures with a much larger $R_{g}$, and therefore match poorly to the low- $Q$ region of the higher [salt] data. It may be the case that the DNA is more loosely bound in some regions while unwrapping in others but without performing computationally expensive MD simulations, separately exploring the parameters of each individual nucleosome is difficult.

\section{$3 \times 167$ Models}

Select $3 \times 167$ models demonstrated a mix of matching the experimental data well over the entire $Q$-range, 10 and $200 \mathrm{mM} \mathrm{K}^{+}$, and only matching at low and high $Q$-values, 50 and $100 \mathrm{mM} \mathrm{K}^{+}$. The fact that the highest and lowest ion conditions produce reasonable matches indicates that the differentiating factor is not entirely salt dependent, though the full reason remains unclear.

Looking at the different sub-ensembles, for the $10 \mathrm{mM} \mathrm{K}^{+}$data, shown in fig. 61(i), once again we see extended structures with $\mathrm{NCP}_{1}$ and $\mathrm{NCP}_{3}$ significantly separated. In the few structures for which $\mathrm{NCP}_{1}$ approaches $\mathrm{NCP}_{3}$, they arrange in a side-onside orientation. One distinct feature common among the best 100 matched structures is one $\mathrm{NCP}$ is much closer to $\mathrm{NCP}_{2}$ than the other indicating heterogeneous DNA unwrapping.

For models best matched to the 50 and $100 \mathrm{mM} \mathrm{K}^{+}$data, we also see heterogeneous DNA unwrapping. Shown in fig. 61(ii), the $50 \mathrm{mM}$ structures are relatively extended, 

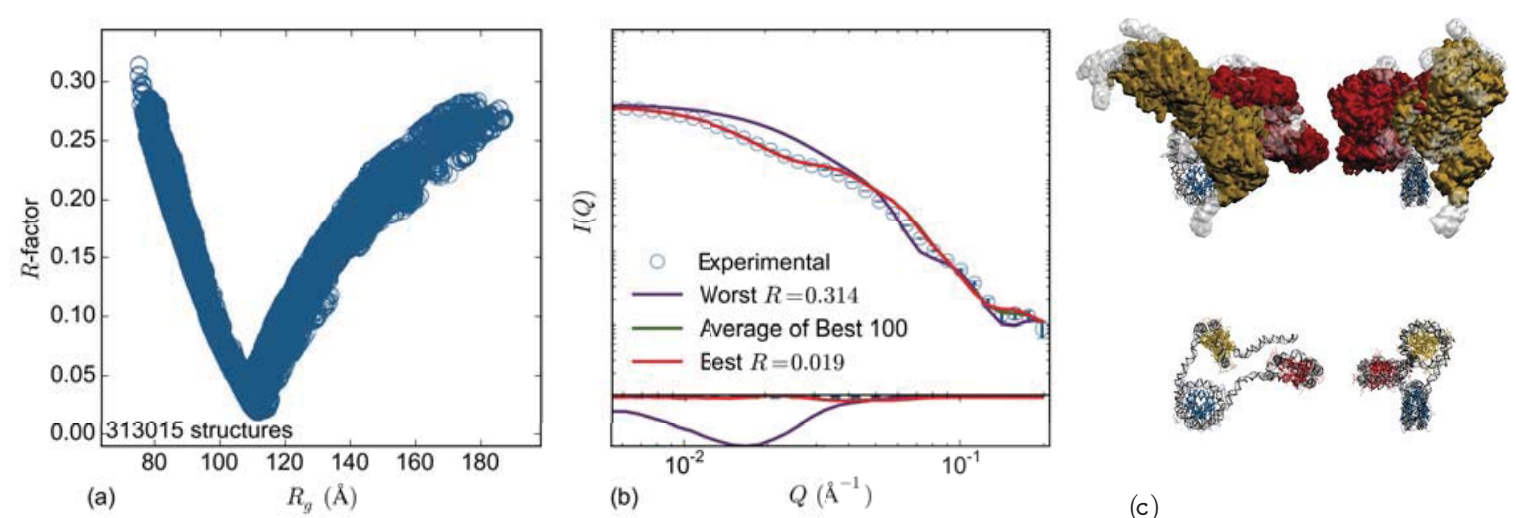

(i) $10 \mathrm{mM} \mathrm{K}^{+}$
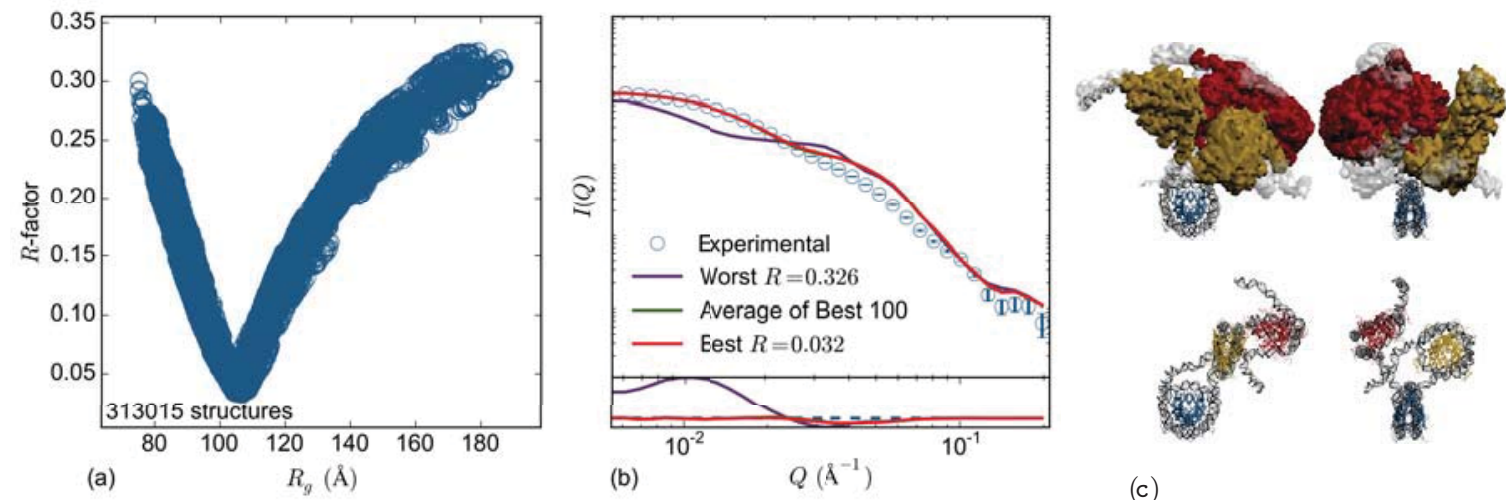

(c)

(ii) $50 \mathrm{mM} \mathrm{K}^{+}$

Figure 61. $3 \times 167$ Sub-Ensembles for the 10 and $50 \mathrm{mM} \mathrm{K} \mathbf{K}^{+}$Data: (a)-(c) as in figs. 58-60, namely: (a) $R$-factors versus $R_{g}$ for each structure from the ensemble. (b) experimental $I(Q)$ compared with the $I(Q)$ calculated from the structures with the worst match, the best match, and the average of the 100 best matches; residuals are shown below. (c) two views of the range covered by the best 100 structures (top) with an illustration of the best-matched structures (bottom, animated in the electronic version); the two views are oriented showing the face (left) and side (right) of $\mathrm{NCP}_{2}$. The nucleosome (range) and nucleosome proteins (illustration) are colored red, blue, yellow, and purple respectively for nucleosomes 1, 2, 3, and 4. For improved visibility, all structures were aligned using nucleosome 2 (blue) with the nucleosome dyad oriented vertically.

but not to the extent seen in the $10 \mathrm{mM}$ models, with $\mathrm{NCP}_{1}$ and $\mathrm{NCP}_{3}$ frequently arranged in a side-on-side or side-on-face orientation. Compared to the $10 \mathrm{mM}$ models, the $100 \mathrm{mM}$ structures, shown in fig. 62(i), are more compact, with $\mathrm{NCP}_{1}$ and $\mathrm{NCP}_{3}$ configured typically in a side-on-face orientation.

The structures that match the $200 \mathrm{mM} \mathrm{K}^{+}$data, shown in fig. 62(ii), are the most 

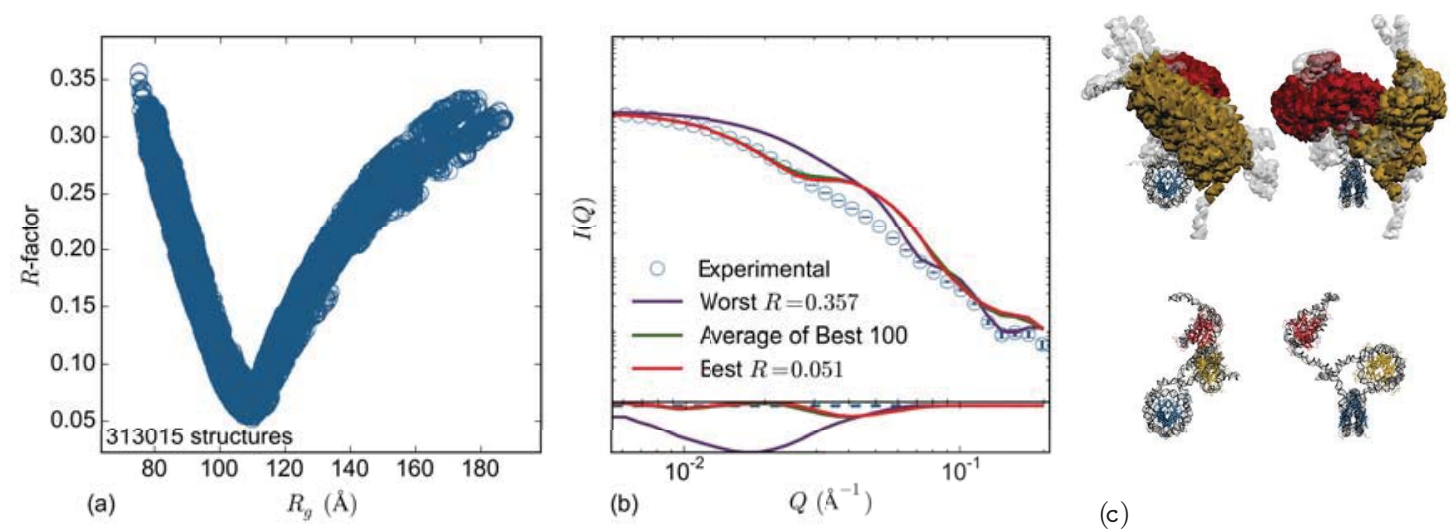

(i) $100 \mathrm{mM} \mathrm{K}^{+}$
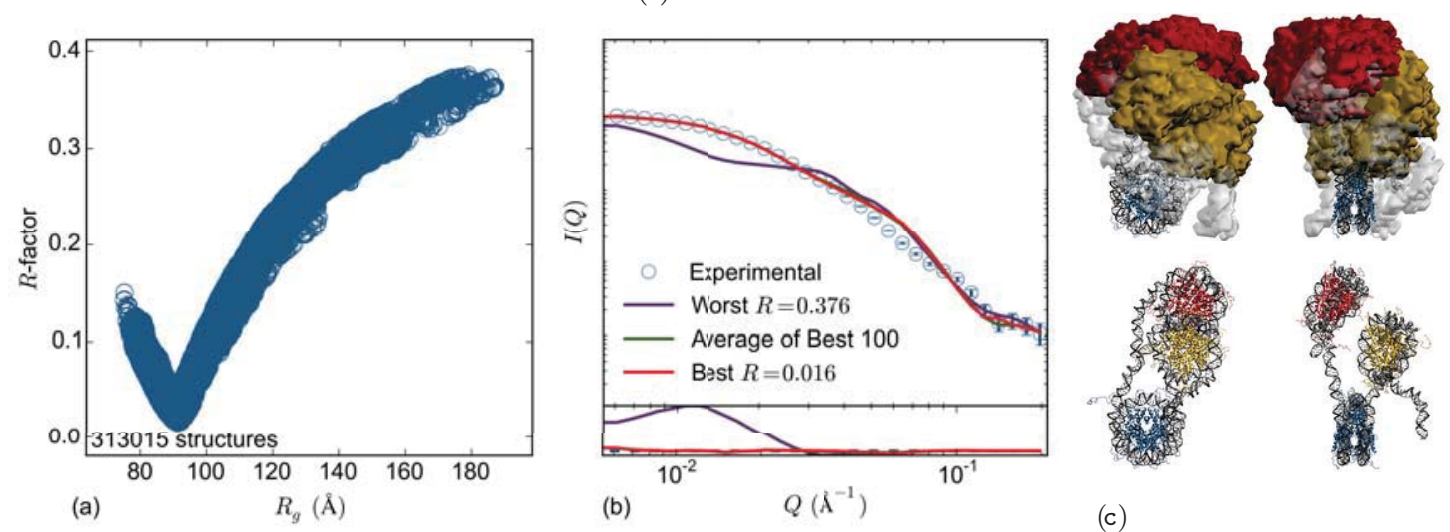

(ii) $200 \mathrm{mM} \mathrm{K}^{+}$

Figure 62. $3 \times 167$ Sub-Ensembles for the 100 and $200 \mathrm{mM} \mathrm{K} \mathbf{K}^{+}$Data: (a)-(c) as in figs. 58-61, namely: (a) $R$-factors versus $R_{g}$ for each structure from the ensemble. (b) experimental $I(Q)$ compared with the $I(Q)$ calculated from the structures with the worst match, the best match, and the average of the 100 best matches; residuals are shown below. (c) two views of the range covered by the best 100 structures (top) with an illustration of the best-matched structures (bottom, animated in the electronic version); the two views are oriented showing the face (left) and side (right) of $\mathrm{NCP}_{2}$. The nucleosome (range) and nucleosome proteins (illustration) are colored red, blue, yellow, and purple respectively for nucleosomes 1, 2, 3, and 4. For improved visibility, all structures were aligned using nucleosome 2 (blue) with the nucleosome dyad oriented vertically.

compact structures. The most common $\mathrm{NCP}$ arrangement has $\mathrm{NCP}_{1}-\mathrm{NCP}_{3}$ in a relative face-on-face packing, resembling that seen in the 1ZBB structure. The other prevalent configuration has $\mathrm{NCP}_{3}$ positioned between $\mathrm{NCP}_{1}$ and $\mathrm{NCP}_{2}$.

For the 50 and $100 \mathrm{mM} \mathrm{K}{ }^{+}$sub-ensembles, the theoretical $I(Q)$ s matches the experimental $I(Q)$ over the low $Q$-region best have a more prominent features centered 
at $\sim 0.049 \AA^{-1}$, encoding ordering in the range of $\sim 130 \AA$. Measuring distances in the best hundred models, $130 \AA$ corresponds to the distances between $\mathrm{NCP}_{1}-\mathrm{NCP}_{3}$ and $\mathrm{NCP}_{2}-\mathrm{NCP}_{3}$ (in the models, $\mathrm{NCP}_{1}$ typically shows more DNA unwrapping which makes the $\mathrm{NCP}_{1}-\mathrm{NCP}_{2}$ distance larger, $\sim 190 \AA$ ). It seems as if the solution structure has the same level of compaction as the models shown, but with a polydisperse distribution of distances between NCPs. Without further information, it is difficult to clearly identify the best course to resolve this discrepancy.

\section{$2 \times 167$ Models}

As for the other constructs measured in $10 \mathrm{mM} \mathrm{K}^{+}$, select structures match the $2 \times 167$ array data over the entire $Q$-range. Compared to the arrangement of $\mathrm{NCP}_{1}$ and $\mathrm{NCP}_{2}$ in the 1ZBB model, these best-matched models show significant DNA unwrapping from the first NCP. The most striking quality of the best-matched structures for this data is the amount that the linker DNA is bent.

DNA in such low salt solutions has a persistence length of $\sim 530 \AA$, but nucleosomes connected by 20 bp of straight linker DNA have a center-to-center distance of $\sim 160 \AA$. In a nucleosome, specific interactions with the histone core proteins give the DNA a radius of curvature of $\sim 50 \AA$, but linker DNA is typically considered as extremely rigid. Recognizing that the scattering process is not unique, i.e., that multiple atomic configurations can lead to the same scattering profile, it may indeed be the case that the linker DNA is more straight, but that nucleosomes are unwrapped and arranged roughly in the positions shown in fig. 63(c). Regardless, it is clear the model structures shown approximate the structure well.

\section{Potential Sources for Complications in Modeling Data}

Our discussion thus far has treated all experimental data as accurately representing the single-particle form-factor. We considered the inability to match structures 
to some of the data, particularly in the region $0.03 \AA^{-1}<Q<0.8 \AA^{-1}$, from the perspectives that we have not sufficiently sampled the possible configuration space or that there are some dynamics in solution that we have not incorporated into the modeling algorithm.

Alternatively, it may be the case that some of the data do not represent the singleparticle form-factor. It may be the case that particles in solution exhibit a significant amount of shape polydispersity, i.e., substantial diversity in particle shape. Such diversity may not just exist among the scattering particles at a given moment but could also originate from the dynamic structure of each individual particle during the measurement exposure time. We mentioned how $\beta(Q)$, in eq. (4), depends on the level of polydispersity and spherical symmetry of the scattering particles. From the example structures shown in figs. 59 and $60(\mathrm{c})$, we see a great deal of shape variations. It is also clear that the structures are not well approximated by a sphere. The amount
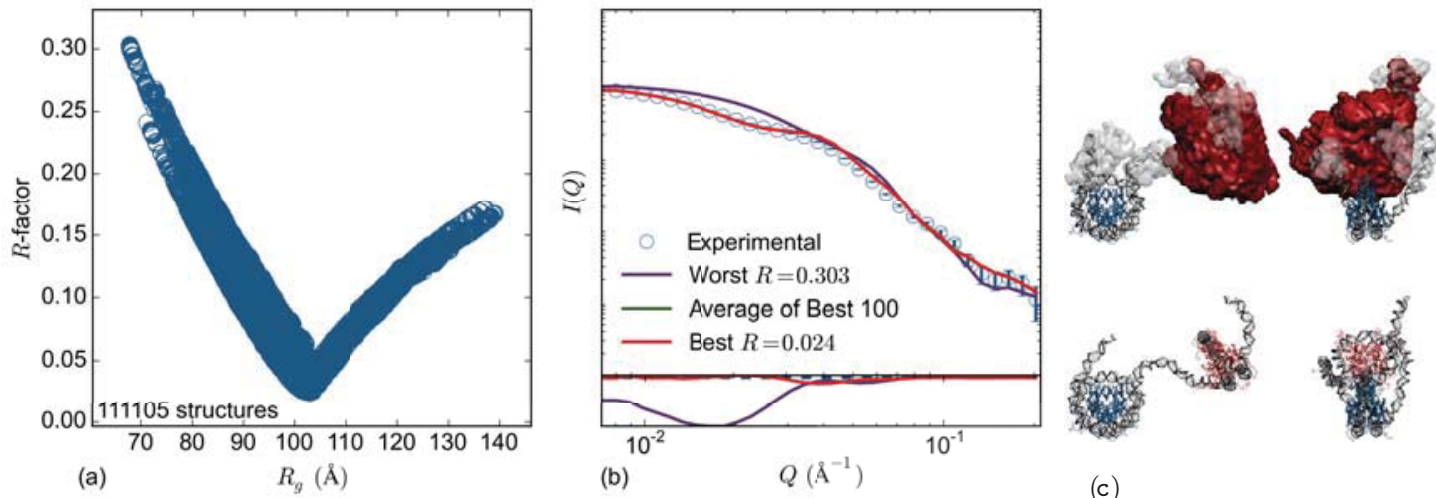

(c)

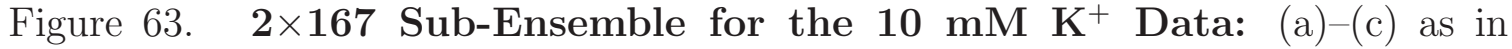
figs. 58-62, namely: (a) $R$-factors versus $R_{g}$ for each structure from the ensemble. (b) experimental $I(Q)$ compared with the $I(Q)$ calculated from the structures with the worst match, the best match, and the average of the 100 best matches; residuals are shown below. (c) two views of the range covered by the best 100 structures (top) with an illustration of the best-matched structures (bottom, animated in the electronic version); the two views are oriented showing the face (left) and side (right) of $\mathrm{NCP}_{2}$. The nucleosome (range) and nucleosome proteins (illustration) are colored red, blue, yellow, and purple respectively for nucleosomes 1, 2, 3, and 4. For improved visibility, all structures were aligned using nucleosome 2 (blue) with the nucleosome dyad oriented vertically. 
of polydispersity and globular nature of the structures could result in the effective structure factor still being significant despite measuring at the lowest possible sample concentrations.

We also address the possibility of interparticle association, or small amounts of aggregation, in the samples. As the $I(0)$ provides a mechanism to determine the molecular weight of the scattering particle, when we scale I(0) by the sample concentration we expect the result to match independent of salt or sample concentrations. Figure 64

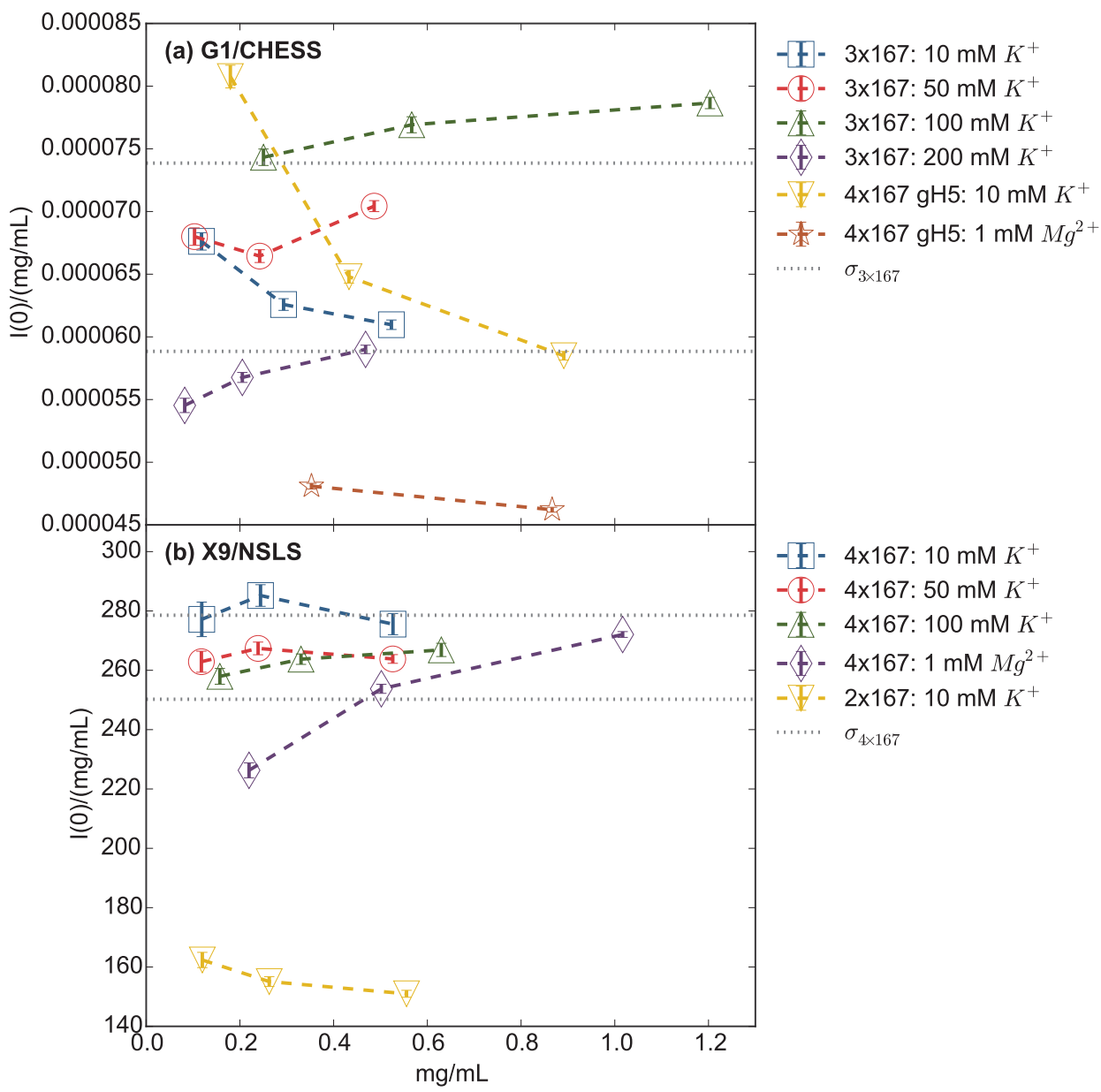

Figure 64. Comparison of $\boldsymbol{I}(\mathbf{0}) /[\mathbf{m g} / \mathbf{m L}]$ : As $I(0)$ depends on the molecular weight, normalizing $\mathrm{I}(0)$ by the mass concentration should produce a consistent quantity independent of mass and salt concentrations. The values are separated depending on if they were measured at G1/CHESS, (a), or X9/NSLS, (b). The dotted horizontal lines represent one standard deviation from the mean value for the $3 \times 167$ samples in (a), and the $4 \times 167$ samples in (b). 
shows the resulting quantities, (a) showing the samples measured at G1/CHESS and (b) showing the samples measured at X9/NSLS. To serve as a point of reference, we show the standard deviation calculated from the 12 measurements of $3 \times 167$ arrays, (a), and the $4 \times 167$ arrays, (b). As for all experimental measurements, we expect variations in the result. We therefore compare values to the standard deviation to look for samples that are consistently higher than the mean, denoting increased molecular weight.

Of the $3 \times 167$ arrays, the 50 and $100 \mathrm{mM} \mathrm{K}^{+}$data were most poorly matched by the model structures. In the $\mathrm{I}(0) /[\mathrm{mg} / \mathrm{mL}]$ comparison, fig. 64(a), every measurement from the $100 \mathrm{mM} \mathrm{K}^{+}$data is outside one standard deviation from the mean. This is not the case for the $50 \mathrm{mM} \mathrm{K}^{+}$data which closely matches the mean. Based on this, it seems more likely that the $100 \mathrm{mM} \mathrm{K}^{+}$data, but not the $50 \mathrm{mM} \mathrm{K}^{+}$data, show signs of association thereby increasing the molecular weight as seen in the $\mathrm{I}(0)$

The distribution of the $\mathrm{I}(0) /[\mathrm{mg} / \mathrm{mL}]$ values for the $4 \times 167$ arrays has one large outlier which results in a standard deviation that contains almost all the data points. Looking for values consistently higher irrespective of the standard deviation, we see that of the different salt concentrations, the $10 \mathrm{mM} \mathrm{K}^{+}$has the highest values. As select models matched this data well, this does not provide strong evidence that association prevented better fits in the higher salt measurements.

Searching for additional possibilities, we compare $I(0)$ and $R_{g}$ values calculated from $P(r)$ to these same values calculated using the Guinier fits. As $P(r)$ is calculated from the entire scattering curve, extracting $I(0)$ from $P(r)$ can be more reliable than that calculated from the Guinier fit [141]. $I(0)$ can be directly calculated from $P(r)$ as,

$$
I(0)=4 \pi \int_{0}^{D_{\max }} P(r) d r .
$$


Table 5. $\quad \boldsymbol{R}_{\boldsymbol{g}}$ and $\boldsymbol{I}(\mathbf{0})$ Calculated from the Guinier Fit Versus the $P(r)$ : For the $R_{g}$, we show both the values and the ratio between the two for easier comparison. For the $I(0)$, we only show the ratio between the two values as they are on a relative scale, and are therefore significantly different depending on where the data was measured. For both ratios, a value of 1.00 indicates a perfect match. For the $P(r)$ values, $R_{g}$ and I(0) were calculated using GNOM [107].

\begin{tabular}{|ll|c|c|c|c|}
\hline \multirow{2}{*}{ Construct } & {$[$ Salt $]$} & $R_{g P(r)}$ & $R_{g I(Q)}$ & $\frac{\mathrm{R}_{g P(r)}}{\mathrm{R}_{g I(Q)}}$ & $\frac{I_{P(r)}(0)}{I_{I(Q)}(0)}$ \\
\hline $2 \times 167$ & $10 \mathrm{mM} \mathrm{K}^{+}$ & $108.0 \pm 1.1 \AA$ & $101.8 \pm 1.8 \AA$ & 1.06 & 1.02 \\
\hline $3 \times 167$ & $10 \mathrm{mM} \mathrm{K}^{+}$ & $116.3 \pm 0.7 \AA$ & $111.7 \pm 1.6 \AA$ & 1.04 & 1.01 \\
& $50 \mathrm{mM} \mathrm{K}^{+}$ & $107.4 \pm 0.5 \AA$ & $106.9 \pm 1.6 \AA$ & 1.00 & 0.99 \\
& $100 \mathrm{mM} \mathrm{K}^{+}$ & $108.8 \pm 0.5 \AA$ & $112.3 \pm 1.5 \AA$ & 0.97 & 0.96 \\
& $200 \mathrm{mM} \mathrm{K}^{+}$ & $93.1 \pm 0.5 \AA$ & $91.6 \pm 1.1 \AA$ & 1.02 & 0.99 \\
\hline $4 \times 167$ & $10 \mathrm{mM} \mathrm{K}^{+}$ & $139.2 \pm 1.6 \AA$ & $133.2 \pm 3.0 \AA$ & 1.05 & 1.02 \\
& $50 \mathrm{mM} \mathrm{K}^{+}$ & $118.9 \pm 0.5 \AA$ & $121.3 \pm 1.8 \AA$ & 0.98 & 0.95 \\
& $100 \mathrm{mM} \mathrm{K}^{+}$ & $129.0 \pm 1.2 \AA$ & $122.4 \pm 1.9 \AA$ & 0.92 & 0.99 \\
& $1 \mathrm{mM} \mathrm{Mg}^{2+}$ & $107.3 \pm 0.4 \AA$ & $105.9 \pm 1.2 \AA$ & 1.01 & 0.99 \\
\hline $4 \times 167 \mathrm{gH} 5$ & $10 \mathrm{mM} \mathrm{K}^{+}$ & $132.0 \pm 0.5 \AA$ & $131.1 \pm 2.0 \AA$ & 1.01 & 0.97 \\
& $1 \mathrm{mM} \mathrm{Mg}^{2+}$ & $83.4 \pm 0.3 \AA$ & $82.2 \pm 0.9 \AA$ & 1.01 & 1.00 \\
\hline
\end{tabular}

Table 5 shows the ratio of $R_{g}$ and $\mathrm{I}(0)$ calculated from the $P(r)$ and calculated using the Guinier fits, $I_{P(r)}(0) / I_{I(Q)}(0)$. Disagreements typically indicate small amounts of aggregation or heterogeneity that primarily affect the low-Q data, and therefore impact the $R_{g}$ calculation [141]. Of the different data sets, the $3 \times 167$ array in $100 \mathrm{mM}$ $\mathrm{K}^{+}$and the $4 \times 167$ array in 50 and $100 \mathrm{mM} \mathrm{K}^{+}$show the largest deviations. These are all among the data which could not be represented by single models from the structure ensembles, but this does not fully account for the match difficulties. The $4 \times 167$ arrays in $1 \mathrm{mM} \mathrm{Mg}^{2+}$ produced much worse fits compared to the $50 \mathrm{mM} \mathrm{K}^{+}$, but the $R_{g}$ and $\mathrm{I}(0)$ from Guinier and $P(r)$ analysis from the $1 \mathrm{mM} \mathrm{Mg}^{2+}$ agree better than those from the $50 \mathrm{mM} \mathrm{K}^{+}$.

Based on the comparisons of $\mathrm{I}(0)$ versus sample concentration and also the $R_{g}$ and $\mathrm{I}(0)$ from Guinier versus $P(r)$ analysis, there is strong evidence that the $3 \times 167$ array 
in $100 \mathrm{mM} \mathrm{K}^{+}$data shows signs of association, and possibly also the $4 \times 167$ array in 50 and $100 \mathrm{mM} \mathrm{K}^{+}$. We are not able to fully reconcile the inability to match the $4 \times 167$ arrays in high salts as results were not conclusive though it may be possible to acquire improved data through more rigorous sample purification. We did attempt filtering the model structures using the back-transformed $P(r), I^{\prime}(Q)=\mathcal{F}^{-1}(\mathcal{F}(I(Q)))$, but results did not show a significant improvement in the match quality.

An important observation from the differences in data quality between the $4 \times 167$ arrays with and without gH5 linker is that the addition of linker histone stabilizes the array, especially in higher salt. Though the data here only provide minimal evidence, it was our experience from repeatedly measuring $4 \times 167$ arrays that those with linker histones produced more reliable measurements.

\section{$4 \times 167$ gH5 Array Structure Properties}

To gain added information from the best fit models of the $4 \times 167$ gH5 array, we further analyzed the scattering and structural features of the best sub-ensemble. For this deeper analysis, we expanded the sub-ensemble from the best 100 to best the 1000 models. Figure 65 shows the calculated scattering from these sub-ensembles. In addition to the best-matched structure, we see that the average of all 1000 structures, and even the worst of the 1000 structures, compares favorably with the experimental data.

To obtain quantified details on the structures in these sub-ensembles, we extracted the relative NCP positions and orientations from the individual structures. Some of these extracted values are the same as those sampled using the rigid body moves in Chapter 3. Tables 6 and 7 compare the same values extracted from the 1ZBB [2] configuration with the mean and standard deviation of those extracted from the $10 \mathrm{mM} \mathrm{K}^{+}$and $1 \mathrm{mM} \mathrm{Mg}^{2+}$ sub-ensembles. For each of these values, we report the results for individual NCP pairs and also an overall average. Table 6 shows the values 


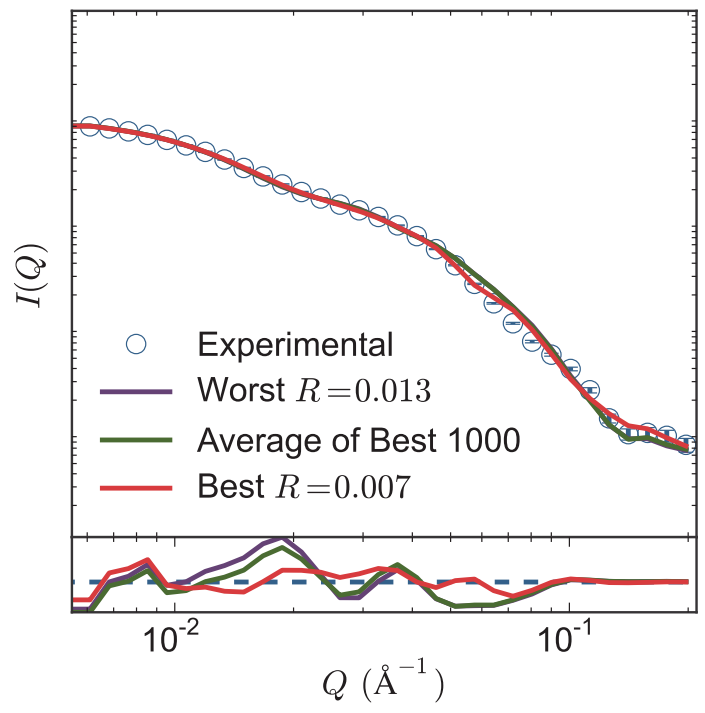

(i) $10 \mathrm{mM} \mathrm{K}^{+}$

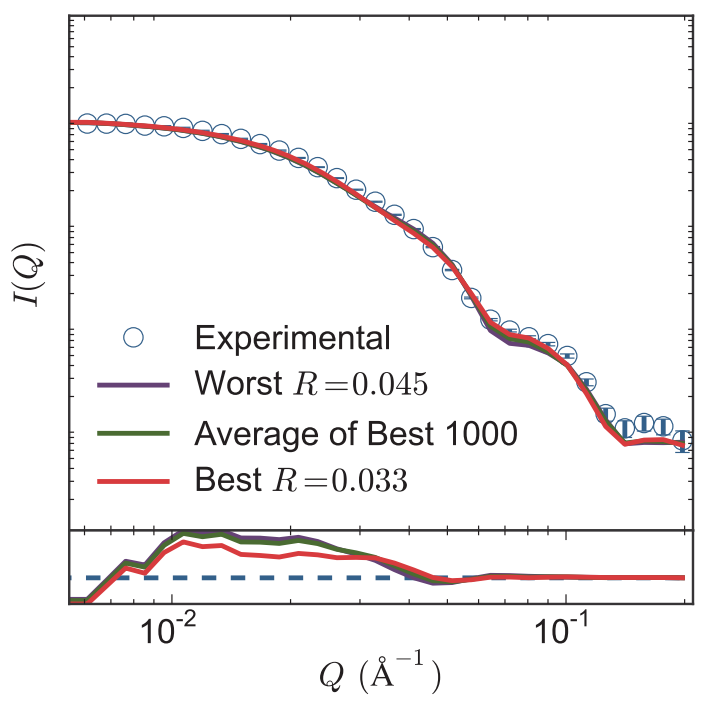

(ii) $1 \mathrm{mM} \mathrm{Mg}^{2+}$

Figure 65. $4 \times \mathbf{1 6 7}$ gH5 Sub-ensembles of the Best 1000 Models: The experimental $I(Q)$ for $4 \times 167 \mathrm{gH} 5$ in $10 \mathrm{mM} \mathrm{K}^{+}$, (i), and $1 \mathrm{mM} \mathrm{Mg}^{2+}$, (ii), compared with the best 1000 structures from the complete ensemble.

from adjacent $\mathrm{NCPs}$, e.g., $\mathrm{NCP}_{1}-\mathrm{NCP}_{2}$. Table 7 shows the values from next-nearestneighbor NCPs, referred to as stacked NCPs, e.g., $\mathrm{NCP}_{1}-\mathrm{NCP}_{3} \cdot{ }^{17}$

The quantities of interest are the stack distance, the separation distance, the opening angle, the angle between cylinder axes, and the twist angle. Though some of these values were introduced in Chapter 3, we review the definition of each quantity for clarity. The stack and separation distances are respectively the center-to-center distances between stacked NCPs and adjacent NCPs. For stacked NCPs, this distance correlates with the opening angle, which is the angle between the segments connecting three successive NCPs. The angle between cylinder axes describes the alignment of stacked NCPs and is calculated using the axis passing through the center of a cylinder fit to the $\mathrm{C}^{\prime}$ atoms of each NCP. The twist angle is the dihedral angle between the cylinder axes of adjacent NCPs; more explicitly, it is the angle between the planes

\footnotetext{
17 We refer to next-nearest-neighbor NCPs as stacked NCPs because they are stacked in the 1ZBB configuration. This is not always the case for the structures in the sub-ensembles, particularly for the $10 \mathrm{mM} \mathrm{K}^{+}$sub-ensemble.
} 
defined by the two cylinder axes and the center-to-center segment between the NCPs. We define a positive dihedral angle using the right-hand rule along the line connecting $\mathrm{NCP}_{i}$ to $\mathrm{NCP}_{i+1}[150]$. The difference between the angle between cylinder axes and the twist angle is subtle and therefore can lead to confusion. The angle between cylinder axes is the minimum angle between the two axes and can be obtained by placing two vectors at the same origin, each vector oriented parallel to one of the cylinder axes, then calculating the angle between the vectors. The twist angle differs as it is the angle which rotates the two axes about the line connecting their origins bringing them to the same plane but not necessarily parallel to each other. We report the twist angle for adjacent NCPs and the angle between cylinder axes for the stacked NCPs as these provide the most useful information regarding their relative orientations; additionally, the linker DNA approximates the center-to-center segment connecting adjacent NCPs but there is no such link between stacked NCPs.

Table 6. Stacked NCP Features from the Best $10004 \times 167$ gH5 Models: The relative nucleosome positions and orientations for stacked NCPs, e.g., $\mathrm{NCP}_{1}-\mathrm{NCP}_{3}$. Values are displayed as the mean plus or minus one standard deviation. Ensemble results are compared to the $1 \mathrm{ZBB}$ configuration [2] to demonstrate the differences between the crystal and solution structures.

\begin{tabular}{|ll|c|l|l|}
\cline { 3 - 5 } \multicolumn{1}{l|}{} & \multicolumn{1}{c|}{$\begin{array}{c}\text { Stack } \\
\text { Distance }\end{array}$} & $\begin{array}{c}\text { Opening } \\
\text { Angle }\end{array}$ & $\begin{array}{l}\text { Angle Between } \\
\text { Cylinder Axes }\end{array}$ \\
\hline $10 \mathrm{mM} \mathrm{K}^{+}$ & $\mathrm{NCP}_{1}-\mathrm{NCP}_{3}$ & $95.2 \pm 2.5 \AA$ & $35.0^{\circ} \pm 1.2^{\circ}$ & $10.9^{\circ} \pm 3.9^{\circ}$ \\
& $\mathrm{NCP}_{2}-\mathrm{NCP}_{4}$ & $247.3 \pm 12.9 \AA$ & $82.9^{\circ} \pm 7.9^{\circ}$ & $38.5^{\circ} \pm 23.5^{\circ}$ \\
& $\mathrm{NCP}_{i}-\mathrm{NCP}_{i+2}$ & $171.2 \pm 76.6 \AA$ & $58.9^{\circ} \pm 24.6^{\circ}$ & $24.7^{\circ} \pm 21.8^{\circ}$ \\
\hline $1 \mathrm{mM} \mathrm{Mg}^{2+}$ & $\mathrm{NCP}_{1}-\mathrm{NCP}_{3}$ & $67.6 \pm 3.5 \AA$ & $27.9^{\circ} \pm 1.7^{\circ}$ & $22.5^{\circ} \pm 5.3^{\circ}$ \\
& $\mathrm{NCP}_{2}-\mathrm{NCP}_{4}$ & $64.1 \pm 4.0 \AA$ & $26.0^{\circ} \pm 2.1^{\circ}$ & $20.3^{\circ} \pm 3.3^{\circ}$ \\
& $\mathrm{NCP}_{i}-\mathrm{NCP}_{i+2}$ & $65.9 \pm 4.1 \AA$ & $26.9^{\circ} \pm 2.1^{\circ}$ & $21.4^{\circ} \pm 4.6^{\circ}$ \\
\hline $12 \mathrm{BB}$ & $\mathrm{NCP}_{1}-\mathrm{NCP}_{3}$ & $57.4 \AA$ & $22.7^{\circ}$ & $11.2^{\circ}$ \\
\hline \multirow{2}{*}{ Configuration } & $\mathrm{NCP}_{2}-\mathrm{NCP}_{4}$ & $57.5 \AA$ & $22.8^{\circ}$ & $11.6^{\circ}$ \\
& $\mathrm{NCP}_{i}-\mathrm{NCP}_{i+2}$ & $57.4 \pm 0.1 \AA$ & $22.8^{\circ} \pm 0.0^{\circ}$ & $11.4^{\circ} \pm 0.2^{\circ}$ \\
\hline
\end{tabular}




\section{Models of $4 \times 167 \mathrm{gH} 5$ in $10 \mathrm{mM} \mathrm{\textrm {K } ^ { + }}$}

The $10 \mathrm{mM} \mathrm{K}^{+}$models show significant but asymmetric deviations from the 1ZBB configuration. These differences are apparent in both the adjacent NCP results, in table 6 , and the stacked NCP results, in table 7 . The most prominent differences is a much larger distance between $\mathrm{NCPs}$, especially $\mathrm{NCP}_{4}$, and that the three relatively compact NCPs are less twisted.

Compared to the 1ZBB configuration, models which match the experimental data best are considerably less compact and have one NCP significantly extended. The distance between stacked NCPs in the $10 \mathrm{mM} \mathrm{K}^{+}$models is nearly twice as large for $\mathrm{NCP}_{1}-\mathrm{NCP}_{3}$ and five times as large for $\mathrm{NCP}_{2}-\mathrm{NCP}_{4}$. We also point out that the $\mathrm{NCP}_{3}-\mathrm{NCP}_{4}$ distance is notably larger than the other $\mathrm{NCP}_{i}-\mathrm{NCP}_{i+1}$ distances, indicating DNA unwrapping from $\mathrm{NCP}_{4}$. We recognize that construct symmetry together with stochastic MC sampling would naturally lead to an equal sampling of $\mathrm{NCP}_{1}$ and $\mathrm{NCP}_{4}$ extended. We do not see this symmetric sampling of both extended states because of the manner in which we performed MC sampling. To investigate various levels of extended structures, we generated structures allowing for movements of only $\mathrm{NCP}_{4}$ and also movements of either $\mathrm{NCP}_{3}$ or $\mathrm{NCP}_{4}$. Though unlikely, it could provide added information to additionally sample structures with $\mathrm{NCP}_{1}$ extended or $\mathrm{NCP}_{1}$ and $\mathrm{NCP}_{2}$. In this case, rather than grouping according to NCP number, it would make more sense to group the stack distances by their magnitude, i.e., grouping the extended $\mathrm{NCP}_{i}-\mathrm{NCP}_{i+2}$ versus the compact $\mathrm{NCP}_{i}-\mathrm{NCP}_{i+2}$.

The other noticeable difference from the 1ZBB configuration is that the three relatively compact NCPs show a marked change in twist between adjacent NCPs. Where this angle is $-92.4^{\circ}$ for the $1 \mathrm{ZBB}$ structure, the average twist angle for $\mathrm{NCP}_{1}-\mathrm{NCP}_{2}$ and $\mathrm{NCP}_{2}-\mathrm{NCP}_{3}$ is only $-35.25^{\circ}$. The $-92.4^{\circ}$ twist in the $1 \mathrm{ZBB}$ model structure forces the linker DNA strands to cross as they extend to the adjacent nucleosomes. This is not the case for the $10 \mathrm{mM} \mathrm{K} \mathrm{K}^{+}$models. We see in panel (c) of fig. 58(i), that 
these linker DNA fragments straighten allowing the NCPs to spread apart while also changing the reducing the magnitude of twist between NCPs. This happens simultaneously for stacked NCPs as the relative orientation between $\mathrm{NCP}_{1}-\mathrm{NCP}_{3}$ shows essentially no charge.

Table 7. Adjacent NCP Features from the Best $10004 \times 167$ gH5 Models: The relative nucleosome positions and orientations for adjacent $\mathrm{NCPs}$, e.g., $\mathrm{NCP}_{1-}$ $\mathrm{NCP}_{2}$. Values are displayed as the mean plus or minus one standard deviation. The twist angle represents the clockwise rotation of $\mathrm{NCP}_{i}$ about the center-to-center line $\mathrm{NCP}_{i}-\mathrm{NCP}_{i+1}$ that brings the cylinder axes of the two NCPs to the same plane. Ensemble results are compared to the $1 \mathrm{ZBB}$ configuration [2] to demonstrate the differences between the crystal and solution structures.

\begin{tabular}{|ll|l|c|}
\cline { 3 - 4 } \multicolumn{1}{c|}{} & \multicolumn{1}{c|}{$\begin{array}{c}\text { Separation } \\
\text { Distance }\end{array}$} & \multicolumn{1}{c|}{$\begin{array}{c}\text { Twist } \\
\text { Angle }\end{array}$} \\
\hline $10 \mathrm{mM} \mathrm{K}^{+}$ & $\mathrm{NCP}_{1}-\mathrm{NCP}_{2}$ & $150.8 \pm 4.4 \AA$ & $26.8^{\circ} \pm 6.2^{\circ}$ \\
& $\mathrm{NCP}_{2}-\mathrm{NCP}_{3}$ & $162.9 \pm 3.4 \AA$ & $43.7^{\circ} \pm 6.2^{\circ}$ \\
& $\mathrm{NCP}_{3}-\mathrm{NCP}_{4}$ & $208.4 \pm 9.6 \AA$ & $76.8^{\circ} \pm 65.9^{\circ}$ \\
& $\mathrm{NCP}_{i}-\mathrm{NCP}_{i+1}$ & $174.0 \pm 25.6 \AA$ & $49.1^{\circ} \pm 43.6^{\circ}$ \\
\hline $1 \mathrm{mM} \mathrm{Mg}^{2+}$ & $\mathrm{NCP}_{1}-\mathrm{NCP}_{2}$ & $143.0 \pm 2.2 \AA$ & $90.3^{\circ} \pm 3.9^{\circ}$ \\
& $\mathrm{NCP}_{2}-\mathrm{NCP}_{3}$ & $136.3 \pm 1.5 \AA$ & $100.2^{\circ} \pm 4.9^{\circ}$ \\
& $\mathrm{NCP}_{3}-\mathrm{NCP}_{4}$ & $145.9 \pm 3.1 \AA$ & $98.0^{\circ} \pm 4.5^{\circ}$ \\
& $\mathrm{NCP}_{i}-\mathrm{NCP}_{i+1}$ & $141.7 \pm 4.7 \AA$ & $96.2^{\circ} \pm 6.1^{\circ}$ \\
\hline $12 B B$ & $\mathrm{NCP}_{1}-\mathrm{NCP}_{2}$ & $147.6 \AA$ & $91.7^{\circ}$ \\
Configuration & $\mathrm{NCP}_{2}-\mathrm{NCP}_{3}$ & $142.4 \AA$ & $93.0^{\circ}$ \\
& $\mathrm{NCP}_{3}-\mathrm{NCP}_{4}$ & $147.5 \AA$ & $92.4^{\circ}$ \\
& $\mathrm{NCP}_{i}-\mathrm{NCP}_{i+1}$ & $145.8 \pm 2.5 \AA$ & $92.4^{\circ} \pm 0.5^{\circ}$ \\
\hline
\end{tabular}

\section{Models of $4 \times 167 \mathrm{gH} 5$ in $1 \mathrm{mM} \mathrm{Mg}^{2+}$}

Compared to the $10 \mathrm{mM} \mathrm{K}{ }^{+}$models, the $1 \mathrm{mM} \mathrm{Mg}^{2+}$ models deviate more symmetrically from the $1 \mathrm{ZBB}$ configuration. This symmetry in deviations is shown by a consistency in the inter-NCP distances and angles, though the standard deviations indicate a reasonable amount of variations. In general, these charges represent a loosely packed version of the 1ZBB configuration. More specifically, we see increased 

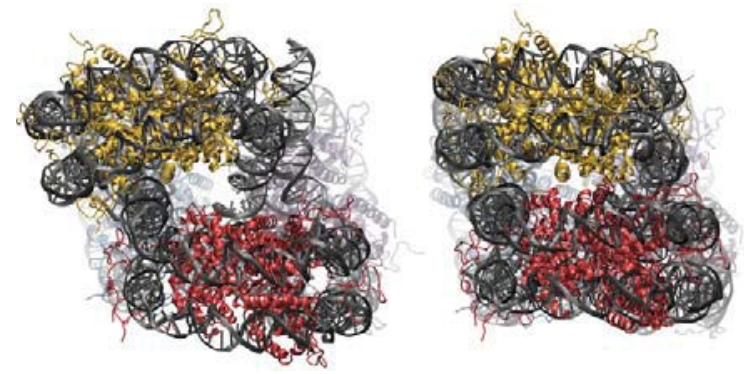

Figure 66. Comparison of a Sample $1 \mathrm{mM} \mathrm{Mg}^{2+} 4 \times 167$ gH5 Model to the 1ZBB Model: The model structures best matched to the $4 \times 167 \mathrm{gH} 5$ in $1 \mathrm{mM}$ $\mathrm{Mg}^{2+}$ data (sample shown on the left) resemble a loose packed version of the 1ZBB crystal structure (right). This sample structure shows a common feature in the 1000 best-matched structures in which the $\mathrm{NCP}_{3}$ is twisting around $\mathrm{NCP}_{1}$.

distance and decreased alignment between stacked NCPs, as well as decreased distance and increased twist between adjacent NCPs.

In reference to the $1 \mathrm{ZBB}$ structure, for the $1 \mathrm{mM} \mathrm{Mg}^{2+}$ models the average distance between stacked NCPs increases by $14.8 \%$ from $57.4 \AA$ to $65.9 \AA$, while the angle between cylinder axes is almost double. The increase in stack distance is a combined result of increasing the opening angle and also face-on-face sliding of stacked NCPs. The increased angle between cylinder axes is also partially caused by an increased opening angle. In general, these differences combined with the sizable variance among the ensemble indicate more disorder in the stacking of NCPs.

A major difference between the increased opening angle in these models compared to the user directed rigid body models is that the NCP separation is combined with a decreased distance between adjacent NCPs. These simultaneous changes maintain a consistent $R_{g}$, which was not the case when we simply increased the opening angle. We note that the decreased distance between adjacent NCPs is much more pronounced between $\mathrm{NCP}_{1}-\mathrm{NCP}_{2}$ and $\mathrm{NCP}_{2}-\mathrm{NCP}_{3}$ compared to $\mathrm{NCP}_{3}-\mathrm{NCP}_{4}$.

In addition to the fairly symmetric changes discussed, adjacent NCPs show an asymmetric change in the twist angle. Compared to the 1ZBB structure, the twist angle between $\mathrm{NCP}_{2}-\mathrm{NCP}_{3}$ and $\mathrm{NCP}_{3}-\mathrm{NCP}_{4}$ increases by $\sim 6^{\circ}$ while the twist of 
$\mathrm{NCP}_{1}-\mathrm{NCP}_{2}$ is essentially unchanged. A consequence of these asymmetric changes is that $\mathrm{NCP}_{3}$ twists around $\mathrm{NCP}_{1}$ approaching a face-on-side orientation, illustrated in fig. 66 .

These commonalities in relative NCPs positions and orientations illuminate the packing of the $4 \times 167 \mathrm{gH} 5$ array in $1 \mathrm{mM} \mathrm{Mg}^{2+}$ solution. By extracting these quantities from the sub-ensemble of best-matched structures, we recognize trends in the individual structures, each of which provide a good description to the experimental data. Comparing this information with the 1ZBB crystal structure provides a valuable extension to understanding to the cellular packing of nucleosome arrays as these solution conditions more closely approximate the cellular environment.

\section{Chapter Summary}

In this chapter we have focused on representing the experimental SAXS data from Chapter 3 using model structures generated with the DNA-MC algorithm described in Chapter 4. Using this algorithm we generated over 240000, 310000, and 110000 model structures of the $4 \times 167,3 \times 167$, and $2 \times 167$ arrays respectively. To decide on a discrepancy metric for filtering these models, we considered the $R$-factor, the weighted $R$-factor, the $F$-factor, and the $\chi^{2}$ statistic. We demonstrated how each metric weights $Q$-regions differently when models cannot be found to match the entire $Q$-region.

Despite $\chi^{2}$ being the most commonly used metric, we chose to rank structures using the $R$-factor as it showed the greatest ability to match the $R_{g}$, a fundamental property of a molecule. Further, our experience showed little difference between the metrics when models could be found that matched over the entire $Q$-range.

From the comparisons of structure ensembles to experimental data, we found well-matched models for a majority of the experimental data. Examining the most favorable models for each data set, we observed NCPs arranged in side-on-face and side-on-side orientations depending on the level of compaction induced by the ionic 
screening. In an effort to identify possible association in the samples, we scrutinized the $\mathrm{I}(0)$ scaled by the concentration and identified the $3 \times 167$ array in $100 \mathrm{mM} \mathrm{K} \mathrm{K}^{+}$ as an outlier. Comparing the $R_{g}$ and $\mathrm{I}(0)$ calculations from Guinier analysis and $P(r)$ integration showed the $3 \times 167$ array in $100 \mathrm{mM} \mathrm{K}^{+}$and the $4 \times 167$ array in 50 and $100 \mathrm{mM} \mathrm{K}^{+}$showed the largest differences. These differences may indicate interparticle association, but it remains unclear as other data of lower quality showed better agreement.

From the sub-ensembles of the 1000 models which best matched the $4 \times 167 \mathrm{gH} 5$ data sets, we showed that of these models, the best, worst, and average of all 1000 showed each represented the experimental data well. In light of this, it is important to recognize that the solution structure of molecules is naturally dynamic, particularly for molecules with relative mobility, such as nucleosome arrays. Moreover, the number of intrinsic constraints in a SAXS measurement is largely unknown, but it is commonly considered to be an underdetermined mathematical problem. An infinite number of solutions can exist for such highly underdetermined problems [140]. Consequently, one should avoid selecting a single structure, or a single linear combination of structures, as a definitive representation of the scattering particle. Instead, the best course is to observe commonalities and look for trends rather than presuming there is a single structure that can fully represent the solution conformation. Increased ensemble refinement can be achieved by further constraining the problems through computational means, such as calculating the free energy of individual structures [127], or experimental means, such as Förester resonance energy transfer (FRET) $[29,151]$.

Taking this into account, we analyzed these sub-ensembles of 1000 structures to extract detailed information regarding the relative position and orientations of NCPs within the $4 \times 167 \mathrm{gH} 5$ array. These results demonstrate the power of using SAXS together with MC modeling to extract specific structural parameters from 
biological molecules in solution. Such structural parameters expand on the static structures obtained through methods such as crystallography and electron microscopy by probing molecules in a more cellular-like environment.

We intend to apply this same analysis to the other structure ensembles, specifically in the cases for which data could be described by model structures over the entire $Q$-range. We will look for trends in the best-matched structures in response to varied ionic conditions and as the number of nucleosome repeats is increased. For data which could not be represented by model structures, we will further consider the source of the discrepancy. Additional measurements may be required but might not produce improved results without the availability of higher flux X-ray beans to measure samples at a sufficiently low sample concentration. We may also perform additional experimental studies to further constrain the structure ensemble. 


\section{Chapter 6: Summary and Outlook}

We set out to determine quantitative information regarding the dynamic conformation of nucleosome arrays in solution using experimental SAXS. Toward this end, we developed a CG simulation algorithm for dsDNA which rapidly generates ensembles of structures through Metropolis MC sampling of a Markov chain. The atomic models generated by this algorithm provide a valuable tool for modeling SAS data but also have a much broader applicability for more general modeling DNA and DNA complexes. By filtering structure ensembles of nucleosome arrays against experimental SAXS profiles, we identified individual structures and sub-ensembles which accurately described the experimental scattering. These favorably matched structures offer the means to extract statistical representations of relative positions and orientations of the nucleosomes within the arrays represented.

For the $4 \times 167$ gH5 arrays, we compared structural parameters to the 1 ZBB crystal structure of a highly similar nucleosome array construct. Through this comparison, we identified specific deviations between the solution structures in $10 \mathrm{mM} \mathrm{K}^{+}$and $1 \mathrm{mM}$ $\mathrm{Mg}^{2+}$ and the crystal structure. The compact structures resulting from high ionic screening, $1 \mathrm{mM} \mathrm{Mg}^{2+}$, strongly resemble the crystallized structure, with the solution structure approximating a loosely packed version of the crystal structure. The most prominent difference between the structure models of $4 \times 167 \mathrm{gH} 5$ in $1 \mathrm{mM} \mathrm{Mg}^{2+}$ and the $1 \mathrm{ZBB}$ model is an average $7.2 \AA$ increase of the center-to-center distance between stacked NCPs, occurring as a result of NCP sliding and an increased opening angle. Recognizing that these model structures lacked the gH5 protein and were therefore not an identical molecular match to the experimental system, we intend to align a model of the gH5 protein to an expanded best-matched ensemble then repeat the scattering calculation and analysis. We also plan to refine this detailed analysis as we investigate the other sub-ensembles which accurately represent experimental results.

Having only recently completed the CG DNA simulation algorithm, we are cur- 
rently in the process of fully integrating it with the full SASSIE package to allow for simultaneous sampling of both DNA and protein conformations. This added modeling capability applies particularly well for modeling DNA unwrapping from the nucleosome core. For the results shown, we separately sampled protein conformation to produce protein configurations that would not allow for DNA unwrapping. In this vein, the combined DNA-protein algorithm shows promise toward generating representative models which describe SAS measurements of DNA unwrapping from single nucleosomes. We are also looking at applying this algorithm to model SAXS measurements of the DNA structure effects of DNA-binding proteins, such as Poly ADP-ribose polymerase more commonly referred to as PARP.

Extending this combination of SAXS measurements with atomic modeling, we also look forward to examining more diverse nucleosome arrays in a more extensive range of sample conditions. Thus far, we have primarily focused on variations to the ionic screening and the addition of linker histones, specifically the globular variants of the $\mathrm{H} 5$ and $\mathrm{H} 1$. In Chapter 1, we discussed several additional factors that affect chromatin structure, including molecular crowding, genetic sequence variation, and epigenetic modifications of the histones including truncation charged tails. To obtain an increased understanding of chromatin structure, we can examine additional ionic conditions, molecular crowding, arrays designed with longer DNA linkers and more NCP repeats, and nucleosomes prepared using gH3 or gH4 proteins as well as lysine acetylation.

A simple extension of our previous studies would be to measure arrays in $\mathrm{Mg}^{2+}$ concentrations similar to the crystallization conditions. Noting this is above the 2-4 mM $\left[\mathrm{Mg}^{2+}\right]$ known to precipitate NCPs [52], the NCPs resuspend as the $\mathrm{Mg}^{2+}$ concentration approaches the $180 \mathrm{mM}$ Schalch et al. used to crystallize the $4 \times 167$ array [2]. Under such strong ionic screening, we would expect to observe loosely packed structures similar to the $4 \times 167 \mathrm{gH} 5$ arrays in $1 \mathrm{mM} \mathrm{Mg}^{2+}$, but from arrays 
without the gH5 linker. This would allow for a detailed comparison to the 1ZBB model, similar to that shown for the $4 \times 167 \mathrm{gH} 5$ arrays in $1 \mathrm{mM} \mathrm{Mg}^{2+}$.

To more closely mimic the cellular environment, it would be instructive to couple variations of ionic conditions to different amounts of molecular crowding. This will require investigation into possible candidates of neutral osmolytes as one of the more commonly used osmolytes, polyethylene glycol, has been shown to associate with protein molecules [152].

In addition to varying the solution conditions, it will be beneficial to extend this research to include arrays with various lengths of linker DNA between NCPs and also increasing the number of NCPs. As a result of the helical nature of DNA, increasing the linker length not only changes the distance between neighboring NCPs but it also changes their relative twist angle. The natural solution conformation of the DNA helix repeats roughly every $10 \mathrm{bp}$ [153] and it is proposed that linker DNA favors quantized lengths of either $10 \mathrm{n}$ or $10 \mathrm{n}+5$ [154]. Based on this, in addition to the arrays with $20 \mathrm{bp}$ linker already measured, we propose to also examine linker lengths of 25 and $30 \mathrm{bp}$. This is likely to not only affect the relative twist between nucleosomes but also the extension at low salt concentrations and the degree of disorder for the compact structures in higher salt concentrations. To get a better representation of chromatin requires we also increase the number of nucleosomes in the array. Studying arrays with more NCPs will be challenging as scattering from large molecules requires accurate data at lower $Q$-values to capture the Guinier region, witnessed by the few points in the $R_{g}$ fits for the $12 \times 167$ arrays. For this reason, the limitations of current SAXS facilities may only allow for measuring larger structure when compacted by high salt concentrations.

For epigenetic modification, it will be most enlightening to focus on acetylation of $\mathrm{H} 4 \mathrm{~K} 16$ and also the truncation of the $\mathrm{H} 3$ and $\mathrm{H} 4$ histone tails. We expect to observe significant structure difference from nucleosomes with H4K16 acetylation as 
this change was shown to de-compact NCP arrays allowing enzymatic access [52-54]. This particular acetylation mark is also significant as it was identified as a switch for changing in vivo chromatin from a repressive state to a transcriptionally active one [51]. We are most interested in studying arrays with globular variants of the H3 and H4 proteins as these are known to be more significant in chromatin compaction [50, 52]. Building on the mononucleosome studies of gH3 and gH4 nucleosomes, it will be interesting to probe how the differences seen in high salt interactions propagate from single nucleosomes to arrays of nucleosomes. 


\section{References}

[1] C. A. Davey, D. F. Sargent, K. Luger, A. W. Maeder, and T. J. Richmond, "Solvent Mediated Interactions in the Structure of the Nucleosome Core Particle at 1.9 A Resolution," Journal of Molecular Biology, vol. 319, no. 5, pp. 1097 1113, Jun. 2002. DOI: 10.1016/S0022-2836(02)00386-8.

[2] T. Schalch, S. Duda, D. F. Sargent, and T. J. Richmond, "X-ray structure of a tetranucleosome and its implications for the chromatin fibre," Nature, vol. 436, no. 7047, pp. 138-141, Jul. 2005. DOI: 10.1038/nature03686.

[3] J. C. Phillips, R. Braun, W. Wang, J. Gumbart, E. Tajkhorshid, E. Villa, C. Chipot, R. D. Skeel, L. Kalé, and K. Schulten, "Scalable molecular dynamics with NAMD," Journal of Computational Chemistry, vol. 26, no. 16, pp. 17811802, Dec. 2005. DOI: 10.1002/jcc. 20289.

[4] P. T. Lowary and J. Widom, "New DNA sequence rules for high affinity binding to histone octamer and sequence-directed nucleosome positioning," Journal of molecular biology, vol. 276, no. 1, pp. 19-42, 1998. DOI: 10.1006/jmbi.1997. 1494.

[5] T. Schlick, J. Hayes, and S. Grigoryev, "Toward Convergence of Experimental Studies and Theoretical Modeling of the Chromatin Fiber," Journal of Biological Chemistry, vol. 287, no. 8, pp. 5183-5191, Feb. 2012. DOI: 10.1074 / jbc.R111.305763.

[6] G. Li and D. Reinberg, "Chromatin higher-order structures and gene regulation," Current Opinion in Genetics \& Development, Chromosomes and expression mechanisms, vol. 21, no. 2, pp. 175-186, Apr. 2011. DOI: 10.1016/j . gde. 2011.01 .022 
[7] A. T. Annuziato, "DNA Packaging: Nucleosomes and Chromatin," Nature Education, 1(1):26, 2008.

[8] K. E. van Holde, "Chromatin Structure and Transcription," in Chromatin, ser. Springer Series in Molecular Biology, Springer New York, 1989, pp. 355-408.

[9] E. I. Campos and D. Reinberg, "Histones: Annotating Chromatin," Annual Review of Genetics, vol. 43, no. 1, pp. 559-599, Dec. 2009. DOI: 10.1146/ annurev.genet.032608.103928.

[10] J. Widom, "Chromosome structure and gene regulation," Physica A: Statistical Mechanics and its Applications, vol. 244, no. 1-4, pp. 497-509, Oct. 1997. DOI: 10.1016/S0378-4371(97)00225-2.

[11] H. G. Garcia, P. Grayson, L. Han, M. Inamdar, J. Kondev, P. C. Nelson, R. Phillips, J. Widom, and P. A. Wiggins, "Biological consequences of tightly bent DNA: The other life of a macromolecular celebrity," Biopolymers, vol. 85, no. 2, pp. 115-130, Feb. 2007. DOI: 10.1002/bip. 20627.

[12] D. M. J. Duhl, H. Vrieling, K. A. Miller, G. L. Wolff, and G. S. Barsh, "Neomorphic agouti mutations in obese yellow mice," Nature Genetics, vol. 8, no. 1, pp. 59-65, Sep. 1994. DOI: 10.1038/ng0994-59.

[13] T. Jenuwein and C. D. Allis, "Translating the Histone Code," Science, vol. 293, no. 5532, pp. 1074-1080, Oct. 2001. DOI: 10.1126/science.1063127.

[14] G. Felsenfeld, "Quantitative approaches to problems of eukaryotic gene expression," Biophysical Chemistry, Special Issue in honor of John T. Edsall (1902-2002), vol. 100, no. 1-3, pp. 607-613, Dec. 2002. DOI: 10.1016/S03014622(02)00309-5.

[15] K. Sandman, S. L. Pereira, and J. N. Reeve, "Diversity of prokaryotic chromosomal proteins and the origin of the nucleosome," Cellular and Molecular 
Life Sciences CMLS, vol. 54, no. 12, pp. 1350-1364, Dec. 1998. DoI: 10.1007/ s000180050259.

[16] C. A. Ouzounis and N. C. Kyrpides, "Parallel origins of the nucleosome core and eukaryotic transcription from Archaea," Journal of Molecular Evolution, vol. 42, no. 2, pp. 234-239, Feb. 1996. DOI: 10.1007/BF02198849.

[17] K. A. Bailey and J. N. Reeve, "DNA repeats and archaeal nucleosome positioning," Research in Microbiology, vol. 150, no. 9-10, pp. 701-709, Nov. 1999. DOI: $10.1016 /$ S0923-2508(99)00122-9.

[18] M. E. Dinger, G. J. Baillie, and D. R. Musgrave, "Growth phase-dependent expression and degradation of histones in the thermophilic archaeon Thermococcus zilligii," Molecular microbiology, vol. 36, no. 4, pp. 876-885, 2000. DOI: $10.1046 / j .1365-2958.2000 .01904 . x$.

[19] J. Fillingham and J. F. Greenblatt, "A Histone Code for Chromatin Assembly," Cell, vol. 134, no. 2, pp. 206-208, Jul. 2008. Dor: 10 . 1016/j . cell . 2008.07 .007$.

[20] C. L. Peterson and M.-A. Laniel, "Histones and histone modifications," Current Biology, vol. 14, no. 14, R546-R551, Jul. 2004. DOI: $10.1016 /$ j . cub . 2004.07 .007$.

[21] A. P. Wolffe and J. J. Hayes, "Chromatin disruption and modification," Nucleic Acids Research, vol. 27, no. 3, pp. 711-720, Jan. 1999. DOI: 10.1093/nar/27. 3.711 .

[22] T. E. P. Consortium, "An integrated encyclopedia of DNA elements in the human genome," Nature, vol. 489, no. 7414, pp. 57-74, Sep. 2012. Dor: 10. 1038/nature11247. 
[23] C. L. Woodcock and R. P. Ghosh, "Chromatin Higher-order Structure and Dynamics," Cold Spring Harbor Perspectives in Biology, vol. 2, no. 5, May 2010. DOI: $10.1101 /$ cshperspect.a000596.

[24] S. A. Grigoryev, G. Arya, S. Correll, C. L. Woodcock, and T. Schlick, "Evidence for heteromorphic chromatin fibers from analysis of nucleosome interactions," Proceedings of the National Academy of Sciences, vol. 106, no. 32, pp. 13 317-13 322, Aug. 2009. DOI: 10.1073/pnas. 0903280106.

[25] K. Maeshima, S. Hihara, and M. Eltsov, "Chromatin structure: does the 30-nm fibre exist in vivo?" Current Opinion in Cell Biology, vol. 22, no. 3, pp. 291297, Jun. 2010. DOI: 10.1016/j.ceb.2010.03.001.

[26] R. K. Suto, R. S. Edayathumangalam, C. L. White, C. Melander, J. M. Gottesfeld, P. B. Dervan, and K. Luger, "Crystal Structures of Nucleosome Core Particles in Complex with Minor Groove DNA-binding Ligands," Journal of Molecular Biology, vol. 326, no. 2, pp. 371-380, Feb. 2003. DOI: $10.1016 /$ S0022-2836(02) 01407-9.

[27] F. Song, P. Chen, D. Sun, M. Wang, L. Dong, D. Liang, R.-M. Xu, P. Zhu, and G. Li, "Cryo-EM Study of the Chromatin Fiber Reveals a Double Helix Twisted by Tetranucleosomal Units," Science, vol. 344, no. 6182, pp. 376-380, Apr. 2014. DOI: 10.1126/science. 1251413.

[28] Y. Chen, J. M. Tokuda, T. Topping, J. L. Sutton, S. P. Meisburger, S. A. Pabit, L. M. Gloss, and L. Pollack, "Revealing transient structures of nucleosomes as DNA unwinds," Nucleic Acids Research, gku562, Jul. 2014. DOI: 10.1093/ nar/gku562.

[29] K. Andresen, I. Jimenez-Useche, S. C. Howell, C. Yuan, and X. Qiu, "Solution Scattering and FRET Studies on Nucleosomes Reveal DNA Unwrapping 
Effects of H3 and H4 Tail Removal," PLoS ONE, vol. 8, no. 11, M. FernandezZapico, Ed., e78587, Nov. 2013. DOI: 10.1371/journal.pone.0078587.

[30] S. C. Howell, K. Andresen, I. Jimenez-Useche, C. Yuan, and X. Qiu, "Elucidating Internucleosome Interactions and the Roles of Histone Tails," Biophysical Journal, vol. 105, no. 1, pp. 194-199, Jul. 2013. DOI: 10.1016/j . bpj . 2013. 05.021.

[31] R. Buning and J. van Noort, "Single-pair FRET experiments on nucleosome conformational dynamics," Biochimie, Special section "DNA and Chromosomes: Physical and Biological Approaches"Fifth multidisciplinary international summer school on DNA and chromosomes, vol. 92, no. 12, pp. 17291740, Dec. 2010. DOI: 10.1016/j.biochi.2010.08.010.

[32] M. Hammermann, K. Tóth, C. Rodemer, W. Waldeck, R. P. May, and J. Langowski, "Salt-dependent compaction of di-and trinucleosomes studied by small-angle neutron scattering," Biophysical journal, vol. 79, no. 1, pp. 584594, 2000. DOI: $10.1016 /$ S0006-3495(00)76318-1.

[33] H.-X. Zhou, G. Rivas, and A. P. Minton, "Macromolecular Crowding and Confinement: Biochemical, Biophysical, and Potential Physiological Consequences," Annual Review of Biophysics, vol. 37, no. 1, pp. 375-397, Jun. 2008. DOI: $10.1146 /$ annurev . biophys.37.032807.125817.

[34] B. Poolman and E. Glaasker, "Regulation of compatible solute accumulation in bacteria," Molecular Microbiology, vol. 29, no. 2, pp. 397-407, Jul. 1998. DOI: $10.1046 / j .1365-2958.1998 .00875 . x$.

[35] D. J. Clark and T. Kimura, "Electrostatic mechanism of chromatin folding," Journal of Molecular Biology, vol. 211, no. 4, pp. 883-896, Feb. 1990. DOI: $10.1016 / 0022-2836(90) 90081-V$. 
[36] J. Widom, "Physicochemical studies of the folding of the $100 \AA$ nucleosome filament into the $300 \AA$ filament," Journal of Molecular Biology, vol. 190, no. 3, pp. 411-424, Aug. 1986. DOI: 10.1016/0022-2836(86)90012-4.

[37] D. Sen and D. M. Crothers, "Condensation of chromatin: role of multivalent cations," Biochemistry, vol. 25, no. 7, pp. 1495-1503, Apr. 1986. DOI: 10 . $1021 /$ bi00355a004.

[38] J. C. Hansen, "Conformational Dynamics of the Chromatin Fiber in Solution: Determinants, Mechanisms, and Functions," Annual Review of Biophysics and Biomolecular Structure, vol. 31, no. 1, pp. 361-392, Jun. 2002. DOI: 10.1146/ annurev . biophys.31.101101.140858.

[39] J. Widom, "Structure, Dynamics, and Function of Chromatin in Vitro," $A n$ nual Review of Biophysics and Biomolecular Structure, vol. 27, no. 1, pp. 285327, Jun. 1998. DOI: 10.1146/annurev .biophys.27.1.285.

[40] B. Dorigo, T. Schalch, K. Bystricky, and T. J. Richmond, "Chromatin Fiber Folding: Requirement for the Histone H4 N-terminal Tail," Journal of Molecular Biology, vol. 327, no. 1, pp. 85-96, Mar. 2003. DOI: 10.1016/S0022$2836(03) 00025-1$.

[41] M. de Frutos, E. Raspaud, A. Leforestier, and F. Livolant, "Aggregation of Nucleosomes by Divalent Cations," Biophysical Journal, vol. 81, no. 2, pp. 11271132, Aug. 2001. DOI: 10.1016/S0006-3495(01)75769-4.

[42] V. A. Bloomfield, "DNA condensation," Current Opinion in Structural Biology, vol. 6, no. 3, pp. 334-341, Jun. 1996. DOI: 10.1016 /S0959-440X (96) 80052-2.

[43] J. Sun, Q. Zhang, and T. Schlick, "Electrostatic mechanism of nucleosomal array folding revealed by computer simulation," Proceedings of the National 
Academy of Sciences, vol. 102, no. 23, pp. 8180-8185, Jun. 2005. DOI: 10 . 1073/pnas. 0408867102.

[44] K. Luger, T. J. Rechsteiner, A. J. Flaus, M. M. Waye, and T. J. Richmond, "Characterization of nucleosome core particles containing histone proteins made in bacteria," Journal of molecular biology, vol. 272, no. 3, pp. 301-311, 1997. DOI: $10.1006 / j m b i .1997 .1235$.

[45] C. A. Davey and T. J. Richmond, "DNA-Dependent Divalent Cation Binding in the Nucleosome Core Particle," Proceedings of the National Academy of Sciences of the United States of America, vol. 99, no. 17, pages, 2002. Dor: 10.1073/pnas. 172271399 .

[46] J. Widom, "Role of DNA sequence in nucleosome stability and dynamics," Quarterly Reviews of Biophysics, vol. 34, no. 3, pp. 269-324, Aug. 2001. DoI: $10.1016 / j . \operatorname{cell} .2015 .02 .001$.

[47] A. Routh, S. Sandin, and D. Rhodes, "Nucleosome repeat length and linker histone stoichiometry determine chromatin fiber structure," Proceedings of the National Academy of Sciences, vol. 105, no. 26, pp. 8872-8877, 2008. DOI: 10.1073/pnas. 0802336105 .

[48] H. J. Szerlong and J. C. Hansen, "Nucleosome distribution and linker DNA: connecting nuclear function to dynamic chromatin structure," Biochemistry $\&$ Cell Biology, vol. 89, no. 1, pp. 24-34, Feb. 2011. DoI: 10.1139/010-139.

[49] G. Arya, Q. Zhang, and T. Schlick, "Flexible Histone Tails in a New Mesoscopic Oligonucleosome Model," Biophysical Journal, vol. 91, no. 1, pp. 133-150, Jul. 2006. DOI: $10.1529 /$ biophysj.106.083006.

[50] F. Gordon, K. Luger, and J. C. Hansen, "The Core Histone N-terminal Tail Domains Function Independently and Additively during Salt-dependent Oligomer- 
ization of Nucleosomal Arrays," Journal of Biological Chemistry, vol. 280, no. 40, pp. 33 701-33 706, Oct. 2005. DOI: 10.1074/jbc.M507048200.

[51] W.-J. Shia, S. G. Pattenden, and J. L. Workman, "Histone H4 lysine 16 acetylation breaks the genome's silence," Genome biology, vol. 7, no. 5, p. 217, 2006. DOI: $10.1186 / \mathrm{gb}-2006-7-5-217$.

[52] A. Allahverdi, R. Yang, N. Korolev, Y. Fan, C. A. Davey, C.-F. Liu, and L. Nordenskiold, "The effects of histone H4 tail acetylations on cation-induced chromatin folding and self-association," Nucleic Acids Research, vol. 39, no. 5, pp. 1680-1691, Mar. 2011. DOI: 10.1093/nar/gkq900.

[53] P. J. Robinson, W. An, A. Routh, F. Martino, L. Chapman, R. G. Roeder, and D. Rhodes, "30 nm Chromatin Fibre Decompaction Requires both H4K16 Acetylation and Linker Histone Eviction," Journal of Molecular Biology, vol. 381, no. 4, pp. 816-825, Sep. 2008. DOI: 10.1016/j.jmb.2008.04.050.

[54] M. Shogren-Knaak, "Histone H4-K16 Acetylation Controls Chromatin Structure and Protein Interactions," Science, vol. 311, no. 5762, pp. 844-847, Feb. 2006. DOI: $10.1126 /$ science. 1124000 .

[55] N. Korolev, Y. Zhao, A. Allahverdi, K. D. Eom, J. P. Tam, and L. Nordenskiöld, "The effect of salt on oligocation-induced chromatin condensation," Biochemical and Biophysical Research Communications, vol. 418, no. 2, pp. 205210, Feb. 2012. DOI: 10.1016/j.bbrc.2011.12.112.

[56] M. Tark-Dame, R. van Driel, and D. W. Heermann, "Chromatin folding - from biology to polymer models and back," Journal of Cell Science, vol. 124, no. 6, pp. 839-845, Mar. 2011. DOI: $10.1242 /$ jcs. 077628.

[57] N. Korolev, A. P. Lyubartsev, and L. Nordenskiöld, "Cation-induced polyelectrolyte-polyelectrolyte attraction in solutions of DNA and nucleosome core 
particles," Advances in Colloid and Interface Science, Polyelectrolytes, vol. 158, no. 1-2, pp. 32-47, Jul. 2010. DOI: 10.1016/j.cis.2009.08.002.

[58] R. Dootz, A. C. Toma, and T. Pfohl, "Structural and dynamic properties of linker histone H1 binding to DNA," Biomicrofluidics, vol. 5, no. 2, p. 024 104, 2011. DOI: $10.1063 / 1.3587096$.

[59] I. M. Kulić and H. Schiessel, "Chromatin Dynamics: Nucleosomes go Mobile through Twist Defects," Physical Review Letters, vol. 91, no. 14, p. 148103 , Oct. 2003. DOI: 10.1103/PhysRevLett.91.148103.

[60] P. M. Diesinger and D. W. Heermann, "Monte Carlo Simulations indicate that Chromati: Nanostructure is accessible by Light Microscopy," PMC Biophysics, vol. 3 , no. 1, p. 11, 2010. DOI: 10.1186/1757-5036-3-11.

[61] X. Zuo, A. Grishaev, and J. Wang. (Oct. 2009). The First NIH Workshop on Small Angle X-ray Scattering and Application in Biomolecular Studies.

[62] K. Luger, A. W. Mäder, R. K. Richmond, D. F. Sargent, and T. J. Richmond, "Crystal structure of the nucleosome core particle at $2.8 \AA$ resolution," Nature, vol. 389 , no. 6648 , pp. 251-260, Sep. 1997. DOI: 10.1038/38444.

[63] H. Niederstrasser. (Mar. 2004). Electron Microscopy: Freezing Samples, [Online]. Available: http://www. snaggledworks.com/em_for_dummies/freeze. html (visited on 09/21/2015).

[64] (Jan. 2012). Integrating SAXS/NMR Data for Structure Determination, [Online]. Available: http://www . theresonance. com/integrating-saxs-and$\mathrm{nmr} /$ (visited on 09/22/2015).

[65] D. Marion, "An introduction to biological NMR spectroscopy," Molecular $\mathscr{E}$ Cellular Proteomics, mcp.O113.030239, Jul. 2013. DOI: 10.1074/mcp.0113. 030239. 
[66] X. Qiu, K. Andresen, J. S. Lamb, L. W. Kwok, and L. Pollack, "Abrupt Transition from a Free, Repulsive to a Condensed, Attractive DNA Phase, Induced by Multivalent Polyamine Cations," Physical Review Letters, vol. 101, no. 22, Nov. 2008. DOI: 10.1103/PhysRevLett.101.228101.

[67] X. Qiu, K. Andresen, L. W. Kwok, J. S. Lamb, H. Y. Park, and L. Pollack, "Inter-DNA Attraction Mediated by Divalent Counterions," Physical Review Letters, vol. 99, no. 3, Jul. 2007. DOI: 10.1103/PhysRevLett.99.038104.

[68] X. Qiu, L. Kwok, H. Park, J. Lamb, K. Andresen, and L. Pollack, "Measuring Inter-DNA Potentials in Solution," Physical Review Letters, vol. 96, no. 13, Apr. 2006. DOI: 10.1103/PhysRevLett.96.138101.

[69] D. I. Svergun, "Restoring low resolution structure of biological macromolecules from solution scattering using simulated annealing," Biophysical journal, vol. 76, no. 6, pp. 2879-2886, 1999. DOI: 10.1016/S0006-3495(99)77443-6.

[70] S. S. Nielsen, M. Møller, and R. E. Gillilan, "High-throughput biological smallangle X-ray scattering with a robotically loaded capillary cell," Journal of Applied Crystallography, vol. 45, no. 2, pp. 213-223, Apr. 2012. DOI: 10.1107/ S0021889812000957.

[71] C. J. Glinka, J. G. Barker, B. Hammouda, S. Krueger, J. J. Moyer, and W. J. Orts, "The $30 \mathrm{~m}$ small-angle neutron scattering instruments at the National Institute of Standards and Technology," Journal of Applied Crystallography, vol. 31, no. 3, pp. 430-445, 1998. DOI: 10.1107/S0021889897017020.

[72] O. Glatter and O. Kratky, Small Angle X-ray Scattering. Academic Press, 1982.

[73] A. Guinier and G. Fournet, Small-angle scattering of X-rays. Wiley, 1955.

[74] L. A. Feigin, D. I. Svergun, and G. W. Taylor, Structure Analysis by SmallAngle X-Ray and Neutron Scattering. 1987. 
[75] J. I. Langford and A. J. C. Wilson, "Scherrer after sixty years: A survey and some new results in the determination of crystallite size," Journal of Applied Crystallography, vol. 11, no. 2, pp. 102-113, Apr. 1978. DOI: 10.1107/ S0021889878012844.

[76] X. Qiu, J. Giannini, S. C. Howell, Q. Xia, F. Ke, and K. Andresen, "Ion Competition in Condensed DNA Arrays in the Attractive Regime," Biophysical Journal, vol. 105, no. 4, pp. 984-992, Aug. 2013. DOI: 10.1016/j.bpj. 2013. 07.004.

[77] X. Qiu, C. Y. Khripin, F. Ke, S. C. Howell, and M. Zheng, "Electrostatically Driven Interactions between Hybrid DNA-Carbon Nanotubes," Physical Review Letters, vol. 111, no. 4, Jul. 2013. DOI: 10.1103/PhysRevLett. 111. 048301.

[78] X. Qiu, D. C. Rau, V. A. Parsegian, L. T. Fang, C. M. Knobler, and W. M. Gelbart, "Salt-Dependent DNA-DNA Spacings in Intact Bacteriophage $\lambda$ Reflect Relative Importance of DNA Self-Repulsion and Bending Energies," Physical Review Letters, vol. 106, no. 2, p. 028102 , Jan. 2011. DOI: 10.1103 / PhysRevLett.106.028102.

[79] N. Korolev, A. Allahverdi, Y. Yang, Y. Fan, A. P. Lyubartsev, and L. Nordenskiöld, "Electrostatic Origin of Salt-Induced Nucleosome Array Compaction," Biophysical Journal, vol. 99, no. 6, pp. 1896-1905, Sep. 2010. DOI: 10.1016/ j.bpj.2010.07.017.

[80] N. Korolev, Y. Fan, A. P. Lyubartsev, and L. Nordenskiöld, "Modelling chromatin structure and dynamics: status and prospects," Current Opinion in Structural Biology, vol. 22, no. 2, pp. 151-159, Apr. 2012. DOI: 10.1016/j . sbi.2012.01.006. 
[81] T. J. Richmond and C. A. Davey, "The structure of DNA in the nucleosome core," Nature, vol. 423, no. 6936, pp. 145-150, 2003. DOI: $10.1038 /$ nature01595.

[82] H. H. Gan and T. Schlick, "Chromatin Ionic Atmosphere Analyzed by a Mesoscale Electrostatic Approach," Biophysical Journal, vol. 99, no. 8, pp. 25872596, Oct. 2010. DOI: 10.1016/j.bpj .2010.08.023.

[83] C. K. Materese, A. Savelyev, and G. A. Papoian, "Counterion Atmosphere and Hydration Patterns near a Nucleosome Core Particle," Journal of the American Chemical Society, vol. 131, no. 41, pp. 15005-15013, Oct. 2009. DOI: $10.1021 / \mathrm{ja} 905376 \mathrm{q}$.

[84] D. A. Potoyan and G. A. Papoian, "Energy Landscape Analyses of Disordered Histone Tails Reveal Special Organization of Their Conformational Dynamics," Journal of the American Chemical Society, vol. 133, no. 19, pp. 74057415, May 2011. DOI: $10.1021 /$ ja1111964.

[85] G. Arya and T. Schlick, "A Tale of Tails: How Histone Tails Mediate Chromatin Compaction in Different Salt and Linker Histone Environments†," The Journal of Physical Chemistry A, vol. 113, no. 16, pp. 4045-4059, Apr. 2009. DOI: $10.1021 / j p 810375 d$.

[86] F. Mühlbacher, H. Schiessel, and C. Holm, "Tail-induced attraction between nucleosome core particles," Physical Review E, vol. 74, no. 3, p. 031919, Sep. 2006. DOI: $10.1103 /$ PhysRevE.74.031919.

[87] P. M. Diesinger and D. W. Heermann, "Depletion Effects Massively Change Chromatin Properties and Influence Genome Folding," Biophysical Journal, vol. 97, no. 8, pp. 2146-2153, Oct. 2009. DOI: 10.1016/j.bpj.2009.06.057.

[88] R. P. Hjelm, G. G. Kneale, P. Suau, J. P. Baldwin, E. M. Bradbury, and K. Ibel, "Small angle neutron scattering studies of chromatin subunits in solution," 
Cell, vol. 10, no. 1, pp. 139-151, Jan. 1977. DoI: 10.1016/0092-8674(77) 90148-9.

[89] S. H. Chen, E. Y. Sheu, J. Kalus, and H. Hoffman, "Small-angle neutron scattering investigation of correlations in charged macromolecular and supramolecular solutions," Journal of Applied Crystallography, vol. 21, no. 6, pp. 751-769, Dec. 1988. DOI: $10.1107 /$ S0021889888008052.

[90] A. Bertin, M. Renouard, J. S. Pedersen, F. Livolant, and D. Durand, "H3 and H4 Histone Tails Play a Central Role in the Interactions of Recombinant NCPs," Biophysical Journal, vol. 92, no. 7, pp. 2633-2645, Apr. 2007. DOI: 10.1529/biophysj.106.093815.

[91] T. Yager and K. E. van Holde, "Dynamics and equilibria of nucleosomes at elevated ionic strength," Journal of Biological Chemistry, vol. 259, no. 7, pp. 4212-4222, Oct. 1984.

[92] C. Yang, M. J. van der Woerd, U. M. Muthurajan, J. C. Hansen, and K. Luger, "Biophysical analysis and small-angle X-ray scattering-derived structures of MeCP2-nucleosome complexes," Nucleic Acids Research, vol. 39, no. 10, pp. 4122-4135, May 2011. DOI: 10.1093/nar/gkr005.

[93] S. Mangenot, A. Leforestier, P. Vachette, D. Durand, and F. Livolant, "SaltInduced Conformation and Interaction Changes of Nucleosome Core Particles," Biophysical Journal, vol. 82, no. 1, pp. 345-356, Jan. 2002. DOI: 10. 1016/S0006-3495 (02) 75399-X.

[94] Y. Liu, E. Fratini, P. Baglioni, W.-R. Chen, and S.-H. Chen, "Effective LongRange Attraction between Protein Molecules in Solutions Studied by Small Angle Neutron Scattering," Physical Review Letters, vol. 95, no. 11, p. 118 102, Sep. 2005. DOI: 10.1103/PhysRevLett.95.118102. 
[95] E. Trizac, L. Bocquet, M. Aubouy, and H. H. von Grünberg, "Alexander's Prescription for Colloidal Charge Renormalization," Langmuir, vol. 19, no. 9, pp. 4027-4033, Apr. 2003. DOI: 10.1021/la027056m.

[96] S. Mangenot, E. Raspaud, C. Tribet, L. Belloni, and F. Livolant, "Interactions between isolated nucleosome core particles: A tail-bridging effect?" The European Physical Journal E: Soft Matter and Biological Physics, vol. 7, no. 3, pp. 221-231, 2002. DOI: 10.1140/epje/i200101151.

[97] G. Arya and T. Schlick, "Role of histone tails in chromatin folding revealed by a mesoscopic oligonucleosome model," Proceedings of the National Academy of Sciences, vol. 103, no. 44, pp. 16 236-16241, Oct. 2006. DOI: 10.1073/pnas . 0604817103.

[98] N. Korolev and L. Nordenskiöld, "H4 histone tail mediated DNA-DNA interaction and effects on DNA structure, flexibility, and counterion binding. A molecular dynamics study," Biopolymers, vol. 86, no. 5-6, pp. 409-423, Aug. 2007. DOI: 10.1002/bip. 20749.

[99] B. Dorigo, T. Schalch, A. Kulangara, S. Duda, R. R. Schroeder, and T. J. Richmond, "Nucleosome Arrays Reveal the Two-Start Organization of the Chromatin Fiber," Science, vol. 306, no. 5701, pp. 1571-1573, Nov. 2004. DOI: 10.1126/science.1103124.

[100] P. N. Dyer, R. S. Edayathumangalam, C. L. White, Y. Bao, S. Chakravarthy, U. M. Muthurajan, and K. Luger, "Reconstitution of nucleosome core particles from recombinant histones and DNA," Methods in Enzymology, vol. 375, pp. 23-44, 2004. DOI: $10.1016 /$ S0076-6879(03) 75002-2.

[101] S. G. Grant, J. Jessee, F. R. Bloom, and D. Hanahan, "Differential plasmid rescue from transgenic mouse DNAs into Escherichia coli methylation-restriction 
mutants.," Proceedings of the National Academy of Sciences, vol. 87, no. 12, pp. 4645-4649, 1990. DOI: 10.1073/pnas.87.12.4645.

[102] I. Syrový and Z. Hodný, "Staining and quantification of proteins separated by polyacrylamide gel electrophoresis," Journal of Chromatography B: Biomedical Sciences and Applications, vol. 569, no. 1, pp. 175-196, Sep. 1991. DOI: 10. 1016/0378-4347 (91)80229-6.

[103] L. Yang, "Using an in-vacuum CCD detector for simultaneous small- and wideangle scattering at beamline X9," Journal of Synchrotron Radiation, vol. 20, no. 2, pp. 211-218, Mar. 2013. DOI: 10.1107/S0909049512048984.

[104] B. R. Pauw, "Everything SAXS: small-angle scattering pattern collection and correction," Journal of Physics: Condensed Matter, vol. 25, no. 38, p. 383201 , Sep. 2013. DOI: $10.1088 / 0953-8984 / 25 / 38 / 383201$.

[105] G. Hura, J. M. Sorenson, R. M. Glaeser, and T. Head-Gordon, "A high-quality x-ray scattering experiment on liquid water at ambient conditions," The Journal of Chemical Physics, vol. 113, no. 20, pp. 9140-9148, Nov. 2000. DoI: $10.1063 / 1.1319614$.

[106] K. N. Dyer, M. Hammel, R. P. Rambo, S. E. Tsutakawa, I. Rodic, S. Classen, J. A. Tainer, and G. L. Hura, "High-Throughput SAXS for the Characterization of Biomolecules in Solution: A Practical Approach," Methods in molecular biology (Clifton, N.J.), vol. 1091, pp. 245-258, 2014. DOI: $10.1007 / 978-1-$ 62703-691-7_18.

[107] D. I. Svergun, "Determination of the regularization parameter in indirecttransform methods using perceptual criteria," Journal of Applied Crystallography, vol. 25, no. 4, pp. 495-503, Aug. 1992. DOI: 10.1107/S0021889892001663. 
[108] D. Schneidman-Duhovny, M. Hammel, and A. Sali, "FoXS: a web server for rapid computation and fitting of SAXS profiles," Nucleic Acids Research, vol. 38, no. suppl 2, W540-W544, Jan. 2010. DOI: 10.1093/nar/gkq461.

[109] J. E. Curtis, S. Raghunandan, H. Nanda, and S. Krueger, "SASSIE: A program to study intrinsically disordered biological molecules and macromolecular ensembles using experimental scattering restraints," Computer Physics Communications, vol. 183, no. 2, pp. 382-389, Feb. 2012. DOI: 10.1016/j.cpc . 2011.09 .010 .

[110] W. K. Olson, M. Bansal, S. K. Burley, R. E. Dickerson, M. Gerstein, S. C. Harvey, U. Heinemann, X.-J. Lu, S. Neidle, Z. Shakked, H. Sklenar, M. Suzuki, C.-S. Tung, E. Westhof, C. Wolberger, and H. M. Berman, "A standard reference frame for the description of nucleic acid base-pair geometry," Journal of Molecular Biology, vol. 313, no. 1, pp. 229-237, Oct. 2001. DOI: 10.1006/ jmbi.2001.4987.

[111] K. F. Smith, R. A. Harrison, and S. J. Perkins, "Structural comparisons of the native and reactive-centre-cleaved forms of $\alpha_{1}$-antitrypsin by neutron- and Xray-scattering in solution," Biochemical Journal, vol. 267, no. 1, pp. 203-212, Apr. 1990. DOI: 10.1042/bj2670203.

[112] R. E. Franklin and R. G. Gosling, "Molecular configuration in sodium thymonucleate," Nature, vol. 171, no. 4356, pp. 740-741, Apr. 1953. DOI: 10 . 1038/171740a0.

[113] M. H. F. Wilkins, A. R. Stokes, and H. R. Wilson, "Molecular structure of deoxypentose nucleic acids," Nature, vol. 171, no. 4356, pp. 738-740, Apr. 1953. DOI: $10.1038 / 171738 \mathrm{a} 0$. 
[114] M. V. Petoukhov and D. I. Svergun, "Global rigid body modeling of macromolecular complexes against small-angle scattering data," Biophysical Journal, vol. 89, no. 2, pp. 1237-1250, Aug. 2005. DOI: 10.1529/biophysj .105. 064154.

[115] P. V. Konarev, M. V. Petoukhov, and D. I. Svergun, "MASSHA-a graphics system for rigid-body modelling of macromolecular complexes against solution scattering data," Journal of Applied Crystallography, vol. 34, no. 4, pp. 527532, 2001. DOI: $10.1107 /$ S0021889801006100.

[116] E. Benson, A. Mohammed, J. Gardell, S. Masich, E. Czeizler, P. Orponen, and B. Högberg, "DNA rendering of polyhedral meshes at the nanoscale," Nature, vol. 523, no. 7561, pp. 441-444, Jul. 2015. DOI: 10.1038/nature14586.

[117] A. Savelyev and A. D. MacKerell, "All-atom polarizable force field for DNA based on the classical drude oscillator model," Journal of Computational Chemistry, vol. 35, no. 16, pp. 1219-1239, Jun. 2014. DOI: 10.1002/jcc.23611.

[118] A. Savelyev and G. A. Papoian, "Chemically accurate coarse graining of doublestranded DNA," Proceedings of the National Academy of Sciences, vol. 107, no. 47 , pp. $20340-20345$, 2010. DOI: 10.1073/pnas. 1001163107.

[119] P. Šulc, F. Romano, T. E. Ouldridge, L. Rovigatti, J. P. K. Doye, and A. A. Louis, "Sequence-dependent thermodynamics of a coarse-grained DNA model," The Journal of Chemical Physics, vol. 137, no. 13, p. 135 101, 2012. DOI: $10.1063 / 1.4754132$.

[120] J. J. de Pablo, "Coarse-Grained Simulations of Macromolecules: From DNA to Nanocomposites," Annual Review of Physical Chemistry, vol. 62, no. 1, pp. 555-574, May 2011. DOI: 10.1146/annurev-physchem-032210-103458.

[121] T. A. Knotts, N. Rathore, D. C. Schwartz, and J. J. de Pablo, "A coarse grain model for DNA," The Journal of Chemical Physics, vol. 126, no. 8, p. 084 901, 2007. DOI: $10.1063 / 1.2431804$. 
[122] A. Morriss-Andrews, J. Rottler, and S. S. Plotkin, "A systematically coarsegrained model for DNA and its predictions for persistence length, stacking, twist, and chirality," The Journal of Chemical Physics, vol. 132, no. 3, p. 035 105, 2010. DOI: $10.1063 / 1.3269994$.

[123] P. D. Dans, A. Zeida, M. R. Machado, and S. Pantano, "A Coarse Grained Model for Atomic-Detailed DNA Simulations with Explicit Electrostatics," Journal of Chemical Theory and Computation, vol. 6, no. 5, pp. 1711-1725, May 2010. DOI: 10.1021/ct900653p.

[124] D. Franke and D. I. Svergun, "DAMMIF , a program for rapid ab-initio shape determination in small-angle scattering," Journal of Applied Crystallography, vol. 42, no. 2, pp. 342-346, Apr. 2009. DOI: 10.1107/S0021889809000338.

[125] D. Norouzi and V. B. Zhurkin, "Topological Polymorphism of the Two-Start Chromatin Fiber," Biophysical Journal, vol. 108, no. 10, pp. 2591-2600, May 2015. DOI: $10.1016 / \mathrm{j} . \mathrm{bpj} .2015 .04 .015$.

[126] Y. Wang, D. R. Tree, and K. D. Dorfman, "Simulation of DNA Extension in Nanochannels," Macromolecules, vol. 44, no. 16, pp. 6594-6604, Aug. 2011. DOI: $10.1021 / \mathrm{ma} 201277 \mathrm{e}$.

[127] N. J. Clark, H. Zhang, S. Krueger, H. J. Lee, R. R. Ketchem, B. Kerwin, S. R. Kanapuram, M. J. Treuheit, A. McAuley, and J. E. Curtis, "Small-Angle Neutron Scattering Study of a Monoclonal Antibody Using Free-Energy Constraints," The Journal of Physical Chemistry B, vol. 117, no. 45, pp. $14029-$ 14 038, Nov. 2013. DOI: $10.1021 / j p 408710 r$.

[128] N. J. Clark, M. Kramer, U. M. Muthurajan, and K. Luger, "Alternative Modes of Binding of Poly(ADP-ribose) Polymerase 1 to Free DNA and Nucleosomes," Journal of Biological Chemistry, vol. 287, no. 39, pp. 32 430-32 439, Sep. 2012. DOI: $10.1074 / \mathrm{jbc} . \mathrm{M} 112.397067$. 
[129] J. Wang and H. Gao, "A generalized bead-rod model for Brownian dynamics simulations of wormlike chains under strong confinement," The Journal of Chemical Physics, vol. 123, no. 8, p. 084906, Aug. 2005. DOI: 10.1063/1. 2008233.

[130] C. Bustamante, J. F. Marko, E. D. Siggia, and S. Smith, "Entropic Elasticity of Lambda-Phage DNA," Science, vol. 265, no. 5178, pp. 1599-1600, Sep. 1994. DOI: $10.1126 /$ science. 8079175.

[131] S. B. Smith, L. Finzi, and C. Bustamante, "Direct mechanical measurements of the elasticity of single DNA molecules by using magnetic beads," Science (New York, N.Y.), vol. 258, no. 5085, pp. 1122-1126, Nov. 1992. DOI: 10. 1126/science. 1439819 .

[132] W. K. Olson, A. A. Gorin, X.-J. Lu, L. M. Hock, and V. B. Zhurkin, "DNA sequence-dependent deformability deduced from protein-DNA crystal complexes," Proceedings of the National Academy of Sciences, vol. 95, no. 19, pp. $11163-11168$, 1998. DOI: 10.1073/pnas.95.19.11163.

[133] D. Schneidman-Duhovny, M. Hammel, J. A. Tainer, and A. Sali, "Accurate SAXS Profile Computation and its Assessment by Contrast Variation Experiments," Biophysical Journal, vol. 105, no. 4, pp. 962-974, Aug. 2013. DOI: 10.1016/j.bpj.2013.07.020.

[134] J. R. C. van der Maarel, Introduction to biopolymer physics. Hackensack, N.J.: World Scientific, 2007.

[135] J. E. Godfrey and H. Eisenberg, "The flexibility of low molecular weight double-stranded dna as a function of length: II. Light scattering measurements and the estimation of persistence lengths from light scattering, sedimentation and viscosity," Biophysical Chemistry, vol. 5, no. 3, pp. 301-318, Sep. 1976. DOI: $10.1016 / 0301-4622(76) 80042-7$. 
[136] B. Schneider, S. Neidle, and H. M. Berman, "Conformations of the sugarphosphate backbone in helical DNA crystal structures," Biopolymers, vol. 42, no. 1, pp. 113-124, Jul. 1997. DOI: 10.1002/(SICI) 1097-0282(199707) 42: 1<113: :AID-BIP10>3.0.CO;2-0.

[137] G. A. Leonard and W. N. Hunter, "Crystal and molecular structure of d(CGTA GATCTACG) at $2.25 \AA$ resolution," J.Mol.Biol., vol. 234, pp. 198-208, Apr. 1993. DOI: $10.1006 / j m b i .1993 .1574$.

[138] K. Hart, N. Foloppe, C. M. Baker, E. J. Denning, L. Nilsson, and A. D. MacKerell, "Optimization of the CHARMM Additive Force Field for DNA: Improved Treatment of the BI/BII Conformational Equilibrium," Journal of Chemical Theory and Computation, vol. 8, no. 1, pp. 348-362, Jan. 2012. DOI: $10.1021 /$ ct200723y.

[139] M. van Dijk and A. M. J. J. Bonvin, "3D-DART: a DNA structure modelling server," Nucleic Acids Research, vol. 37, W235-W239, Jul. 2009. DOI: 10 . 1093/nar/gkp287.

[140] G. H. Golub and C. F. V. Loan, Matrix Computations, 4th ed. JHU Press, Dec. 2012.

[141] C. D. Putnam, M. Hammel, G. L. Hura, and J. A. Tainer, "X-ray solution scattering (SAXS) combined with crystallography and computation: defining accurate macromolecular structures, conformations and assemblies in solution," Quarterly Reviews of Biophysics, vol. 40, no. 03, pp. 191-285, Aug. 2007. DOI: 10.1017/S0033583507004635.

[142] A. V. Sokolova, V. V. Volkov, and D. I. Svergun, "Prototype of a database for rapid protein classification based on solution scattering data," Journal of applied crystallography, vol. 36, no. 3, pp. 865-868, 2003. DOI: $10.1107 /$ S0021889803000220. 
[143] C. E. Shannon and W. Weaver, The mathematical theory of communication. Urbana: University of Illinois Press, 1949.

[144] C. E. Shannon, "A mathematical theory of communication," vol. 5, no. 1, pp. 3-55, 1948. DOI: 10.1145/584091.584093.

[145] P. Chacón, F. Morán, J. F. Díaz, E. Pantos, and J. M. Andreu, "Low-Resolution Structures of Proteins in Solution Retrieved from X-Ray Scattering with a Genetic Algorithm," Biophysical Journal, vol. 74, no. 6, pp. 2760-2775, Jun. 1998. DOI: $10.1016 / \mathrm{S} 0006-3495$ (98) 77984-6.

[146] J. R. Taylor, An introduction to error analysis: the study of uncertainties in physical measurements, 2nd ed. Sausalito, Calif: University Science Books, 1997.

[147] P. B. Moore, "Small-angle scattering. Information content and error analysis," Journal of Applied Crystallography, vol. 13, no. 2, pp. 168-175, 1980. DOI: 10.1107/S002188988001179X.

[148] M. H. J. Koch, P. Vachette, and D. I. Svergun, "Small-angle scattering: a view on the properties, structures and structural changes of biological macromolecules in solution," Quarterly Reviews of Biophysics, vol. 36, no. 02, pp. 147 227, May 2003. DOI: 10.1017/S0033583503003871.

[149] D. Svergun, C. Barberato, and M. H. J. Koch, "CRYSOL-a program to evaluate X-ray solution scattering of biological macromolecules from atomic coordinates," Journal of Applied Crystallography, vol. 28, no. 6, pp. 768-773, 1995. DOI: $10.1107 / \mathrm{S} 0021889895007047$.

[150] "Torsion angle," in IUPAC Compendium of Chemical Terminology, A. D. McNaught and A. Wilkinson, Eds., 2.1.0, Blackwell Scientific Publications, Oxford, 1997. 
[151] A. Gansen, A. Valeri, F. Hauger, S. Felekyan, S. Kalinin, K. Tóth, J. Langowski, and C. A. Seidel, "Nucleosome disassembly intermediates characterized by single-molecule FRET," Proceedings of the National Academy of Sciences, vol. 106, no. 36, pp. 15 308-15 313, 2009. DOI: 10.1073/pnas. 0903005106.

[152] J. Wu, Z. Wang, W. Lin, and S. Chen, "Investigation of the interaction between poly(ethylene glycol) and protein molecules using low field nuclear magnetic resonance," Acta Biomaterialia, vol. 9, no. 5, pp. 6414-6420, May 2013. DoI: $10.1016 / j$. actbio. 2013.01 .006 .

[153] J. C. Wang, "Helical repeat of DNA in solution," Proceedings of the National Academy of Sciences, vol. 76, no. 1, pp. 200-203, 1979. DOI: 10.1073/pnas . 76.1 .200 .

[154] J.-P. Wang, Y. Fondufe-Mittendorf, L. Xi, G.-F. Tsai, E. Segal, and J. Widom, "Preferentially Quantized Linker DNA Lengths in Saccharomyces cerevisiae," PLoS Computational Biology, vol. 4, no. 9, G. Stormo, Ed., e1000175, Sep. 2008. DOI: $10.1371 /$ journal.pcbi.1000175.

[155] Y. Liu, W.-R. Chen, and S.-H. Chen, "Cluster formation in two-Yukawa fluids," The Journal of Chemical Physics, vol. 122, no. 4, p. 044 507, Jan. 2005. DOI: $10.1063 / 1.1830433$.

[156] T. Zemb and V. A. Parsegian, "Editorial overview: Hydration forces," Current Opinion in Colloid 8$\}$ Interface Science, vol. 16, no. 6, pp. 515-516, Dec. 2011. DOI: $10.1016 / j$. cocis .2011 .06 .002 . 


\title{
Appendices
}

\section{A: Supplemental data for "Elucidating Internucleosome Inter- actions and the Roles of Histone Tails}

\author{
Steven C. Howell, ${ }^{\dagger}$ Kurt Andresen, ${ }^{\ddagger}$ Isabel Jimenez-Useche ${ }^{\S}$, Chongli Yuan, ${ }^{\S}$ and \\ Xiangyun Qiu ${ }^{\dagger}$ \\ †Department of Physics, George Washington University, Washington, DC; \\ ${ }^{\ddagger}$ Department of Physics, Gettysburg College, Gettysburg, Pennsylvania;

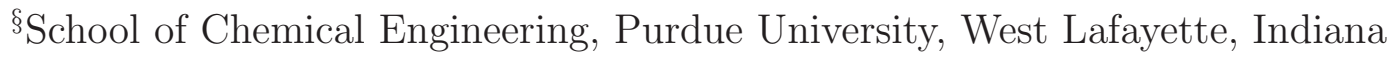 \\ Biophysical Journal Volume 105 July 2013 194-199
}


Supporting Data to the Results Presented in the Main Text

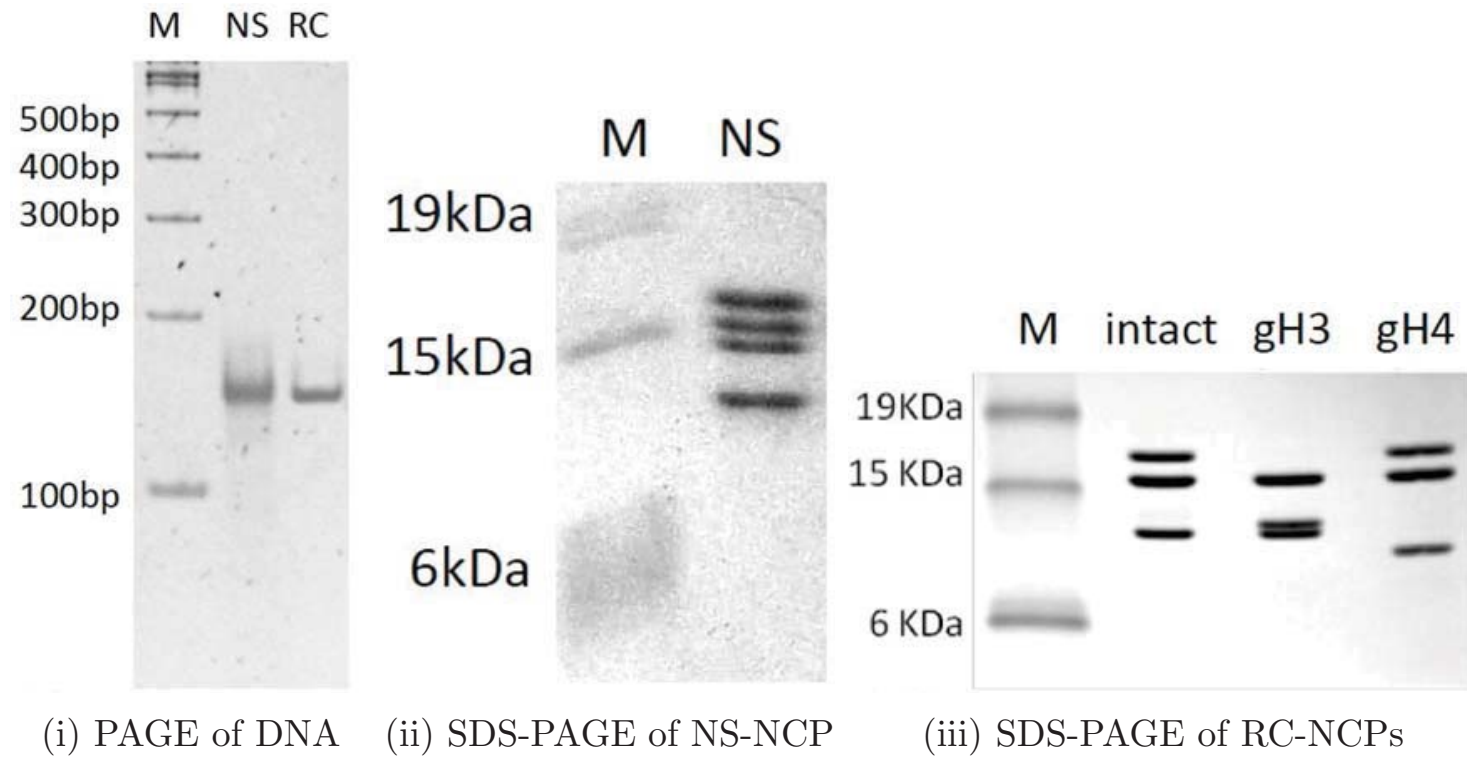

Figure 67. Characterization of Nucleosome Samples: (i) DNA extracted from NS-NCPs, "NS", and the recombinant 147 bp MMTV DNA, "RC", in 5\% PAGE. The "M" lane is the 100 bp DNA marker (Invitrogen). By analyzing the intensity distribution of each band, we obtained the full widths at half maximum (FWHM) of the bands as, 100 bp marker: 10 pixels, 200 bp marker: 7.5 pixels, NS-DNA: 15 pixels, RC-DNA: 10.5 pixels. Given the distance between $100 \mathrm{bp}$ and $200 \mathrm{bp}$ markers are $\sim 100$ pixels, the additional smearing of the NS-DNA band is estimated to be $\sim 10$ pixels in FWHM, corresponding to $\pm 5 \mathrm{bp}$. The maximum peak positions for NSDNA and RC-DNA are identical within experimental error, noting that the NS-DNA band intensity distribution is skewed toward longer DNA lengths as expected from the nucleosomal hindrance of Micrococcal nuclease digestion. We thus estimated the NS-DNA to be $147 \pm 5$ bp. (ii) SDS-PAGE (15\%) of natural-source NCP histones. The "M" lane is the protein marker (Invitrogen BenchMark ${ }^{\mathrm{TM}}$ ). (iii) SDS-PAGE of recombinant NCP histones ("intact" refers to the RC-NCP with all histone tails). Note that the recombinant $\mathrm{H} 2 \mathrm{~A}$ and $\mathrm{H} 2 \mathrm{~B}$ histones migrate as one band, while the $\mathrm{H} 2 \mathrm{~A}$ and $\mathrm{H} 2 \mathrm{~B}$ histones from NS-NCPs are well separated (ii). Possible explanations include sequence differences and post-translational modifications in NS-NCP histones only. 


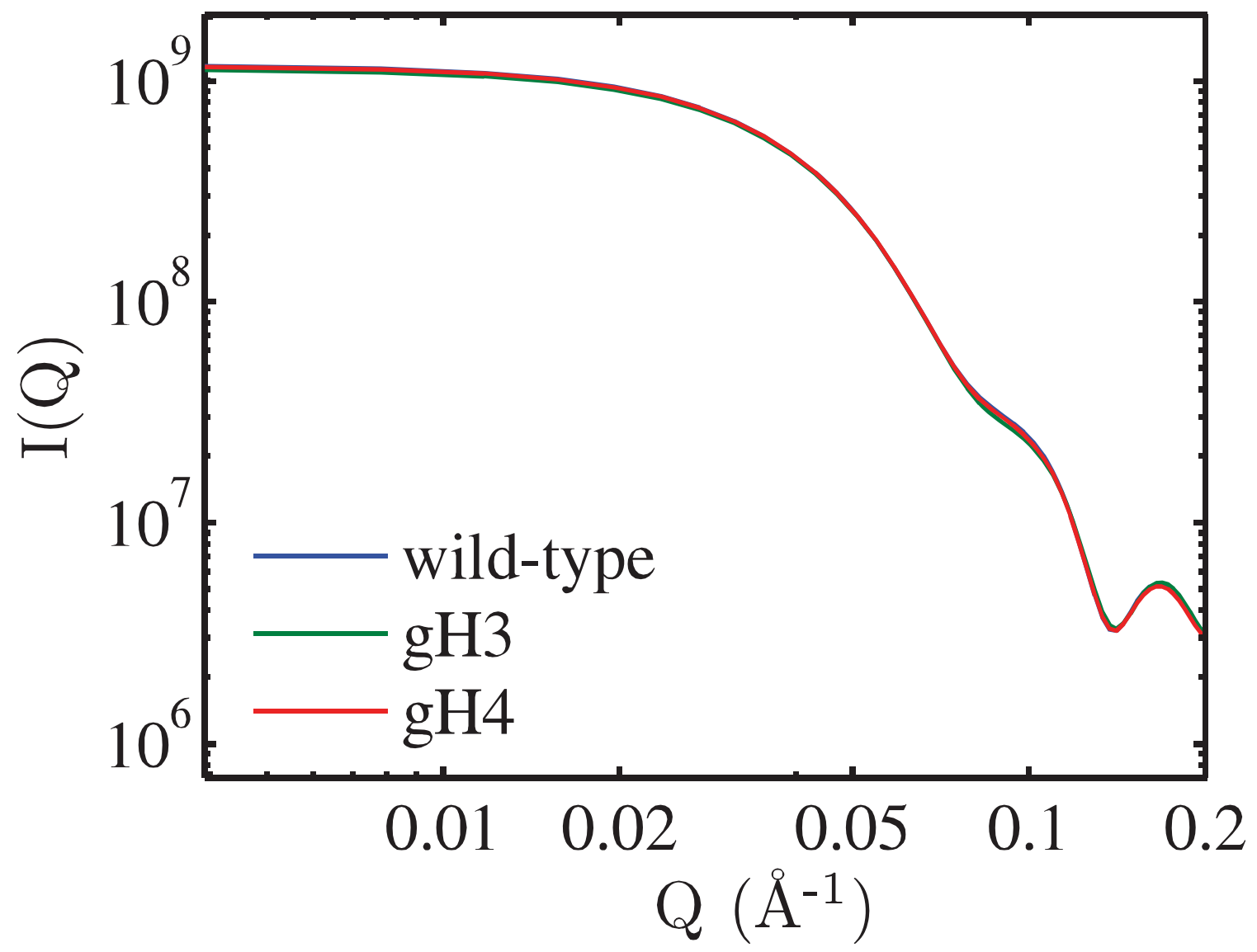

Figure 68. The Form Factors for the Nucleosome Constructs in this Study: The SAXS profiles are calculated based on the nucleosome crystal structure (1KX5) using Crysol [149]. Atomic structures for the histone-tail-deletion constructs (i.e., $\mathrm{gH} 3$ and $\mathrm{gH} 4 \mathrm{NCPs}$ ) are obtained by manual removal of the residues in accord with the constructs. The wild-type curve is used as the form factor for the NS-NCP and RC-NCP (results from the latter are shown in fig. 71). Comparisons of the three form factors indicate that tail deletions lead to rather small changes to the shape of the SAXS profile. 


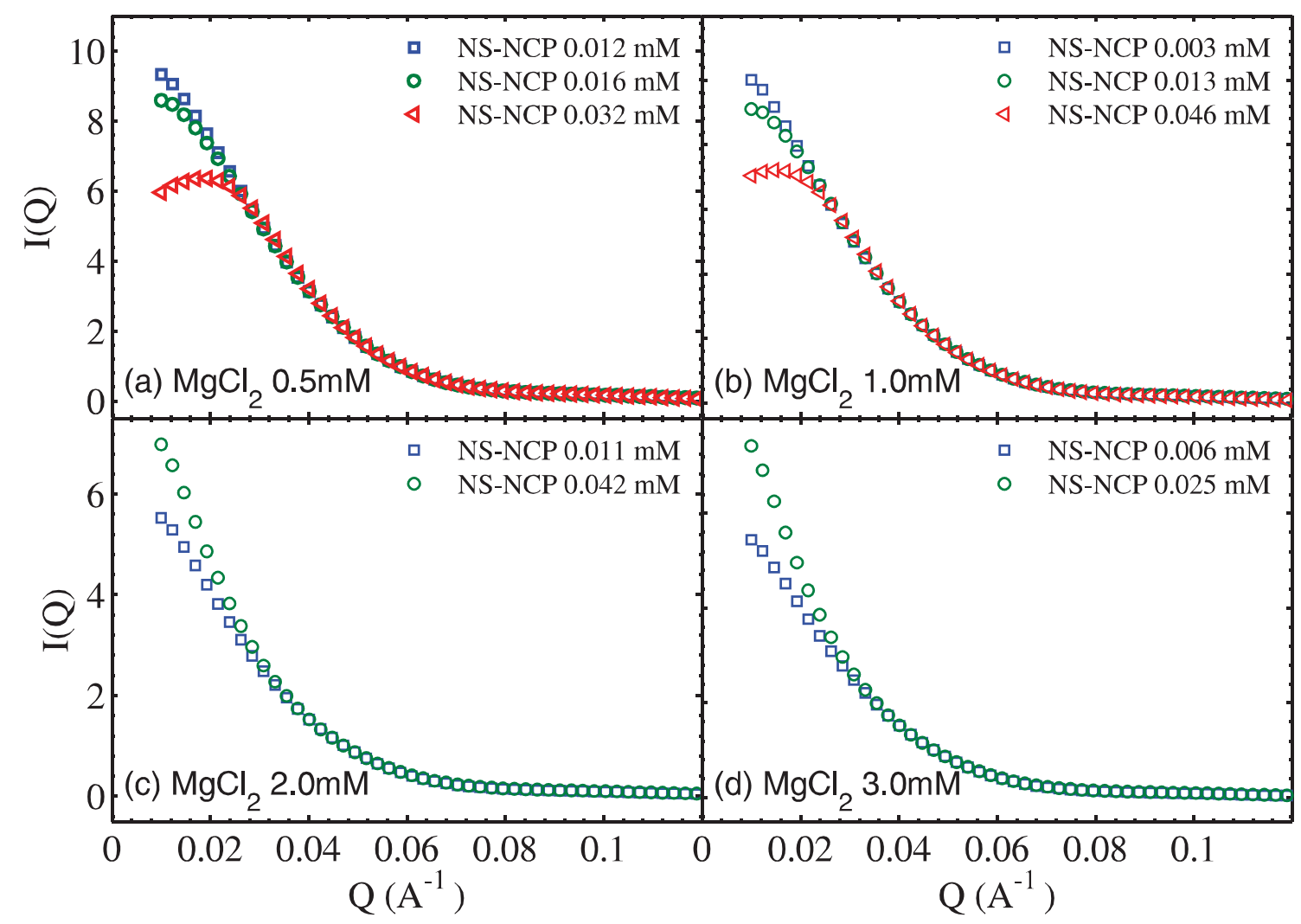

Figure 69. SAXS Profiles of NS-NCPs in a Series of $\mathbf{M g C l}_{2}$ Concentrations (no theoretical fits): Inter-NCP repulsion (low Q downturn) was observed at 0.5 and $1.0 \mathrm{mM} \mathrm{MgCl}_{2}$, while attraction (low Q upturn) were observed at 2.0 and $3.0 \mathrm{mM}$ $\mathrm{MgCl}_{2}$. We have not been able to obtain satisfactory fits to the full SAXS profiles in $\mathrm{Mg}^{2+}$ salts using the GOCM. We do not yet know the reason. One speculation is that the short-range inter-NCP interactions become strongly anisotropic in $\mathrm{Mg}^{2+}$ salts and the GOCM fails, or that there exist oligomeric states of NCPs in the presence of $\mathrm{Mg}^{2+}$. Still, the qualitative observations of inter-NCP interactions (i.e., repulsion versus attraction) hold. Quantitative modeling of the SAXS data in $\mathrm{Mg}^{2+}$ salts is being actively pursued in our groups. 


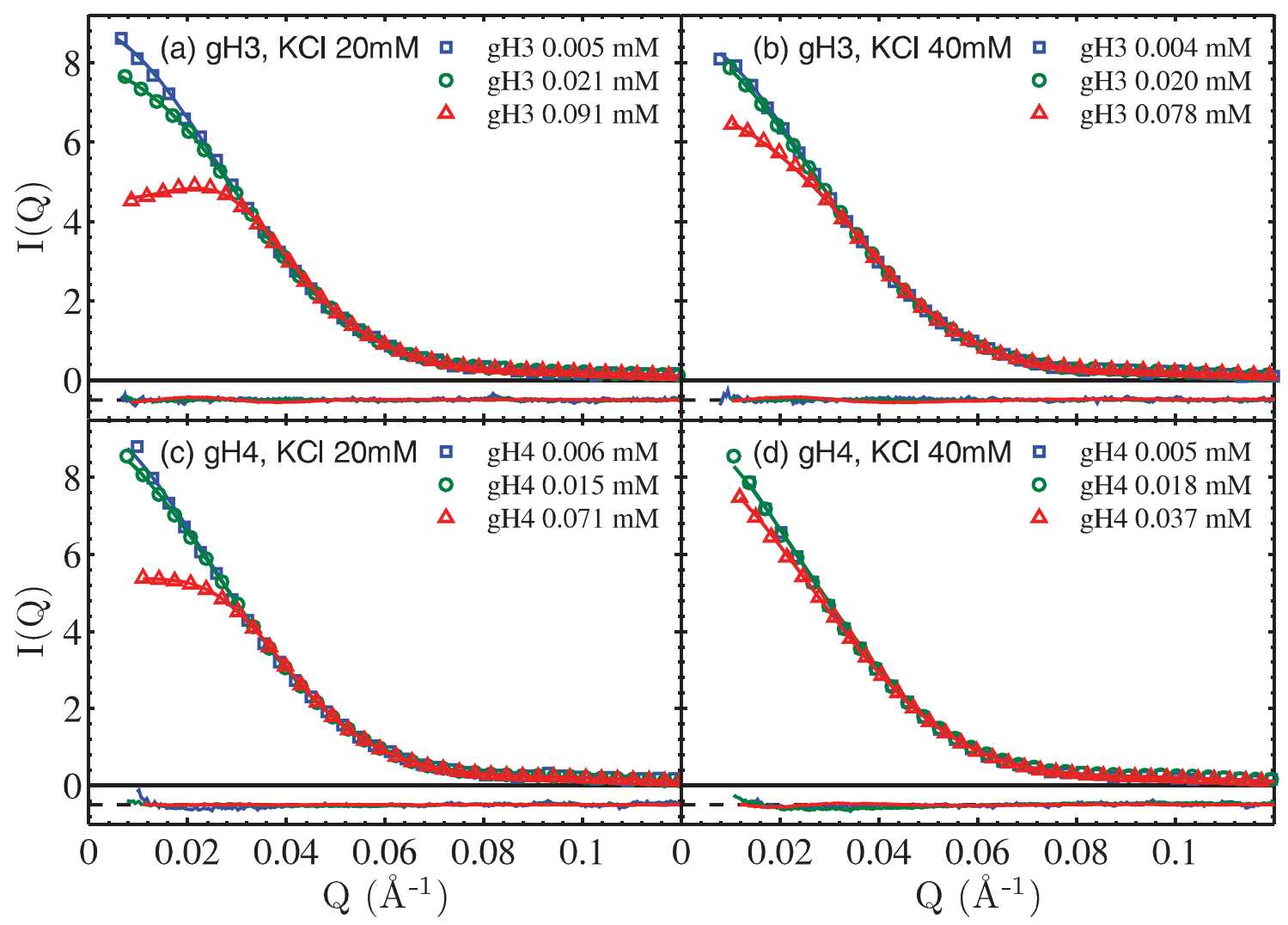

Figure 70. SAXS Profiles and GOCM Fits of gH3 and gH4 RC-NCPs in 20 and $40 \mathrm{mM} \mathrm{KCl:} \mathrm{I}(\mathrm{Q}) \mathrm{s}$ are normalized by $\mathrm{NCP}$ concentrations to assist visual comparison. Each panel shows experimental I(Q)s (symbols) at a series of $[\mathrm{NCP}] \mathrm{s}$ as indicated in the legends, together with their respective theoretical fits (lines). The residues are shown with an offset at the same scale. The pertinent pair-potential parameters are (discussed in detail in chapter 2), (a) $Z_{\text {eff }}=22(1)$ e, $\sigma_{N}=130 \AA$; (b) $Z_{\text {eff }}=21(1) e, \sigma_{N}=110 \AA$; (c) $Z_{\text {eff }}=23(1) e, \sigma_{N}=130 \AA$; (d) unreliable fitted values due to the relative low $[\mathrm{NCP}] \mathrm{s}$ and thus not given. 


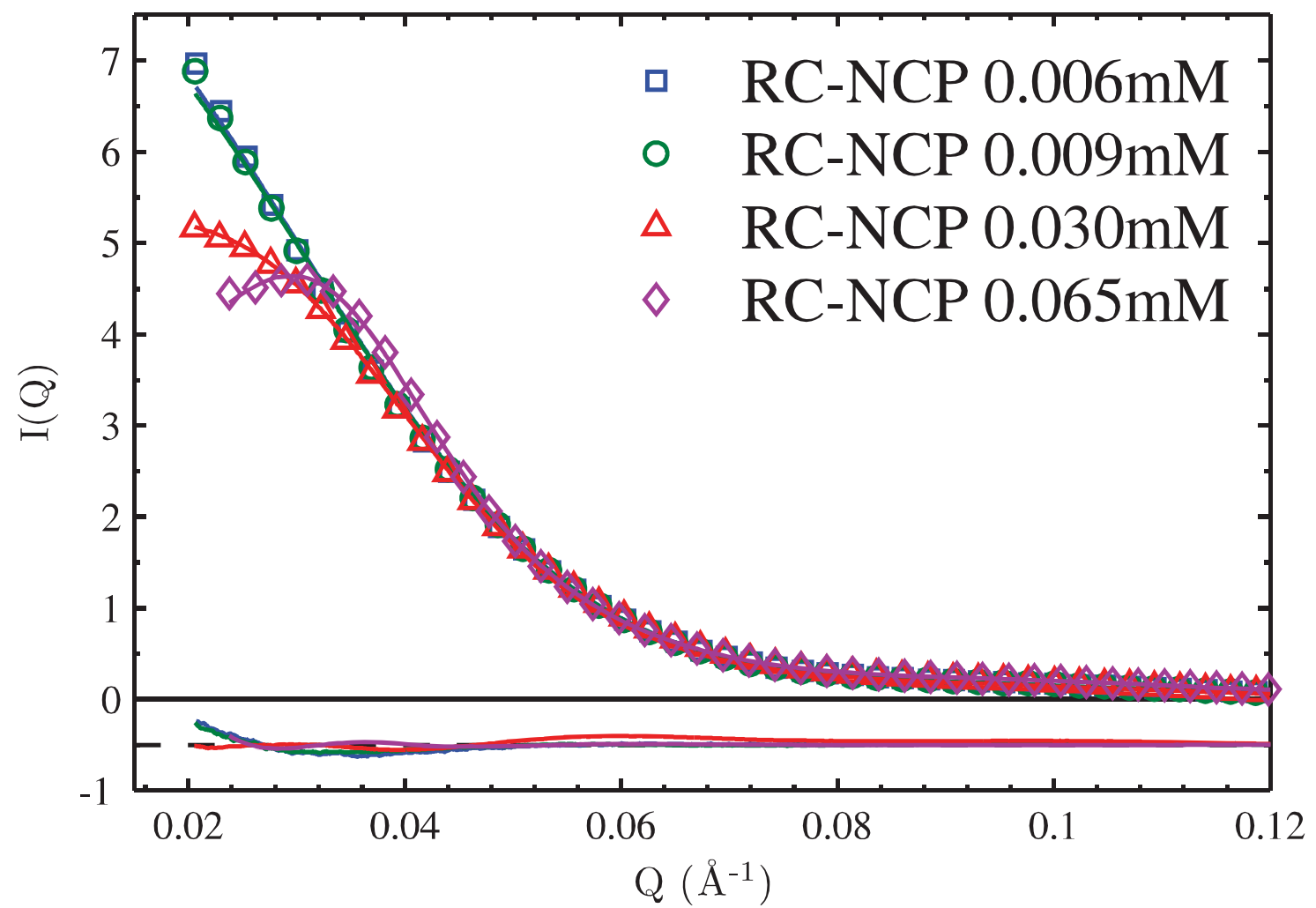

Figure 71. SAXS Profiles and GOCM fits of Intact Recombinant RC-NCPs in $10 \mathrm{mM} \mathrm{KCl}$ : Annotations follow that of fig. 70, namely: I(Q)s are normalized by NCP concentrations to assist visual comparison. Experimental I(Q)s (symbols) are shown at a series of $[\mathrm{NCP}] \mathrm{s}$ as indicated in the legend, together with their respective theoretical fits (lines). The residues are shown with an offset at the same scale. The pair-potential parameters are, $Z_{\mathrm{eff}}=21(1)$ e, $\sigma_{N}=140 \AA$. 


\section{Modeling the Structure Factor with a Two-DH Inter-NCP Interaction Potential}

In addition to the use of either repulsive or attractive DH potential to fit the structure factor as in the main text, the inclusion of both DH terms in the inter-NCP potential was presented here. This potential takes the form shown in below (the same as eq. (19) in Chapter 2),

$$
U(r)= \begin{cases}\infty & r<\sigma_{N} \\ \frac{Z_{\mathrm{eff}}^{2}}{\epsilon\left(1+\frac{\kappa_{D} \sigma_{N}}{2}\right)^{2}} \frac{e^{-\kappa_{D}\left(r-\sigma_{N}\right)}}{r}-\frac{Z_{\mathrm{attr}}^{2}}{\epsilon\left(1+\frac{\kappa_{\mathrm{attr}} \sigma_{N}}{2}\right)^{2}} \frac{e^{-\kappa_{\mathrm{attr}}\left(r-\sigma_{N}\right)}}{r} & r \geq \sigma_{N}\end{cases}
$$

Here the hard-core potential has a fixed diameter of $\sigma_{N}=100 \AA$ for all samples; the electrostatic repulsive DH term has Debye length $\left(1 / \kappa_{D}\right)$ given by the ionic strength;

and the attractive $\mathrm{DH}$ has decay length $\left(1 / \kappa_{\text {attr }}\right)$ fixed as $4.8 \AA$, equivalent to the Debye length at $400 \mathrm{mM}$ monovalent salt. It should be noted that, as we do not know the exact nature of the inter-NCP attraction, the choice of a DH potential for inter-NCP attraction is convenient due to the available computational methods [155] but somewhat arbitrary. The choice of $4.8 \AA$ Debye length is due to the most likely short-range nature of inter-NCP attraction, the observation of inter-molecular attractions of $4.8 \AA$ decay length between various types of molecular surfaces [156], and the necessity of significant differences from the Debye length of the repulsive DH term in salt conditions from 10 to $200 \mathrm{mM} \mathrm{KCl}$.

This leaves two fitting parameters in the inter-NCP potential, the effective charges of the repulsive $\left(Z_{\text {eff }}\right)$ and attractive $\left(Z_{\text {attr }}\right)$ DH terms. However, one complication is that there exists significant correlation between the two fitting parameters, particularly at high salts when their Debye lengths are close. This is because of the mutual 
cancellation effects of the two potentials. For this reason, while excellent fits were obtained (not shown), correlations between $Z_{\text {eff }}$ and $Z_{\text {attr }}$ values were evident from their fluctuations from sample to sample. To avoid such large fluctuations and facilitate comparisons between different salt conditions, we made one assumption that the attractive potential for each NCP construct (i.e., NS, gH3, or gH4 NCP) is independent of ion condition, i.e., the same $Z_{\text {attr }}$ used for all salt conditions. The $Z_{\text {attr }}$ value for each construct was determined by averaging the fitted $Z_{\text {attr }}$ values at different salts for the particular construct. This procedure led to decreases of the fitting qualities, though rather satisfactory agreements with experimental data were obtained for all samples.

We show the fitted results based on the procedure discussed above in figs. $72-$ 75 and the fitted potential parameters in table 2. Compared with the fits with one DH-term potential discussed in the main text, the decrease of fitting quality is more significant for the more concentrated NCP samples. As the attractive DH term is present at all salt conditions, the fitted repulsive effective charges, $Z_{\text {eff }}$, are larger than those obtained with the one-DH repulsive potential as expected. The effective charges $(<50 e)$ are still substantially smaller than the bare charge $(\sim 150 e)$ or the theoretical renormalized charges $(>100 e)$. Qualitative comparisons are thus unchanged, while the fitted inter-NCP potentials are changed quantitatively as given in table 2. For the attractive $\mathrm{DH}$ term, this fitting procedure obtains the effective charge $Z_{\text {attr }}$ of 200, 165, and $154 e$ for the NS, gH3, and gH4 NCPs respectively. This is consistent with the results from one-DH potential fits indicating decreased attraction with tail deletions.

Overall, the inclusion of a persisting attractive term is intellectually pleasing as inter-NCP attraction (e.g., mediated by histone tails) is very likely present at all times, though overwhelmed by electrostatic repulsion at low salts. However, simultaneous determinations of both repulsive and attractive contributions are challenging. 


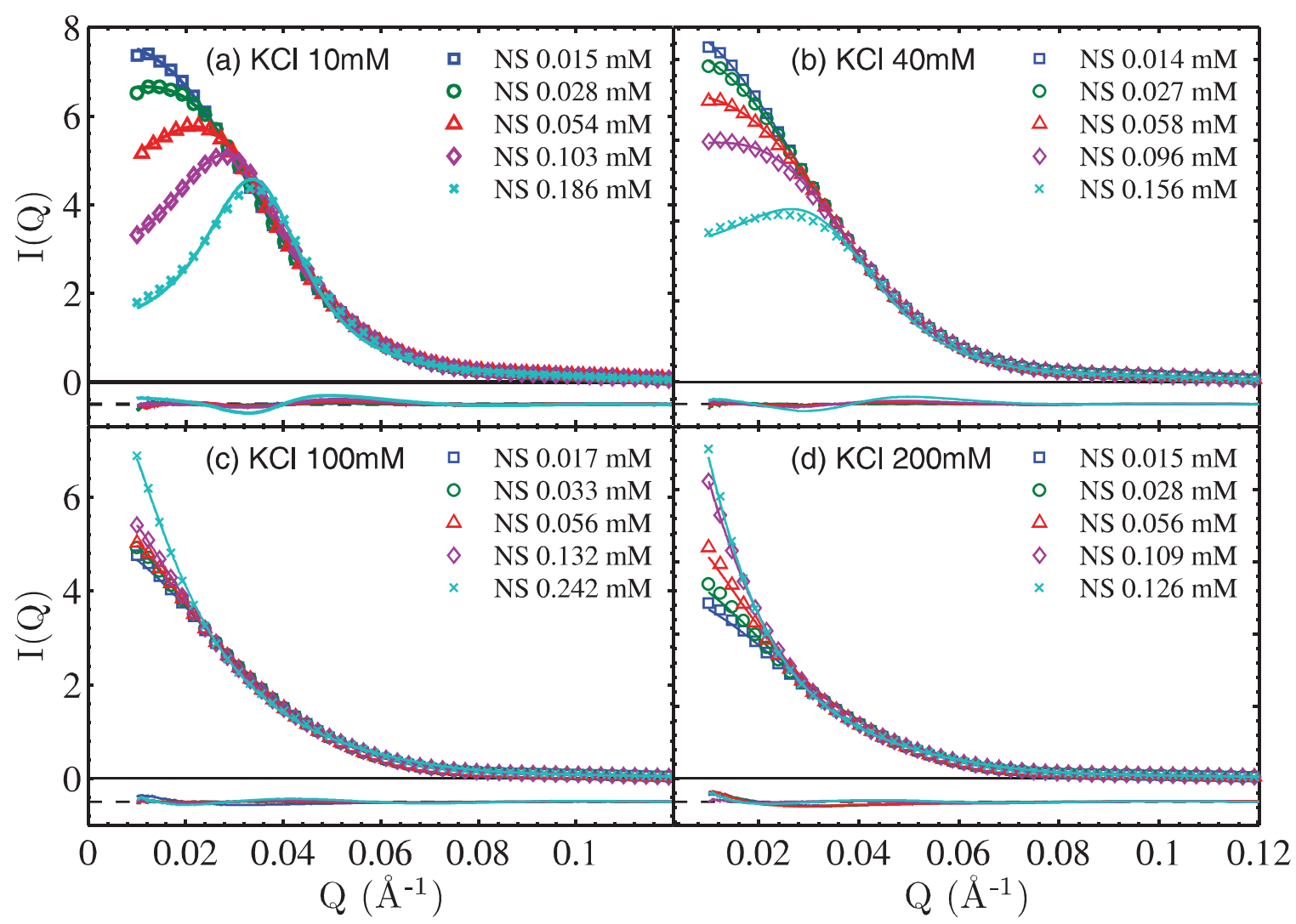

Figure 72. SAXS profiles of NS-NCPs in a Series of $\mathrm{KCl}$ concentrations with fits based on the two-DH inter-NCP potential: Annotations are the same as in fig. 9 in the main text, namely: $\mathrm{I}(\mathrm{Q}) \mathrm{s}$ are normalized by $\mathrm{NCP}$ concentrations to assist comparison. Each panel shows experimental $\mathrm{I}(\mathrm{Q}) \mathrm{s}$ (symbols) at a series of $[\mathrm{NCP}] \mathrm{s}(0.1 \mathrm{mM}$ gives $\sim 20 \mathrm{mg} / \mathrm{mL})$ as indicated in the legends, together with their respective theoretical fits (lines). The residues are shown with an offset at the same scale. 


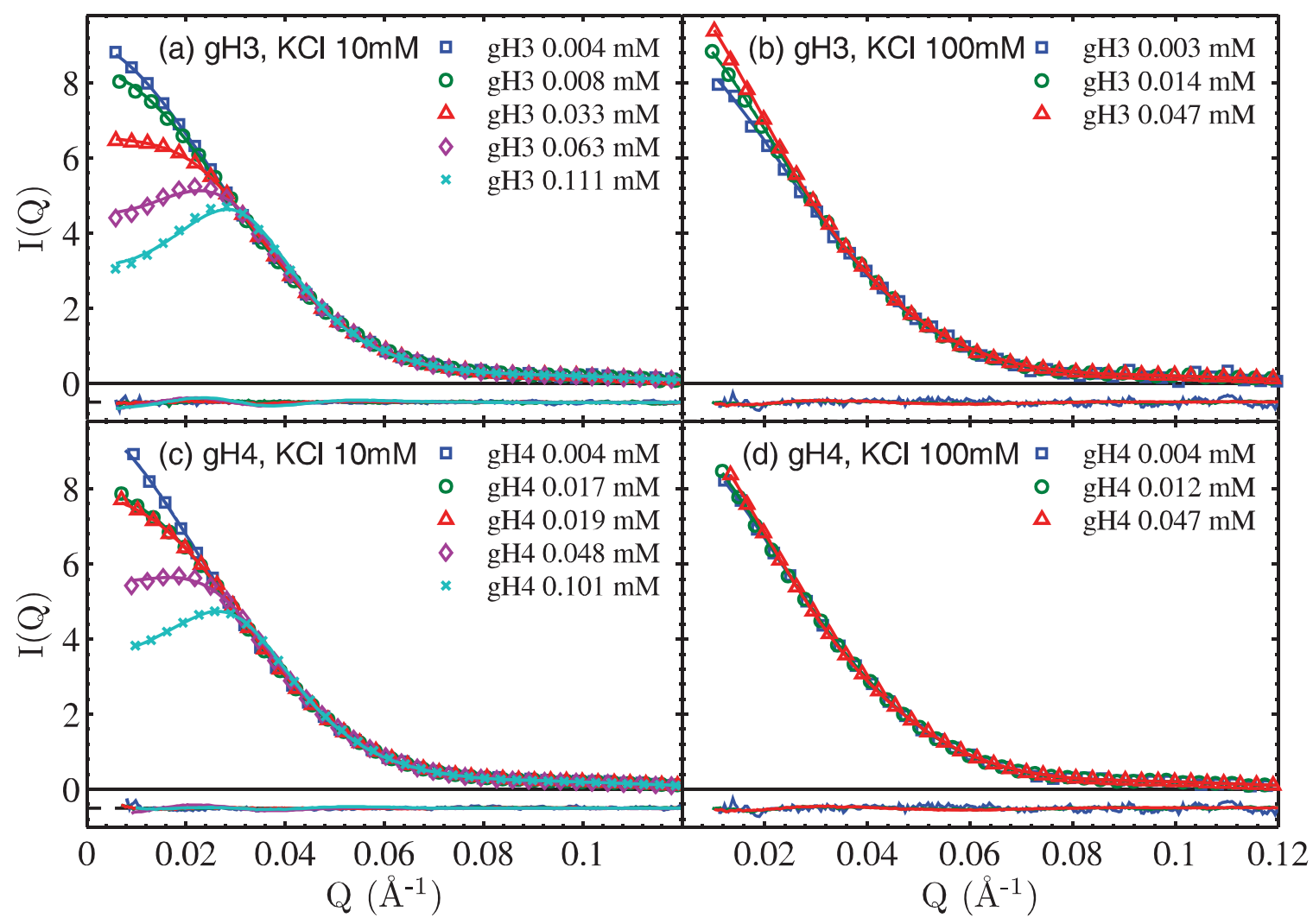

Figure 73. SAXS profiles of gH3 and $\mathrm{gH} 4 \mathrm{NCPs}$ in 10 and $100 \mathrm{mM} \mathrm{KCl}$ with fits based on the two-DH inter-NCP potential: Annotations follow that of fig. 9 in the main text, namely: $\mathrm{I}(\mathrm{Q}) \mathrm{s}$ are normalized by $\mathrm{NCP}$ concentrations to assist comparison. Each panel shows experimental $\mathrm{I}(\mathrm{Q}) \mathrm{s}($ symbols) at a series of $[\mathrm{NCP}] \mathrm{s}(0.1 \mathrm{mM}$ gives $\sim 20 \mathrm{mg} / \mathrm{mL})$ as indicated in the legends, together with their respective theoretical fits (lines). The residues are shown with an offset at the same scale. 


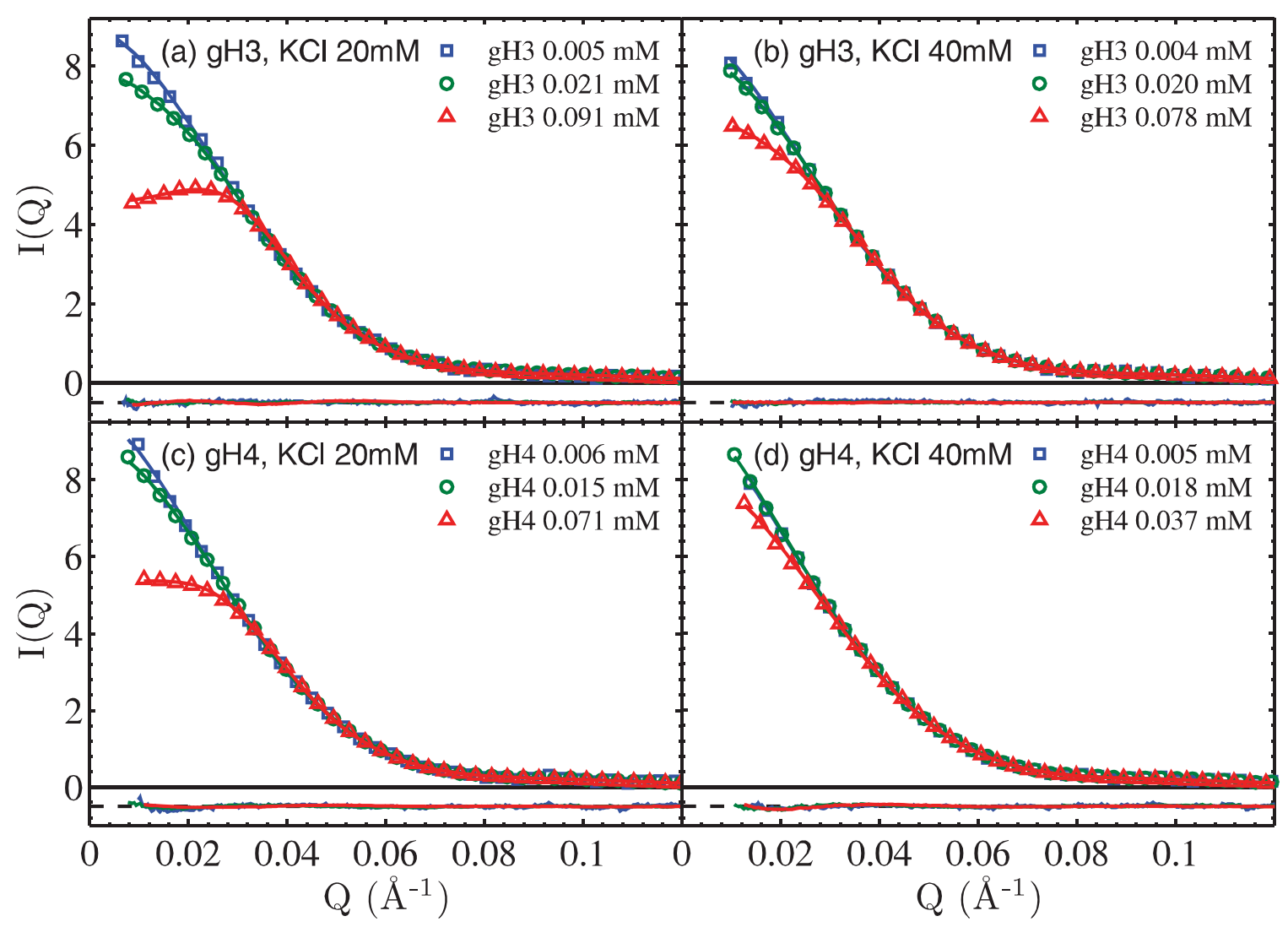

Figure 74. SAXS profiles of gH3 and gH4 NCPs in 20 and $40 \mathrm{mM} \mathrm{KCl}$ with fits based on the two-DH inter-NCP potential: Annotations follow that of fig. 9 in the main text, namely: $\mathrm{I}(\mathrm{Q}) \mathrm{s}$ are normalized by $\mathrm{NCP}$ concentrations to assist comparison. Each panel shows experimental $\mathrm{I}(\mathrm{Q}) \mathrm{s}$ (symbols) at a series of $[\mathrm{NCP}] \mathrm{s}(0.1 \mathrm{mM}$ gives $\sim 20 \mathrm{mg} / \mathrm{mL})$ as indicated in the legends, together with their respective theoretical fits (lines). The residues are shown with an offset at the same scale. 


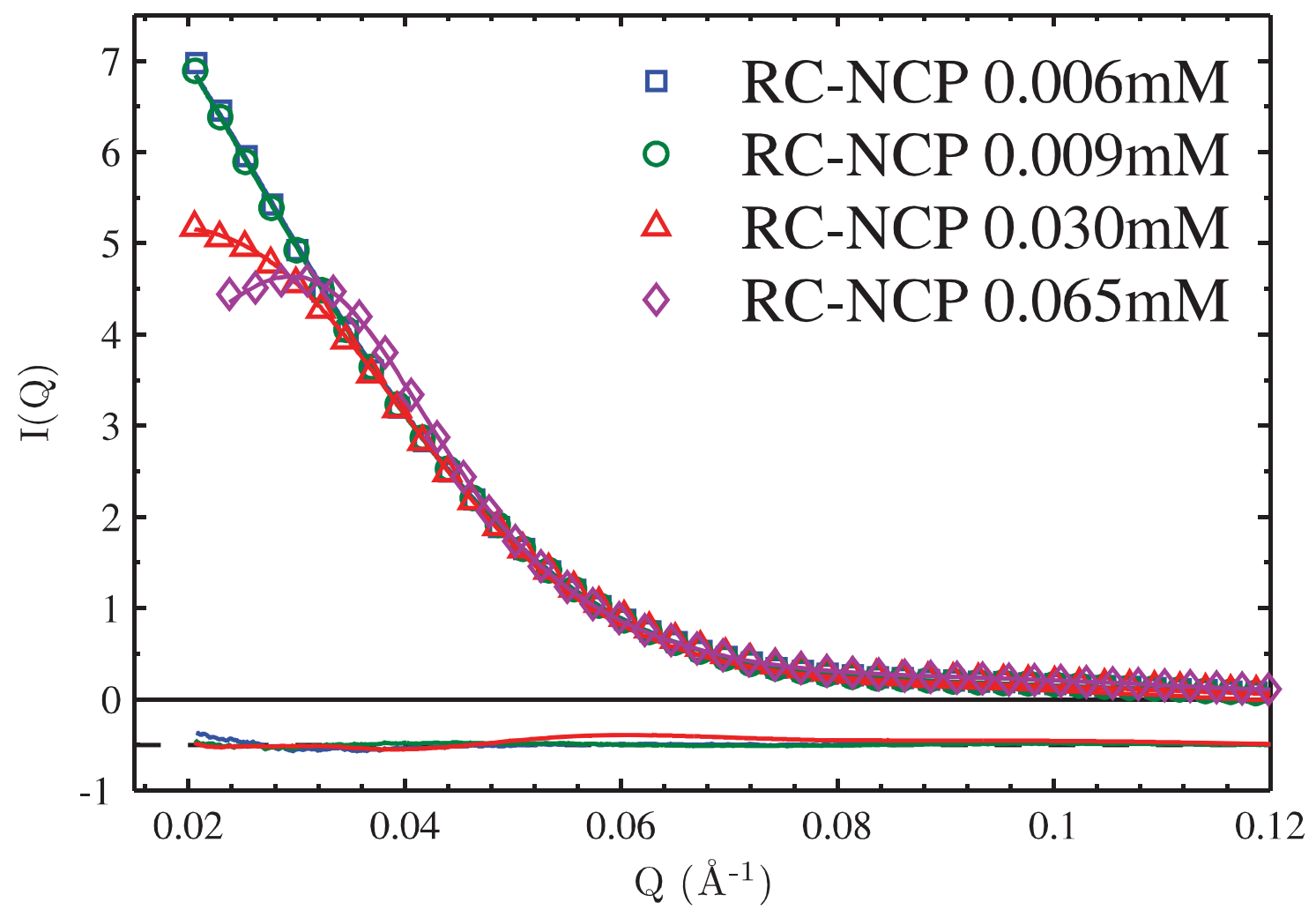

Figure 75. SAXS profiles of RC-NCPs in $10 \mathrm{mM} \mathrm{KCl}$ with fits based on the two-DH inter-NCP potential: Annotations follow that of fig. 9 in the main text, namely: I(Q)s are normalized by NCP concentrations to assist comparison. I(Q)s (symbols) are shown at a series of $[\mathrm{NCP}] \mathrm{s}(0.1 \mathrm{mM}$ gives $\sim 20 \mathrm{mg} / \mathrm{mL})$ as indicated in the legend, together with their respective theoretical fits (lines). The residues are shown with an offset at the same scale. The pertinent pair-potential parameters (discussed in the main text) are shown in table 2. 


\section{B: Supplemental Material for SAXS Experiments of Nucleo-}

\section{some Arrays}

\section{Concentration Adjustment}

Coherent scattering scales linearly in proportion to the amount of mass in the sample solution. Consequently, one can determine the sample concentration by scaling $I(Q)$ to the scattering from a know sample concentration. Figure 76 shows sample results from this concentration refining process.

In this example, we compare the identified a factor of two discrepancy between the experimental concentration measured using UV spectrophotometry and the scattering sample. This factor of two difference is likely a calculation error resulting from only reporting the concentration of DNA in solution, equal to half the nucleosome concentration.

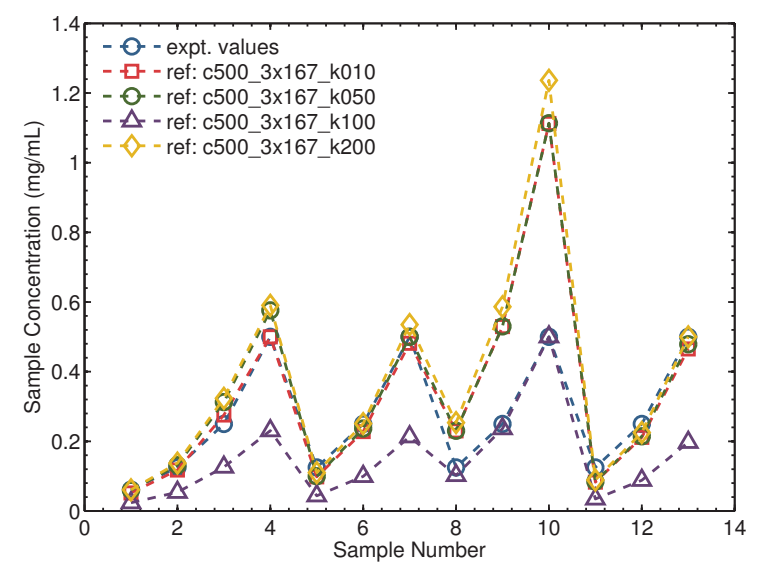

(i) Before Refinement

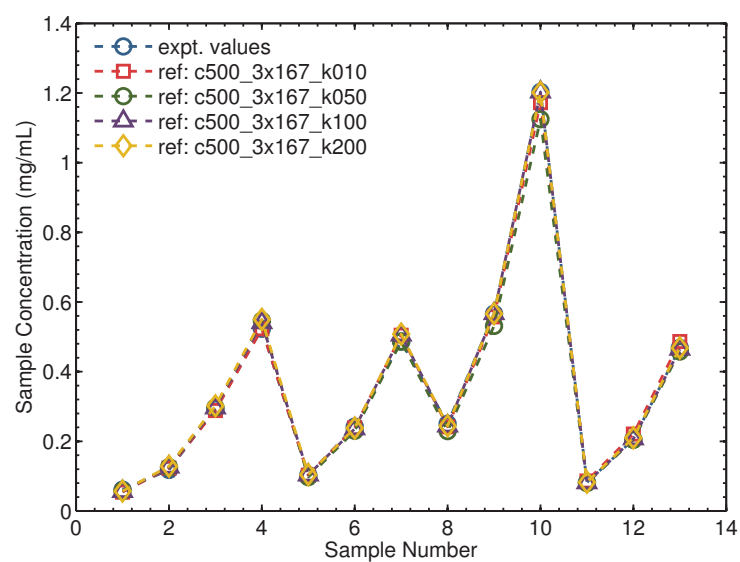

(ii) After Refinement

Figure 76. Sample Concentration Refinement: Example comparison between the expected concentration (expt. values) and the concentration calculated by scaling each $I(Q)$ to match the that of different reference (ref). This comparison identifies both minor deviations caused by the liquid sample cell and an approximate $2 \times$ difference caused by mistaking the DNA concentration for the total nucleosome concentration. When samples 1 (c500_3x167_k010), 5 (c500_3x167_k050), and 11 (c500_3x167_k200) are used as the reference, the scaling of samples 8-10 indicates the concentration to be between approximately double the expected concentration. 


\section{SAXS Results from $12 \times 167$ Arrays}
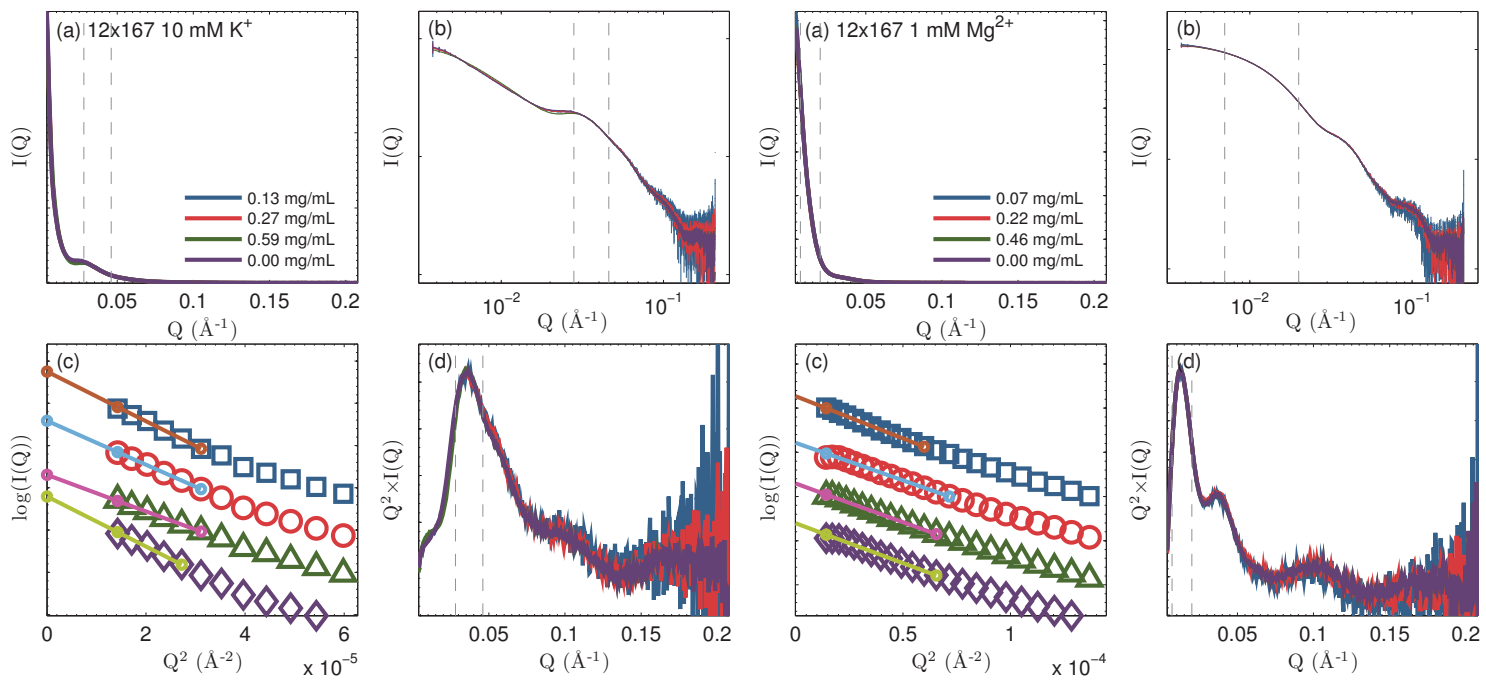

$10 \mathrm{mM} \mathrm{K}^{+}$
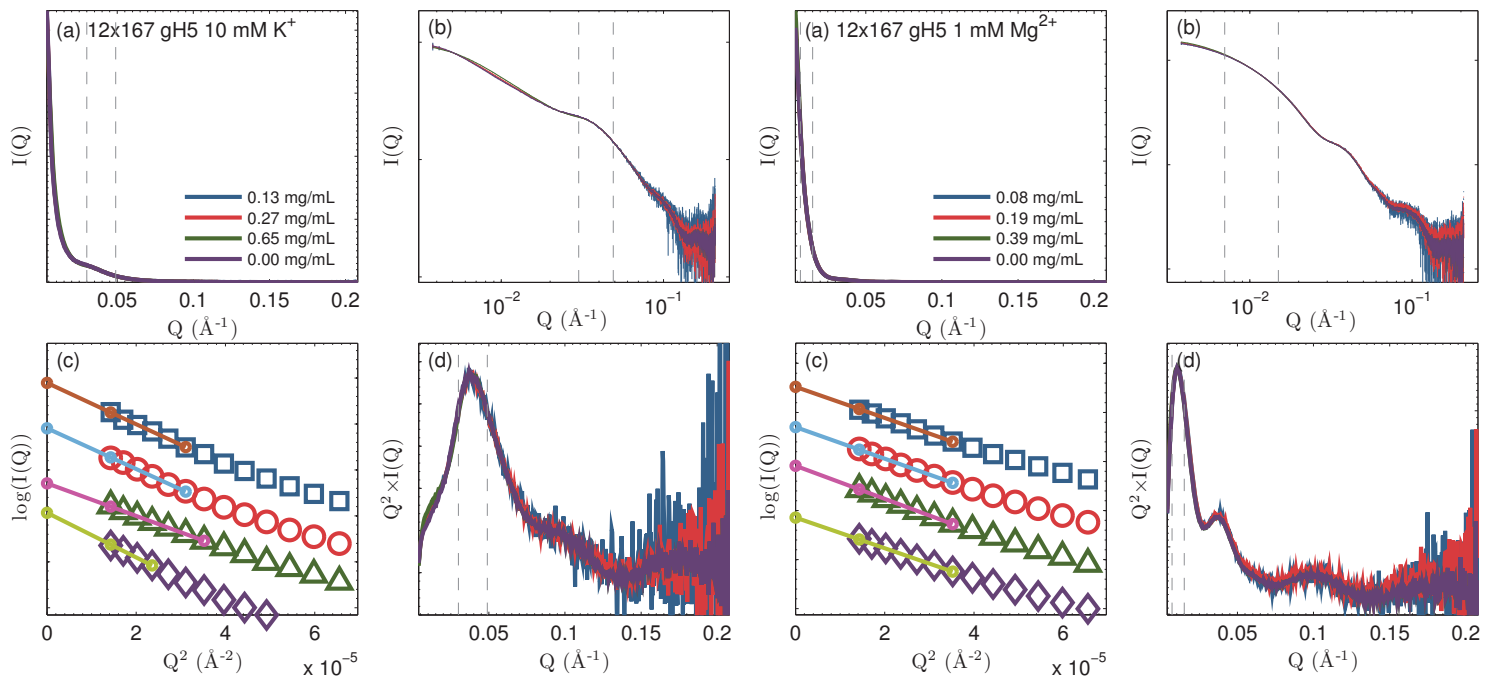

$100 \mathrm{mM} \mathrm{K}^{+}$

$1 \mathrm{mM} \mathrm{Mg}^{2+}$

Figure 77. Reduced SAXS Profiles for $12 \times 167$ Nucleosome Arrays: (a)-(b) $I(Q)$ plots respectively on a linear and logarithmic scale. (c) Guinier plot with $R_{g}$ fits from the lowest usable $Q$-point up to $Q_{\max }=1.3 / R_{g}$. (d) Kratky plot, emphasizing the low- $Q$ features. The dashed vertical lines indicate the $Q$-range used for scaling the data. 

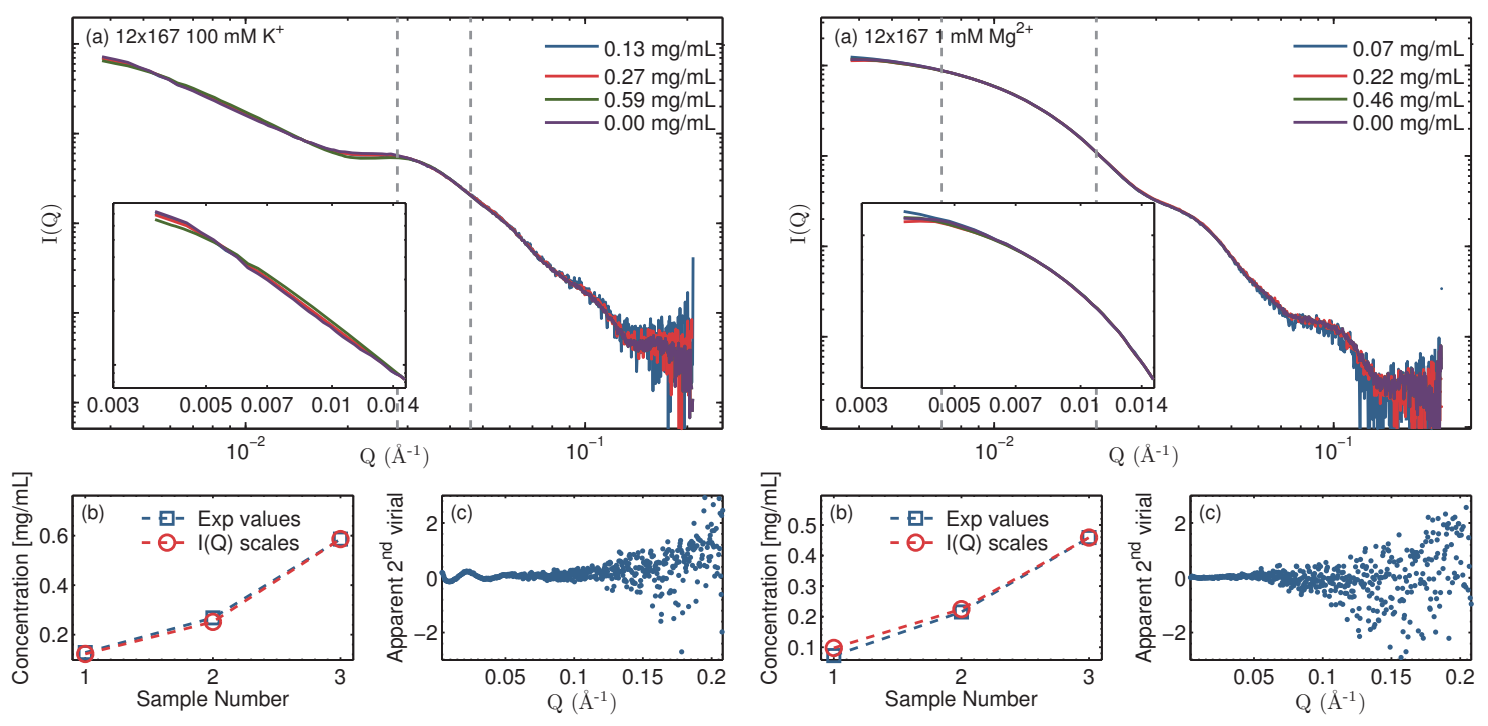

$10 \mathrm{mM} \mathrm{K}^{+}$

$50 \mathrm{mM} \mathrm{K}^{+}$

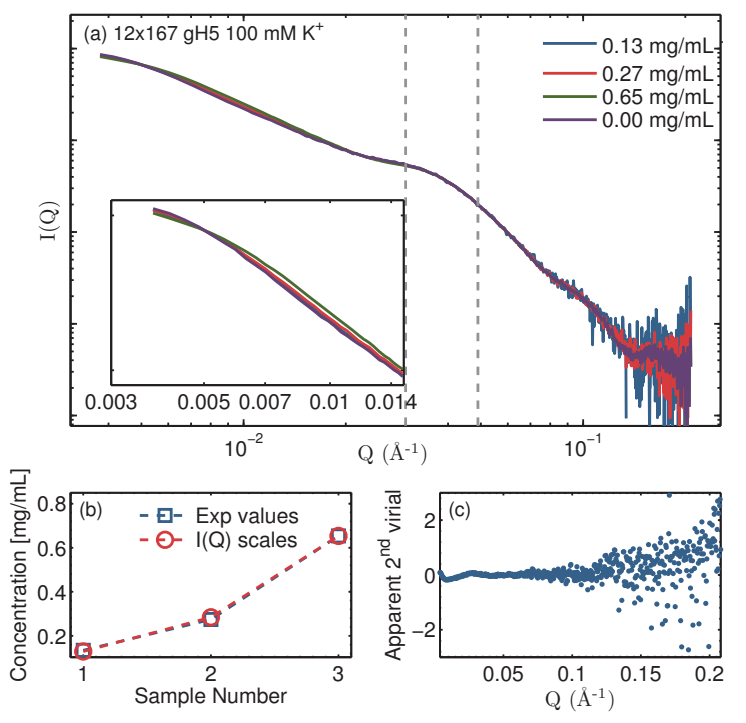

$100 \mathrm{mM} \mathrm{K}^{+}$
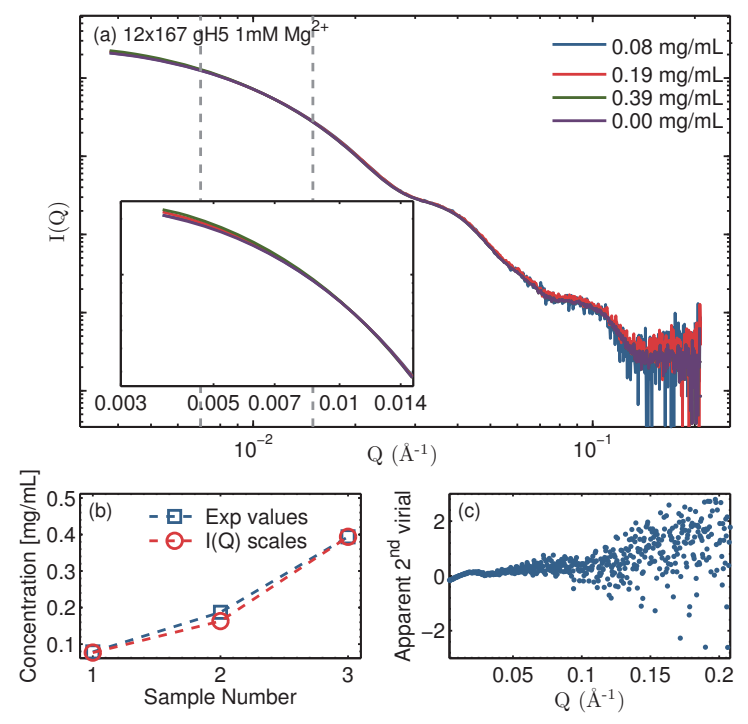

$1 \mathrm{mM} \mathrm{Mg}^{2+}$

Figure 78. Zero Concentration Extrapolation for $12 \times 167$ Nucleosome Arrays: (a)-(c) as in figs. 17-20, namely: (a) the reduced scattering intensity with an inset zoomed in on the lowest $Q$-range, both plotted using logarithmic scales. The vertical lines indicate the $Q$-range used for scaling the data before performing the extrapolation. (b) comparison between the prepared concentration and the relative concentration determined by the scale factor used in (a). (c) the apparent second virial coefficient. 


\section{C: CG MC Simulation Algorithm for ds-DNA Supplementary Material}

Figures 79-85 show the dihedral angle results from the $75 \mathrm{~ns}$ MD simulation of a solvated and ionized 12 bp B-form DNA model (PDB ID: 119D [137]). We calculated the seven dihedral angles for the ten central bp of the model (excluding the first and last bp), every $0.01 \mathrm{~ns}$. To prevent wraparound, the y-axis in each plot is offset so the median angle is zero and each dot represents the deviation from that median angle. The dark and light gray dots respectively represent DNA bases with BI and BII conformations (BI: $\varepsilon-\zeta>0$ and BII: $\varepsilon-\zeta>0$ ). The horizontal dashed lines indicate 2 standard deviations from the mean dihedral angle for all the bases (red), the BI bases (teal), and the BII bases (yellow). For the $\chi$ angle we separate the BI bases into purines (teal) and pyrimidines (royal blue) for direct comparison with the survey of crystal structures performed by Schneider et al. [136]. The solid lines show the corresponding rolling average calculated at each MD time step. The mean values and standard deviations are reported in the legends.

Table 3 summarizes these results in comparison with those from the survey of 34 B-form DNA crystal structures [136]. The mean angles obtained from this MD simulation match reasonably well to those from the crystal structures; the distribution of angles in broader for the MD results. 


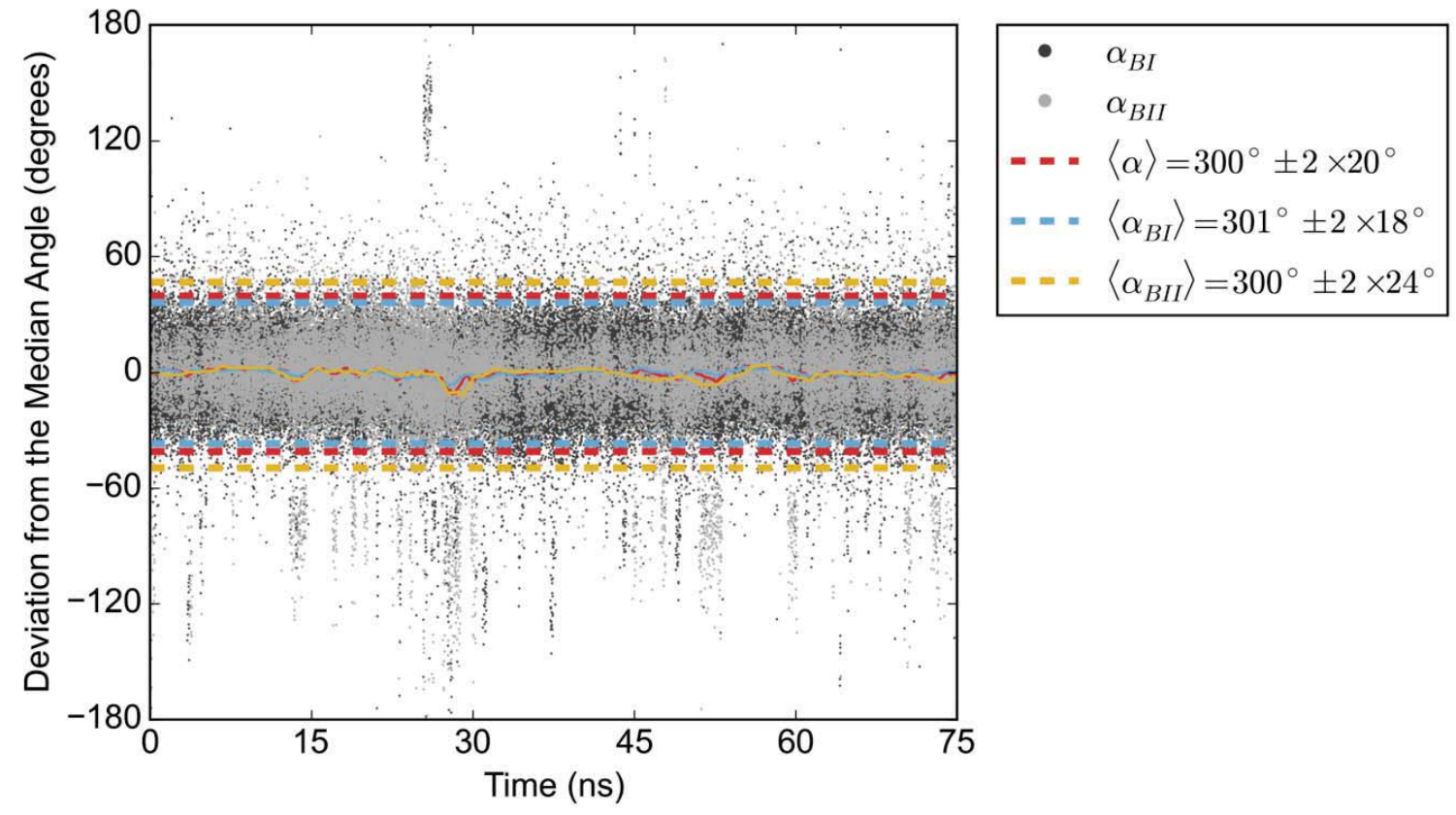

Figure 79. DNA Dihedral Angle $\alpha$ from the 75 ns MD simulation

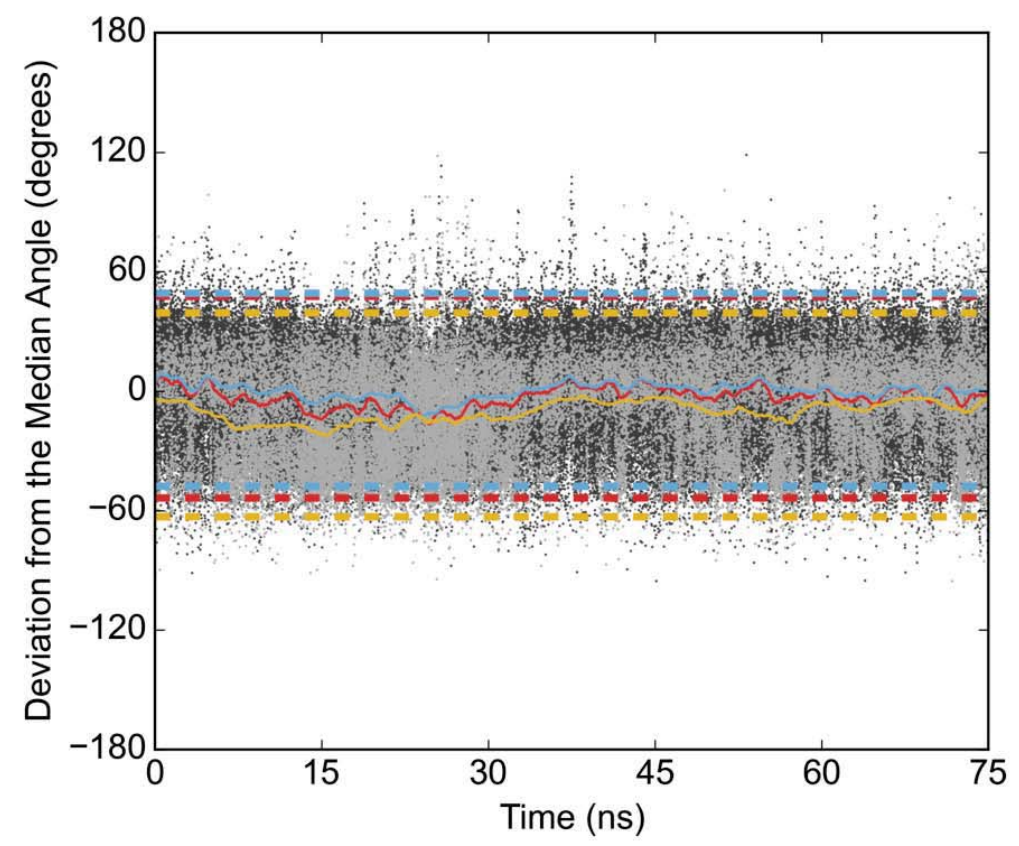

$$
\begin{array}{|cl|}
\hline & \beta_{B I} \\
- & \beta_{B I I} \\
=-- & \langle\beta\rangle=161^{\circ} \pm 2 \times 25^{\circ} \\
=-- & \left\langle\beta_{B I}\right\rangle=165^{\circ} \pm 2 \times 24^{\circ} \\
=- & \left\langle\beta_{B I I}\right\rangle=152^{\circ} \pm 2 \times 26^{\circ}
\end{array}
$$

Figure 80. DNA Dihedral Angle $\beta$ from the 75 ns MD simulation 


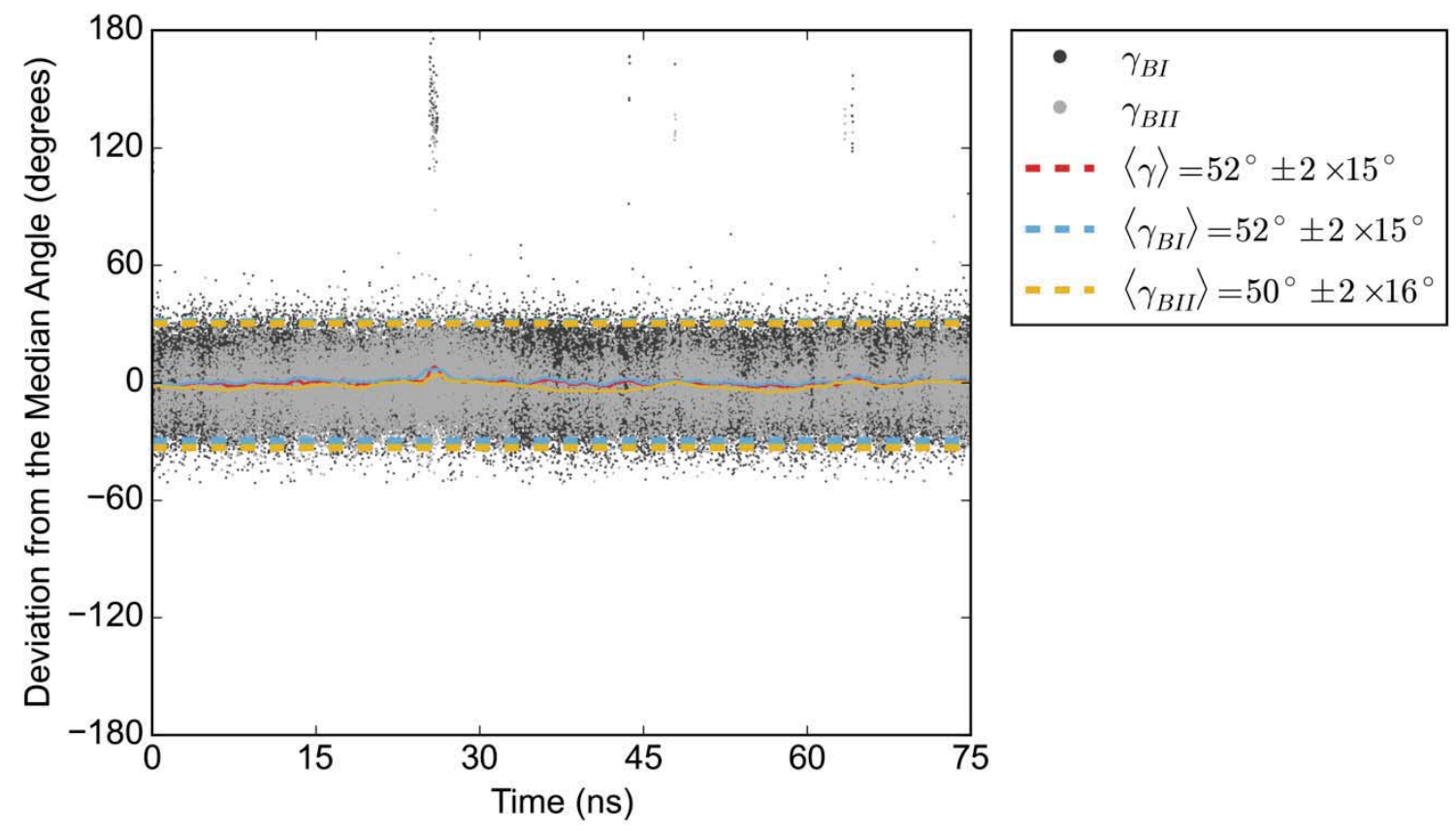

Figure 81. DNA Dihedral Angle $\gamma$ from the $75 \mathrm{~ns}$ MD simulation

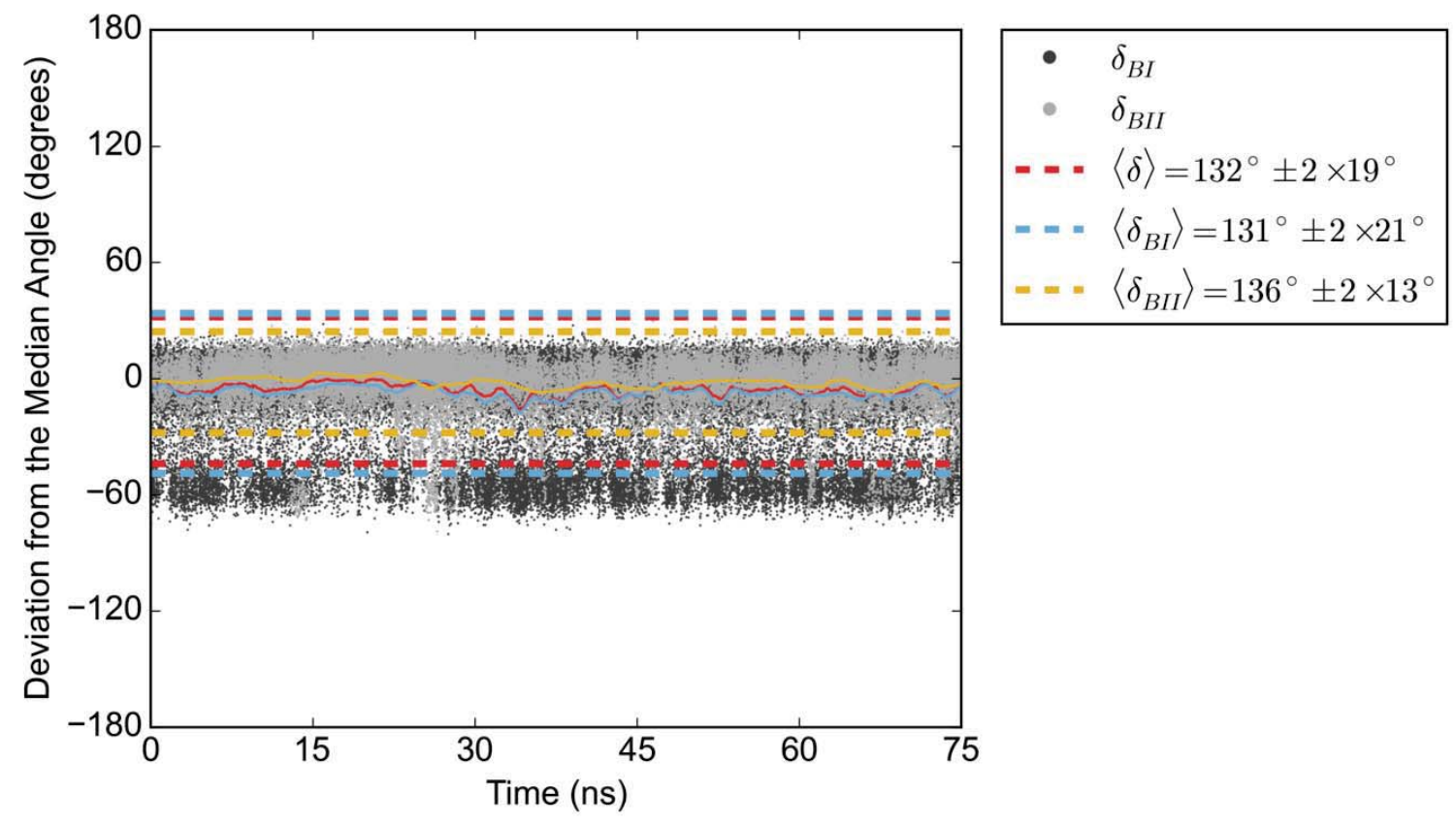

Figure 82. DNA Dihedral Angle $\delta$ from the $75 \mathrm{~ns}$ MD simulation 

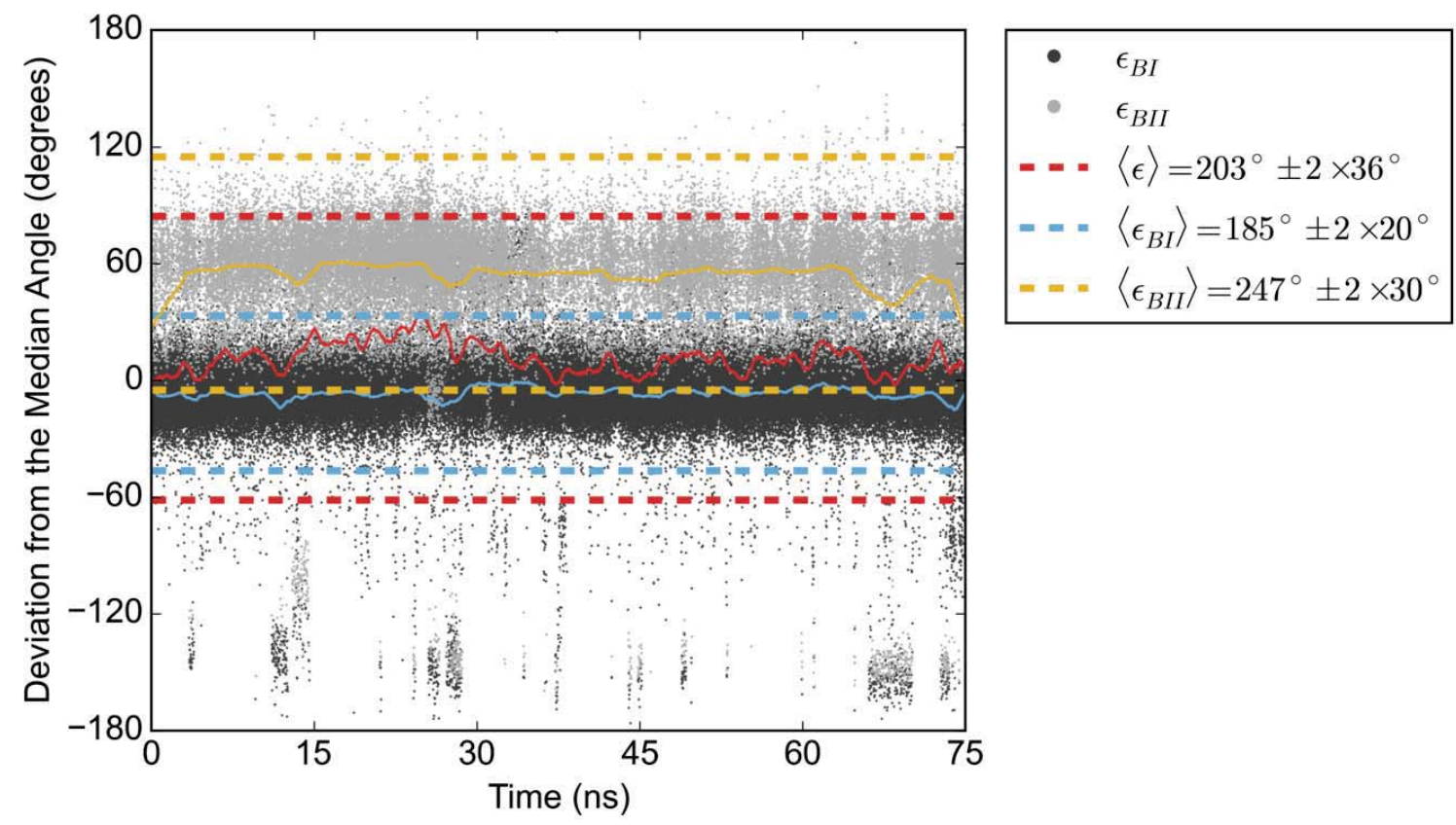

Figure 83. DNA Dihedral Angle $\varepsilon$ from the $75 \mathrm{~ns}$ MD simulation
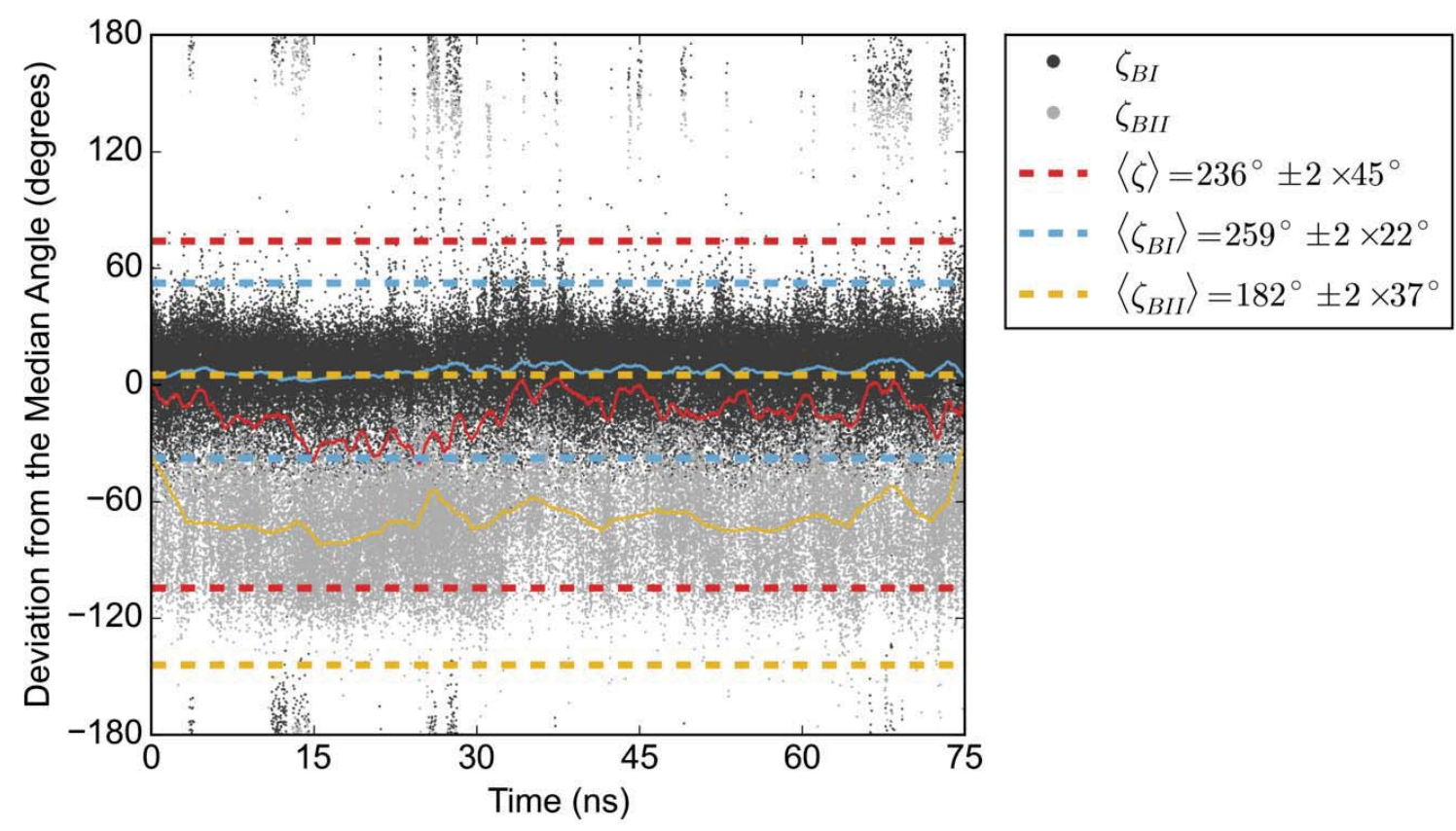

Figure 84. DNA Dihedral Angle $\zeta$ from the 75 ns MD simulation 


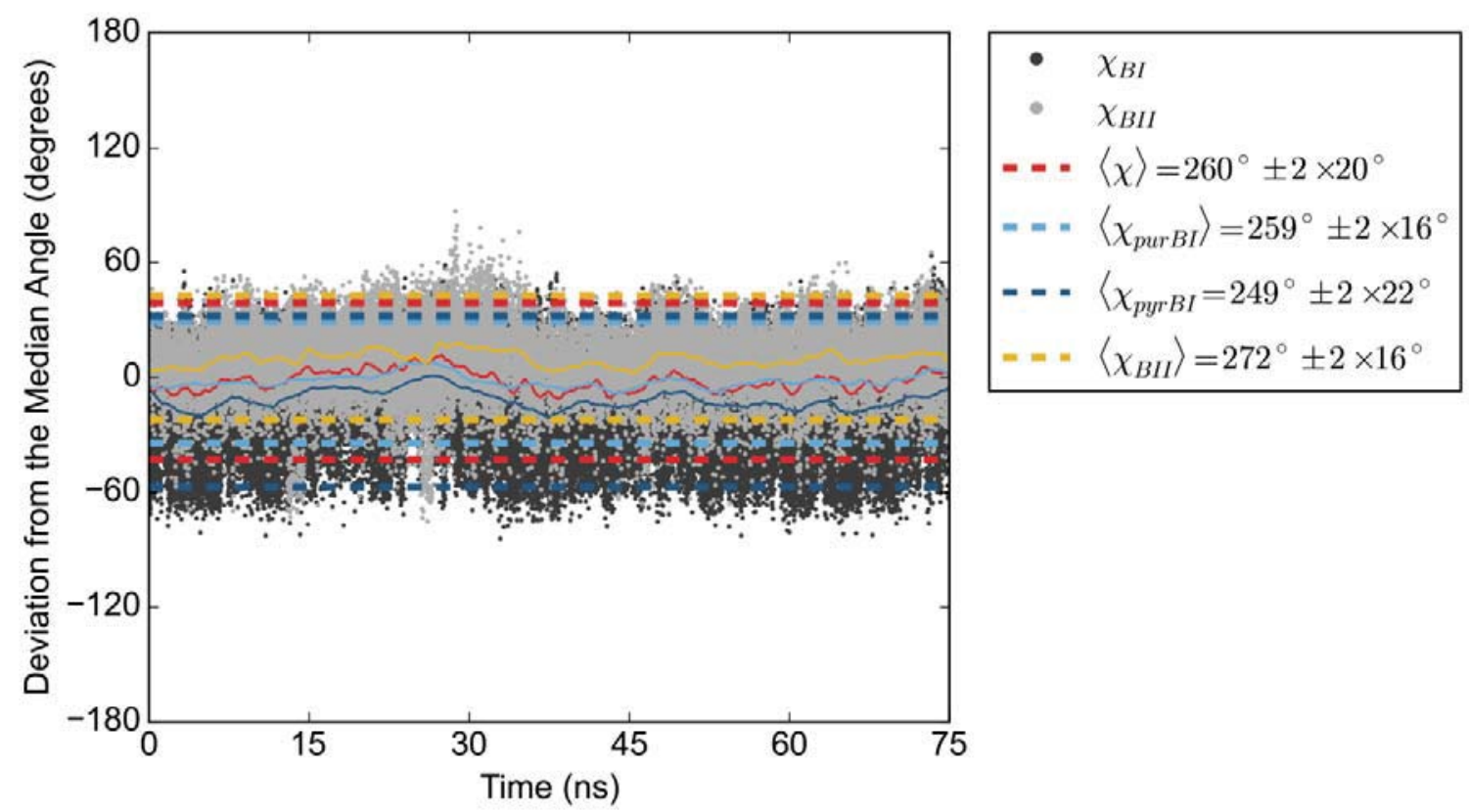

Figure 85. DNA Dihedral Angle $\chi$ from the $75 \mathrm{~ns}$ MD simulation 


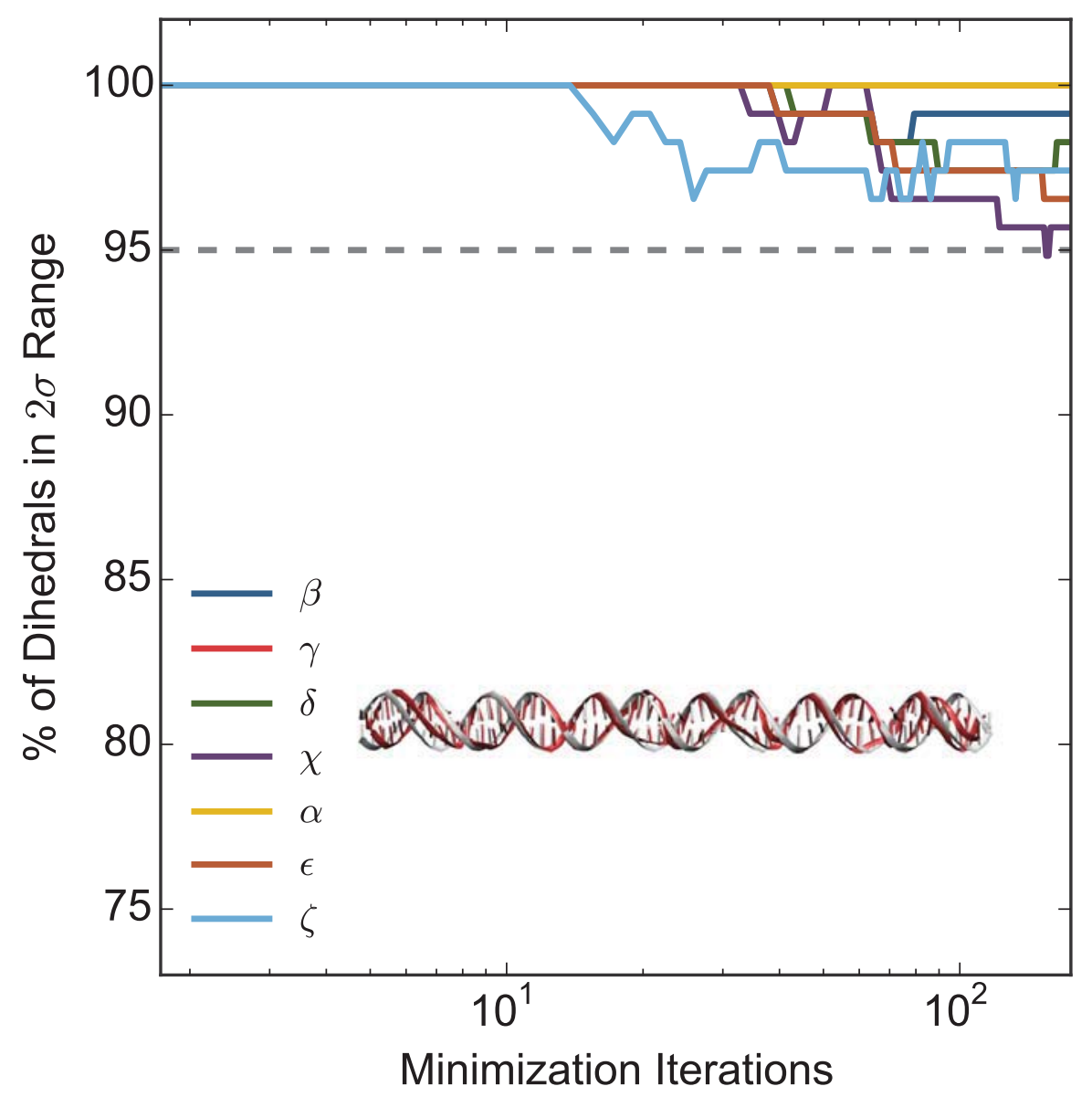

Figure 86. Iterative Minimization of dsDNA: Percent of DNA dihedral angles that are within 2 standard deviations of the mean dihedral angles calculated in the $75 \mathrm{~ns}$ MD simulation of dsDNA. This percentage is plotted as a function of the number of iterations of a 2000 step minimization procedure using CHARMM36 [138] force field. The dashed line shows the $95 \%$ cutoff for the percent of dihedral angles that should be within 2 standard deviations of the mean for a normal distribution. The inset DNA illustrates the DNA structure before minimization (white) and after 10 minimization iterations or 20000 total minimization steps (red). The dihedral angles for these structures do not exceed the normal range until around 70 repeated cycles, 140000 minimization steps, but the illustration shows that after as few as 10 minimization cycles, when almost 100 percent of each of dihedral angles are within their acceptable ranges, the DNA has experienced significant deformation of the major and minor groove. Additional minimization cycles only compounds this deformation of the DNA structure. Consequently, we recommend performing a single iteration of 2000 minimization steps after completing all MC simulations. Figure 51 shows that this is sufficient to relax the strain between the O3' atom of one base and the $\mathrm{P}$ atom of the following base that results from the CG process. When using a structure from a previous MC simulation as the starting structure for a new MC simulation, one should use the non-minimized structure to prevent this deformation of the DNA major and minor groove. 


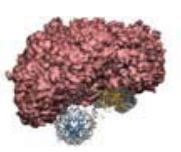

(a) $\mathrm{NCP}_{1}$ All

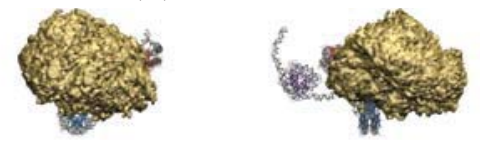

(d) $\mathrm{NCP}_{3}$ All
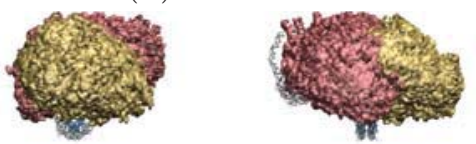

(g) $\mathrm{NCP}_{1} \& \mathrm{NCP}_{3}$ All

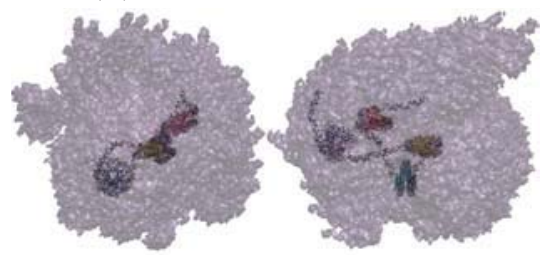

(j) $\mathrm{NCP}_{4}$ All

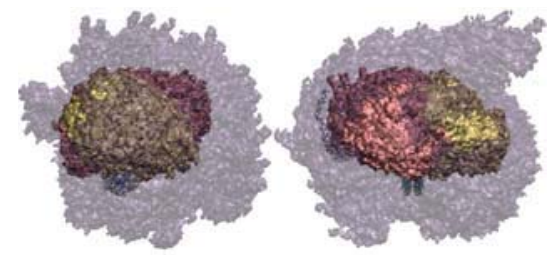

(m) All NCPs, All Structures
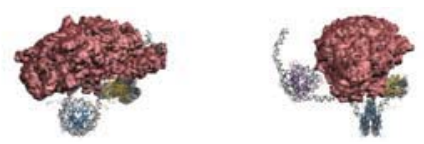

(b) $\mathrm{NCP}_{1}$ Best
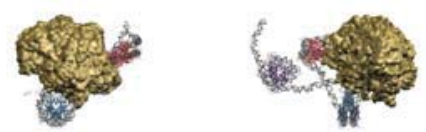

(e) $\mathrm{NCP}_{3}$ Best
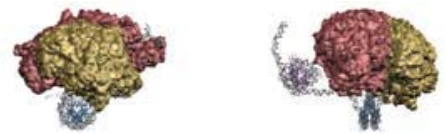

(h) $\mathrm{NCP}_{1} \& \mathrm{NCP}_{3}$ Best
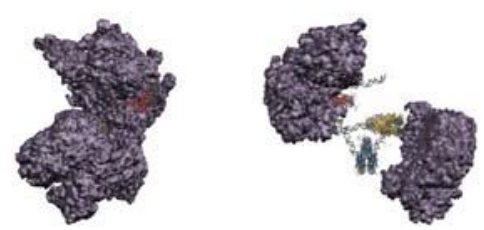

(k) $\mathrm{NCP}_{4}$ Best
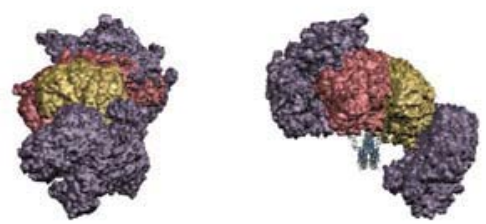

(n) All NCPs, Best Structures
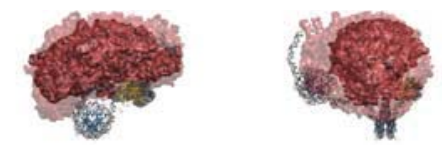

(c) $\mathrm{NCP}_{1}$ All vs Best
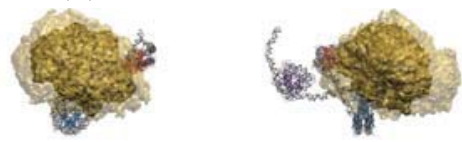

(f) $\mathrm{NCP}_{3}$ All vs Best
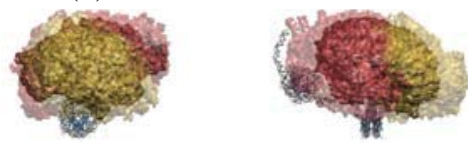

(i) $\mathrm{NCP}_{1} \& \mathrm{NCP}_{3}$ All vs Best
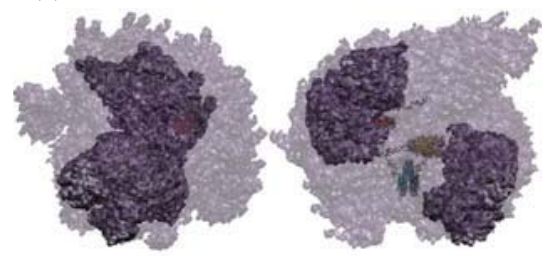

(l) $\mathrm{NCP}_{4}$ All vs Best

Figure 87. Spatial Range of the Complete vs Best Ensembles: Visualizations of the spatial range envelopes of each NCP in the nucleosome array, overlain on an illustration of an example structure from the best 500 structures. As a reference point, each structure in the ensemble was aligned using the second $\mathrm{NCP}$ in the array $\left(\mathrm{NCP}_{2}\right)$, illustrated with a blue protein core. In these illustrations, we position $\mathrm{NCP}_{2}$ closest to the bottom of the page with the NCP dyad oriented toward the top of the page. Each spatial range is shown using two views, the one on the left looking at the face of $\mathrm{NCP}_{2}$ and the one on the right looking at the side of $\mathrm{NCP}_{2}$. As there are two flexible linkers between $\mathrm{NCP}_{4}$ and the aligned $\mathrm{NCP}, \mathrm{NCP}_{2}$, the spatial range covered by $\mathrm{NCP}_{4}$ obscures the view of $\mathrm{NCP}_{1}$ and $\mathrm{NCP}_{3}$. We therefore separate the spatial ranges of $\mathrm{NCP}_{1}$ (red), $\mathrm{NCP}_{3}$ (yellow), and $\mathrm{NCP}_{4}$ (purple) as follows. The first row shows only the range of $\mathrm{NCP}_{1},(\mathrm{a})-(\mathrm{c})$. The second row shows only the range of $\mathrm{NCP}_{3}$, (d)-(f). The third row show the ranges of both $\mathrm{NCP}_{1}$ and $\mathrm{NCP}_{3}$, (g)-(i). The fourth row shows only the range of $\mathrm{NCP}_{4},(\mathrm{j})-(\mathrm{l})$. The fifth row show the combined ranges of all NCPs, (m) and (n). For all rows, the first column shows the range of the complete ensemble - (a), (d), (g), (j), and (m) - the second column shows the range of the sub-ensemble of the best 500 structures - (b), (e), (h), (k), and (n) - and the third column compares the range of the complete ensemble with the best sub-ensemble-(c), (f), (i), and (l). We exclude what would be panel (o), an overlain image of the ranges with all NCPs, because of an inability to show sufficient levels of transparency). 


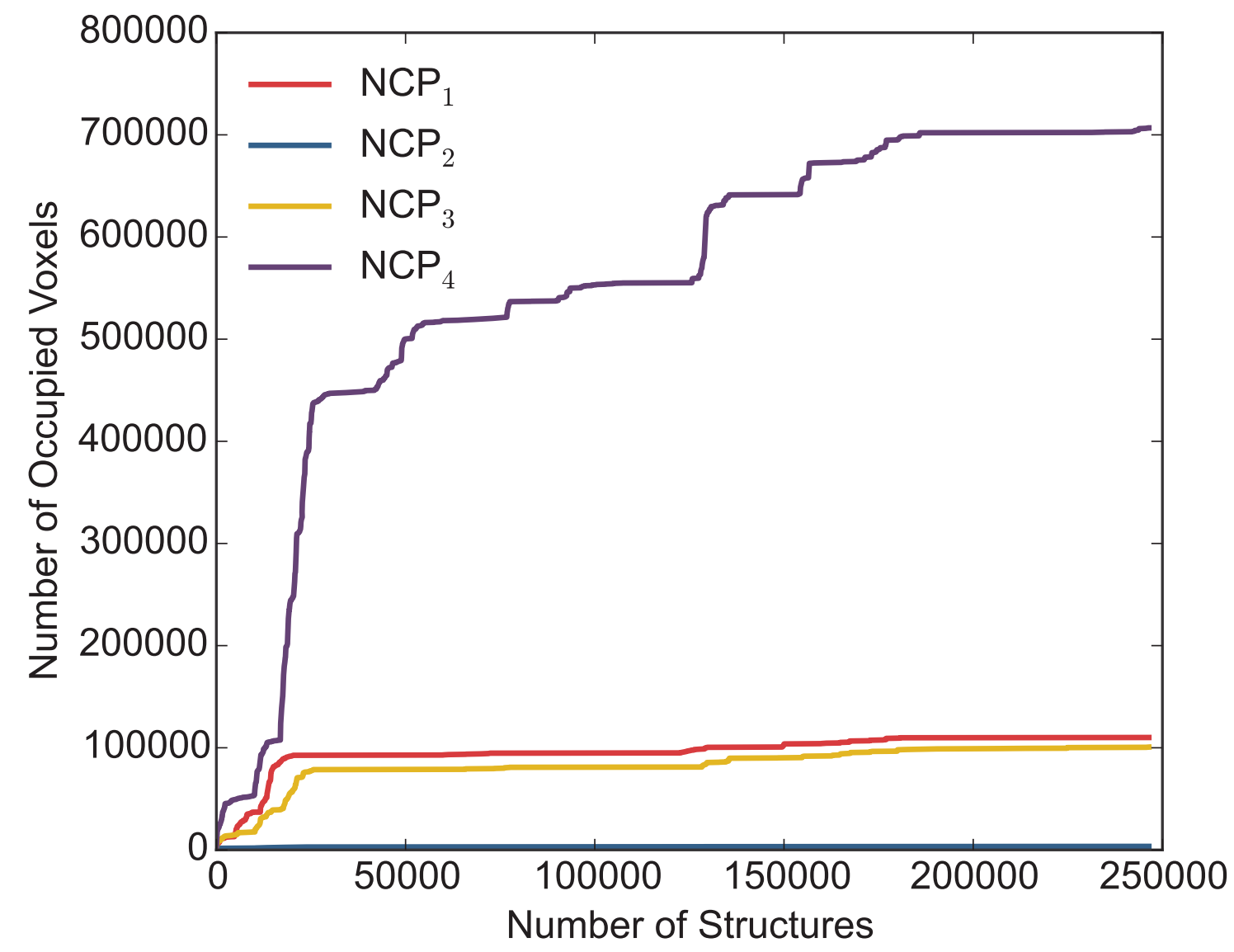

Figure 88. Convergence of Each NCP in the Tetranucleosome Array Ensemble: Separating the spatial convergence of the tetranucleosome ensemble, shown in fig. 53(c), by each of the NCPs in thewe verify good convergence for all four NCPs. This is demonstrated by the consistent number of occupied voxels as additional structures are added to the ensemble. 